Supporting Information

\title{
Iridium-Catalyzed Regioselective Hydroalkynylation of In- ternal Alkenes Directed by an Oxime
}

\author{
Peng-Chao Gao, Zi-Xuan Wang, Bi-Jie Li* \\ Center of Basic Molecular Science (CBMS), Department of Chemistry, Tsinghua University
}

\section{Table of Contents}

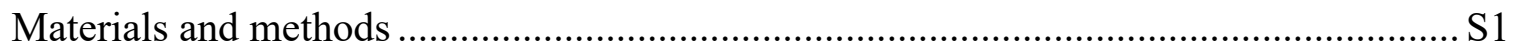

Synthesis and characterization of starting materials ............................................ S2

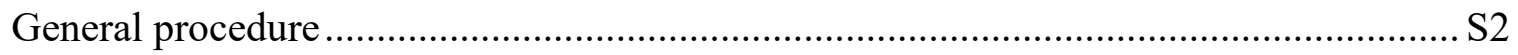

General procedure for Ir-catalyzed hydroalkynylation .............................................S13

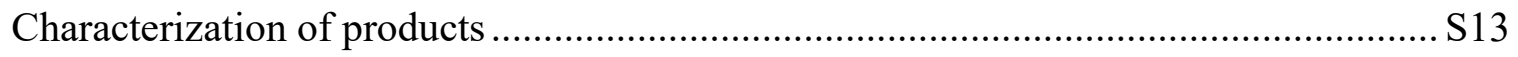

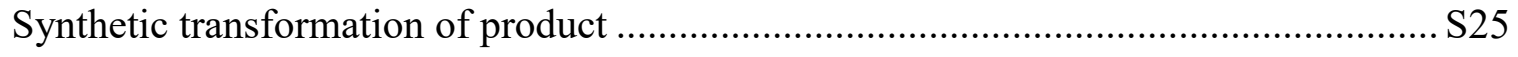

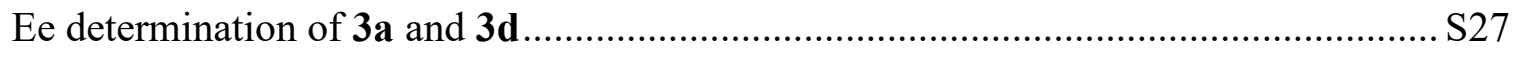

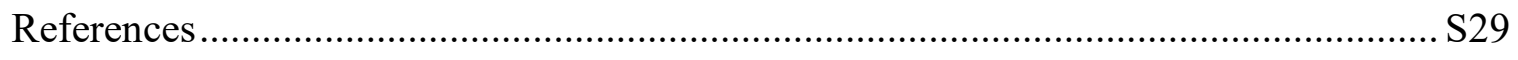

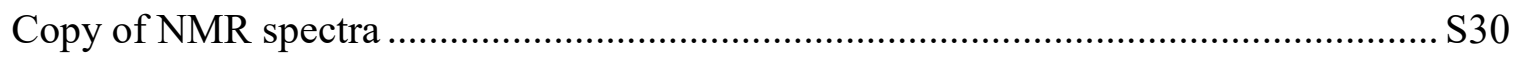




\section{Materials and methods}

Unless otherwise noted, all reactions were assembled on a Schlenk vacuum line or in an Ar filled glovebox using oven-dried glassware and were stirred with Teflon-coated magnetic stirring bars. All ligands were purchased from Strem Chemicals and were used as received. $\left[\operatorname{Ir}(\mathrm{COD})_{2} \mathrm{OTf}\right]$ was prepared according to literature methods. ${ }^{1}$ Triisopropylsilylacetylene was purchased from Alfa Aesar and was used as received. All solvents and reagents were used as received. All work-up and purification procedures were carried out with reagent grade solvents with Schlenk techniques unless otherwise specified. Reaction temperatures above $23{ }^{\circ} \mathrm{C}$ refer to temperatures of a silicon oil bath, which were controlled by an electronic temperature modulator from IKA. NMR spectra were acquired on NMR spectrometer with $400 \mathrm{MHz}$ for ${ }^{1} \mathrm{H}$ NMR and $101 \mathrm{MHz}$ for ${ }^{13} \mathrm{C}$ NMR at the NMR facility at Center of Basic Molecular Science (CBMS). Chemical shifts $(\delta)$ are reported in ppm relative to the residual solvent signal. Data for ${ }^{1} \mathrm{H}$ NMR spectra are reported as follows: chemical shift (multiplicity, coupling constants, number of hydrogens). Abbreviations are as follows: $\mathrm{s}$ (singlet), $\mathrm{d}$ (doublet), $\mathrm{t}$ (triplet), $\mathrm{q}$ (quartet), $\mathrm{m}$ (multiplet), br (broad). The HRMS analysis was carried out on UHPLC-Q-Exactive mass spectrometry system (Thermo Fisher, USA) consisting of an U3000 series ultra-high performance liquid chromatography system connected to an ion trap detector with an electrospray ionization interface in the positive ion mode. Enantiomeric excess (ee) values were determined by analytical liquid chromatography (HPLC) analysis on a Shimadzu chromatograph (Daicel chiral columns Chiralpak OD-H (4.6 x $250 \mathrm{~mm})$ ). 


\section{Synthesis and characterization of starting materials}

\section{General procedure}

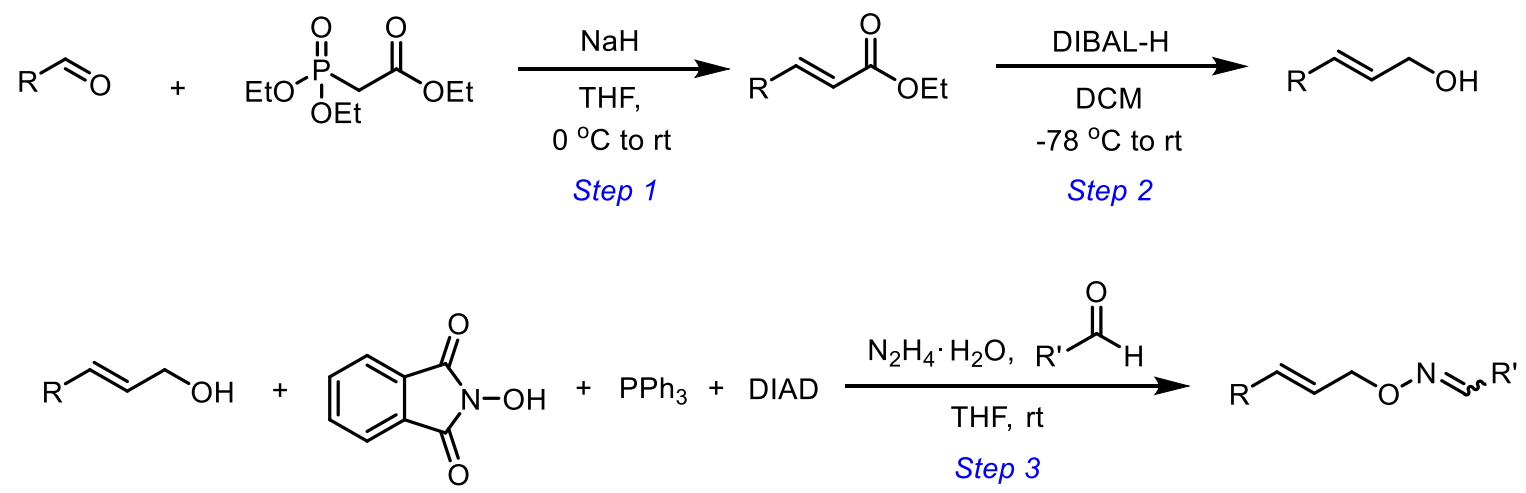

Step 1: To a solution of THF $(0.40 \mathrm{M})$ and $\mathrm{NaH}$ (60\% dispersion in mineral oil, 1.1 equiv.), triethyl phosphonoacetate ( 1.1 equiv.) was added dropwise at $0{ }^{\circ} \mathrm{C}$. The reaction was stirred for $30 \mathrm{~min}$, corresponding aldehyde (1.0 equiv.) was added slowly in THF. The reaction was stirred for another 5 minutes before quenched with saturated $\mathrm{NH}_{4} \mathrm{Cl}$. The mixture was extracted with ethyl acetate. The organic phase was collected and dried with $\mathrm{Na}_{2} \mathrm{SO}_{4}$. The solvent was evaporated, and the residue was purified by silica gel column chromatography with EtOAc/Hexane as eluent to give the $\alpha, \beta$-unsaturated ester. All the yields are not optimized.

Step 2: To a solution of $\alpha, \beta$-unsaturated ester (1.0 equiv.) in dry DCM $(0.50 \mathrm{M})$ was added DIBAL-H (2.1 equiv.) dropwise at $-78^{\circ} \mathrm{C}$. The cooling bath was removed and the reaction temperature was slowly warmed to room temperature. The mixture was stirred for $2 \mathrm{~h}$ and quenched with water (4.7 equiv.), $15 \% \mathrm{NaOH}$ (9.4 equiv.) and water (4.7 equiv.) sequentially at $0{ }^{\circ} \mathrm{C}$. The mixture was stirred for another $15 \mathrm{~min}$ and filtered through Celite. The solvent was evaporated and the residue was purified by silica gel column chromatography with EtOAc/Hexane as eluent to give the allylic alcohol. All the yields are not optimized.

Step 3: A Schlenk flask was charged with THF (0.20 M), 2-hydroxyisoindoline-1,3-dione (1.1 equiv.) and $\mathrm{PPh}_{3}$ (1.1 equiv.) were added under Ar. Allylic alcohol (1.0 equiv.) was added, followed by DIAD (1.1 equiv.). The reaction was stirred for $30 \mathrm{~min}$ and hydrazine hydrate (2.0 equiv.) was added. The mixture was stirred for $15 \mathrm{~min}$ and the corresponding 
aldehyde (2.0 equiv.) was added. The mixture was stirred overnight. The solvent was removed under reduced pressure. The residue was purified by column chromatography with EtOAc/Hexane as eluent to give the desired oxime product. All the yields are not optimized.

\section{Characterization of starting materials}

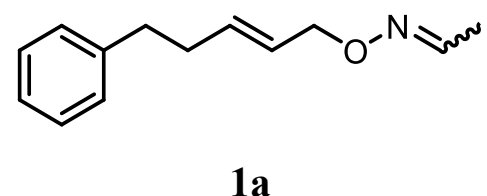

The titled compound was prepared according to General Procedure in $6.2 \mathrm{mmol}$ scale. Colorless oil. $819 \mathrm{mg}, 65 \%$ Yield. Spectroscopic data are given as a mixture of cis and trans isomers (1:1). ${ }^{1} \mathbf{H}$ NMR (400 MHz, Chloroform- $d$ ) $\delta 7.44(\mathrm{q}, J=5.9 \mathrm{~Hz}, 0.5 \mathrm{H}), 7.30$ $(\mathrm{m}, 2 \mathrm{H}), 7.19(\mathrm{~m}, 3 \mathrm{H}), 6.77(\mathrm{q}, J=5.5 \mathrm{~Hz}, 0.5 \mathrm{H}), 5.77(\mathrm{~m}, 1 \mathrm{H}), 5.70(\mathrm{~m}, 1 \mathrm{H}), 4.50(\mathrm{~m}$, 2H), $2.72(\mathrm{~m}, 2 \mathrm{H}), 2.40$ (m, 2H), 1.85 (m, 3H). ${ }^{13} \mathbf{C ~ N M R}(101 \mathrm{MHz}$, Chloroform-d) $\delta 147.0$, $141.9,141.9,134.6,134.1,128.6,128.6,128.4,126.7,126.3,126.0,74.5,74.2,35.6,35.6$, 34.3, 34.3, 15.4, 12.1. IR $v\left(\mathrm{~cm}^{-1}\right)$ 2921, 2361, 2341, 1020, 970, 669. HRMS (ESI-ion trap) calcd for $\mathrm{C}_{13} \mathrm{H}_{18} \mathrm{NO}^{+}\left([\mathrm{M}+\mathrm{H}]^{+}\right)$204.1383, found 204.1378

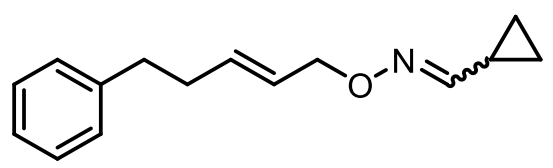

1b

The titled compound was prepared according to General Procedure in $2.0 \mathrm{mmol}$ scale. Colorless oil. $252 \mathrm{mg}, 55 \%$ Yield. Spectroscopic data are given as a mixture of cis and trans isomers (1:1). ${ }^{1} \mathbf{H}$ NMR (400 MHz, Chloroform- $\left.d\right) \delta 7.28(\mathrm{~m}, 2 \mathrm{H}), 7.20(\mathrm{~m}, 3 \mathrm{H}), 6.90$ $(\mathrm{d}, J=8.5 \mathrm{~Hz}, 0.5 \mathrm{H}), 5.95(\mathrm{~d}, J=8.9 \mathrm{~Hz}, 0.5 \mathrm{H}), 5.81(\mathrm{~m}, 1 \mathrm{H}), 5.71(\mathrm{~m}, 1 \mathrm{H}), 4.50(\mathrm{~m}, 2 \mathrm{H})$, $2.72(\mathrm{~m}, 2 \mathrm{H}), 2.40(\mathrm{~m}, 2 \mathrm{H}), 2.23(\mathrm{~m}, 0.5 \mathrm{H}), 1.63(\mathrm{~m}, 0.6 \mathrm{H}), 0.90(\mathrm{~m}, 2 \mathrm{H}), 0.61(\mathrm{~m}, 2 \mathrm{H}) .{ }^{13} \mathrm{C}$ NMR (101 MHz, Chloroform-d) $\delta 154.9,154.4,141.9,141.9,134.6,134.1,128.5,128.5$, $128.4,126.7,126.3,126.0,125.9,74.6,74.3,35.6,35.6,34.3,11.1,8.0,6.3,5.7$ IR $v\left(\mathrm{~cm}^{-}\right.$ 1) $2923,2857,1732,1671,970,699$. HRMS (ESI-ion trap) calcd for $\mathrm{C}_{15} \mathrm{H}_{20} \mathrm{NO}^{+}\left([\mathrm{M}+\mathrm{H}]^{+}\right)$ 230.1539 , found 230.1532 


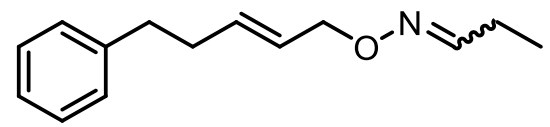

$1 \mathrm{c}$

The titled compound was prepared according to General Procedure in 2.4 mmol scale. Colorless oil. $328 \mathrm{mg}, 63 \%$ Yield. Spectroscopic data are given as a mixture of cis and trans isomers (1.2:1). ${ }^{1} \mathbf{H}$ NMR (400 MHz, Chloroform- $\left.d\right) \delta 7.41(\mathrm{t}, J=5.9 \mathrm{~Hz}, 0.4 \mathrm{H})$, $7.27(\mathrm{~m}, 2 \mathrm{H}), 7.19(\mathrm{~m}, 3 \mathrm{H}), 6.63(\mathrm{t}, J=5.3 \mathrm{~Hz}, 1 \mathrm{H}), 5.78(\mathrm{~m}, 1 \mathrm{H}), 5.70(\mathrm{~m}, 1 \mathrm{H}), 4.49(\mathrm{~m}$, 2H), $2.72(\mathrm{~m}, 2 \mathrm{H}), 2.36(\mathrm{~m}, 3 \mathrm{H}), 2.22(\mathrm{~m}, 1 \mathrm{H}), 1.08$ (m, 3H). ${ }^{13} \mathbf{C}$ NMR (101 MHz, Chloroform-d) $\delta 153.4,152.2,141.9,134.7,134.0,128.6,128.6,128.5,128.4,126.7,126.2$, 126.0, 126.0, 74.6, 74.3, 35.6, 35.6, 34.3, 34.3, 23.2, 19.3, 11.3, 10.8. IR v (cm $\left.{ }^{-1}\right) 3027$, 2971, 2922, 1496, 1454, 970, 699. HRMS (ESI-ion trap) calcd for $\mathrm{C}_{14} \mathrm{H}_{20} \mathrm{NO}^{+}\left([\mathrm{M}+\mathrm{H}]^{+}\right)$ 218.1539 , found 218.1532

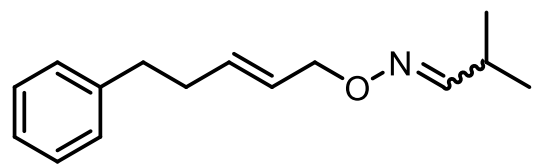

1d

The titled compound was prepared according to General Procedure in $1.2 \mathrm{mmol}$ scale. Colorless oil. $203 \mathrm{mg}, 72 \%$ Yield. Spectroscopic data are given as a mixture of cis and trans isomers (4:1). ${ }^{1} \mathbf{H}$ NMR (400 MHz, Chloroform-d) $\delta 7.29(\mathrm{~m}, 2 \mathrm{H}), 7.19(\mathrm{~m}, 3 \mathrm{H}), 6.48$ $(\mathrm{d}, J=7.2 \mathrm{~Hz}, 0.2 \mathrm{H}), 5.79(\mathrm{~m}, 1 \mathrm{H}), 5.68(\mathrm{~m}, 1 \mathrm{H}), 4.48(\mathrm{~m}, 2 \mathrm{H}), 3.13(\mathrm{~m}, 0.2 \mathrm{H}), 2.72(\mathrm{~m}$, 2H), $2.50(\mathrm{~m}, 0.8 \mathrm{H}), 2.39(\mathrm{~m}, 2 \mathrm{H}), 1.06(\mathrm{~m}, 6 \mathrm{H}) .{ }^{13} \mathrm{C}$ NMR (101 MHz, Chloroform- $d$ ) $\delta 157.5,156.1,141.9,134.7,133.8,128.6,128.5,128.5,128.4,126.8,126.2,126.0,126.0$, 74.5, 74.2, 35.6, 35.6, 34.3, 34.3, 29.5, 25.2, 20.3, 19.9. IR $v\left(\mathrm{~cm}^{-1}\right) 2959,2865,2361$, 2341, 2164, 1496, 1060, 699. HRMS (ESI-ion trap) calcd for $\mathrm{C}_{15} \mathrm{H}_{22} \mathrm{NO}^{+}\left([\mathrm{M}+\mathrm{H}]^{+}\right)$ 232.1696, found 232.1689

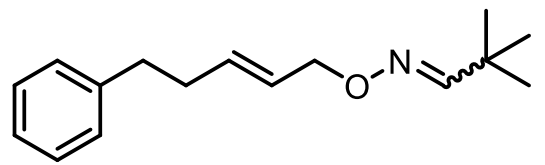

1e 
The titled compound was prepared according to General Procedure in $2.0 \mathrm{mmol}$ scale. Colorless oil. $289 \mathrm{mg}, 59 \%$ Yield. Spectroscopic data are given as a mixture of cis and trans isomers (10:1). ${ }^{1} \mathbf{H}$ NMR (400 MHz, Chloroform- $\left.d\right) \delta 7.29(\mathrm{~m}, 3 \mathrm{H}), 7.20(\mathrm{dd}, J=6.5$, $3.5 \mathrm{~Hz}, 3 \mathrm{H}), 5.79(\mathrm{~m}, 1 \mathrm{H}), 5.71(\mathrm{~m}, 1 \mathrm{H}), 4.47$ (m, 2H), 2.72 (dd, $J=9.3,6.5 \mathrm{~Hz}, 2 \mathrm{H}), 2.39$ $(\mathrm{d}, J=7.8 \mathrm{~Hz}, 2 \mathrm{H}), 1.10(\mathrm{~s}, 9 \mathrm{H}) .{ }^{13} \mathbf{C}$ NMR (101 MHz, Chloroform- $d$ ) $\delta$ 158.5, 141.9, $134.7,128.5,128.5,128.5,126.2,126.0,74.3,35.6,34.3,33.7,27.8$. IR $v\left(\mathrm{~cm}^{-1}\right) 2962$, 2929, 2865, 2360, 2341, 1365, 1023, 669. HRMS (ESI-ion trap) calcd for $\mathrm{C}_{16} \mathrm{H}_{24} \mathrm{NO}^{+}$ $\left([\mathrm{M}+\mathrm{H}]^{+}\right) 246.1852$, found 246.1845

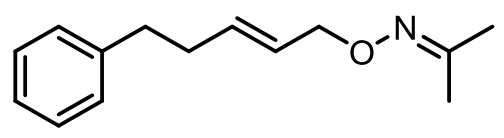

1f

The titled compound was prepared according to General Procedure in $10 \mathrm{mmol}$ scale. Colorless oil. 1.4 g, 64\% Yield. ${ }^{1}$ H NMR (400 MHz, Chloroform- $d$ ) $\delta 7.28$ (m, 2H), 7.19 (m, 3H), 5.77 (m, 1H), $5.70(\mathrm{~m}, 1 \mathrm{H}), 4.48$ (d, J=5.9 Hz, 2H), $2.72(\mathrm{~m}, 2 \mathrm{H}), 2.39(\mathrm{~m}, 2 \mathrm{H})$, 1.87 (m, 6H). ${ }^{13}$ C NMR (101 MHz, Chloroform- $d$ ) 154.9, 142.0, 133.7, 128.6, 128.4, 126.8, 125.9, 74.1, 35.6, 34.3, 22.0, 15.8. IR $v\left(\mathrm{~cm}^{-1}\right)$ 3062, 3026, 2919, 2855, 1724, 1672, 911, 658. HRMS (ESI-ion trap) calcd for $\mathrm{C}_{14} \mathrm{H}_{20} \mathrm{NO}^{+}\left([\mathrm{M}+\mathrm{H}]^{+}\right) 218.1539$, found 218.1533

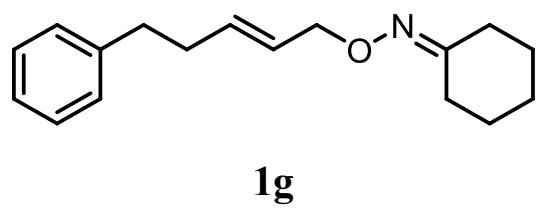

The titled compound was prepared according to General Procedure in 0.9 mmol scale. Colorless oil. $159 \mathrm{mg}, 69 \%$ Yield. ${ }^{1} \mathbf{H}$ NMR (400 MHz, Chloroform- $d$ ) $\delta 7.28$ (m, 2H), $7.19(\mathrm{~m}, 3 \mathrm{H}), 5.77(\mathrm{~m}, 1 \mathrm{H}), 5.70(\mathrm{~m}, 1 \mathrm{H}), 4.47$ (d, J=5.9 Hz, 2H), 2.72 (m, 2H), 2.47 (m, 2H), 2.39 (m, 2H), $2.21(\mathrm{~m}, 2 \mathrm{H}), 1.61(\mathrm{~m}, 6 \mathrm{H}) .{ }^{13} \mathbf{C}$ NMR (101 MHz, Chloroform-d) $\delta 160.5$, $142.0,133.8,128.6,128.4,126.7,125.9,74.0,35.6,34.3,32.4,27.2,26.0,25.9$, 25.5. IR $v\left(\mathrm{~cm}^{-1}\right)$ 2929, 2856, 2360, 2341, 1274, 970, 934. HRMS (ESI-ion trap) calcd for $\mathrm{C}_{17} \mathrm{H}_{24} \mathrm{NO}^{+}\left([\mathrm{M}+\mathrm{H}]^{+}\right) 258.1852$, found 258.1842 


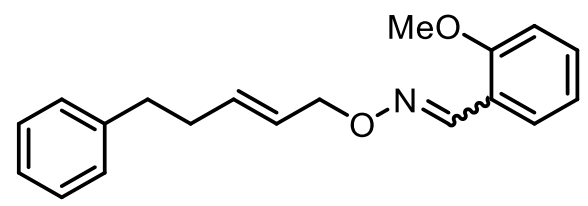

$1 \mathrm{~h}$

The titled compound was prepared according to General Procedure in $3.0 \mathrm{mmol} \mathrm{scale}$. Colorless oil. $514 \mathrm{mg}, 58 \%$ Yield. Spectroscopic data are given as a mixture of cis and trans isomers (6:1). ${ }^{1} \mathbf{H}$ NMR (400 MHz, Chloroform- $d$ ) $\delta 8.51(\mathrm{~s}, 0.86 \mathrm{H}), 7.80(\mathrm{~m}, 1 \mathrm{H})$, $7.34(\mathrm{~m}, 1 \mathrm{H}), 7.28(\mathrm{~m}, 2 \mathrm{H}), 7.20(\mathrm{~m}, 3 \mathrm{H}), 6.95(\mathrm{~m}, 1 \mathrm{H}), 6.89 \mathrm{~m}, 1 \mathrm{H}), 5.85(\mathrm{~m}, 1 \mathrm{H}), 5.78$ (m, 1H), $4.63(\mathrm{~d}, J=6.1 \mathrm{~Hz}, 2 \mathrm{H}), 3.84(\mathrm{~s}, 3 \mathrm{H}), 2.74(\mathrm{~m}, 2 \mathrm{H}), 2.42(\mathrm{~m}, 2 \mathrm{H}) .{ }^{13} \mathbf{C}$ NMR $(101$ MHz, Chloroform- $d$ ) $\delta$ 157.7, 145.0, 141.9, 134.8, 131.2, 128.6, 128.5, 128.4, 126.6, 126.3, 126.0, 121.1, 120.9, 111.2, 75.0, 55.7, 35.6, 34.4. IR $v\left(\mathrm{~cm}^{-1}\right)$ 2921, 2838, 2361, 2341, 1605, 1196, 1023, 779. HRMS (ESI-ion trap) calcd for $\mathrm{C}_{19} \mathrm{H}_{22} \mathrm{NO}_{2}{ }^{+}\left([\mathrm{M}+\mathrm{H}]^{+}\right) 296.1645$, found 296.1635

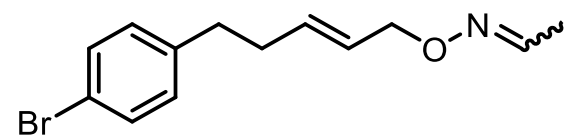

1i

The titled compound was prepared according to General Procedure in $5.5 \mathrm{mmol}$ scale. Colorless oil. $857 \mathrm{mg}, 61 \%$ Yield. Spectroscopic data are given as a mixture of cis and trans isomers (1:1). ${ }^{1} \mathbf{H}$ NMR (400 MHz, Chloroform- $d$ ) $\delta 7.43(\mathrm{~m}, 0.5 \mathrm{H}), 7.39(\mathrm{~m}, 2 \mathrm{H})$, $7.05(\mathrm{~m}, 2 \mathrm{H}), 6.76(\mathrm{q}, J=5.5 \mathrm{~Hz}, 0.5 \mathrm{H}), 5.79(\mathrm{~m}, 1 \mathrm{H}), 5.65(\mathrm{~m}, 1 \mathrm{H}), 4.48(\mathrm{~m}, 2 \mathrm{H}), 2.67$ (m, 2H), $2.35(\mathrm{~m} \mathrm{2H}), 1.85$ (m, 3H). ${ }^{13} \mathbf{C}$ NMR (101 MHz, Chloroform- $d$ ) $\delta$ 147.0, 140.8, 140.8, 133.9, 133.4, 131.5, 131.5, 130.4, 130.3, 127.1, 126.7, 119.7, 74.4, 74.1, 34.9, 34.0, 34.0, 15.4, 12.1. IR $v\left(\mathrm{~cm}^{-1}\right)$ 2920, 2857, 2361, 2341, 1488, 1010, 814. HRMS (ESI-ion trap) calcd for $\mathrm{C}_{13} \mathrm{H}_{17} \mathrm{BrNO}^{+}\left([\mathrm{M}+\mathrm{H}]^{+}\right) 282.0488$, found 282.0482

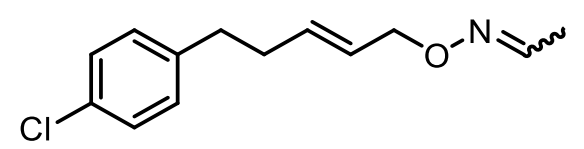

$\mathbf{1 j}$ 
The titled compound was prepared according to General Procedure in 5.0 mmol scale. Colorless oil. $830 \mathrm{mg}, 70 \%$ Yield. Spectroscopic data are given as a mixture of cis and trans isomers (1:1). ${ }^{1} \mathbf{H}$ NMR (400 MHz, Chloroform- $d$ ) $\delta 7.43$ (q, $\left.J=5.9 \mathrm{~Hz}, 0.5 \mathrm{H}\right), 7.24$ $(\mathrm{m}, 2 \mathrm{H}), 7.10(\mathrm{~m}, 2 \mathrm{H}), 6.77(\mathrm{q}, J=5.6 \mathrm{~Hz}, 0.5 \mathrm{H}), 5.74(\mathrm{~m}, 1 \mathrm{H}), 5.67(\mathrm{~m}, 1 \mathrm{H}), 4.48(\mathrm{~m}$, 2H), $2.68(\mathrm{~m}, 2 \mathrm{H}), 2.36(\mathrm{~m}, 2 \mathrm{H}), 1.85$ (m, 3H). ${ }^{13} \mathbf{C}$ NMR (101 MHz, Chloroform- $d$ ) $\delta 147.1$, $140.3,140.3,134.0,133.5,131.7,129.9,129.9,128.5,128.5,127.1,126.7,74.4,74.1,72.8$, 72.5, 34.9, 34.1, 34.1, 31.7, 31.6, 30.8, 30.7, 15.4, 12.1. IR $v\left(\mathrm{~cm}^{-1}\right) 2920,2858,2361$, 2341, 1492, 1091, 1014, 818. HRMS (ESI-ion trap) calcd for $\mathrm{C}_{13} \mathrm{H}_{17} \mathrm{ClNO}^{+}\left([\mathrm{M}+\mathrm{H}]^{+}\right)$ 238.0993, found 238.0984

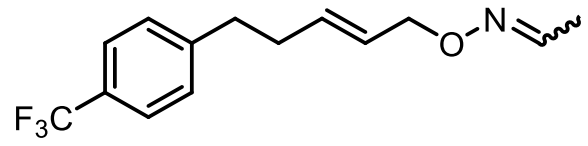

$1 \mathbf{k}$

The titled compound was prepared according to General Procedure in $4.0 \mathrm{mmol}$ scale. Colorless oil. $846 \mathrm{mg}, 78 \%$ Yield. Spectroscopic data are given as a mixture of cis and trans isomers (1:1). ${ }^{1} \mathbf{H}$ NMR (400 MHz, Chloroform-d) $\delta 7.54(\mathrm{~m}, 2.5 \mathrm{H}), 7.28(\mathrm{~m}, 2 \mathrm{H})$, $6.76(\mathrm{q}, J=5.3 \mathrm{~Hz}, 0.5 \mathrm{H}), 5.75(\mathrm{~m}, 1 \mathrm{H}), 5.69(\mathrm{~m}, 1 \mathrm{H}), 4.49(\mathrm{~m}, 2 \mathrm{H}), 2.77(\mathrm{~m}, 2 \mathrm{H}), 2.40$ $(\mathrm{m}, 2 \mathrm{H}), 1.84(\mathrm{t}, J=5.7 \mathrm{~Hz}, 3 \mathrm{H}) .{ }^{13} \mathrm{C}$ NMR (101 MHz, Chloroform- $d$ ) $\delta 147.1,145.9$, $133.7,133.5,133.2,128.9,128.9,127.3,127.1,127.0,125.7,125.4,125.3,125.3,74.3$, 74.1, 35.4, 35.3, 33.9, 33.9, 15.4, 12.1. IR $\vee\left(\mathrm{cm}^{-1}\right)$ 2923, 2859, 2360, 2342, 1619, 1324, 1119, 1067, 1018, 827. HRMS (ESI-ion trap) calcd for $\mathrm{C}_{14} \mathrm{H}_{17} \mathrm{~F}_{3} \mathrm{NO}^{+}\left([\mathrm{M}+\mathrm{H}]^{+}\right) 272.1257$, found 272.1253

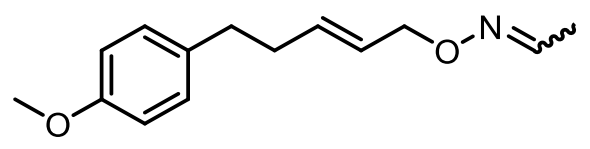

11

The titled compound was prepared according to General Procedure in $5.0 \mathrm{mmol}$ scale. Colorless oil. $944 \mathrm{mg}, 81 \%$ Yield. Spectroscopic data are given as a mixture of cis and trans isomers (1:1). ${ }^{1} \mathbf{H}$ NMR (400 MHz, Chloroform- $d$ ) $\delta 7.43(\mathrm{q}, J=5.9 \mathrm{~Hz}, 0.5 \mathrm{H}), 7.09$ $(\mathrm{m}, 2 \mathrm{H}), 6.83(\mathrm{~m}, 2 \mathrm{H}), 6.77(\mathrm{~m}, 0.5 \mathrm{H}), 5.77(\mathrm{~m}, 1 \mathrm{H}), 5.69(\mathrm{~m}, 1 \mathrm{H}), 4.52(\mathrm{~m}, 2 \mathrm{H}), 3.79(\mathrm{~s}$, 3H), $2.66(\mathrm{~m}, 2 \mathrm{H}), 2.35(\mathrm{~m}, 2 \mathrm{H}), 1.85(\mathrm{~m}, 3 \mathrm{H}) .{ }^{13} \mathrm{C}$ NMR (101 MHz, Chloroform-d) $\delta$ 
$157.9,147.0,134.7,134.2,134.0,129.4,126.6,126.2,113.9,74.6,74.3,55.4,34.7,34.6$, 34.6, 15.4, 12.1. IR $v\left(\mathrm{~cm}^{-1}\right)$ 2919, 2361, 2341, 1511, 1243, 1020, 824. HRMS (ESI-ion trap) calcd for $\mathrm{C}_{14} \mathrm{H}_{20} \mathrm{NO}_{2}{ }^{+}\left([\mathrm{M}+\mathrm{H}]^{+}\right) 234.1489$, found 234.1479

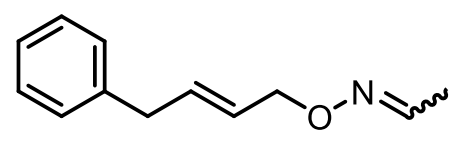

$1 \mathrm{~m}$

The titled compound was prepared according to General Procedure in $6.0 \mathrm{mmol}$ scale. Colorless oil. $760 \mathrm{mg}, 67 \%$ Yield. Spectroscopic data are given as a mixture of cis and trans isomers (1:1). ${ }^{1} \mathbf{H}$ NMR (400 MHz, Chloroform- $d$ ) $\delta 7.44$ (q, $\left.J=5.8 \mathrm{~Hz}, 0.5 \mathrm{H}\right), 7.30$ $(\mathrm{m}, 2 \mathrm{H}), 7.20(\mathrm{~m}, 3 \mathrm{H}), 6.78(\mathrm{~m}, 0.5 \mathrm{H}), 5.90(\mathrm{~m}, 1 \mathrm{H}), 5.74(\mathrm{~m}, 1 \mathrm{H}), 4.54(\mathrm{~m}, 2 \mathrm{H}), 3.42(\mathrm{~m}$, 2H), $1.85(\mathrm{~m}, 3 \mathrm{H}) .{ }^{13} \mathbf{C}$ NMR (101 MHz, Chloroform- $d$ ) $\delta$ 147.0, 140.1, 140.0, 133.7, 133.2, $131.9,128.7,128.6,128.5,128.3,127.6,127.2,127.2,126.7,126.2,126.2,74.3,74.0,73.1$, 72.8, 38.9, 33.1, 33.0, 15.3, 12.1. IR $v\left(\mathrm{~cm}^{-1}\right)$ 3027, 2921, 2360, 1495, 1020, 699 HRMS (ESI-ion trap) calcd for $\mathrm{C}_{12} \mathrm{H}_{16} \mathrm{NO}^{+}\left([\mathrm{M}+\mathrm{H}]^{+}\right)$190.1226, found 190.1221

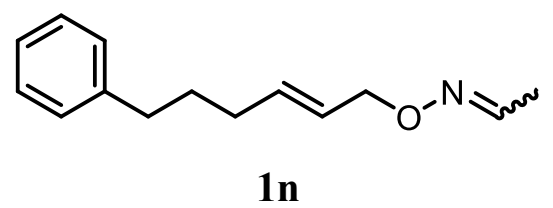

The titled compound was prepared according to General Procedure in $5.0 \mathrm{mmol} \mathrm{scale}$. Colorless oil. $771 \mathrm{mg}, 71 \%$ Yield. Spectroscopic data are given as a mixture of cis and trans isomers (1:1). ${ }^{1} \mathbf{H}$ NMR (400 MHz, Chloroform- $d$ ) $\delta 7.44$ (q, $\left.J=5.9 \mathrm{~Hz}, 0.5 \mathrm{H}\right), 7.29$ (m, 2H), $7.19(\mathrm{~m}, 3 \mathrm{H}), 6.77$ (q, $J=5.6 \mathrm{~Hz}, 0.5 \mathrm{H}), 5.79(\mathrm{~m}, 1 \mathrm{H}), 5.68(\mathrm{~m}, 1 \mathrm{H}), 4.51(\mathrm{~m}$, 2H), $2.63(\mathrm{~m}, 2 \mathrm{H}), 2.12(\mathrm{~m}, 2 \mathrm{H}), 1.85(\mathrm{~m}, 3 \mathrm{H}), 1.74(\mathrm{~m}, 2 \mathrm{H}) .{ }^{13} \mathbf{C}$ NMR (101 MHz, Chloroform-d) $\delta 147.0,147.0,142.5,135.2,134.7,128.6,128.5,128.4,126.5,126.1,125.8$, 74.6, 74.3, 35.5, 32.0, 30.8, 30.8, 15.4, 12.1. IR $v\left(\mathrm{~cm}^{-1}\right)$ 2922, 2857, 2361, 2341, 1454, 1016, 968, 670. HRMS (ESI-ion trap) calcd for $\mathrm{C}_{14} \mathrm{H}_{20} \mathrm{NO}^{+}\left([\mathrm{M}+\mathrm{H}]^{+}\right) 218.1539$, found 218.1534

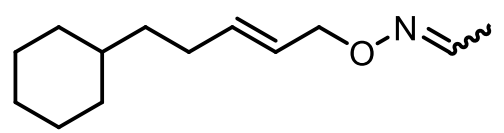

10 
The titled compound was prepared according to General Procedure in 6.0 mmol scale. Colorless oil. $891 \mathrm{mg}, 71 \%$ Yield. Spectroscopic data are given as a mixture of cis and trans isomers (1:1). ${ }^{1} \mathbf{H}$ NMR (400 MHz, Chloroform- $d$ ) $\delta 7.42$ (q, $\left.J=5.7 \mathrm{~Hz}, 0.5 \mathrm{H}\right), 6.76$ $(\mathrm{q}, J=5.5 \mathrm{~Hz}, 0.5 \mathrm{H}), 5.73(\mathrm{~m}, 1 \mathrm{H}), 5.62(\mathrm{~m}, 1 \mathrm{H}), 4.50(\mathrm{~m}, 2 \mathrm{H}), 2.06(\mathrm{~m}, 2 \mathrm{H}), 1.84(\mathrm{~m}$, 3H), $1.65(\mathrm{~m}, 6 \mathrm{H}), 1.22(\mathrm{~m}, 7 \mathrm{H}), 0.87(\mathrm{~m}, 2 \mathrm{H}) .{ }^{13} \mathbf{C}$ NMR (101 MHz, Chloroform- $d$ ) $\delta 146.9$, 136.3, 135.7, 125.7, 125.3, 74.8, 74.5, 74.4, 74.1, 37.6, 37.3, 37.3, 36.8, 36.8, 33.7, 33.7, $33.5,33.5,33.4,29.8,26.8,26.6,26.5,26.5,15.4,12.1,12.0 . \mathbf{I R} v\left(\mathrm{~cm}^{-1}\right) 2920,2850,2361$, 2341, 1448, 1022, 971. HRMS (ESI-ion trap) calcd for $\mathrm{C}_{13} \mathrm{H}_{24} \mathrm{NO}^{+}\left([\mathrm{M}+\mathrm{H}]^{+}\right)$210.1852, found 210.1849

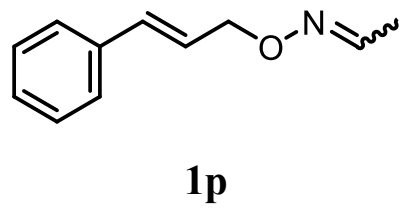

The titled compound was prepared according to General Procedure in $5.0 \mathrm{mmol}$ scale. Colorless oil. $657 \mathrm{mg}, 75 \%$ Yield. Spectroscopic data are given as a mixture of cis and trans isomers (1:1). ${ }^{1} \mathbf{H}$ NMR (400 MHz, Chloroform- $d$ ) $\delta 7.48$ (q, $\left.J=5.8 \mathrm{~Hz}, 0.5 \mathrm{H}\right), 7.40$ $(\mathrm{m}, 2 \mathrm{H}), 7.32(\mathrm{~m}, 2 \mathrm{H}), 7.23(\mathrm{~m}, 1 \mathrm{H}), 6.81(\mathrm{q}, J=5.5 \mathrm{~Hz}, 0.5 \mathrm{H}), 6.64(\mathrm{~m}, 1 \mathrm{H}), 6.37(\mathrm{~m}$, 1H), 4.72 (m, 2H), 1.88 (m, 3H). ${ }^{13}$ C NMR (101 MHz, Chloroform-d) $\delta$ 147.3, 136.8, $133.3,133.0,128.7,127.9,127.9,126.7,125.8,125.5,74.5,74.2,15.4,12.1$. IR $v\left(\mathrm{~cm}^{-1}\right)$ 2919, 2855, 2361, 2341, 1436, 1017, 747, 692. HRMS (ESI-ion trap) calcd for $\mathrm{C}_{11} \mathrm{H}_{14} \mathrm{NO}^{+}$ $\left([\mathrm{M}+\mathrm{H}]^{+}\right) 176.1070$, found 176.1068

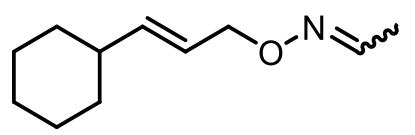

$1 \mathbf{q}$

The titled compound was prepared according to General Procedure in $5.0 \mathrm{mmol}$ scale. Colorless oil. $661 \mathrm{mg}, 73 \%$ Yield. Spectroscopic data are given as a mixture of cis and trans isomers (1:1). ${ }^{1} \mathbf{H}$ NMR (400 MHz, Chloroform- $d$ ) $\delta 7.43$ (q, $\left.J=5.9 \mathrm{~Hz}, 0.5 \mathrm{H}\right), 6.78$ $(\mathrm{q}, J=5.5 \mathrm{~Hz}, 0.5 \mathrm{H}), 5.70(\mathrm{~m}, 1 \mathrm{H}), 5.62(\mathrm{~m}, 1 \mathrm{H}), 4.51(\mathrm{~m}, 2 \mathrm{H}), 2.01(\mathrm{~m}, 1 \mathrm{H}), 1.87(\mathrm{~m}$, 3H), $1.65(\mathrm{~m}, 6 \mathrm{H}), 1.21(\mathrm{~m}, 5 \mathrm{H}) .{ }^{13} \mathbf{C}$ NMR (101 MHz, Chloroform- $d$ ) $\delta 146.9,146.9$, $141.5,140.9,123.4,122.9,75.0,74.7,40.5,32.8,32.8,26.3,26.2,15.4,12.1$. IR $v\left(\mathrm{~cm}^{-1}\right)$ 
2922, 2851, 2360, 2341, 1448, 1022, 970. HRMS (ESI-ion trap) calcd for $\mathrm{C}_{11} \mathrm{H}_{20} \mathrm{NO}^{+}$ $\left([\mathrm{M}+\mathrm{H}]^{+}\right) 182.1539$, found 182.1537

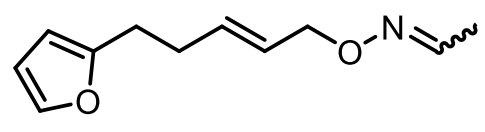

$1 \mathbf{r}$

The titled compound was prepared according to General Procedure in $6.0 \mathrm{mmol}$ scale. Colorless oil. $869 \mathrm{mg}, 75 \%$ Yield. Spectroscopic data are given as a mixture of cis and trans isomers (1:1). ${ }^{1} \mathbf{H}$ NMR (400 MHz, Chloroform- $d$ ) $\delta 7.43$ (q, $\left.J=5.9 \mathrm{~Hz}, 0.5 \mathrm{H}\right), 7.30$ (m, 1H), 6.77 (q, $J=5.5 \mathrm{~Hz}, 0.5 \mathrm{H}), 6.27(\mathrm{~m}, 1 \mathrm{H}), 5.99(\mathrm{~m}, 1 \mathrm{H}), 5.76(\mathrm{~m}, 1 \mathrm{H}), 5.71(\mathrm{~m}$, 1H), 4.50 (m, 2H), $2.73(\mathrm{~m}, 2 \mathrm{H}), 2.42$ (m, 2H), 1.85 (m, 3H). ${ }^{13}$ C NMR (101 MHz, Chloroform- $d$ ) $\delta 155.6,155.6,147.1,141.0,134.0,133.4,127.0,126.6,110.2,105.1,74.4,74.2$, 30.9, 27.7, 27.7, 15.4, 12.1. IR $v\left(\mathrm{~cm}^{-1}\right)$ 2919, 2854, 2360, 2341, 1508, 1012, 731. HRMS (ESI-ion trap) calcd for $\mathrm{C}_{11} \mathrm{H}_{16} \mathrm{NO}_{2}{ }^{+}\left([\mathrm{M}+\mathrm{H}]^{+}\right)$194.1176, found 194.1173

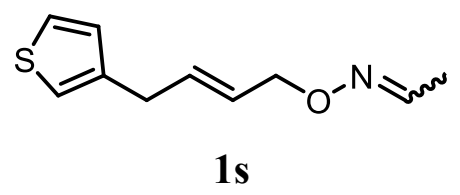

The titled compound was prepared according to General Procedure in $5.0 \mathrm{mmol} \mathrm{scale}$. Yellow oil. $683 \mathrm{mg}, 70 \%$ Yield. Spectroscopic data are given as a mixture of cis and trans isomers (1:1). ${ }^{1} \mathbf{H}$ NMR (400 MHz, Chloroform- $d$ ) $\delta 7.44$ (q, $\left.J=5.9 \mathrm{~Hz}, 0.5 \mathrm{H}\right), 7.14$ (m, 1H), $6.93(\mathrm{~m}, 1 \mathrm{H}), 6.81(\mathrm{~m}, 1 \mathrm{H}), 6.79(\mathrm{~m}, 0.5 \mathrm{H}), 5.92(\mathrm{~m}, 1 \mathrm{H}), 5.78(\mathrm{~m}, 1 \mathrm{H}), 4.59(\mathrm{~m}, 1 \mathrm{H})$, $4.50(\mathrm{~m}, 1 \mathrm{H}), 3.59(\mathrm{~m}, 2 \mathrm{H}), 1.85$ (m, 3H). ${ }^{13} \mathbf{C}$ NMR (101 MHz, Chloroform- $d$ ) $\delta$ 147.1, 143.0, 142.9, 132.6, 132.1, 128.0, 127.7, 127.3, 127.0, 126.7, 125.1, 124.8, 123.8, 123.5, 74.0, 73.7, 32.9, 15.3, 12.1. IR $v\left(\mathrm{~cm}^{-1}\right) 2919,2865,2360,2341,1436,1368,1012,968$, 695. HRMS (ESI-ion trap) calcd for $\mathrm{C}_{10} \mathrm{H}_{14} \mathrm{NOS}^{+}\left([\mathrm{M}+\mathrm{H}]^{+}\right)$196.0791, found 196.0790

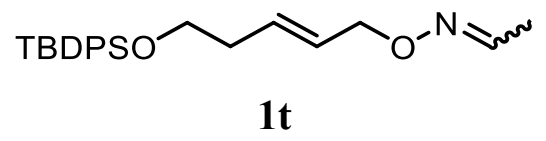

The titled compound was prepared according to General Procedure in $1.9 \mathrm{mmol}$ scale. Colorless oil. $572 \mathrm{mg}, 77 \%$ Yield. Spectroscopic data are given as a mixture of cis and trans isomers (1:1). ${ }^{1} \mathbf{H}$ NMR (400 MHz, Chloroform- $d$ ) $\delta 7.66(\mathrm{~m}, 4 \mathrm{H}), 7.38(\mathrm{~m}, 6 \mathrm{H}), 6.76$ 
$(\mathrm{q}, J=5.5 \mathrm{~Hz}, 0.5 \mathrm{H}), 5.71(\mathrm{~m}, 2 \mathrm{H}), 4.49(\mathrm{~m}, 2 \mathrm{H}), 3.71(\mathrm{~m}, 2 \mathrm{H}), 2.33(\mathrm{~m}, 2 \mathrm{H}), 1.84(\mathrm{~m}$, 3H), 1.05 (s, 9H). ${ }^{13}$ C NMR (101 MHz, Chloroform- $d$ ) $\delta$ 147.0, 135.7, 134.1, 131.8, 131.3, 129.7, 128.1, 127.7, 74.6, 74.3, 63.5, 35.9, 27.0, 19.4, 15.4, 12.1. IR $v\left(\mathrm{~cm}^{-1}\right) 2930,2858$, 2360, 2341, 1428, 1109, 688. HRMS (ESI-ion trap) calcd for $\mathrm{C}_{23} \mathrm{H}_{32} \mathrm{NO}_{2} \mathrm{Si}^{+}\left([\mathrm{M}+\mathrm{H}]^{+}\right)$ 382.2197 , found 382.2184

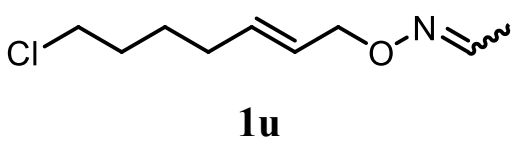

The titled compound was prepared according to General Procedure in $2.0 \mathrm{mmol}$ scale. Colorless oil. $250 \mathrm{mg}, 60 \%$ Yield. Spectroscopic data are given as a mixture of cis and trans isomers (1:1). ${ }^{1} \mathbf{H}$ NMR (400 MHz, Chloroform- $d$ ) $\delta 7.43(\mathrm{q}, J=5.8 \mathrm{~Hz}, 0.5 \mathrm{H}) 6.77$ (q, $J=5.5 \mathrm{~Hz}, 0.5 \mathrm{H}), 5.68(\mathrm{~m}, 2 \mathrm{H}), 4.52(\mathrm{~d}, J=5.7 \mathrm{~Hz}, 1 \mathrm{H}), 4.46(\mathrm{~d}, J=6.0 \mathrm{~Hz}, 1 \mathrm{H}), 3.53$ (m, 2H), $2.10(\mathrm{~m}, 2 \mathrm{H}), 1.85(\mathrm{~m}, 3 \mathrm{H}), 1.80(\mathrm{~m}, 2 \mathrm{H}), 1.55(\mathrm{~m}, 2 \mathrm{H}) .{ }^{13} \mathbf{C}$ NMR (101 MHz, Chloroform- $d$ ) $\delta$ 147.0, 134.7, 134.2, 126.7, 126.4, 74.5, 74.2, 45.0, 32.2, 31.7, 26.3, 26.3, 15.4, 12.1. IR $v\left(\mathrm{~cm}^{-1}\right)$ 2923, 2861, 2360, 2341, 1672, 1637, 1021, 971. HRMS (ESI-ion trap) calcd for $\mathrm{C}_{9} \mathrm{H}_{17} \mathrm{ClNO}^{+}\left([\mathrm{M}+\mathrm{H}]^{+}\right)$190.0993, found 190.0986

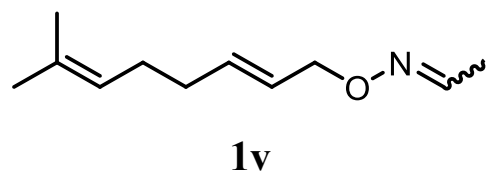

The titled compound was prepared according to General Procedure in $2.1 \mathrm{mmol}$ scale. Colorless oil. $276 \mathrm{mg}, 65 \%$ Yield. Spectroscopic data are given as a mixture of cis and trans isomers (1:1). ${ }^{1} \mathbf{H}$ NMR (400 MHz, Chloroform- $d$ ) $\delta 7.43$ (q, $\left.J=5.9 \mathrm{~Hz}, 0.5 \mathrm{H}\right), 6.76$ (q, $J=5.5 \mathrm{~Hz}, 0.5 \mathrm{H}), 5.74(\mathrm{~m}, 1 \mathrm{H}), 5.65(\mathrm{~m}, 1 \mathrm{H}), 5.11(\mathrm{~m}, 1 \mathrm{H}), 4.49(\mathrm{~m}, 2 \mathrm{H}), 2.08(\mathrm{~m}$, 4H), 1.84 (m, 3H), 1.68 (s, 3H), 1.60 (s, 3H). ${ }^{13}$ C NMR (101 MHz, Chloroform-d) $\delta 146.9$, 135.4, 134.9, 132.0, 132.0, 126.1, 125.7, 123.9, 123.9, 74.7, 74.4, 32.7, 32.7, 27.7, 27.7, 25.8, 17.9, 15.4, 12.1. IR $v\left(\mathrm{~cm}^{-1}\right)$ 2918, 2360, 2341, 1438, 1376, 1019, 969. HRMS (ESIion trap) calcd for $\mathrm{C}_{11} \mathrm{H}_{20} \mathrm{NO}^{+}\left([\mathrm{M}+\mathrm{H}]^{+}\right) 182.1539$, found 182.1534

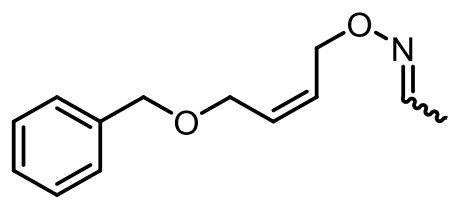




\section{$(Z)-1 w$}

The titled compound was prepared according to General Procedure in $5.0 \mathrm{mmol}$ scale. Colorless oil. $646 \mathrm{mg}, 59 \%$ Yield. Spectroscopic data are given as a mixture of cis and trans isomers (1.2:1). ${ }^{1} \mathbf{H}$ NMR (400 MHz, Chloroform- $d$ ) $\delta 7.42(\mathrm{q}, J=5.9 \mathrm{~Hz}, 0.6 \mathrm{H})$, $7.34(\mathrm{~m}, 5 \mathrm{H}), 6.77$ (q, $J=5.5 \mathrm{~Hz}, 0.4 \mathrm{H}), 5.81(\mathrm{~m}, 2 \mathrm{H}), 4.61(\mathrm{~m}, 2 \mathrm{H}), 4.52(\mathrm{~m}, 2 \mathrm{H}), 4.14$ (m, 2H), 1.84 (t, $J=5.4 \mathrm{~Hz}, 3 \mathrm{H}) .{ }^{13} \mathbf{C}$ NMR (101 MHz, Chloroform- $d$ ) $\delta 147.3,138.3,130.1$, $129.8,129.0,128.7,128.5,127.9,127.8,72.4,72.4,69.5,69.1,66.0,66.0,15.4,12.0$. IR $v\left(\mathrm{~cm}^{-1}\right)$ 2921, 2856, 2360, 2341, 1739, 1454, 1027. HRMS (ESI-ion trap) calcd for $\mathrm{C}_{13} \mathrm{H}_{18} \mathrm{NO}_{2}{ }^{+}\left([\mathrm{M}+\mathrm{H}]^{+}\right)$220.1332, found 220.1326

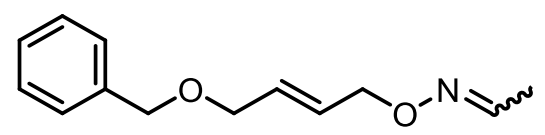

\section{$(E)-1 \mathbf{w}$}

The titled compound was prepared according to General Procedure in $5.0 \mathrm{mmol}$ scale. Colorless oil. $762 \mathrm{mg}, 70 \%$ Yield. Spectroscopic data are given as a mixture of cis and trans isomers (1.2:1) ${ }^{\mathbf{1}} \mathbf{H}$ NMR (400 MHz, Chloroform- $d$ ) $\delta 7.45(\mathrm{~m}, 0.4 \mathrm{H}), 7.32(\mathrm{~m}, 5 \mathrm{H})$, $6.78(\mathrm{q}, J=5.5 \mathrm{~Hz}, 0.6 \mathrm{H}), 5.90(\mathrm{~m}, 2 \mathrm{H}), 4.61(\mathrm{~d}, J=5.3 \mathrm{~Hz}, 1 \mathrm{H}), 4.54(\mathrm{~m}, 3 \mathrm{H}), 4.06(\mathrm{~m}$, 2H), 1.85 (m, 3H). ${ }^{13}$ C NMR (101 MHz, Chloroform- $d$ ) $\delta$ 147.2, 147.2, 138.4, 130.2, 129.7, 129.3, 129.0, 128.5, 127.9, 127.9, 127.7, 73.7, 73.4, 72.3, 72.3, 70.2, 70.2, 15.3, 12.0. IR $v\left(\mathrm{~cm}^{-1}\right) .2852,1737,1364,736,679$. HRMS (ESI-ion trap) calcd for $\mathrm{C}_{13} \mathrm{H}_{18} \mathrm{NO}_{2}{ }^{+}\left([\mathrm{M}+\mathrm{H}]^{+}\right)$ 220.1332 , found 220.1326

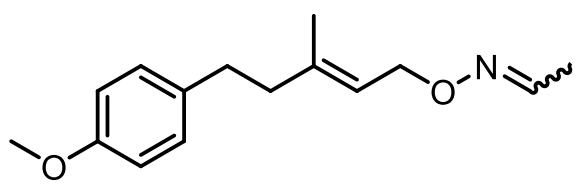

$1 \mathbf{x}$

The titled compound was prepared according to General Procedure in $10 \mathrm{mmol}$ scale. Colorless oil. $1.6 \mathrm{~g}, 66 \%$ Yield. Spectroscopic data are given as a mixture of cis and trans isomers (1.2:1). ${ }^{1} \mathbf{H}$ NMR (400 MHz, Chloroform- $d$ ) $\delta 7.43$ (q, $\left.J=5.9 \mathrm{~Hz}, 0.4 \mathrm{H}\right), 7.10(\mathrm{~m}$, 2H), $6.82(\mathrm{~m}, 2 \mathrm{H}), 6.77$ (q, $J=5.6 \mathrm{~Hz}, 0.6 \mathrm{H}), 5.44(\mathrm{~m}, 1 \mathrm{H}), 4.56(\mathrm{~m}, 2 \mathrm{H}), 3.79$ (s, 3H), 2.69 (m, 2H), 2.32 (m, 2H), 1.85 (m, 3H), 1.75 (s, 3H). ${ }^{13} \mathbf{C}$ NMR (101 MHz, Chloroform- 
d) $\delta 157.9,146.9,146.9,146.8,141.0,140.2,134.3,134.1,129.4,129.4,129.3,121.7$, $121.2,120.7,120.1,113.9,113.9,70.5,70.1,70.1,69.7,55.4,41.9,41.9,34.8,34.7,33.8$, $33.8,33.6,23.8,16.9,16.9,15.4,12.1$. IR $v\left(\mathrm{~cm}^{-1}\right) 2921,2361,2341,1511,1244,1035$, 823. HRMS (ESI-ion trap) calcd for $\mathrm{C}_{15} \mathrm{H}_{22} \mathrm{NO}_{2}{ }^{+}\left([\mathrm{M}+\mathrm{H}]^{+}\right)$248.1645, found 248.1637

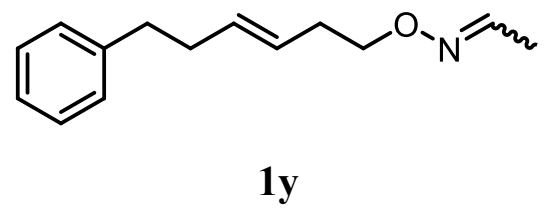

The titled compound was prepared according to General Procedure in $2.8 \mathrm{mmol}$ scale. Colorless oil. $456 \mathrm{mg}, 74 \%$ Yield. Spectroscopic data are given as a mixture of cis and trans isomers (1:1). ${ }^{1} \mathbf{H}$ NMR (400 MHz, Chloroform- $d$ ) $\delta 7.41$ (q, $\left.J=5.8 \mathrm{~Hz}, 0.5 \mathrm{H}\right), 7.29$ (m, 2H), $7.17(\mathrm{~m}, 3 \mathrm{H}), 6.75(\mathrm{q}, J=5.5 \mathrm{~Hz}, 1 \mathrm{H}), 5.55(\mathrm{~m}, 1 \mathrm{H}), 5.46(\mathrm{~m}, 1 \mathrm{H}), 4.03(\mathrm{~m}, 2 \mathrm{H})$, $2.68(\mathrm{~m}, 2 \mathrm{H}), 2.33(\mathrm{~m}, 4 \mathrm{H}), 1.84(\mathrm{~m}, 3 \mathrm{H}) .{ }^{13} \mathbf{C}$ NMR (101 MHz, Chloroform- $d$ ) $\delta 147.0$, 147.0, 142.5, 135.2, 134.7, 128.6, 128.5, 128.4, 126.5, 126.1, 125.8, 74.6, 74.3, 35.5, 32.0, 30.8, 30.8, 15.4, 12.1. IR $v\left(\mathrm{~cm}^{-1}\right)$ 2923, 2361, 2341, 1474, 1044, 669. HRMS (ESI-ion trap) calcd for $\mathrm{C}_{14} \mathrm{H}_{20} \mathrm{NO}^{+}\left([\mathrm{M}+\mathrm{H}]^{+}\right) 218.1539$, found 218.1534

\section{General procedure for Ir-catalyzed hydroalkynylation}

In an Ar-filled glovebox, $\operatorname{Ir}(\mathrm{COD})_{2} \mathrm{OTf}(5.6 \mathrm{mg}, 10 \mathrm{~mol} \%)$, Ph-BPE (6.1 mg, $12 \mathrm{~mol} \%$ ) and unsaturated oxime $(0.10 \mathrm{mmol})$ were weighed into a Schlenk tube. Then $0.40 \mathrm{~mL}$ DCE and triisopropylsilylacetylene $(55 \mathrm{mg}, 0.30 \mathrm{mmol}$ ) were added via syringes. The vial was capped with a Teflon-lined screw cap and removed from the glovebox. Subsequently the vial was put in a preheated oil bath $\left(60^{\circ} \mathrm{C}\right)$. After $24 \mathrm{~h}$, the vial was cool to room temperature, and the reaction mixture was purified by column chromatography with ethyl acetate/hexanes as eluent.

\section{Characterization of products}




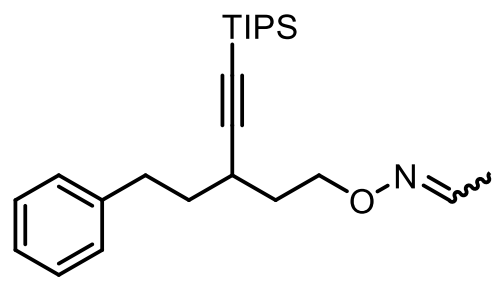

3a

Following the general procedure, oxime 1a $(20.3 \mathrm{mg}, 0.100 \mathrm{mmol})$ was converted to the alkynylation product. Purification by column chromatography with EtOAc/Hexane as eluent gave the product $3 \mathbf{a}$ (31.6 $\mathrm{mg}$, 82\% yield) as a light-yellow oil.

Following the general procedure, oxime 1a $(203 \mathrm{mg}, 1.00 \mathrm{mmol})$ was converted to the alkynylation product. Purification by column chromatography with EtOAc/Hexane as eluent gave the product 3a (346 mg, 90\% yield) as a light-yellow oil.

Spectroscopic data are given as a mixture of cis and trans isomers (1:1). ${ }^{1} \mathbf{H}$ NMR (400 MHz, Chloroform- $d$ ) $\delta 7.39$ (q, $J=5.8 \mathrm{~Hz}, 0.5 \mathrm{H}$ ), 7.29 (m, 2H), 7.21 (m, 3H), 6.74 (q, $J=$ $5.5 \mathrm{~Hz}, 0.5 \mathrm{H}), 4.22(\mathrm{~m}, 2 \mathrm{H}), 2.90(\mathrm{~m}, 1 \mathrm{H}), 2.78(\mathrm{~m}, 1 \mathrm{H}), 2.56(\mathrm{~m}, 1 \mathrm{H}), 1.82(\mathrm{~m}, 7 \mathrm{H}), 1.11$ (m, 21H). ${ }^{13}$ C NMR (101 MHz, Chloroform- $d$ ) $\delta$ 146.8, 146.7, 142.3, 142.2, 128.7, 128.5, $128.5,125.9,111.3,111.3,82.2,82.2,71.7,71.5,37.5,37.4,34.7,33.8,33.7,29.3,29.2$, 18.8, 15.3, 11.9, 11.4. IR $v\left(\mathrm{~cm}^{-1}\right) 2941,2864,2360,2341,2164,1461,862,675$. HRMS (ESI-ion trap) calcd for $\mathrm{C}_{24} \mathrm{H}_{40} \mathrm{NOSi}^{+}\left([\mathrm{M}+\mathrm{H}]^{+}\right) 386.2874$, found 386.2861

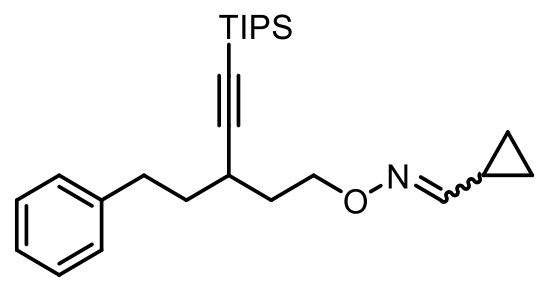

3b

Following the general procedure, oxime with $\mathbf{1 b}(22.9 \mathrm{mg}, 0.100 \mathrm{mmol})$ was converted to the alkynylation product. Purification by column chromatography with EtOAc/Hexane as eluent gave the product $\mathbf{3 b}$ (15.6 mg, 38\% yield) as a colorless oil. Spectroscopic data are given as a mixture of cis and trans isomers (1:1). ${ }^{1} \mathbf{H}$ NMR (400 MHz, Chloroform- $d$ ) $\delta 7.28(\mathrm{~m}, 2 \mathrm{H}), 7.20(\mathrm{~m}, 3 \mathrm{H}), 6.85(\mathrm{~d}, J=8.4 \mathrm{~Hz}, 1 \mathrm{H}), 4.17(\mathrm{~m}, 2 \mathrm{H}), 2.88(\mathrm{~m}, 1 \mathrm{H}), 2.76$ 
$(\mathrm{m}, 1 \mathrm{H}), 2.54(\mathrm{~m}, 1 \mathrm{H}), 1.86(\mathrm{~m}, 1 \mathrm{H}), 1.76(\mathrm{~m}, 3 \mathrm{H}), 1.59(\mathrm{~m}, 1 \mathrm{H}), 1.07(\mathrm{~m}, 21 \mathrm{H}), 0.85(\mathrm{~m}$, 2H), $0.59(\mathrm{~m}, 2 \mathrm{H}) .{ }^{13} \mathbf{C}$ NMR (101 MHz, Chloroform- $d$ ) $\delta 154.1,142.3,128.7,128.5,125.9$, 111.3, 82.2, 71.5, 37.5, 34.7, 33.8, 29.3, 18.9, 11.4, 11.1, 5.7, 5.7. IR $v\left(\mathrm{~cm}^{-1}\right) 2941,2864$, 2360, 2341, 1382, 1058, 883. HRMS (ESI-ion trap) calcd for $\mathrm{C}_{26} \mathrm{H}_{42} \mathrm{NOSi}^{+}\left([\mathrm{M}+\mathrm{H}]^{+}\right)$ 412.3030 , found 412.3013

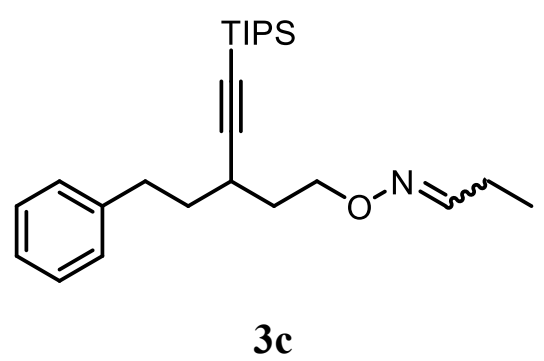

Following the general procedure, oxime $1 \mathrm{c}(21.7 \mathrm{mg}, 0.100 \mathrm{mmol})$ was converted to the alkynylation product. Purification by column chromatography with EtOAc/Hexane as eluent gave the product $3 \mathbf{c}(23.5 \mathrm{mg}, 59 \%$ yield) as a colorless oil. Spectroscopic data are given as a mixture of cis and trans isomers (1.2:1). ${ }^{1} \mathbf{H}$ NMR (400 MHz, Chloroform- $d$ ) $\delta$ $7.36(\mathrm{t}, J=6.0 \mathrm{~Hz}, 0.6 \mathrm{H}), 7.28(\mathrm{~m}, 2 \mathrm{H}), 7.20(\mathrm{~m}, 3 \mathrm{H}), 6.60(\mathrm{t}, J=5.3 \mathrm{~Hz}, 0.4 \mathrm{H}), 4.20(\mathrm{~m}$, $2 \mathrm{H}), 2.89(\mathrm{~m}, 1 \mathrm{H}), 2.77(\mathrm{~m}, 1 \mathrm{H}), 2.55(\mathrm{~m}, 1 \mathrm{H}), 2.29(\mathrm{~m}, 2 \mathrm{H}), 1.87(\mathrm{~m}, 1 \mathrm{H}), 1.78(\mathrm{~m}, 2 \mathrm{H})$, 1.08 (m, 24H). ${ }^{13} \mathbf{C}$ NMR (101 MHz, Chloroform- $d$ ) $\delta$ 153.2, 151.9, 142.3, 142.3, 128.7, 128.6, 128.5, 128.5, 125.9, 111.3, 111.3, 82.2, 71.8, 71.5, 37.5, 37.4, 34.7, 33.8, 33.8, 29.3, 29.2, 23.2, 19.3, 18.8, 11.4, 11.3, 10.8. IR $v\left(\mathrm{~cm}^{-1}\right) 2941,2865,2361,2341,2164,1459$, 1059, 883. HRMS (ESI-ion trap) calcd for $\mathrm{C}_{25} \mathrm{H}_{42} \mathrm{NOSi}^{+}\left([\mathrm{M}+\mathrm{H}]^{+}\right) 400.3030$, found 400.3017

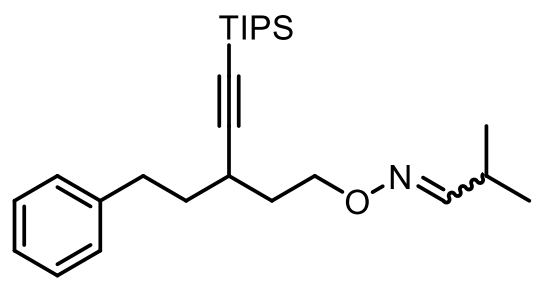

3d

Following the general procedure, oxime $1 \mathbf{d}(23.1 \mathrm{mg}, 0.100 \mathrm{mmol})$ was converted to the alkynylation product. Purification by column chromatography with EtOAc/Hexane as eluent gave the product 3d (19.8 mg, 48\% yield) as a colorless oil. Spectroscopic data are 
given as a mixture of cis and trans isomers (4:1). ${ }^{1} \mathbf{H}$ NMR (400 MHz, Chloroform- $d$ ) $\delta$ $7.26(\mathrm{~m}, 2 \mathrm{H}), 7.20$ (m, 3H), 6.43 (d, $J=7.2 \mathrm{~Hz}, 0.2 \mathrm{H}), 4.18(\mathrm{~m}, 2 \mathrm{H}), 2.91(\mathrm{~m}, 0.2 \mathrm{H}), 2.89$ (m, 1H), $2.76(\mathrm{~m}, 1 \mathrm{H}), 2.49(\mathrm{~m}, 2 \mathrm{H}), 1.80(\mathrm{~m}, 4 \mathrm{H}), 1.08(\mathrm{~m}, 28 \mathrm{H}) .{ }^{13} \mathbf{C}$ NMR $(101 \mathrm{MHz}$, Chloroform- $d$ ) $\delta 157.4,155.8,142.3,128.7,128.5,125.9,111.3,82.2,71.7,71.4,37.5$, $37.4,34.7,33.8,33.8,29.5,29.3,29.2,25.2,20.3,20.2,19.9,18.8,11.5 . \mathbf{I R} v\left(\mathrm{~cm}^{-1}\right) 2959$, 2865, 2361, 2341, 2164, 1496, 1060, 883, 676. HRMS (ESI-ion trap) calcd for $\mathrm{C}_{26} \mathrm{H}_{44} \mathrm{NOSi}^{+}\left([\mathrm{M}+\mathrm{H}]^{+}\right)$414.3187, found 414.3176

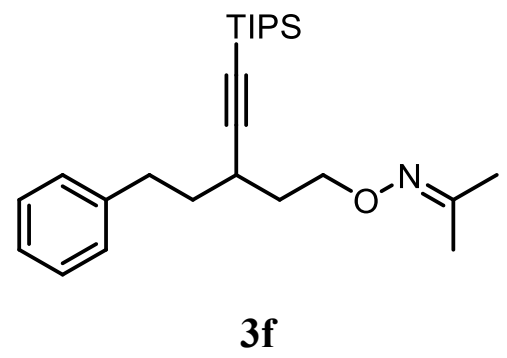

Following the general procedure, oxime 1f $(43.4 \mathrm{mg}, 0.200 \mathrm{mmol})$ was converted to the alkynylation product. Purification by column chromatography with EtOAc/Hexane as eluent gave the product $\mathbf{3 f}$ ( $8.40 \mathrm{mg}, 10 \%$ yield) as a colorless oil. ${ }^{1} \mathbf{H}$ NMR (400 MHz, Chloroform- $d$ ) $\delta 7.28(\mathrm{~d}, J=7.5 \mathrm{~Hz}, 2 \mathrm{H}), 7.25(\mathrm{~m}, 3 \mathrm{H}), 4.17(\mathrm{~m}, 2 \mathrm{H}), 2.88(\mathrm{~m}, 1 \mathrm{H}), 2.76(\mathrm{~m}$, 1H), $2.54(\mathrm{~m}, 1 \mathrm{H}), 1.82(\mathrm{~m}, 10 \mathrm{H}), 1.09(\mathrm{~m}, 21 \mathrm{H}) .{ }^{13} \mathbf{C}$ NMR (101 MHz, Chloroform- $\left.d\right) \delta$ 154.7, 142.3, 128.7, 128.5, 125.9, 111.5, 82.1, 71.2, 37.4, 34.8, 33.8, 29.3, 22.0, 18.8, 15.7, 11.5. IR $v\left(\mathrm{~cm}^{-1}\right)$ 2942, 2864, 2360, 2341, 1558, 1070, 919. HRMS (ESI-ion trap) calcd for $\mathrm{C}_{25} \mathrm{H}_{42} \mathrm{NOSi}^{+}\left([\mathrm{M}+\mathrm{H}]^{+}\right) 400.3030$, found 400.3014

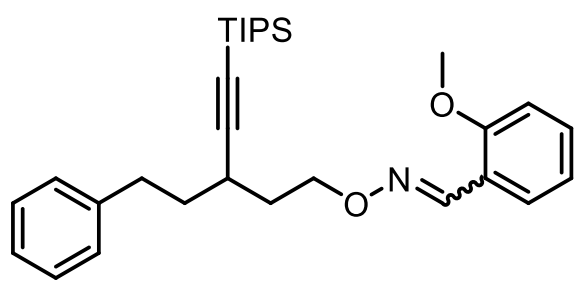

$3 \mathbf{h}$

Following the general procedure, oxime $1 \mathrm{~h}(29.5 \mathrm{mg}, 0.100 \mathrm{mmol})$ was converted to the alkynylation product. Purification by column chromatography with EtOAc/Hexane as eluent gave the product $\mathbf{3 h}$ (14.8 $\mathrm{mg}, 31 \%$ yield) as a colorless oil. Spectroscopic data are given as a mixture of $c i s$ and trans isomers (4:1). ${ }^{1} \mathbf{H}$ NMR (400 MHz, Chloroform- $d$ ) $\delta$ 
$7.28(\mathrm{~m}, 2 \mathrm{H}), 7.20(\mathrm{~m}, 3 \mathrm{H}), 6.85(\mathrm{~m}, 2 \mathrm{H}), 4.17(\mathrm{~m}, 2 \mathrm{H}), 3.84(\mathrm{~m}, 3 \mathrm{H}), 2.91(\mathrm{~m}, 1 \mathrm{H}), 2.75$ $(\mathrm{m}, 1 \mathrm{H}), 2.54(\mathrm{~m}, 1 \mathrm{H}), 1.85(\mathrm{~m}, 4 \mathrm{H}), 1.76(\mathrm{~m}, 1 \mathrm{H}), 1.11(\mathrm{~m}, 21 \mathrm{H}), 0.84(\mathrm{~m}, 2 \mathrm{H}), 0.59(\mathrm{~m}$, 2H). ${ }^{13}$ C NMR (101 MHz, Chloroform- $d$ ) $\delta 157.6,144.7,142.3,131.1,128.7,128.5,126.5$, $125.9,121.2,120.9,111.4,111.2,82.3,72.2,55.7,37.5,34.9,33.8,29.4,19.0,18.9,11.5$. IR $v\left(\mathrm{~cm}^{-1}\right)$ 2940, 2863, 2361, 2341, 2164, 1464, 1251, 752. HRMS (ESI-ion trap) calcd for $\mathrm{C}_{30} \mathrm{H}_{44} \mathrm{NO}_{2} \mathrm{Si}^{+}\left([\mathrm{M}+\mathrm{H}]^{+}\right)$478.3136, found 478.3120

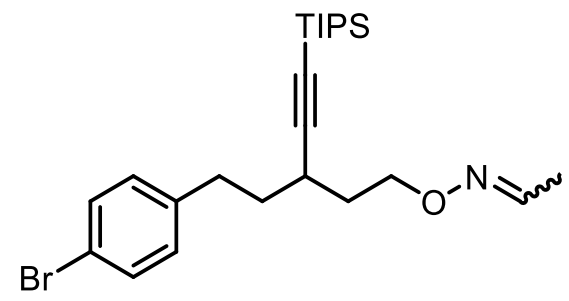

$3 \mathbf{i}$

Following the general procedure, oxime $1 \mathbf{i}(28.1 \mathrm{mg}, 0.100 \mathrm{mmol})$ was converted to the alkynylation product. Purification by column chromatography with EtOAc/Hexane as eluent gave the product $3 \mathbf{i}$ (39.4 $\mathrm{mg}, 85 \%$ yield) as a colorless oil. Spectroscopic data are given as a mixture of cis and trans isomers (1:1). ${ }^{1} \mathbf{H}$ NMR (400 MHz, Chloroform- $\left.d\right) \delta 7.39(\mathrm{~m}$, 2.5H), $7.07(\mathrm{~m}, 2 \mathrm{H}), 6.73$ (q, J=5.5 Hz, 0.5H), $4.17(\mathrm{~m}, 2 \mathrm{H}), 2.85(\mathrm{~m}, 1 \mathrm{H}), 2.73(\mathrm{~m}, 1 \mathrm{H})$, $2.51(\mathrm{~m}, 1 \mathrm{H}), 1.81(\mathrm{~m}, 6 \mathrm{H}), 1.09(\mathrm{~s}, 21 \mathrm{H}) .{ }^{13} \mathbf{C}$ NMR $(101 \mathrm{MHz}$, Chloroform- $d$ ) $\delta 146.9$, 146.7, 141.2, 141.1, 131.5, 130.4, 119.7, 111.1, 111.0, 82.5, 82.5, 71.6, 71.4, 37.2, 37.1, 34.7, 33.1, 33.1, 29.2, 29.1, 18.8, 15.4, 11.9, 11.4. IR $v\left(\mathrm{~cm}^{-1}\right)$ 2942, 2864, 2360, 2341, 2164, 1489, 1462, 996, 863. HRMS (ESI-ion trap) calcd for $\mathrm{C}_{24} \mathrm{H}_{39} \mathrm{BrNOSi}^{+}\left([\mathrm{M}+\mathrm{H}]^{+}\right)$ 464.1979 , found 464.1962

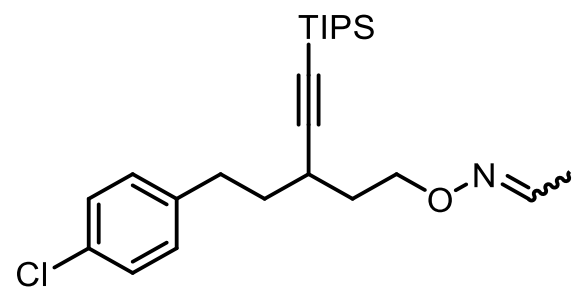

$3 \mathbf{j}$

Following the general procedure, oxime $\mathbf{1 j}$ ( $23.7 \mathrm{mg}, 0.100 \mathrm{mmol}$ ) was converted to the alkynylation product. Purification by column chromatography with EtOAc/Hexane as eluent gave the product $3 \mathbf{j}$ ( $28.9 \mathrm{mg}, 69 \%$ yield) as a colorless oil. Spectroscopic data are given 
as a mixture of cis and trans isomers (1:1). ${ }^{1} \mathbf{H}$ NMR $(400 \mathrm{MHz}$, Chloroform- $d$ ) $\delta 7.40$ (q, $J=5.8 \mathrm{~Hz}, 0.5 \mathrm{H}), 7.26(\mathrm{~m}, 2 \mathrm{H}), 7.15(\mathrm{~m}, 2 \mathrm{H}), 6.75(\mathrm{q}, J=5.5 \mathrm{~Hz}, 1 \mathrm{H}), 4.24(\mathrm{~m}, 2 \mathrm{H}), 2.87$ $(\mathrm{m}, 1 \mathrm{H}), 2.76(\mathrm{~m}, 1 \mathrm{H}), 2.54(\mathrm{~m}, 1 \mathrm{H}), 1.83(\mathrm{~m}, 7 \mathrm{H}), 1.11(\mathrm{~s}, 21 \mathrm{H}) .{ }^{13} \mathbf{C}$ NMR $(101 \mathrm{MHz}$, Chloroform- $d$ ) $\delta 146.9,146.7,140.6,140.6,131.7,130.0,128.6,111.1,111.0,82.5,82.4$, 71.6, 71.4, 37.2, 37.1, 34.7, 33.1, 33.1, 29.2, 29.1, 18.8, 15.4, 11.9, 11.4. IR $v\left(\mathrm{~cm}^{-1}\right) 2941$, 2890, 2864, 2361, 2341, 2164, 1492, 863. HRMS (ESI-ion trap) calcd for $\mathrm{C}_{24} \mathrm{H}_{39} \mathrm{CINOSi}{ }^{+}$ $\left([\mathrm{M}+\mathrm{H}]^{+}\right) 420.2484$, found 420.2472

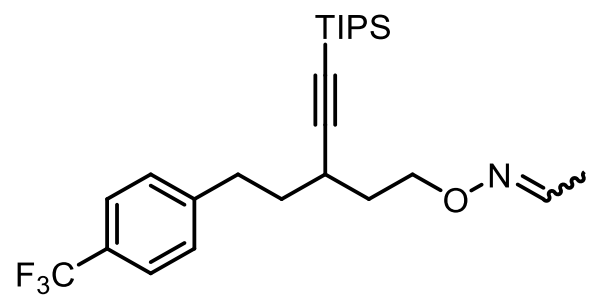

$3 \mathbf{k}$

Following the general procedure, oxime $1 \mathrm{k}(27.1 \mathrm{mg}, 0.100 \mathrm{mmol})$ was converted to the alkynylation product. Purification by column chromatography with EtOAc/Hexane as eluent gave the product $3 \mathbf{k}(38.1 \mathrm{mg}, 84 \%$ yield) as a colorless oil. Spectroscopic data are given as a mixture of cis and trans isomers (1:1). ${ }^{1} \mathbf{H}$ NMR $(400 \mathrm{MHz}$, Chloroform- $d$ ) $\delta$ $7.53(\mathrm{~m}, 2 \mathrm{H}), 7.37$ (q, $J=5.9 \mathrm{~Hz}, 0.5 \mathrm{H}), 7.31(\mathrm{~m}, 2 \mathrm{H}), 6.73(\mathrm{~m}, 0.5 \mathrm{H}), 4.20(\mathrm{~m}, 2 \mathrm{H}), 2.94$ $(\mathrm{m}, 1 \mathrm{H}), 2.83(\mathrm{~m}, 1 \mathrm{H}), 2.52(\mathrm{~m}, 1 \mathrm{H}), 1.80(\mathrm{~m}, 7 \mathrm{H}), 1.09(\mathrm{~s}, 21 \mathrm{H}) .{ }^{13} \mathbf{C}$ NMR $(101 \mathrm{MHz}$, Chloroform- $d$ ) $\delta 147.0,146.8,146.3,129.0,128.5,128.2,125.9,125.5,125.4,125.4,123.2$, $110.9,110.9,82.6,71.6,71.3,37.0,36.9,34.7,33.6,33.6,29.2,29.1,18.8,15.3,11.9,11.4$. IR $v\left(\mathrm{~cm}^{-1}\right)$ 2943, 2866, 2361, 2341, 1772, 1733, 883, 750. HRMS (ESI-ion trap) calcd for $\mathrm{C}_{25} \mathrm{H}_{39} \mathrm{~F}_{3} \mathrm{NOSi}^{+}\left([\mathrm{M}+\mathrm{H}]^{+}\right) 454.2748$, found 454.2728

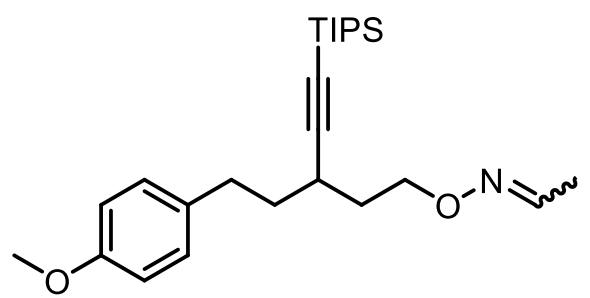

31 
Following the general procedure, oxime $11(23.3 \mathrm{mg}, 0.100 \mathrm{mmol})$ was converted to the alkynylation product. Purification by column chromatography with EtOAc/Hexane as eluent gave the product 31 (36.5 mg, 88\% yield) as a colorless oil. Spectroscopic data are given as a mixture of cis and trans isomers (1:1). ${ }^{1} \mathbf{H}$ NMR $(400 \mathrm{MHz}$, Chloroform- $d$ ) $\delta 7.40$ (q, $J=5.8 \mathrm{~Hz}, 0.5 \mathrm{H}), 7.14(\mathrm{~m}, 2 \mathrm{H}), 6.85(\mathrm{~m}, 2 \mathrm{H}), 6.75(\mathrm{q}, J=5.5 \mathrm{~Hz}, 0.5 \mathrm{H}), 4.23(\mathrm{~m}, 2 \mathrm{H})$, $3.81(\mathrm{~s}, 3 \mathrm{H}), 2.84(\mathrm{~m}, 1 \mathrm{H}), 2.74(\mathrm{~m}, 1 \mathrm{H}), 2.55(\mathrm{~m}, 1 \mathrm{H}), 1.81(\mathrm{~m}, 7 \mathrm{H}), 1.12(\mathrm{~s}, 21 \mathrm{H}) .{ }^{13} \mathrm{C}$ NMR (101 MHz, Chloroform- $d$ ) $\delta$ 157.9, 146.9, 146.7, 134.4, 134.3, 129.5, 113.9, 111.4, $111.4,82.2,82.1,71.8,71.5,55.4,37.7,37.6,34.7,32.8,32.8,29.2,29.2,18.8,15.4,12.0$, 11.4, 11.4. IR $v\left(\mathrm{~cm}^{-1}\right)$ 2941, 2864, 2361, 2341, 1513, 1246, 750. HRMS (ESI-ion trap) calcd for $\mathrm{C}_{25} \mathrm{H}_{42} \mathrm{NO}_{2} \mathrm{Si}^{+}\left([\mathrm{M}+\mathrm{H}]^{+}\right) 416.2979$, found 416.2965

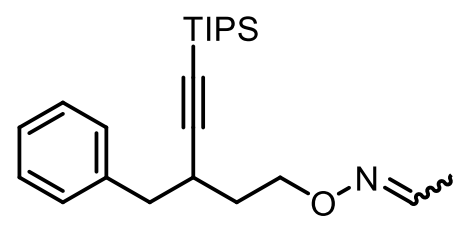

$3 \mathbf{m}$

Following the general procedure, oxime $1 \mathbf{m}(18.9 \mathrm{mg}, 0.100 \mathrm{mmol})$ was converted to the alkynylation product. Purification by column chromatography with EtOAc/Hexane as eluent gave the product $3 \mathrm{~m}$ (33.4 mg, 90\% yield) as a colorless oil. Spectroscopic data are given as a mixture of cis and trans isomers (1:1). ${ }^{1} \mathbf{H}$ NMR (400 MHz, Chloroform- $d$ ) $\delta$ $7.40(\mathrm{q}, J=5.9 \mathrm{~Hz}, 0.5 \mathrm{H}), 7.27(\mathrm{~m}, 4 \mathrm{H}), 7.20(\mathrm{~m}, 1 \mathrm{H}), 6.75(\mathrm{~m}, 0.5 \mathrm{H}), 4.22(\mathrm{~m}, 2 \mathrm{H}), 2.81$ $(\mathrm{m}, 3 \mathrm{H}), 1.93(\mathrm{~m}, 1 \mathrm{H}), 1.84(\mathrm{~m}, 3 \mathrm{H}), 1.74(\mathrm{~m}, 1 \mathrm{H}), 1.03(\mathrm{~s}, 21 \mathrm{H}) .{ }^{13} \mathbf{C}$ NMR $(101 \mathrm{MHz}$, Chloroform- $d$ ) $\delta$ 146.9, 146.7, 139.5, 129.5, 128.2, 128.2, 126.3, 110.8, 82.7, 71.8, 71.4, 41.7, 34.3, 34.3, 31.7, 31.7, 18.7, 15.4, 12.0, 11.4. IR $v\left(\mathrm{~cm}^{-1}\right)$ 2941, 2864, 2360, 2342, 2165, 1462, 863, 676. HRMS (ESI-ion trap) calcd for $\mathrm{C}_{23} \mathrm{H}_{38} \mathrm{NOSi}^{+}\left([\mathrm{M}+\mathrm{H}]^{+}\right) 372.2717$, found 372.2705

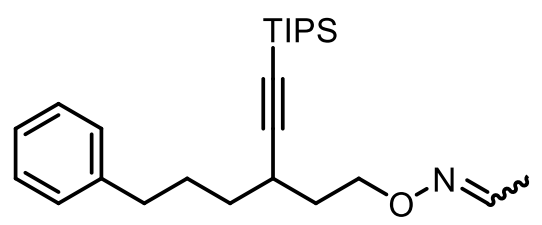

$3 n$ 
Following the general procedure, oxime $1 \mathrm{n}(21.7 \mathrm{mg}, 0.100 \mathrm{mmol})$ was converted to the alkynylation product. Purification by column chromatography with EtOAc/Hexane as eluent gave the product $3 \mathbf{n}$ (30.3 mg, 76\% yield) as a colorless oil. ${ }^{1} \mathbf{H}$ NMR (400 MHz, Chloroform- $d$ ) $\delta 7.26(\mathrm{~m}, 2 \mathrm{H}), 7.17(\mathrm{~m}, 3 \mathrm{H}), 6.74(\mathrm{q}, J=5.5 \mathrm{~Hz}, 1 \mathrm{H}), 4.23(\mathrm{~m}, 2 \mathrm{H}), 2.62(\mathrm{~m}$, $3 \mathrm{H}), 1.81(\mathrm{~m}, 7 \mathrm{H}), 1.51(\mathrm{~m}, 2 \mathrm{H}), 1.06(\mathrm{~s}, 21 \mathrm{H}) .{ }^{13} \mathbf{C}$ NMR (101 MHz, Chloroform- $d$ ) $\delta$ 146.8, 142.6, 128.5, 128.4, 125.8, 111.6, 81.7, 71.8, 35.8, 34.9, 34.8, 29.5, 29.1 18.8, 12.0, 11.4. IR $v\left(\mathrm{~cm}^{-1}\right) 2943,2865,2360,2253,1636,1375,1039,919$. HRMS (ESI-ion trap) calcd for $\mathrm{C}_{25} \mathrm{H}_{42} \mathrm{NOSi}^{+}\left([\mathrm{M}+\mathrm{H}]^{+}\right) 400.3030$, found 400.3016

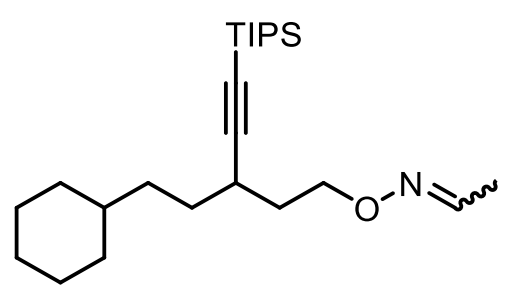

30

Following the general procedure, oxime $10(20.9 \mathrm{mg}, 0.100 \mathrm{mmol})$ was converted to the alkynylation product. Purification by column chromatography with EtOAc/Hexane as eluent gave the product $3 \mathrm{o}(25.4 \mathrm{mg}, 65 \%$ yield) as a colorless oil. Spectroscopic data are given as a mixture of cis and trans isomers (1:1). ${ }^{1} \mathbf{H}$ NMR (400 MHz, Chloroform- $d$ ) $\delta$ $7.40(\mathrm{q}, J=5.9 \mathrm{~Hz}, 0.5 \mathrm{H}), 6.74(\mathrm{q}, J=5.5 \mathrm{~Hz}, 0.5 \mathrm{H}), 4.22(\mathrm{~m}, 2 \mathrm{H}), 2.48(\mathrm{~m}, 1 \mathrm{H}), 1.84(\mathrm{~m}$, 3H), $1.68(\mathrm{~m}, 4 \mathrm{H}), 1.44(\mathrm{~m}, 3 \mathrm{H}), 1.20(\mathrm{~m}, 7 \mathrm{H}), 1.06(\mathrm{~s}, 21 \mathrm{H}), 0.89(\mathrm{~m}, 3 \mathrm{H}) .{ }^{13} \mathbf{C}$ NMR $(101$ MHz, Chloroform- $d$ ) $\delta 146.8,146.6,112.0,112.0,81.4,71.9,71.6,37.6,35.0,34.7,33.8$, $33.3,32.8,32.7,29.8,29.8,26.9,26.5,26.5,18.8,15.4,12.0,11.4$. IR $v\left(\mathrm{~cm}^{-1}\right) 2921,2863$, 2361, 2341, 2165, 2030, 1462, 861, 661. HRMS (ESI-ion trap) calcd for $\mathrm{C}_{24} \mathrm{H}_{46} \mathrm{NOSi}^{+}$ $\left([\mathrm{M}+\mathrm{H}]^{+}\right) 392.3343$, found 392.3326

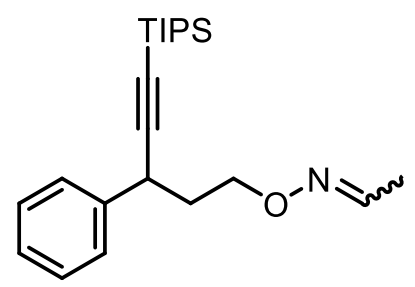

$3 p$ 
Following the general procedure, oxime $1 \mathbf{p}(17.5 \mathrm{mg}, 0.100 \mathrm{mmol})$ was converted to the alkynylation product. Purification by column chromatography with EtOAc/Hexane as eluent gave the product $3 \mathbf{p}$ ( $9.60 \mathrm{mg}, 27 \%$ yield) as a colorless oil. Spectroscopic data are given as a mixture of cis and trans isomers (1:1). ${ }^{1} \mathbf{H}$ NMR (400 MHz, Chloroform- $d$ ) $\delta$ 7.40 (m, 2.5H), 7.32 (m, 2H), $7.22(\mathrm{~m}, 1 \mathrm{H}), 6.76$ (q, $J=5.5 \mathrm{~Hz}, 1 \mathrm{H}), 4.19(\mathrm{~m}, 2 \mathrm{H}), 3.86$ (m, 1H), $2.10(\mathrm{~m}, 2 \mathrm{H}), 1.84(\mathrm{~m}, 3 \mathrm{H}), 1.09(\mathrm{~s}, 21 \mathrm{H}) .{ }^{13} \mathbf{C}$ NMR (101 MHz, Chloroform- $d$ ) $\delta$ 146.9, 146.7, 138.5, 128.5, 127.9, 127.6, 109.0, 82.5, 73.4, 73.3, 73.2, 73.2, 71.5, 71.2, 31.5, 31.5, 30.7, 30.6, 18.8, 15.4, 12.0, 11.4, 11.4. IR $v\left(\mathrm{~cm}^{-1}\right)$ 2941, 2864, 2360, 2341, 1541, 750, 669. HRMS (ESI-ion trap) calcd for $\mathrm{C}_{22} \mathrm{H}_{36} \mathrm{NOSi}^{+}\left([\mathrm{M}+\mathrm{H}]^{+}\right) 358.2561$, found 358.2550

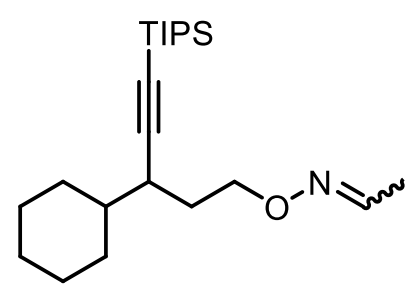

$3 q$

Following the general procedure, oxime 1q $(18.1 \mathrm{mg}, 0.100 \mathrm{mmol})$ was converted to the alkynylation product. Purification by column chromatography with EtOAc/Hexane as eluent gave the product 3q (19.6 mg, 54\% yield) as a colorless oil. Spectroscopic data are given as a mixture of cis and trans isomers (1:1). ${ }^{1} \mathbf{H}$ NMR (400 MHz, Chloroform- $d$ ) $\delta$ 7.40 (q, $J=5.8 \mathrm{~Hz}, 0.5 \mathrm{H}), 6.74$ (q, $J=5.5 \mathrm{~Hz}, 0.5 \mathrm{H}), 4.19(\mathrm{~m}, 2 \mathrm{H}), 2.42(\mathrm{~m}, 1 \mathrm{H}), 1.75(\mathrm{~m}$, 10H), $1.21(\mathrm{~m}, 6 \mathrm{H}), 1.06(\mathrm{~s}, 21 \mathrm{H}) .{ }^{13} \mathrm{C}$ NMR (101 MHz, Chloroform- $d$ ) $\delta$ 146.8, 146.6, $110.5,110.5,82.4,72.3,72.0,41.6,41.5,35.8,35.7,32.2,31.7,28.9,28.8,26.7,26.7,26.5$, 18.8, 15.4, 12.0, 11.5. IR $\vee\left(\mathrm{cm}^{-1}\right)$ 2925, 2863, 2360, 2341, 2164, 2030, 1463, 883, 676. HRMS (ESI-ion trap) calcd for $\mathrm{C}_{22} \mathrm{H}_{42} \mathrm{NOSi}^{+}\left([\mathrm{M}+\mathrm{H}]^{+}\right) 364.3030$, found 364.3015

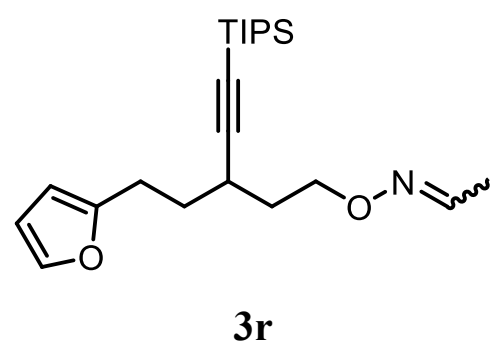


Following the general procedure, oxime $1 \mathbf{r}(19.3 \mathrm{mg}, 0.100 \mathrm{mmol})$ was converted to the alkynylation product. Purification by column chromatography with EtOAc/Hexane as eluent gave the product $3 \mathbf{r}$ (29.6 mg, 79\% yield) as a colorless oil. Spectroscopic data are given as a mixture of $c i s$ and trans isomers (1:1). ${ }^{1} \mathbf{H}$ NMR (400 MHz, Chloroform- $d$ ) $\delta$ $7.42(\mathrm{q}, J=5.8 \mathrm{~Hz}, 0.5 \mathrm{H}), 7.32(\mathrm{~d}, J=1.8 \mathrm{~Hz}, 1 \mathrm{H}), 6.76(\mathrm{q}, J=5.5 \mathrm{~Hz}, 0.5 \mathrm{H}), 6.30(\mathrm{~s}, 1 \mathrm{H})$, $6.02(\mathrm{~s}, 1 \mathrm{H}), 4.26(\mathrm{~m}, 2 \mathrm{H}), 2.90(\mathrm{~m}, 1 \mathrm{H}), 2.82(\mathrm{~m}, 1 \mathrm{H}), 2.60(\mathrm{~m}, 1 \mathrm{H}), 1.85(\mathrm{~m}, 7 \mathrm{H}), 1.10$ (s, 21H). ${ }^{13}$ C NMR (101 MHz, Chloroform- $d$ ) 155.9, 155.8, 146.9, 146.7, 141.0, 110.9, $110.9,110.2,105.1,105.1,82.4,82.3,71.7,71.4,34.6,33.8,33.7,29.2,26.0,18.8,15.4$, 12.0, 11.4. IR $v\left(\mathrm{~cm}^{-1}\right)$ 2942, 2864, 2360, 2341, 2165, 1463, 863, 661. HRMS (ESI-ion trap) calcd for $\mathrm{C}_{22} \mathrm{H}_{38} \mathrm{NO}_{2} \mathrm{Si}^{+}\left([\mathrm{M}+\mathrm{H}]^{+}\right) 376.2666$, found 376.2647

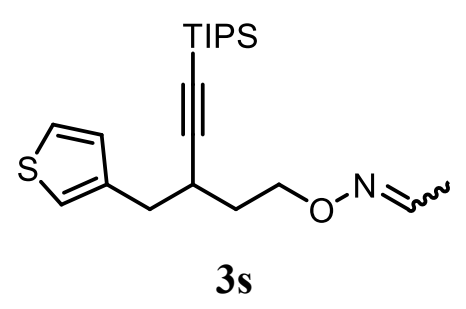

Following the general procedure, oxime $1 \mathrm{~s}(19.5 \mathrm{mg}, 0.100 \mathrm{mmol})$ was converted to the alkynylation product. Purification by column chromatography with EtOAc/Hexane as eluent gave the product $3 \mathrm{~s}$ ( $28.3 \mathrm{mg}, 75 \%$ yield) as a yellow oil. Spectroscopic data are given as a mixture of cis and trans isomers (1:1). ${ }^{1} \mathbf{H}$ NMR (400 MHz, Chloroform- $d$ ) $\delta 7.40$ (q, $J=5.8 \mathrm{~Hz}, 0.5 \mathrm{H}), 7.13(\mathrm{~m}, 1 \mathrm{H}), 6.90(\mathrm{~m}, 2 \mathrm{H}), 6.74(\mathrm{q}, J=5.5 \mathrm{~Hz}, 0.5 \mathrm{H}), 4.21(\mathrm{~m}, 2 \mathrm{H})$, $3.03(\mathrm{~m}, 2 \mathrm{H}), 2.86(\mathrm{~m}, 1 \mathrm{H}), 1.94(\mathrm{~m}, 1 \mathrm{H}), 1.84(\mathrm{~m}, 3 \mathrm{H}), 1.75(\mathrm{~m}, 1 \mathrm{H}), 1.08(\mathrm{~s}, 21 \mathrm{H}) .{ }^{13} \mathrm{C}$ NMR (101 MHz, Chloroform- $d$ ) $\delta$ 146.9, 146.8, 141.7, 141.7, 126.6, 126.6, 125.9, 125.9, $123.8,123.8,110.4,110.4,83.0,83.0,71.6,71.3,35.9,34.0,34.0,32.1,32.0,18.8,18.7$, 15.3, 12.0, 11.4. IR $v\left(\mathrm{~cm}^{-1}\right) 2941,2890,2864,2360,2341,2166,1462,1017,852$. HRMS (ESI-ion trap) calcd for $\mathrm{C}_{21} \mathrm{H}_{36} \mathrm{NOSSi}^{+}\left([\mathrm{M}+\mathrm{H}]^{+}\right) 378.2281$, found 378.2268

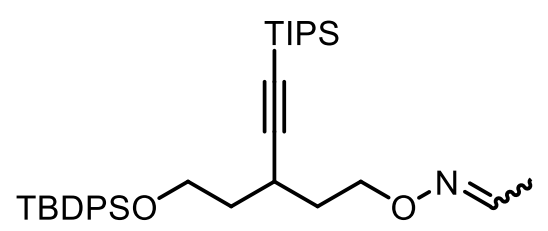

$3 t$ 
Following the general procedure, oxime 1t $(38.1 \mathrm{mg}, 0.100 \mathrm{mmol})$ was converted to the alkynylation product. Purification by column chromatography with EtOAc/Hexane as eluent gave the product $3 \mathbf{3}$ ( $41.7 \mathrm{mg}, 74 \%$ yield) as a colorless oil. Spectroscopic data are given as a mixture of cis and trans isomers (1:1). ${ }^{1} \mathbf{H}$ NMR (400 MHz, Chloroform- $d$ ) $\delta 7.69(\mathrm{~m}$, 4H), $7.41(\mathrm{~m}, 6.5 \mathrm{H}), 6.78(\mathrm{q}, J=5.5 \mathrm{~Hz}, 0.5 \mathrm{H}), 4.28(\mathrm{~m}, 2 \mathrm{H}), 3.90(\mathrm{~m}, 2 \mathrm{H}), 2.88(\mathrm{~m}, 1 \mathrm{H})$, $1.84(\mathrm{~m}, 7 \mathrm{H}), 1.07(\mathrm{~m}, 30 \mathrm{H}) .{ }^{13} \mathrm{C}$ NMR (101 MHz, Chloroform- $d$ ) $\delta$ 146.8, 146.6, 135.7, 135.6, 134.2, 134.1, 129.6, 127.7, 111.2, 81.7, 81.7, 71.7, 71.4, 62.0, 62.0, 38.3, 38.3, 34.8, $34.8,27.0,26.2,26.2,19.4,18.8,15.4,12.0,11.4$. IR $v\left(\mathrm{~cm}^{-1}\right)$ 2941, 2863, 2360, 2341, $2165,1463,1428,1110,701$. HRMS (ESI-ion trap) calcd for $\mathrm{C}_{34} \mathrm{H}_{54} \mathrm{NO}_{2} \mathrm{Si}_{2}{ }^{+}\left([\mathrm{M}+\mathrm{H}]^{+}\right)$ 564.3688 , found 564.3672

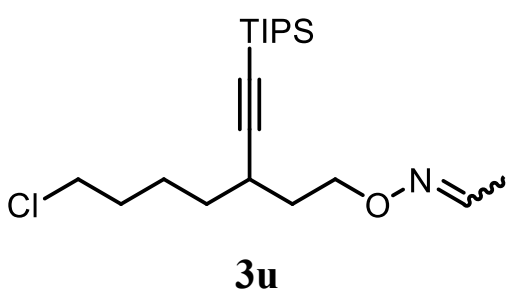

Following the general procedure, oxime $1 \mathbf{u}(18.9 \mathrm{mg}, 0.100 \mathrm{mmol})$ was converted to the alkynylation product. Purification by column chromatography with EtOAc/Hexane as eluent gave the product 3u (30.8 mg, 83\% yield) as a colorless oil. Spectroscopic data are given as a mixture of cis and trans isomers (1:1). ${ }^{1} \mathbf{H}$ NMR (400 MHz, Chloroform- $d$ ) $\delta$ 7.40 (q, $J=5.9 \mathrm{~Hz}, 0.5 \mathrm{H}), 6.75(\mathrm{q}, J=5.5 \mathrm{~Hz}, 0.5 \mathrm{H}), 4.23(\mathrm{~m}, 1 \mathrm{H}), 4.17(\mathrm{~m}, 1 \mathrm{H}), 3.53(\mathrm{~m}$, 2H), $2.54(\mathrm{~m}, 1 \mathrm{H}), 1.83(\mathrm{~m}, 8 \mathrm{H}), 1.50(\mathrm{~m}, 3 \mathrm{H}), 1.06(\mathrm{~m}, 21 \mathrm{H}) .{ }^{13} \mathrm{C}$ NMR $(101 \mathrm{MHz}$, Chloroform- $d$ ) $\delta 146.9,146.7,111.3,111.2,82.0,71.8,71.5,45.1,34.8,34.6,32.6,32.6,29.6$, 29.5, 24.8, 24.8, 18.8, 15.4, 12.0, 11.4. IR $v\left(\mathrm{~cm}^{-1}\right) 2943,2866,2360,2341,1717,1699$, 882, 669. HRMS (ESI-ion trap) calcd for $\mathrm{C}_{20} \mathrm{H}_{39} \mathrm{ClNOSi}^{+}\left([\mathrm{M}+\mathrm{H}]^{+}\right) 372.2484$, found 372.2468

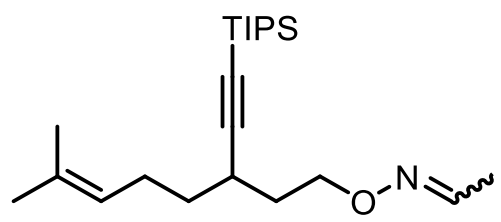

$3 v$ 
Following the general procedure, oxime $1 \mathbf{v}(18.1 \mathrm{mg}, 0.100 \mathrm{mmol})$ was converted to the alkynylation product. Purification by column chromatography with EtOAc/Hexane as eluent gave the product $3 \mathbf{v}$ ( $26.9 \mathrm{mg}, 74 \%$ yield) as a colorless oil. Spectroscopic data are given as a mixture of cis and trans isomers (1:1). ${ }^{1} \mathbf{H}$ NMR (400 MHz, Chloroform- $d$ ) $\delta$ 7.42 (q, $J=5.8 \mathrm{~Hz}, 0.5 \mathrm{H}), 6.76(\mathrm{q}, J=5.5 \mathrm{~Hz}, 0.5 \mathrm{H}), 5.13(\mathrm{~m}, 1 \mathrm{H}), 4.25(\mathrm{~m}, 2 \mathrm{H}), 2.55(\mathrm{~m}$, $1 \mathrm{H}), 2.22(\mathrm{~m}, 2 \mathrm{H}), 1.85(\mathrm{~m}, 5 \mathrm{H}), 1.71(\mathrm{~m}, 3 \mathrm{H}), 1.64(\mathrm{~m}, 3 \mathrm{H}), 1.50(\mathrm{~m}, 2 \mathrm{H}), 1.09(\mathrm{~s}, 21 \mathrm{H})$. ${ }^{13}$ C NMR (101 MHz, Chloroform- $d$ ) $\delta$ 146.8, 146.6, 132.2, 132.2, 124.1, 111.8, 111.7, 81.6, $81.6,71.9,71.6,35.7,35.6,34.8,29.3,29.3,26.0,25.9,18.8,17.8,15.4,12.0,11.4$. IR $v$ $\left(\mathrm{cm}^{-1}\right)$ 2941, 2864, 2360, 2341, 2164, 1462, 862, 660. HRMS (ESI-ion trap) calcd for $\mathrm{C}_{22} \mathrm{H}_{42} \mathrm{NOSi}^{+}\left([\mathrm{M}+\mathrm{H}]^{+}\right) 364.3030$, found 364.3015

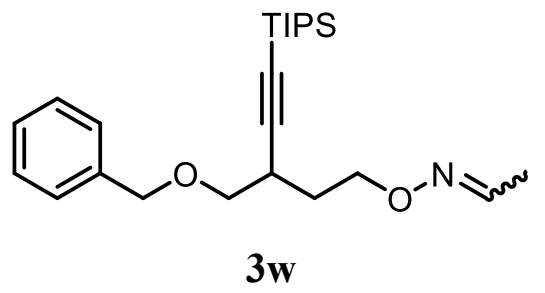

Following the general procedure, oxime $(Z)-1 \mathbf{w}(21.9 \mathrm{mg}, 0.100 \mathrm{mmol})$ was converted to the alkynylation product. Purification by column chromatography with EtOAc/Hexane as eluent gave the product $\mathbf{3 w}$ (9.20 $\mathrm{mg}, 23 \%$ yield) as a colorless oil.

Following the general procedure, oxime $(E)-\mathbf{1 w}(21.9 \mathrm{mg}, 0.100 \mathrm{mmol})$ was converted to the alkynylation product. Purification by column chromatography with EtOAc/Hexane as eluent gave the product $3 \mathbf{w}$ ( $27.7 \mathrm{mg}, 69 \%$ yield) as a colorless oil.

Spectroscopic data are given as a mixture of cis and trans isomers (1:1). ${ }^{1} \mathbf{H}$ NMR (400 MHz, Chloroform- $d$ ) $\delta 7.34(\mathrm{~m}, 5.5 \mathrm{H}), 6.74(\mathrm{~m}, 0.5 \mathrm{H}), 4.57(\mathrm{~m}, 2 \mathrm{H}), 4.27(\mathrm{~m}, 2 \mathrm{H}), 3.61$ (m, 1H), $3.50(\mathrm{~m}, 1 \mathrm{H}), 2.89(\mathrm{~m}, 1 \mathrm{H}), 2.07(\mathrm{~m}, 1 \mathrm{H}), 1.84(\mathrm{~m}, 3 \mathrm{H}), 1.75(\mathrm{~m}, 1 \mathrm{H}), 1.03(\mathrm{~m}$, 21H). ${ }^{13}$ C NMR (101 MHz, Chloroform- $d$ ) $\delta$ 146.9, 146.7, 138.5, 128.5, 127.9, 127.6, 109.0, 82.5, 73.4, 73.3, 73.2, 73.2, 72.4, 71.5, 71.2, 31.5, 31.5, 30.7, 30.6, 18.8, 15.4, 12.0, 11.4, 11.4. IR $v\left(\mathrm{~cm}^{-1}\right)$ 2941, 2864, 2360, 2341, 2167, 1463, 860. HRMS (ESI-ion trap) calcd for $\mathrm{C}_{24} \mathrm{H}_{40} \mathrm{NO}_{2} \mathrm{Si}^{+}\left([\mathrm{M}+\mathrm{H}]^{+}\right) 402.2823$, found 402.2805 


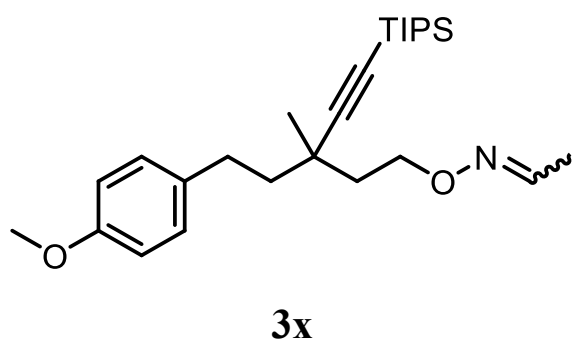

Following the general procedure, oxime 1x $(24.7 \mathrm{mg}, 0.100 \mathrm{mmol})$ was converted to the alkynylation product. Purification by column chromatography with EtOAc/Hexane as eluent gave the product 3x (16.3 mg, 38\% yield) as a colorless oil. Spectroscopic data are given as a mixture of cis and trans isomers (1:1). ${ }^{1} \mathbf{H}$ NMR (400 MHz, Chloroform- $d$ ) $\delta$ 7.39 (q, $J=5.8 \mathrm{~Hz}, 0.5 \mathrm{H}), 7.10(\mathrm{~m}, 2 \mathrm{H}), 6.83(\mathrm{~m}, 2 \mathrm{H}), 6.74$ (q, $J=5.5 \mathrm{~Hz}, 0.5 \mathrm{H}), 4.29$ (m, 2H), $3.79(\mathrm{~s}, 3 \mathrm{H}), 2.75(\mathrm{~m}, 2 \mathrm{H}), 1.90(\mathrm{~m}, 1 \mathrm{H}), 1.83(\mathrm{~m}, 5 \mathrm{H}), 1.65(\mathrm{~m}, 1 \mathrm{H}), 1.28(\mathrm{~m}, 3 \mathrm{H})$, $1.08(\mathrm{~s}, 21 \mathrm{H}) .{ }^{13} \mathrm{C}$ NMR (101 MHz, Chloroform- $d$ ) $\delta$ 146.9, 146.7, 138.5, 128.5, 127.9, 127.6, 109.0, 82.5, 73.4, 73.3, 73.2, 73.2, 72.4, 71.5, 71.2, 31.5, 31.5, 30.7, 30.6, 18.8, 15.4, 12.0, 11.4, 11.4. IR $v\left(\mathrm{~cm}^{-1}\right) 2931,2858,2361,2341,1511,1245,1035,824$. HRMS (ESIion trap) calcd for $\mathrm{C}_{26} \mathrm{H}_{44} \mathrm{NO}_{2} \mathrm{Si}^{+}\left([\mathrm{M}+\mathrm{H}]^{+}\right) 430.3136$, found 430.3118

\section{Synthetic transformation of product}

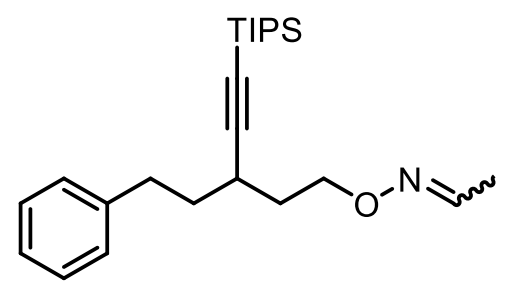

$3 a$

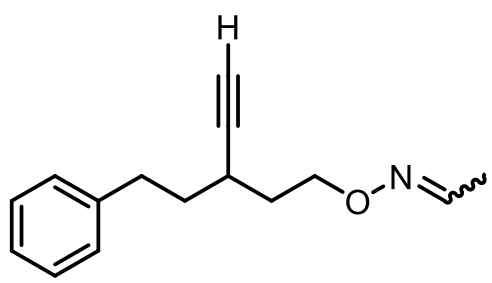

$4 a$

To a solution of 3a (20 mg, $0.050 \mathrm{mmol})$ and $0.50 \mathrm{~mL}$ THF was added TBAF (1.0 M in THF, $0.50 \mathrm{~mL}$ ). The reaction was stirred at room temperature for $5 \mathrm{~min}$. Then the crude mixture was purified by column chromatography with EtOAc/Hexane as eluent to give 4a (20 mg, 86\%) as a colorless oil. Spectroscopic data are given as a mixture of cis and trans isomers (1:1). ${ }^{1} \mathbf{H}$ NMR (400 MHz, Chloroform- $d$ ) $\delta 7.38(\mathrm{q}, J=5.8 \mathrm{~Hz}, 0.5 \mathrm{H}), 7.28(\mathrm{~m}$, 2H), $7.22(\mathrm{~m}, 3 \mathrm{H}), 6.73(\mathrm{q}, J=5.5 \mathrm{~Hz}, 0.5 \mathrm{H}), 4.23(\mathrm{~m}, 2 \mathrm{H}), 2.75(\mathrm{~m}, 1 \mathrm{H}), 2.73(\mathrm{~m}, 1 \mathrm{H})$, 
$2.51(\mathrm{~m}, 1 \mathrm{H}), 2.14(\mathrm{~s}, 1 \mathrm{H}), 1.81(\mathrm{~m}, 7 \mathrm{H}) .{ }^{13} \mathbf{C}$ NMR (101 MHz, Chloroform- $d$ ) $\delta$ 147.0, $146.9,142.0,142.0,128.6,128.5,128.5,126.0,86.9,86.8,71.5,71.2,70.4,70.3,36.9$, $36.8,34.4,34.3,33.6,33.5,27.9,27.9,15.4,12.0$. IR $v\left(\mathrm{~cm}^{-1}\right) 3294,2925,2361,2341$, 1053, 700. HRMS (ESI-ion trap) calcd for $\mathrm{C}_{15} \mathrm{H}_{20} \mathrm{NO}^{+}\left([\mathrm{M}+\mathrm{H}]^{+}\right)$230.1539, found 230.1532

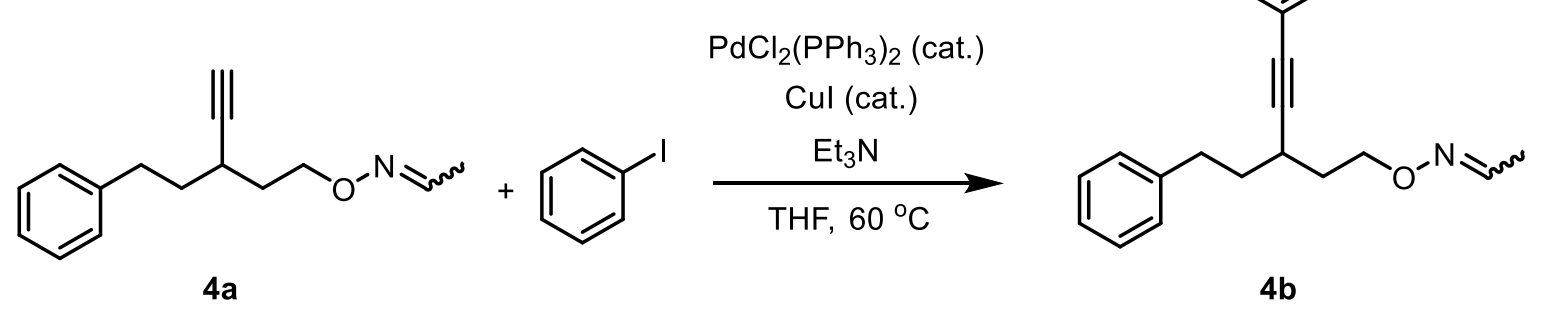

To a Schlenk flask was added $4 \mathbf{a}(10 \mathrm{mg}, 0.044 \mathrm{mmol}), \mathrm{PdCl}_{2}\left(\mathrm{PPh}_{3}\right)_{2}(1.5 \mathrm{mg}, 5.0 \mathrm{~mol} \%)$, $\mathrm{CuI}(0.50 \mathrm{mg}, 5.0 \mathrm{~mol} \%), \mathrm{Et}_{3} \mathrm{~N}$ (13 mg, 3.0 equiv) and $2.0 \mathrm{~mL}$ THF. The reaction was stirred at $60{ }^{\circ} \mathrm{C}$ for $2 \mathrm{~h}$. The mixture was purified by column chromatography with EtOAc/Hexane as eluent to give $\mathbf{4 b}(9.8 \mathrm{mg}, 73 \%)$ as a colorless oil. Spectroscopic data are given as a mixture of cis and trans isomers (1:1). ${ }^{1} \mathbf{H}$ NMR (400 MHz, Chloroform- $d$ ) $\delta 7.43(\mathrm{~m}, 2.5 \mathrm{H}), 7.27(\mathrm{~m}, 8 \mathrm{H}), 6.74(\mathrm{q}, J=5.5 \mathrm{~Hz}, 1 \mathrm{H}), 4.28(\mathrm{~m}, 2 \mathrm{H}), 2.93(\mathrm{~m}, 1 \mathrm{H}), 2.72$ (m, 2H), $1.82(\mathrm{~m}, 7 \mathrm{H}) .{ }^{13} \mathbf{C}$ NMR (101 MHz, Chloroform- $d$ ) $\delta$ 147.0, 146.9, 142.1, 142.1, $131.8,128.7,128.5,128.3,127.8,126.0,124.0,92.4,92.3,82.9,82.8,71.8,71.5,37.1$, 37.1, 34.6, 34.6, 33.8, 33.8, 29.9, 28.8, 28.8, 15.4, 12.0. IR $v\left(\mathrm{~cm}^{-1}\right)$ 3027, 2923, 2857, 1727, 756, 693. HRMS (ESI-ion trap) calcd for $\mathrm{C}_{21} \mathrm{H}_{24} \mathrm{NO}^{+}\left([\mathrm{M}+\mathrm{H}]^{+}\right) 306.1852$, found 306.1842

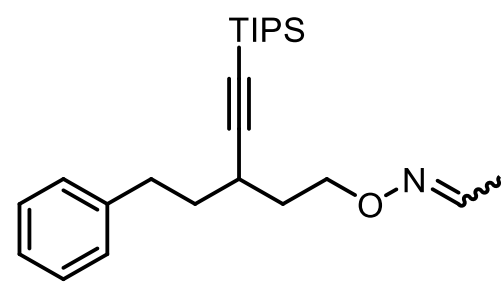

$3 a$

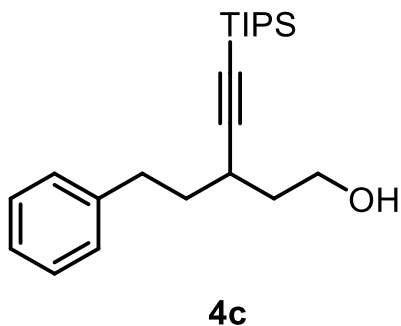

4c

To a solution of $\mathrm{LiAlH}_{4}(7.8 \mathrm{mg}, 0.20 \mathrm{mmol})$ and $1.0 \mathrm{~mL}$ THF was added $\mathbf{3 a}(20 \mathrm{mg}, 0.050$ $\mathrm{mmol}$ ). The reaction was stirred at room temperature for $1 \mathrm{~h}$. The mixture was purified by 
column chromatography with EtOAc/Hexane as eluent to give $\mathbf{4 c}(15 \mathrm{mg}, 81 \%)$ as a colorless yield. ${ }^{1} \mathbf{H}$ NMR (400 MHz, Chloroform- $d$ ) $\delta 7.27$ (m, 2H), 7.20 (m, 3H), 3.84 (t, $J=$ $5.5 \mathrm{~Hz}, 2 \mathrm{H}), 2.89(\mathrm{~m}, 1 \mathrm{H}), 2.75(\mathrm{~m}, 1 \mathrm{H}), 2.57(\mathrm{~m}, 1 \mathrm{H}), 1.77(\mathrm{~m}, 5 \mathrm{H}), 1.10(\mathrm{~s}, 21 \mathrm{H}) .{ }^{13} \mathrm{C}$ NMR (101 MHz, Chloroform-d) $\delta 142.1,128.7,128.5,126.0,111.5,83.1,61.6,38.1,37.6$, 33.7, 29.6, 18.8, 11.4. HRMS (ESI-ion trap) calcd for $\mathrm{C}_{22} \mathrm{H}_{37} \mathrm{OSi}^{+}\left([\mathrm{M}+\mathrm{H}]^{+}\right) 345.2608$, found 345.2598

\section{Ee determination of $\mathbf{3 a}$ and $\mathbf{3 d}$}
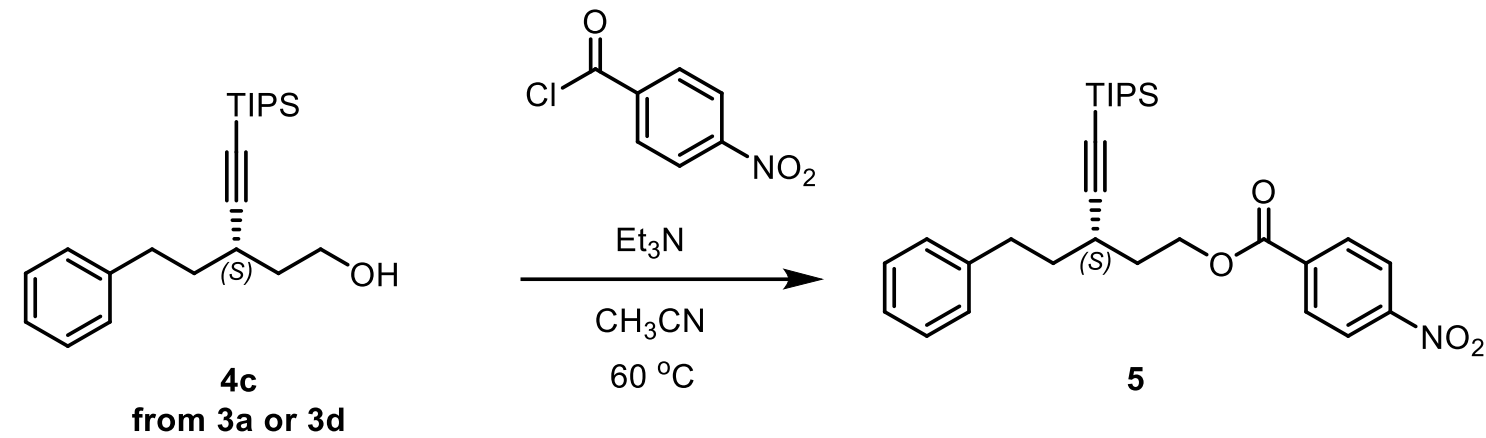

from $3 a$ or $3 d$

The ee value of 3a and 3d were determined by chiral HPLC analysis of the $p$-nitrobenzoate derivative following a literature procedure. ${ }^{2}$

Ee of 3a $(22 \%)$ was determined by CHIRALCEL® OD-H, $4.6 \mathrm{~mm} \times 250 \mathrm{~mm}$, hexane/2propanol $=99: 1,0.5 \mathrm{~mL} / \mathrm{min}, 40{ }^{\circ} \mathrm{C}$, retention time $=22.3 \mathrm{~min}$ for $R$ isomer and $30.4 \mathrm{~min}$ for $S$ isomer. 


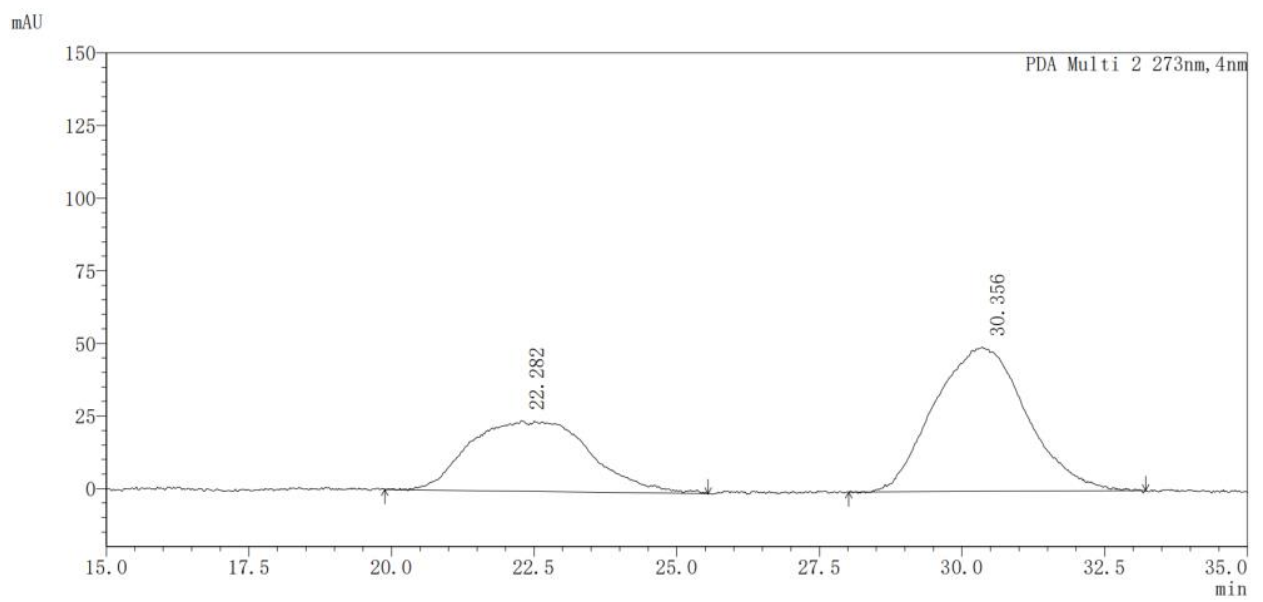

PDA Ch2 273nm

\begin{tabular}{|r|r|r|r|r|}
\hline Peak & Ret. Time & Height & \multicolumn{1}{l|}{ Area } & \multicolumn{1}{c|}{ Area\% } \\
\hline 1 & 22.282 & 24364 & 3571922 & 39.023 \\
\hline 2 & 30.356 & 49659 & 5581557 & 60.977 \\
\hline
\end{tabular}

Ee of 3d (47\%) was determined by CHIRALCEL ${ }^{\circledR}$ OD-H, $4.6 \mathrm{~mm} \times 250 \mathrm{~mm}$, hexane/2propanol $=99: 1,0.5 \mathrm{~mL} / \mathrm{min}, 40{ }^{\circ} \mathrm{C}$, retention time $=22.7 \mathrm{~min}$ for $R$ isomer and $29.8 \mathrm{~min}$ for $S$ isomer.

mAU

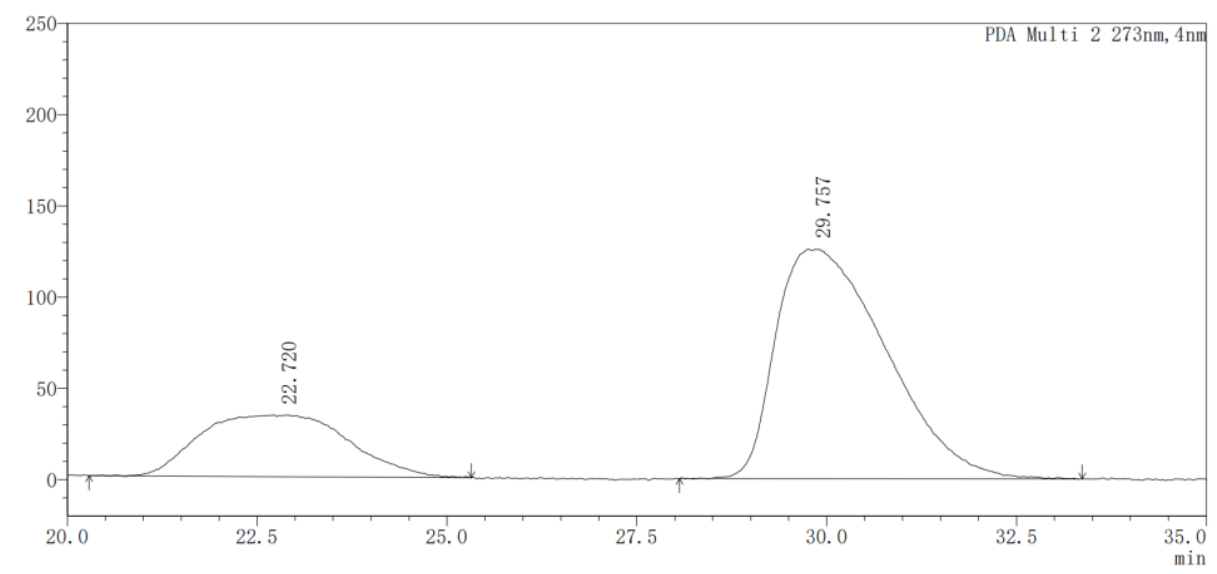

PDA Ch2 273nm

\begin{tabular}{|r|r|r|r|r|}
\hline Peak & Ret. Time & \multicolumn{1}{c|}{ Height } & \multicolumn{1}{c|}{ Area } & \multicolumn{1}{c|}{ Area\% } \\
\hline 1 & 22.720 & 33932 & 4600181 & 26.636 \\
\hline 2 & 29.757 & 125804 & 12670217 & 73.364 \\
\hline
\end{tabular}




\section{References}

1. Tsuchikama, K.; Kasagawa, M.; Endo, K.; Shibata, T. Cationic $\operatorname{Ir}(\mathrm{I})$-Catalyzed $\mathrm{sp}^{3} \mathrm{C}-\mathrm{H}$ Bond Alkenylation of Amides with Alkynes. Org. Lett. 2009, 11, 1821-1823.

2. Harada, A.; Makida, Y.; Sato, T.; Ohmiya, H.; Sawamura, M. Copper-Catalyzed Enantioselective Allylic Alkylation of Terminal Alkyne Pronucleophiles. J. Am. Chem. Soc. 2014, 136, 13932-13939. 


\section{Copy of NMR spectra}

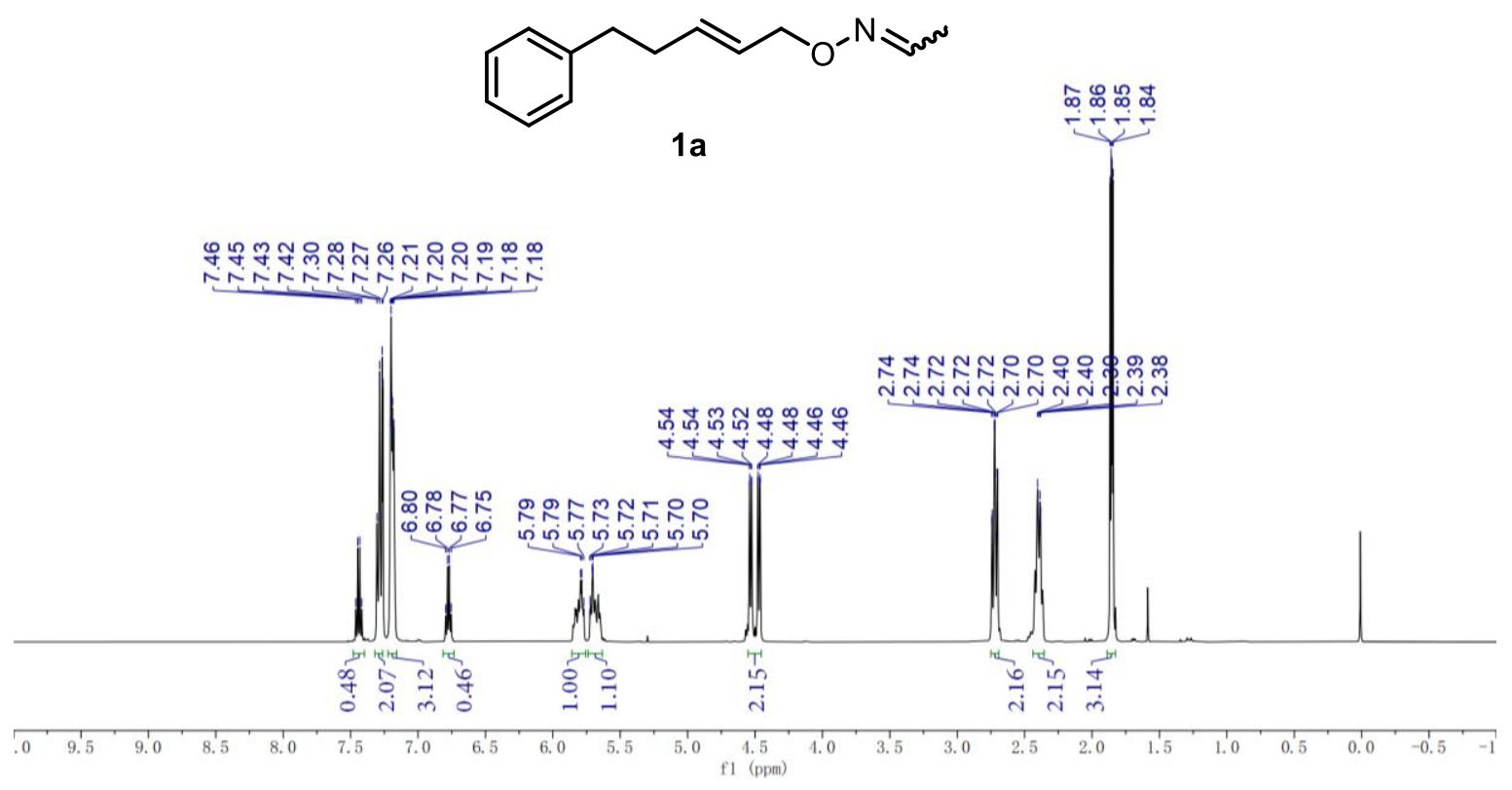

${ }^{1} \mathrm{H}$ NMR spectrum of $\mathbf{1 a}$ was measured in $\mathrm{CDCl}_{3}$ at $400 \mathrm{MHz}$
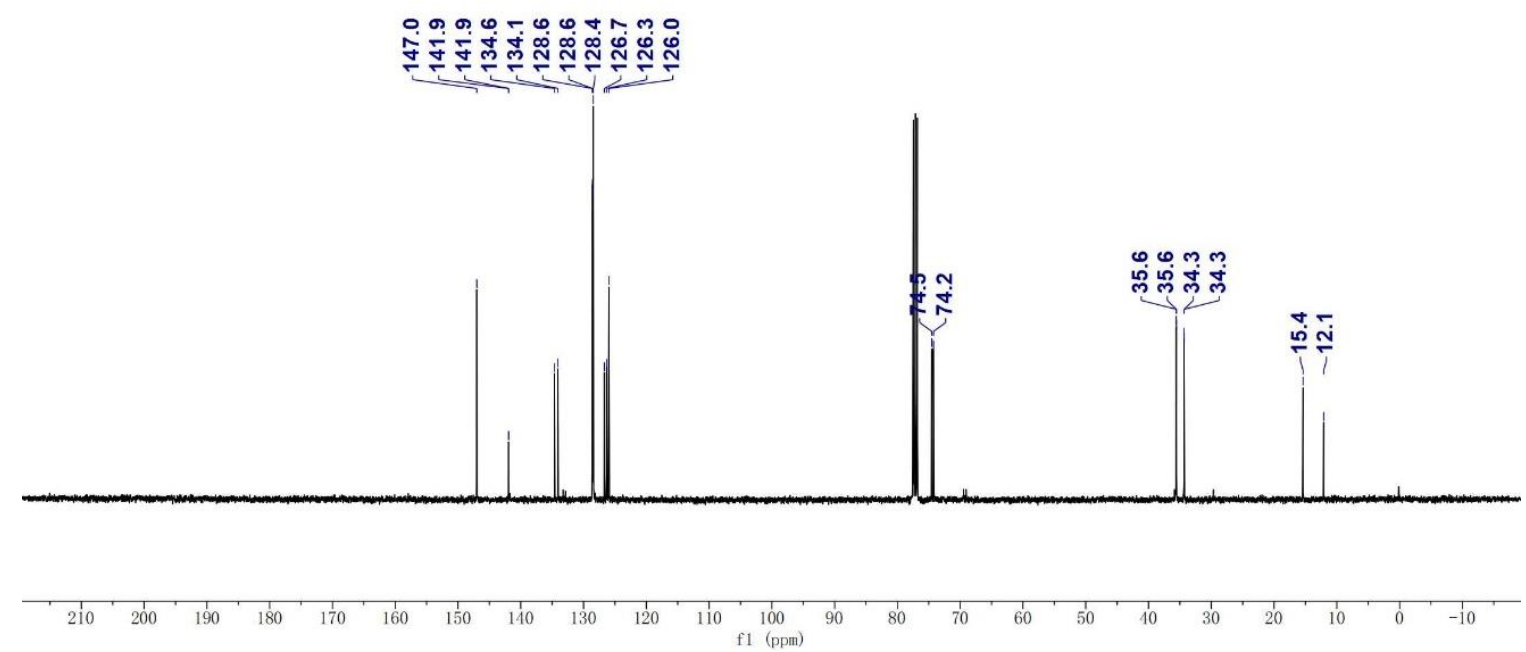

${ }^{13} \mathrm{C}$ NMR spectrum of $1 \mathrm{a}$ was measured in $\mathrm{CDCl}_{3}$ at $101 \mathrm{MHz}$ 


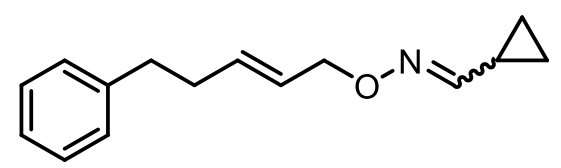

$1 \mathrm{~b}$

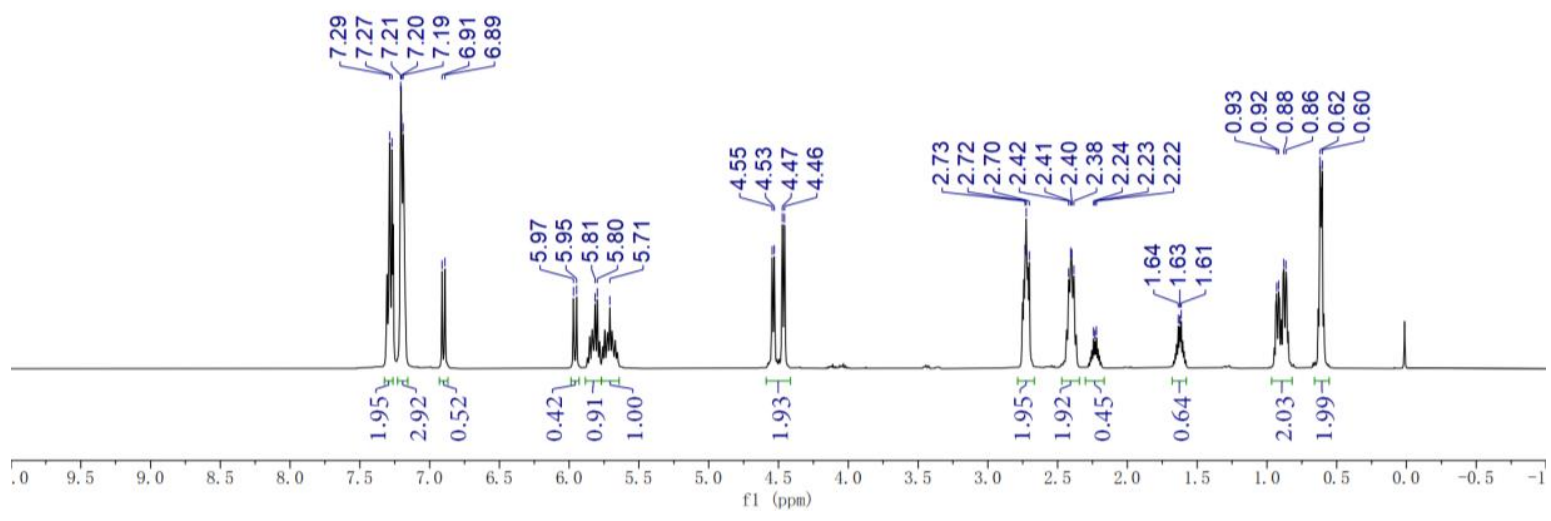

${ }^{1} \mathrm{H} \mathrm{NMR}$ spectrum of $\mathbf{1 b}$ was measured in $\mathrm{CDCl}_{3}$ at $400 \mathrm{MHz}$
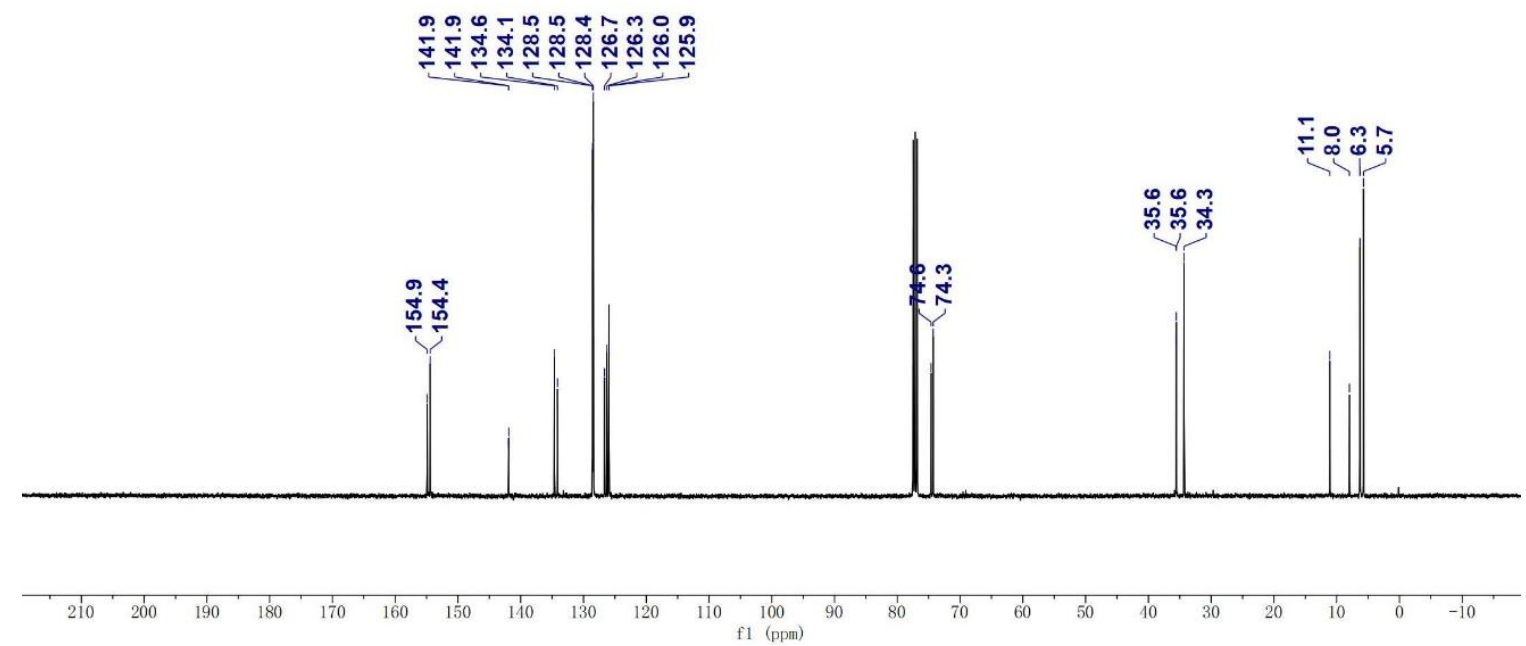

${ }^{13} \mathrm{C}$ NMR spectrum of $\mathbf{1 b}$ was measured in $\mathrm{CDCl}_{3}$ at $101 \mathrm{MHz}$ 


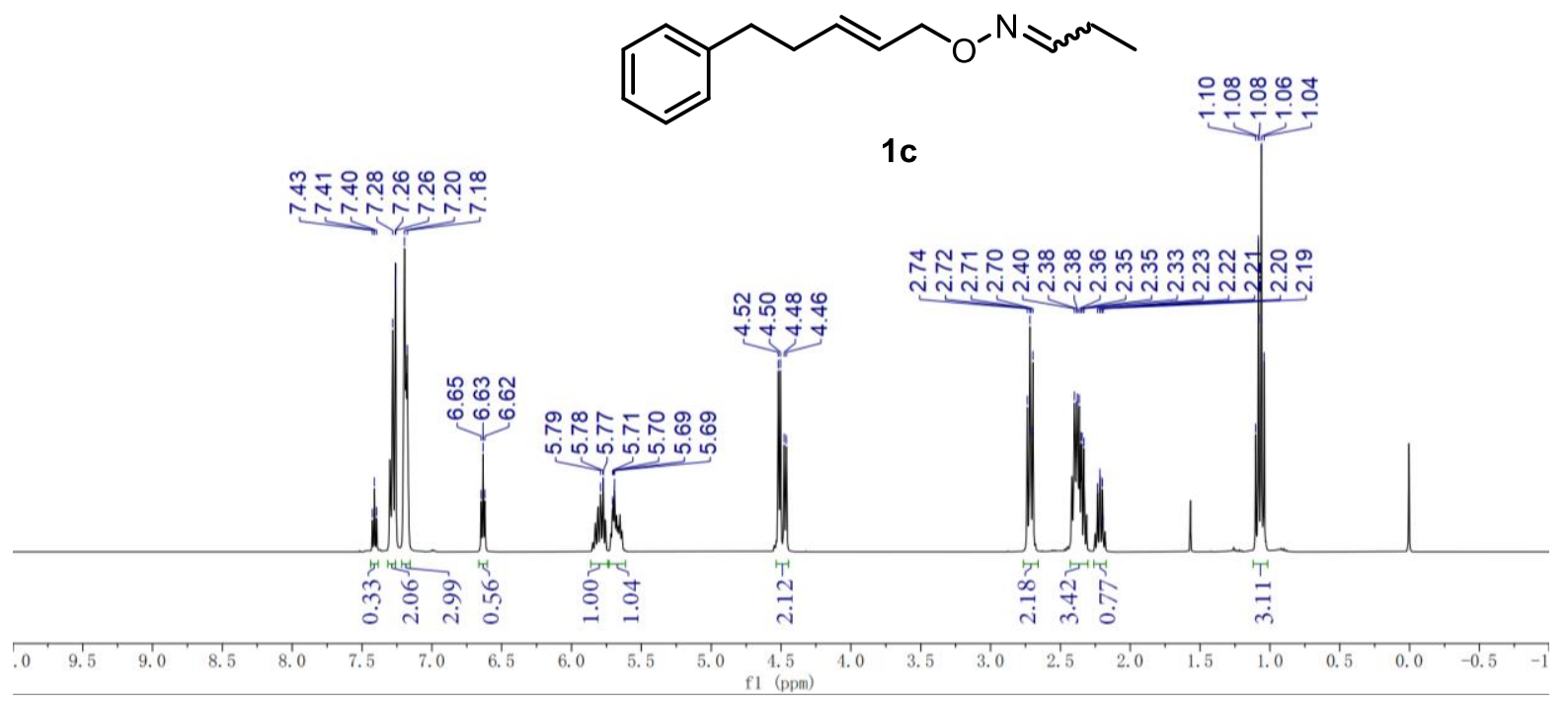

${ }^{1} \mathrm{H}$ NMR spectrum of $1 \mathrm{c}$ was measured in $\mathrm{CDCl}_{3}$ at $400 \mathrm{MHz}$
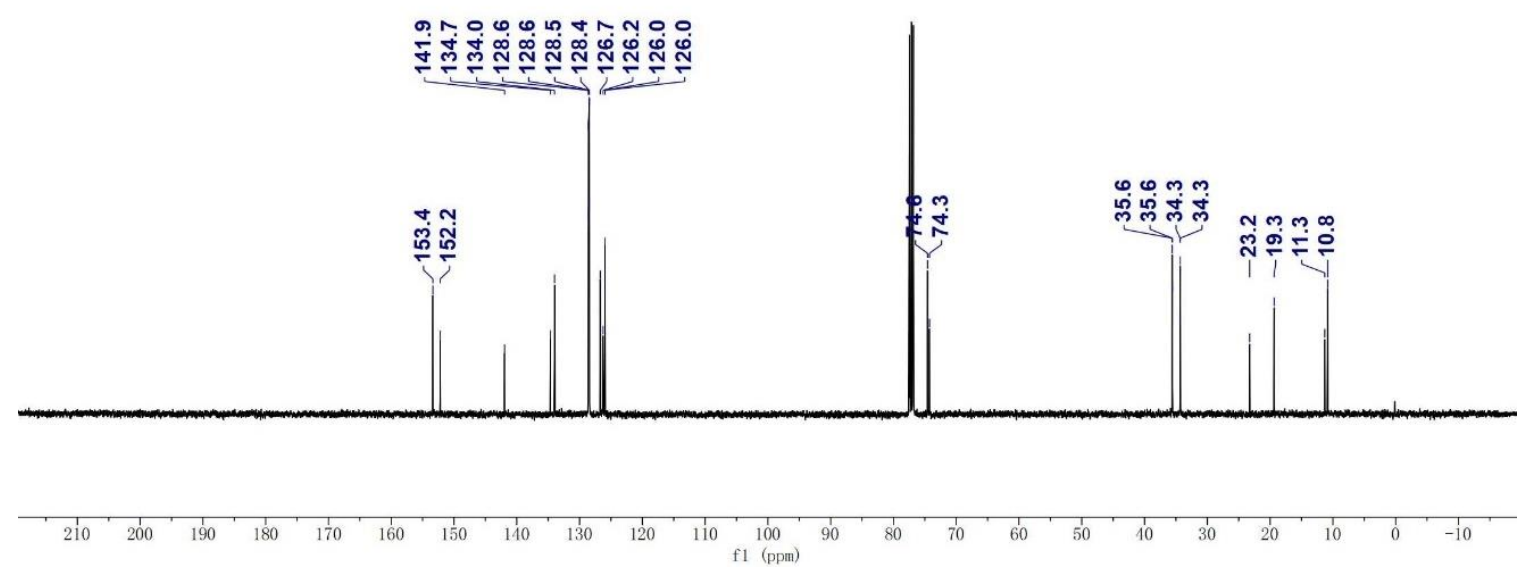

${ }^{13} \mathrm{C}$ NMR spectrum of $1 \mathrm{c}$ was measured in $\mathrm{CDCl}_{3}$ at $101 \mathrm{MHz}$ 


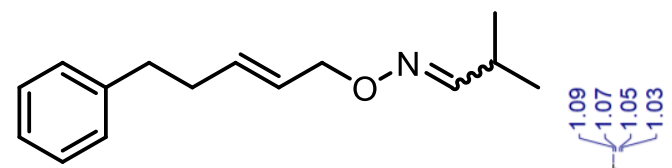

1d

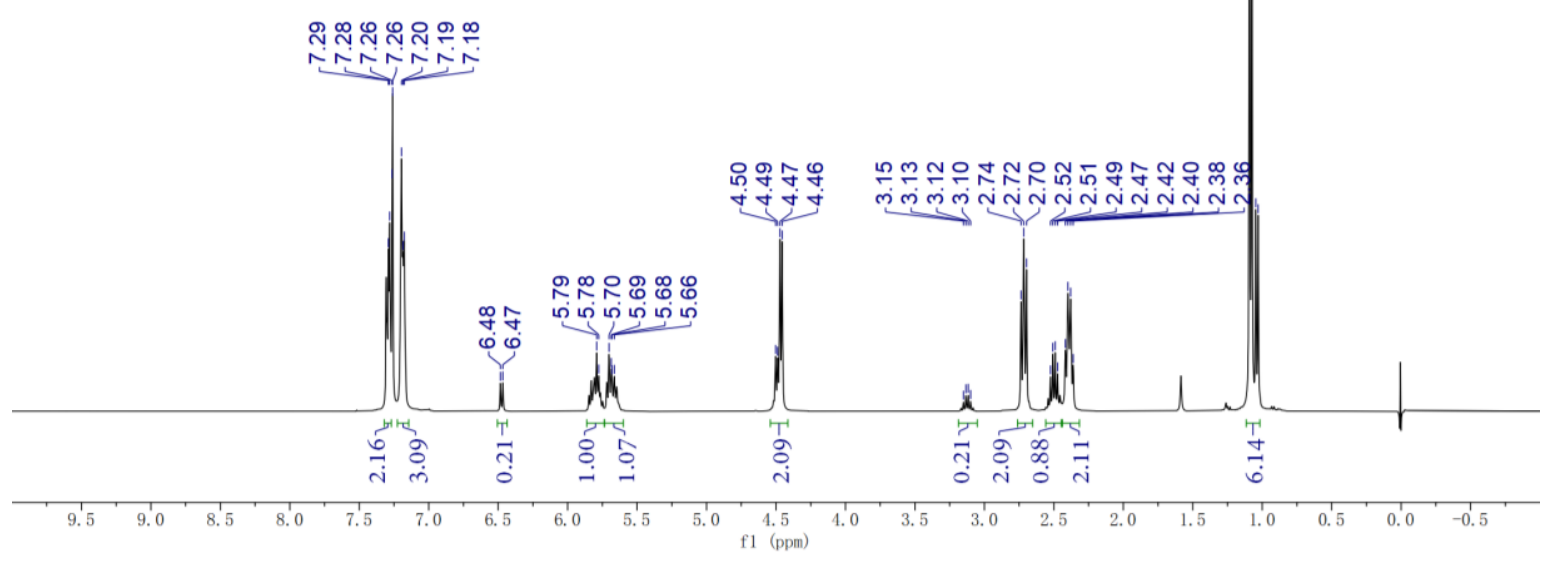

${ }^{1} \mathrm{H}$ NMR spectrum of $\mathbf{1 d}$ was measured in $\mathrm{CDCl}_{3}$ at $400 \mathrm{MHz}$
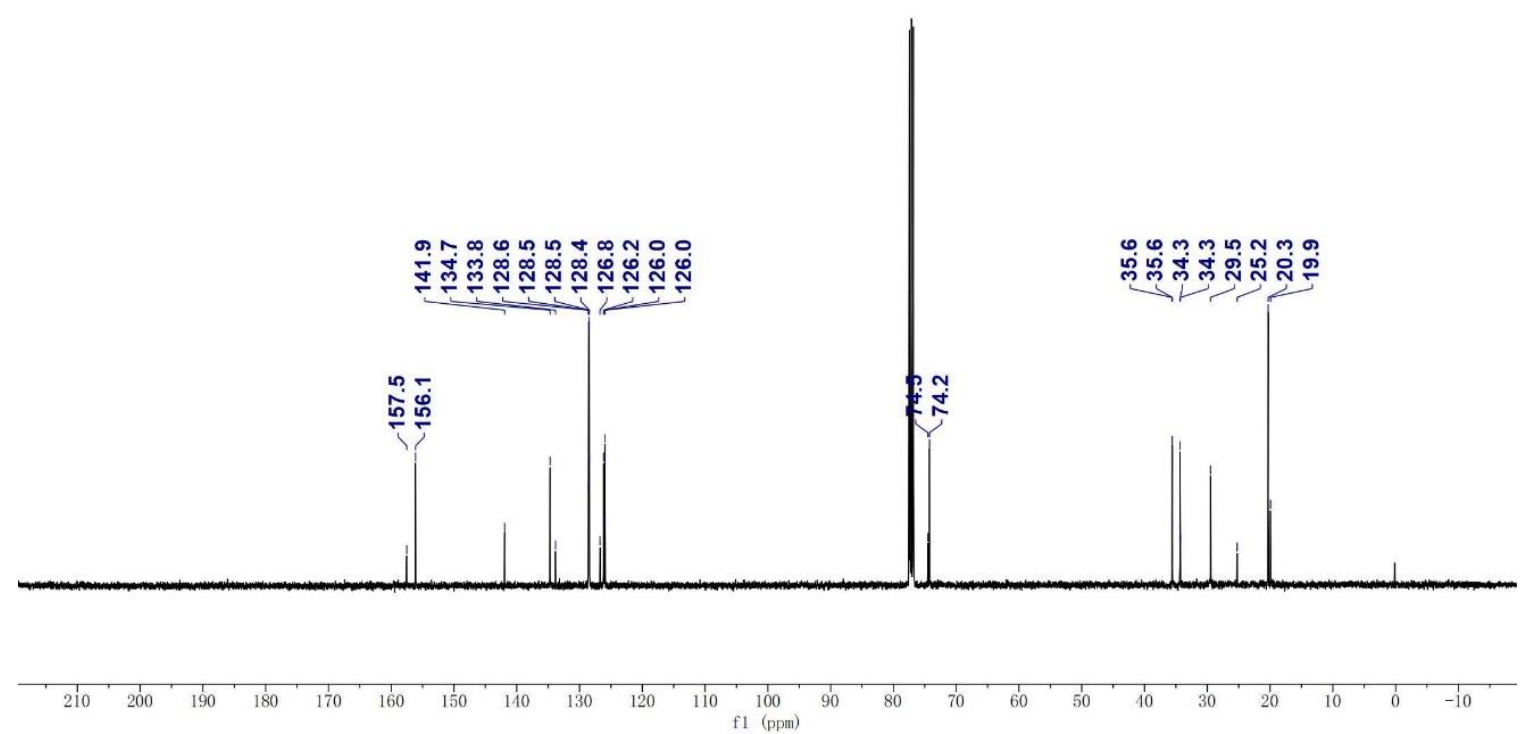

${ }^{13} \mathrm{C}$ NMR spectrum of $\mathbf{1 d}$ was measured in $\mathrm{CDCl}_{3}$ at $101 \mathrm{MHz}$ 

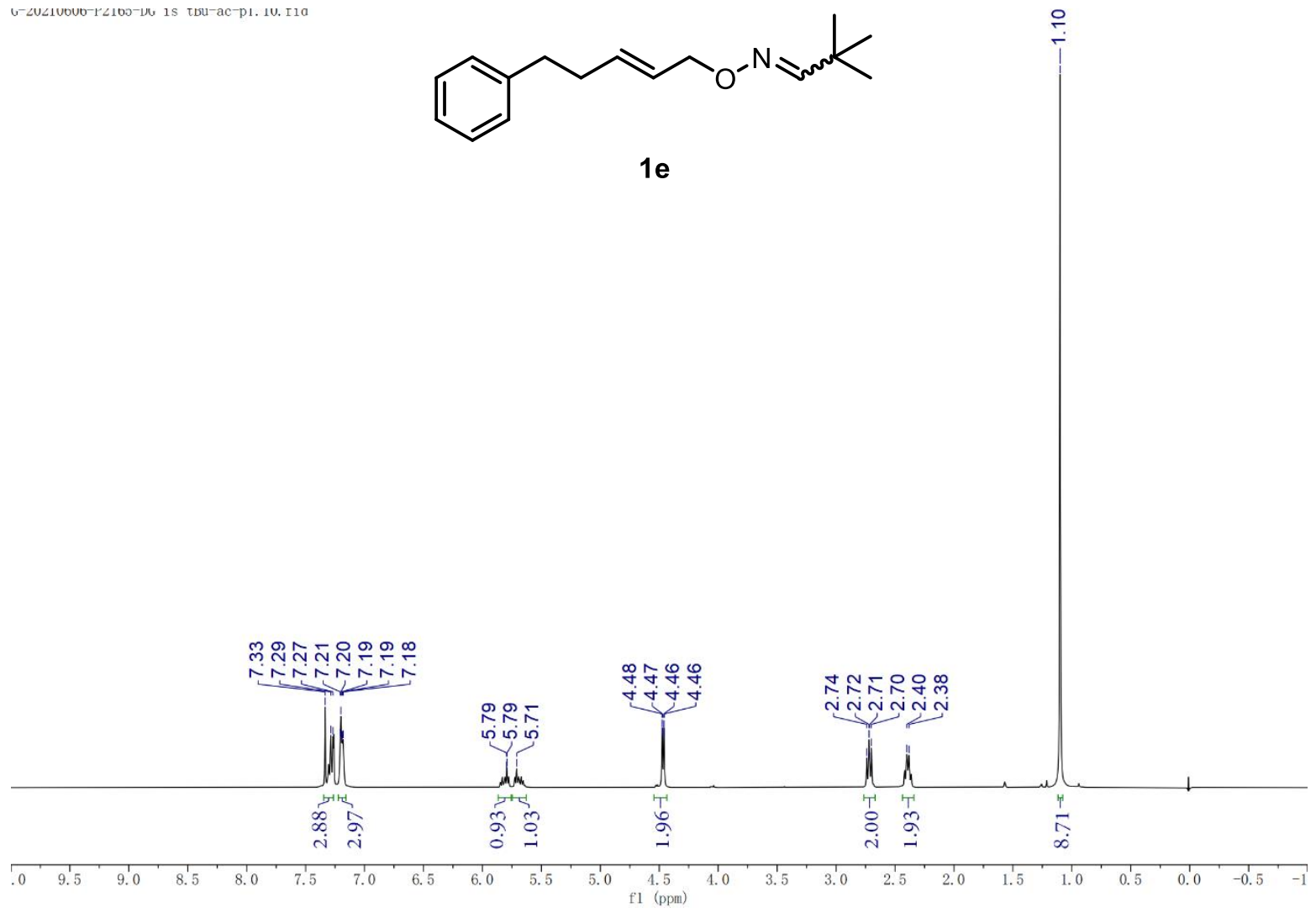

${ }^{1} \mathrm{H}$ NMR spectrum of 1 e was measured in $\mathrm{CDCl}_{3}$ at $400 \mathrm{MHz}$
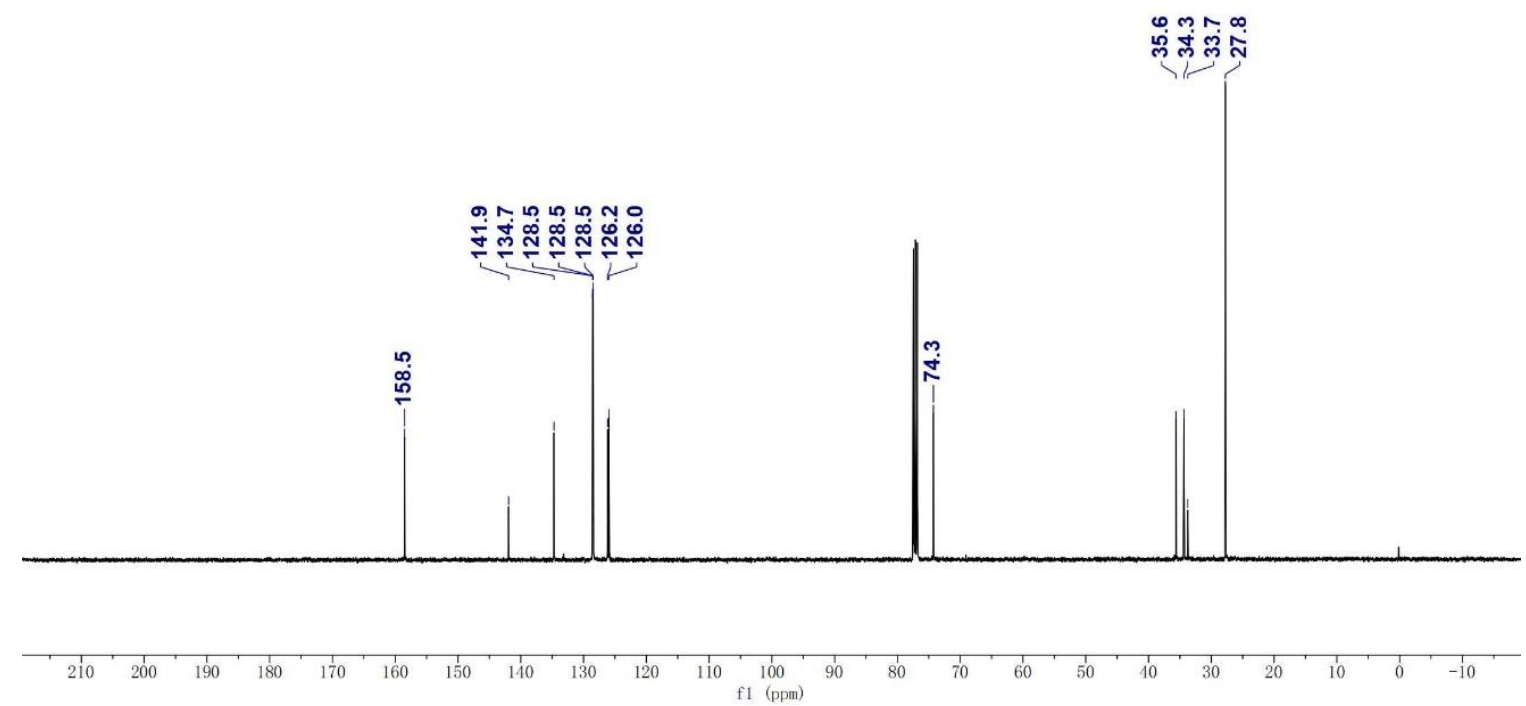

${ }^{13} \mathrm{C}$ NMR spectrum of 1 e was measured in $\mathrm{CDCl}_{3}$ at $101 \mathrm{MHz}$ 


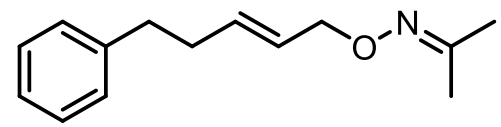

$1 f$

\section{$\stackrel{\substack{\infty \\ \infty}}{\longrightarrow \infty}$}

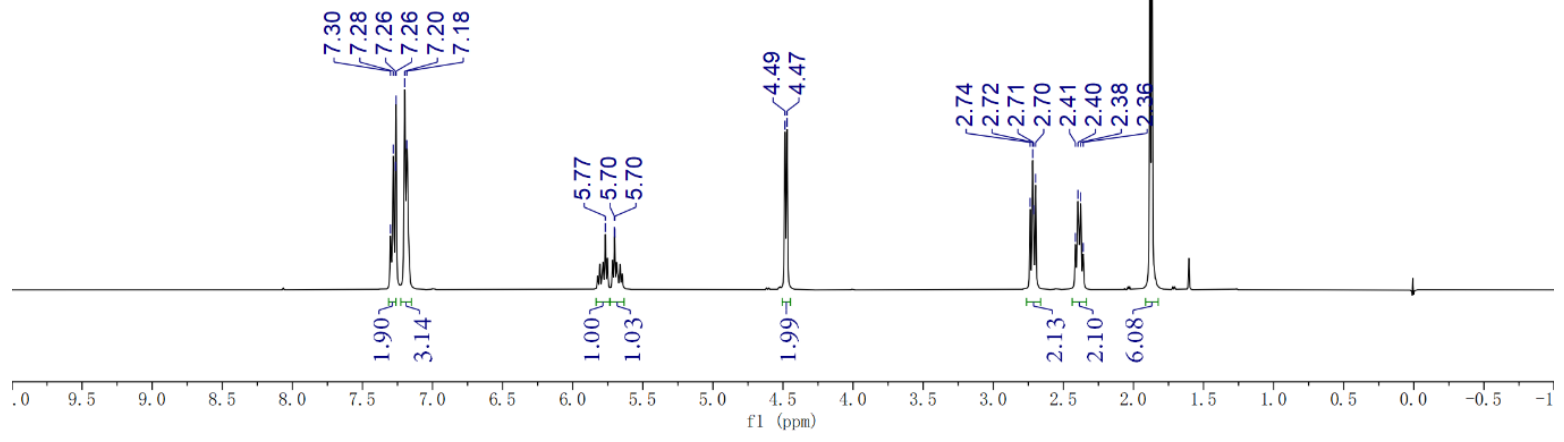

${ }^{1} \mathrm{H}$ NMR spectrum of $\mathbf{1 f}$ was measured in $\mathrm{CDCl}_{3}$ at $400 \mathrm{MHz}$
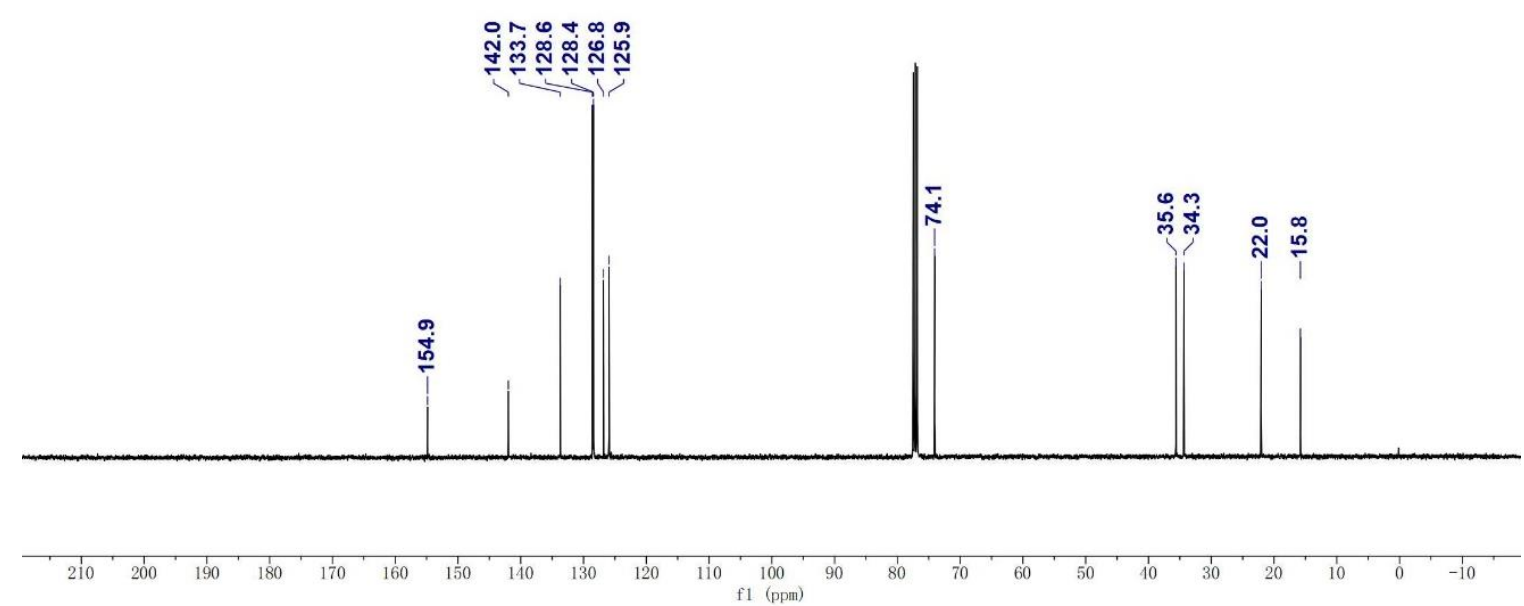

${ }^{13} \mathrm{C}$ NMR spectrum of $\mathbf{1 f}$ was measured in $\mathrm{CDCl}_{3}$ at $101 \mathrm{MHz}$ 


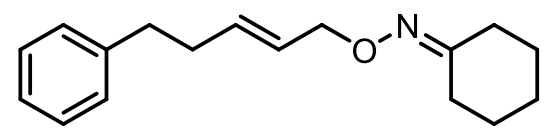

$1 \mathrm{~g}$

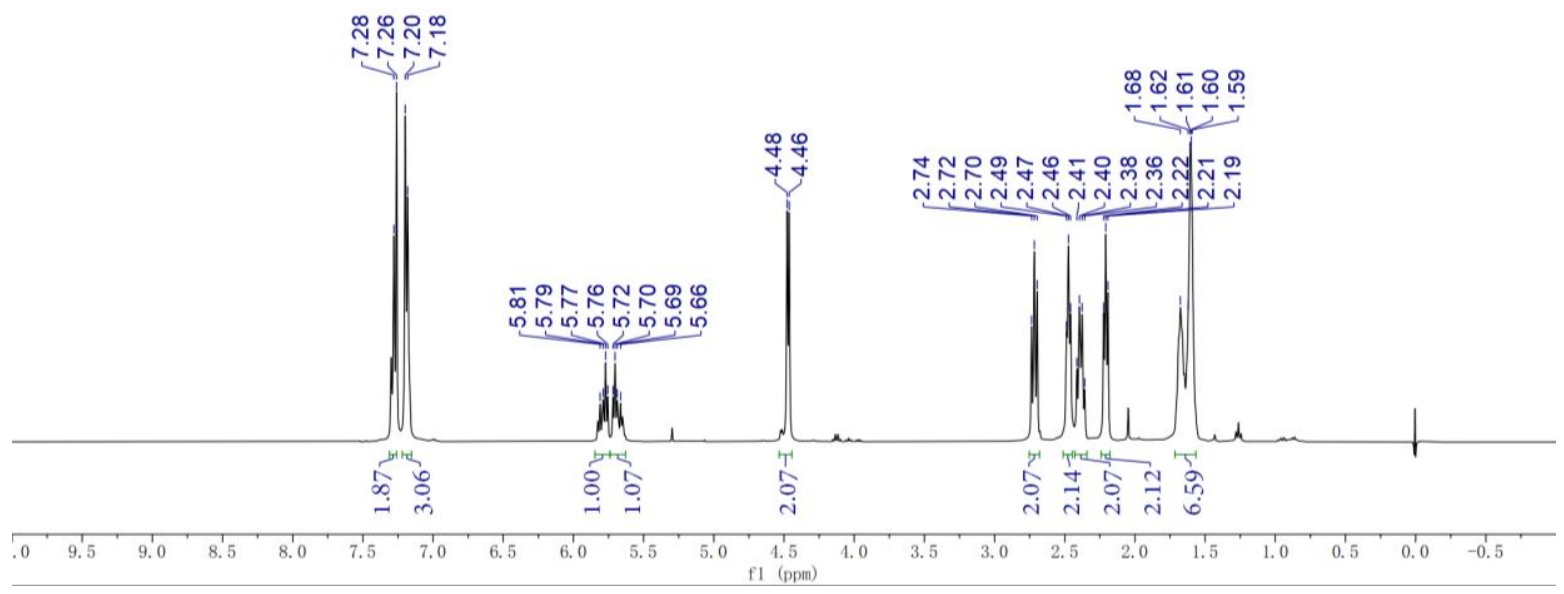

${ }^{1} \mathrm{H}$ NMR spectrum of $\mathbf{1 g}$ was measured in $\mathrm{CDCl}_{3}$ at $400 \mathrm{MHz}$
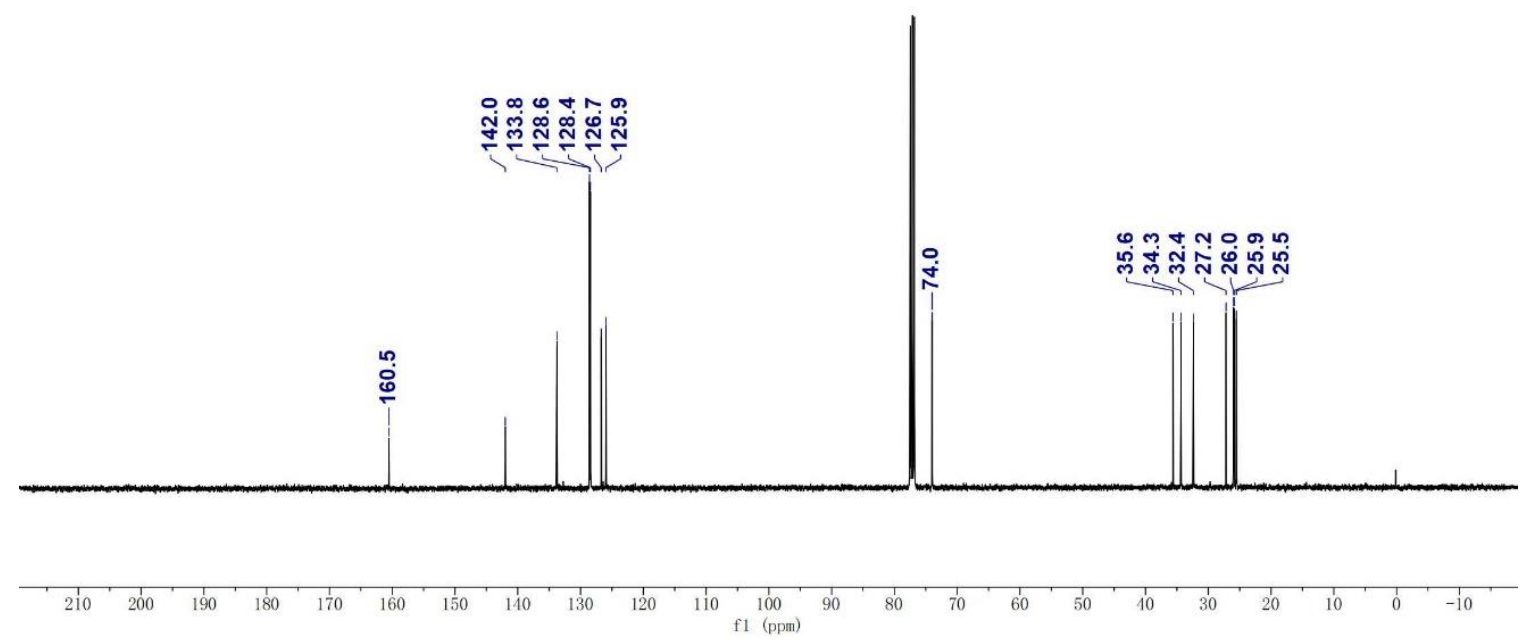

${ }^{13} \mathrm{C}$ NMR spectrum of $\mathbf{1 g}$ was measured in $\mathrm{CDCl}_{3}$ at $101 \mathrm{MHz}$ 


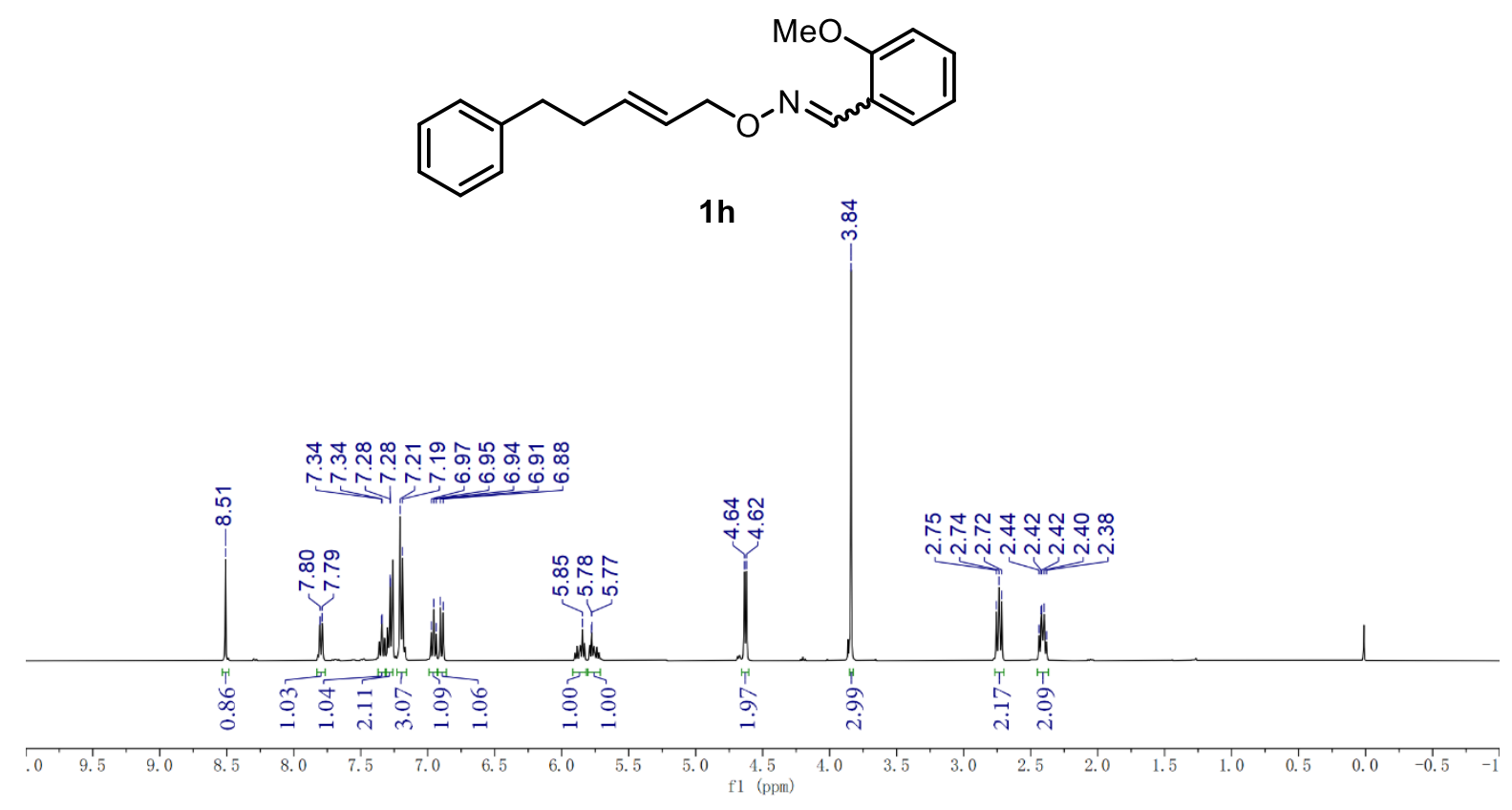

${ }^{1} \mathrm{H}$ NMR spectrum of $\mathbf{1 h}$ was measured in $\mathrm{CDCl}_{3}$ at $400 \mathrm{MHz}$
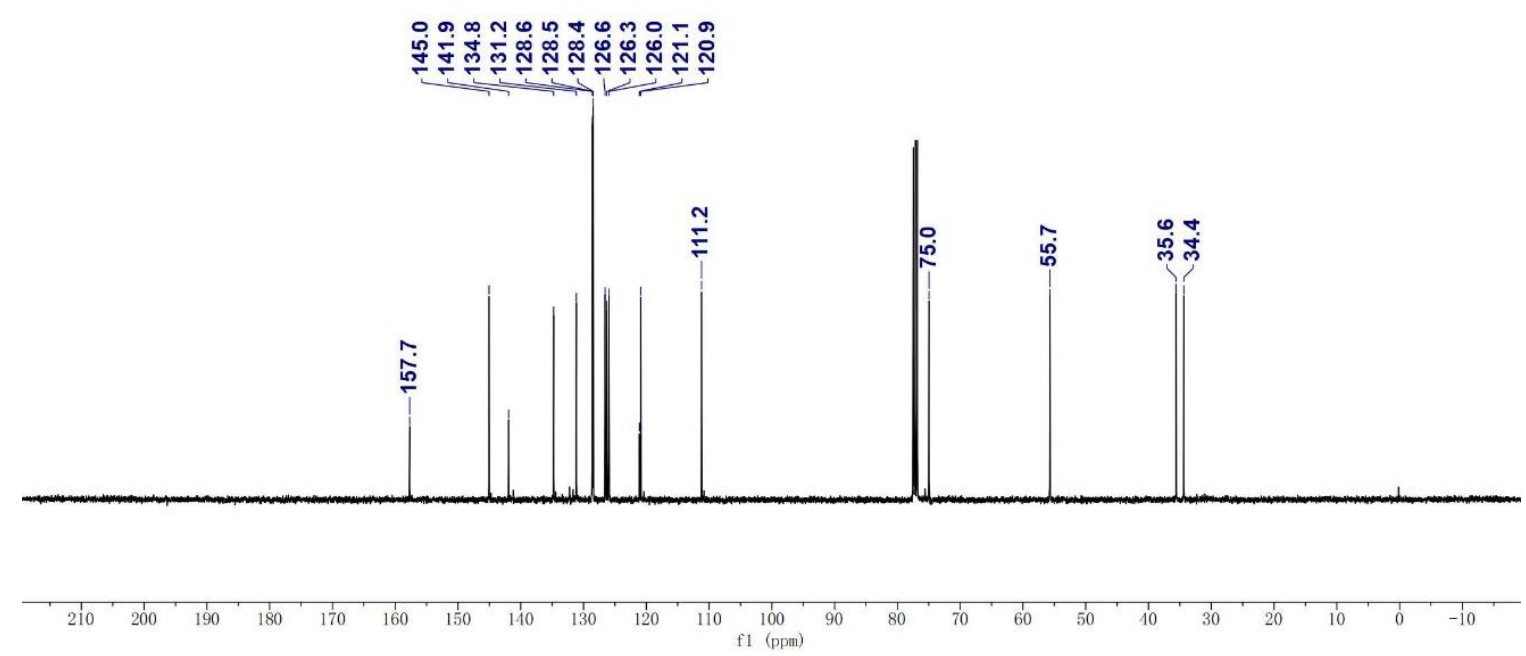

${ }^{13} \mathrm{C}$ NMR spectrum of $\mathbf{1 h}$ was measured in $\mathrm{CDCl}_{3}$ at $101 \mathrm{MHz}$ 


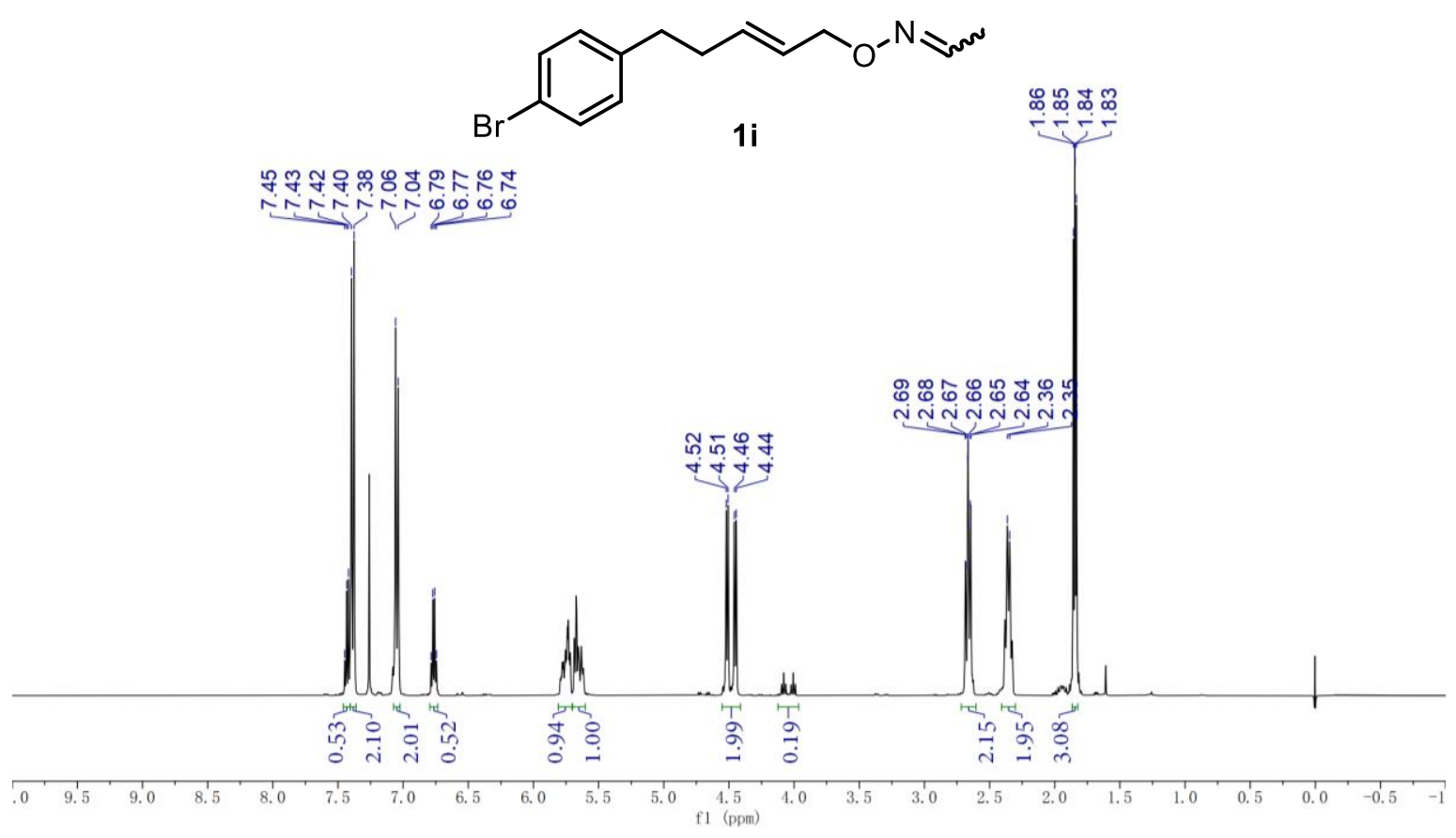

${ }^{1} \mathrm{H}$ NMR spectrum of $\mathbf{1 i}$ was measured in $\mathrm{CDCl}_{3}$ at $400 \mathrm{MH}$
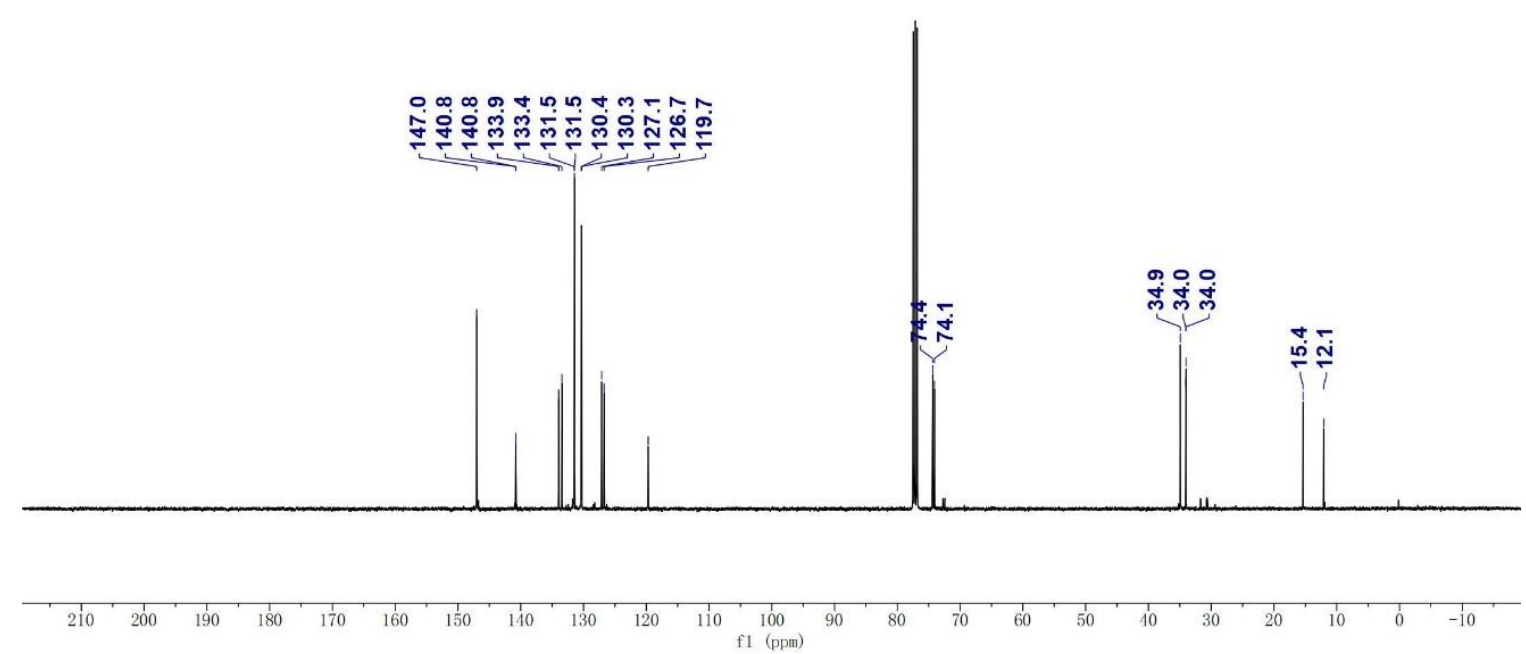

${ }^{13} \mathrm{C}$ NMR spectrum of $1 \mathbf{i}$ was measured in $\mathrm{CDCl}_{3}$ at $101 \mathrm{MHz}$ 


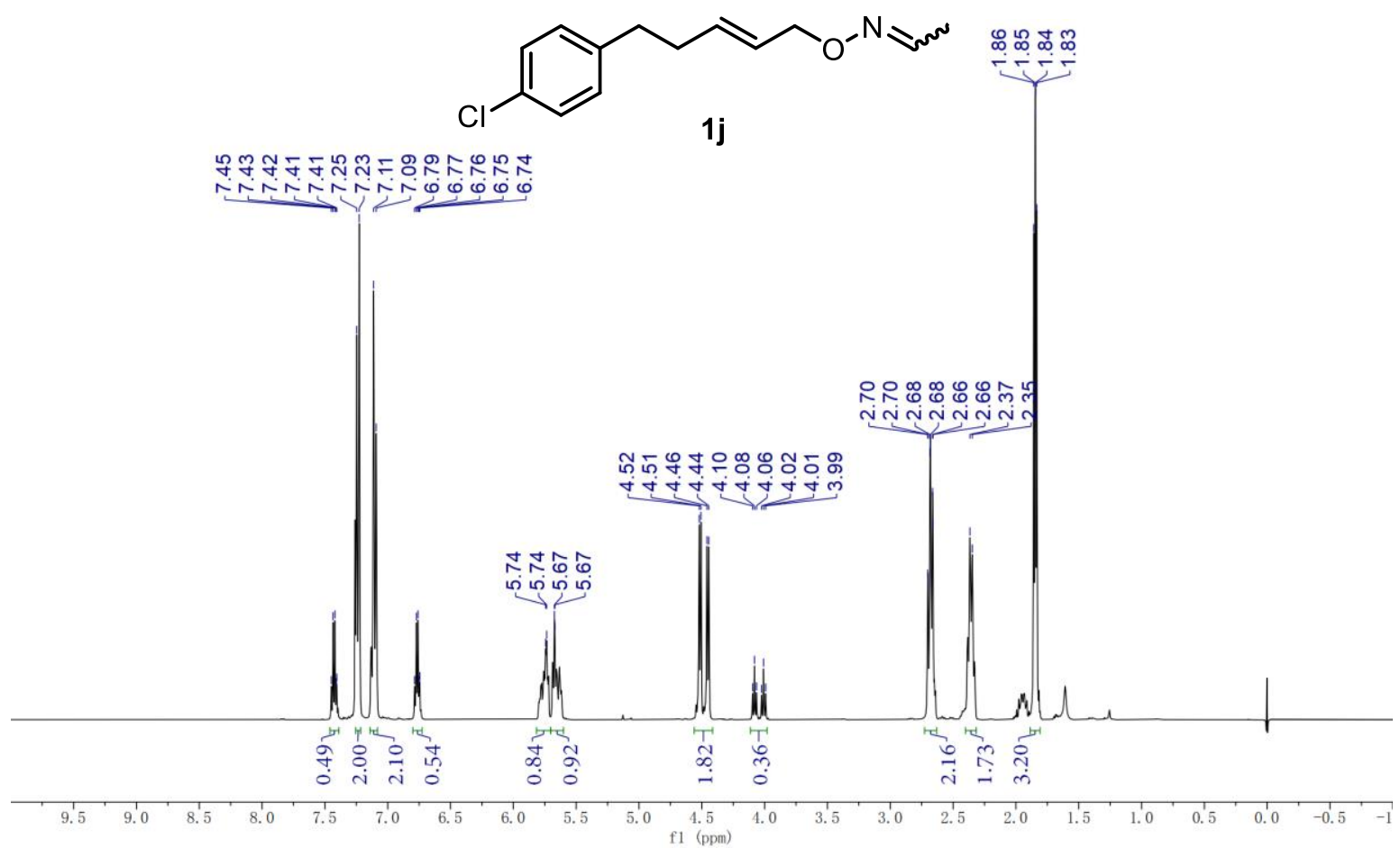

${ }^{1} \mathrm{H}$ NMR spectrum of $\mathbf{1} \mathbf{j}$ was measured in $\mathrm{CDCl}_{3}$ at $400 \mathrm{MH}$

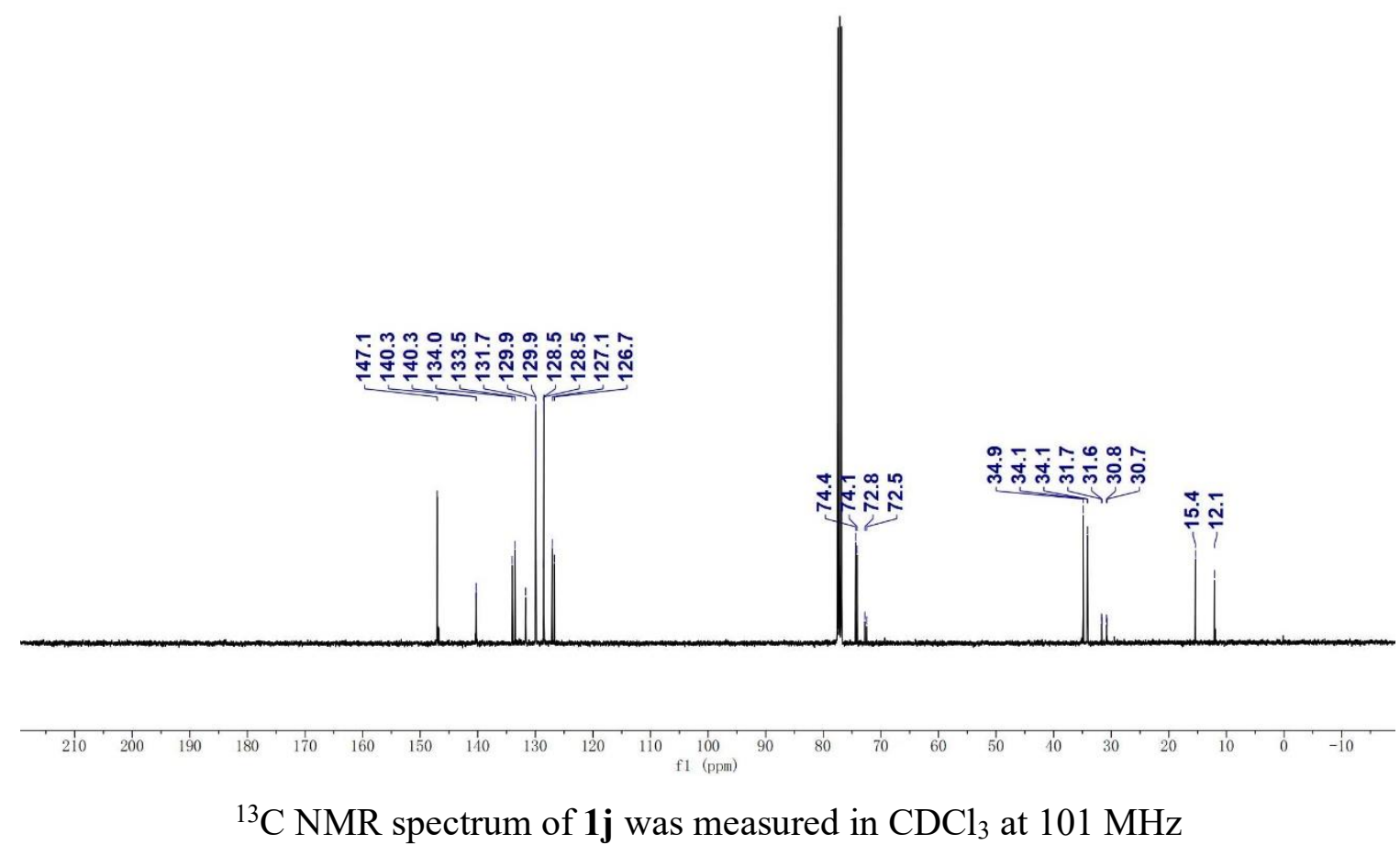


<smiles>CC=NOCC=CCCc1ccc(C(F)(F)F)cc1</smiles>

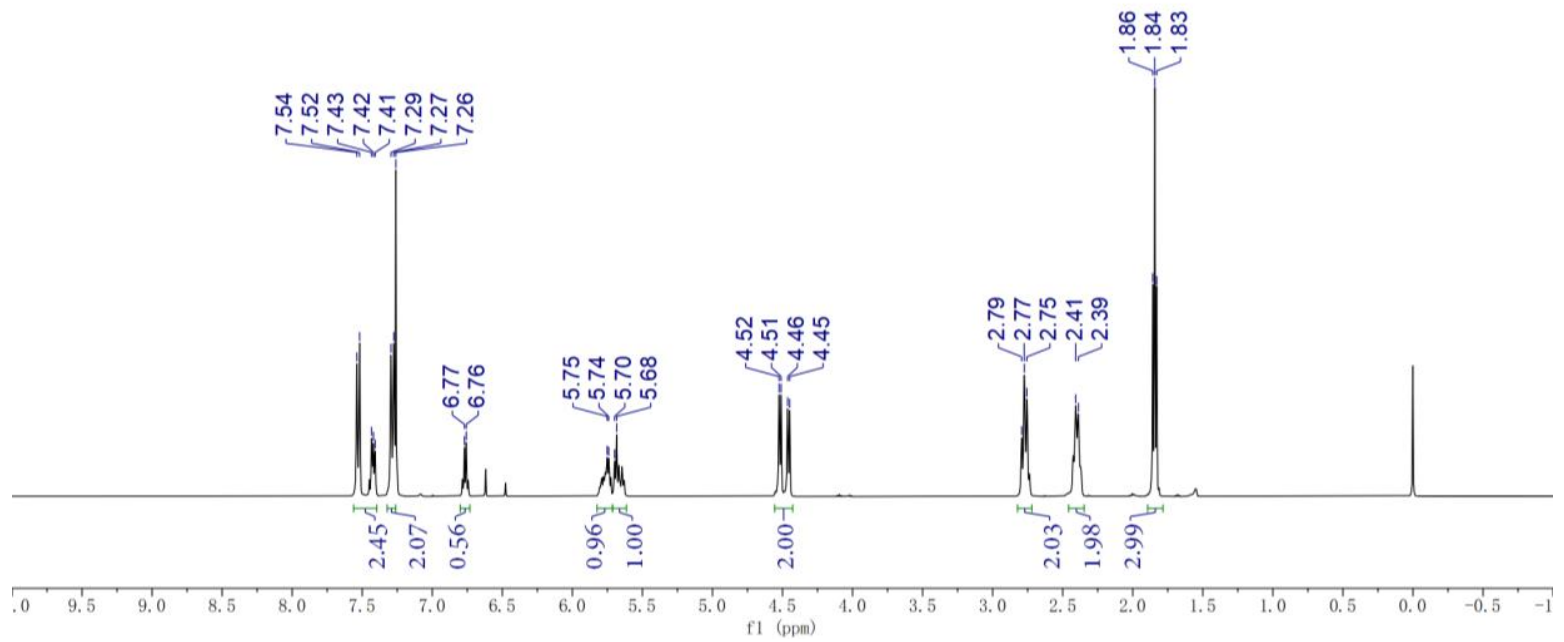

${ }^{1} \mathrm{H}$ NMR spectrum of $\mathbf{1 k}$ was measured in $\mathrm{CDCl}_{3}$ at $400 \mathrm{MH}$
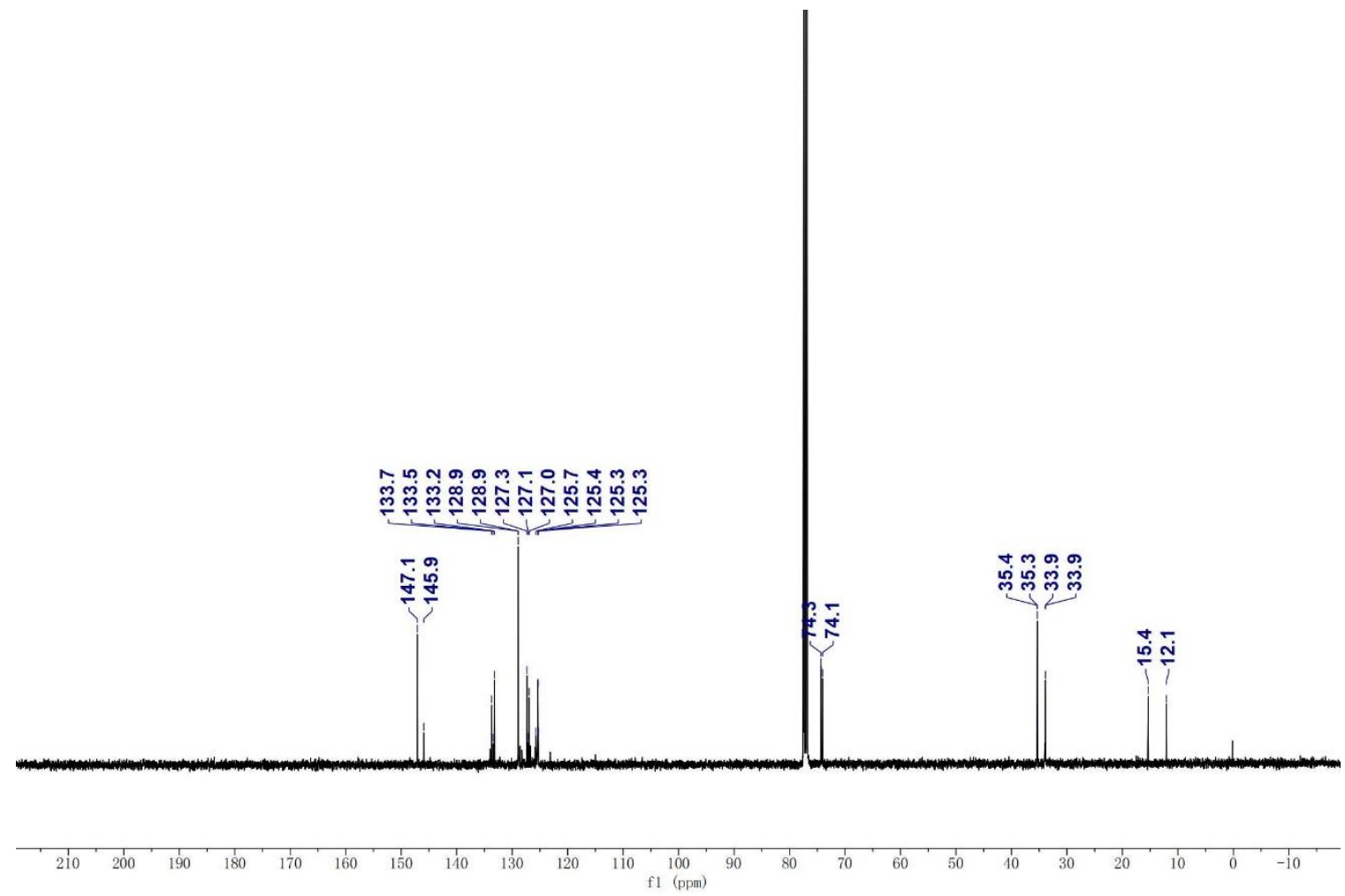

${ }^{13} \mathrm{C}$ NMR spectrum of $\mathbf{1 k}$ was measured in $\mathrm{CDCl}_{3}$ at $101 \mathrm{MHz}$ 


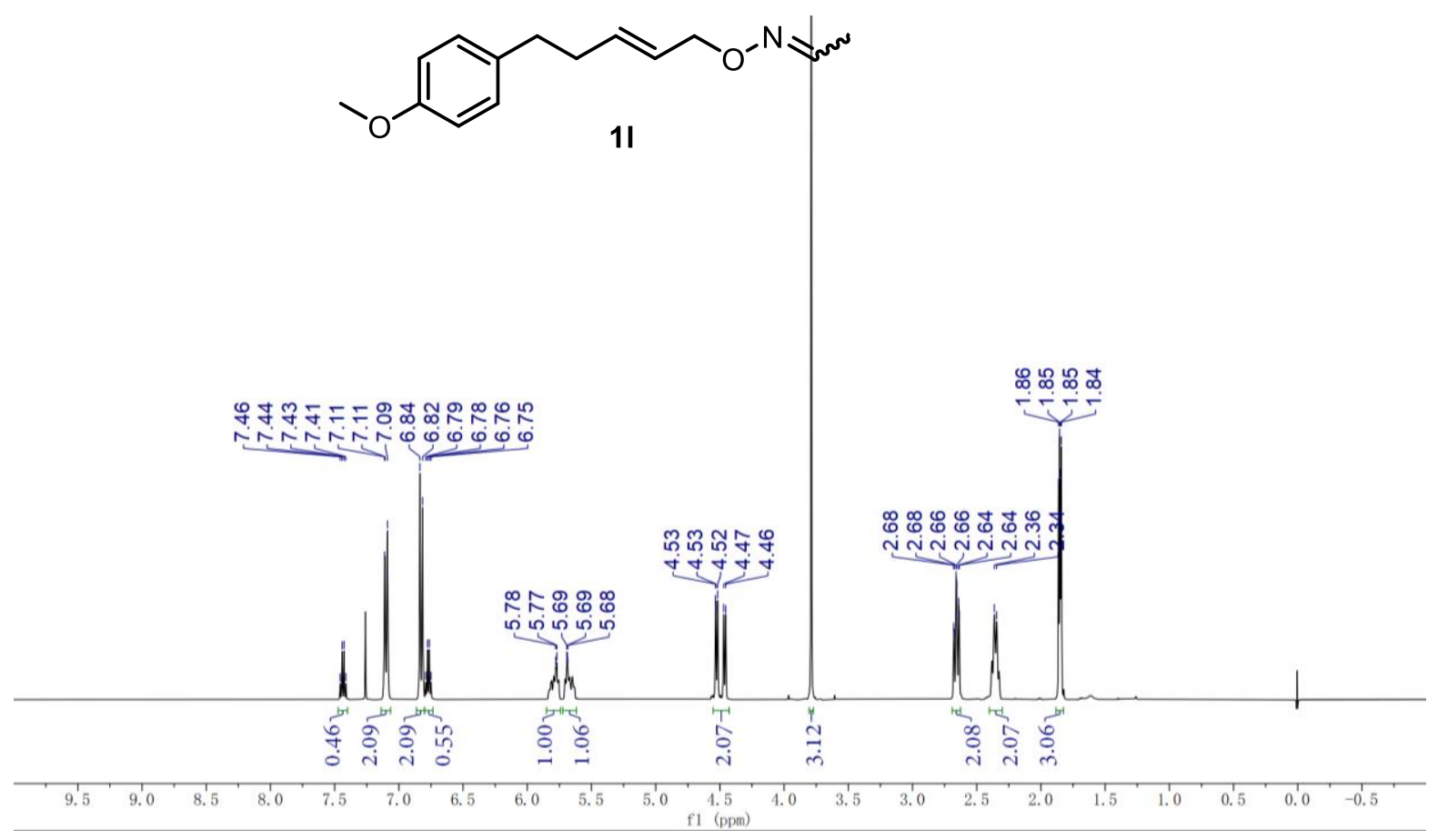

${ }^{1} \mathrm{H}$ NMR spectrum of 11 was measured in $\mathrm{CDCl}_{3}$ at $400 \mathrm{MH}$
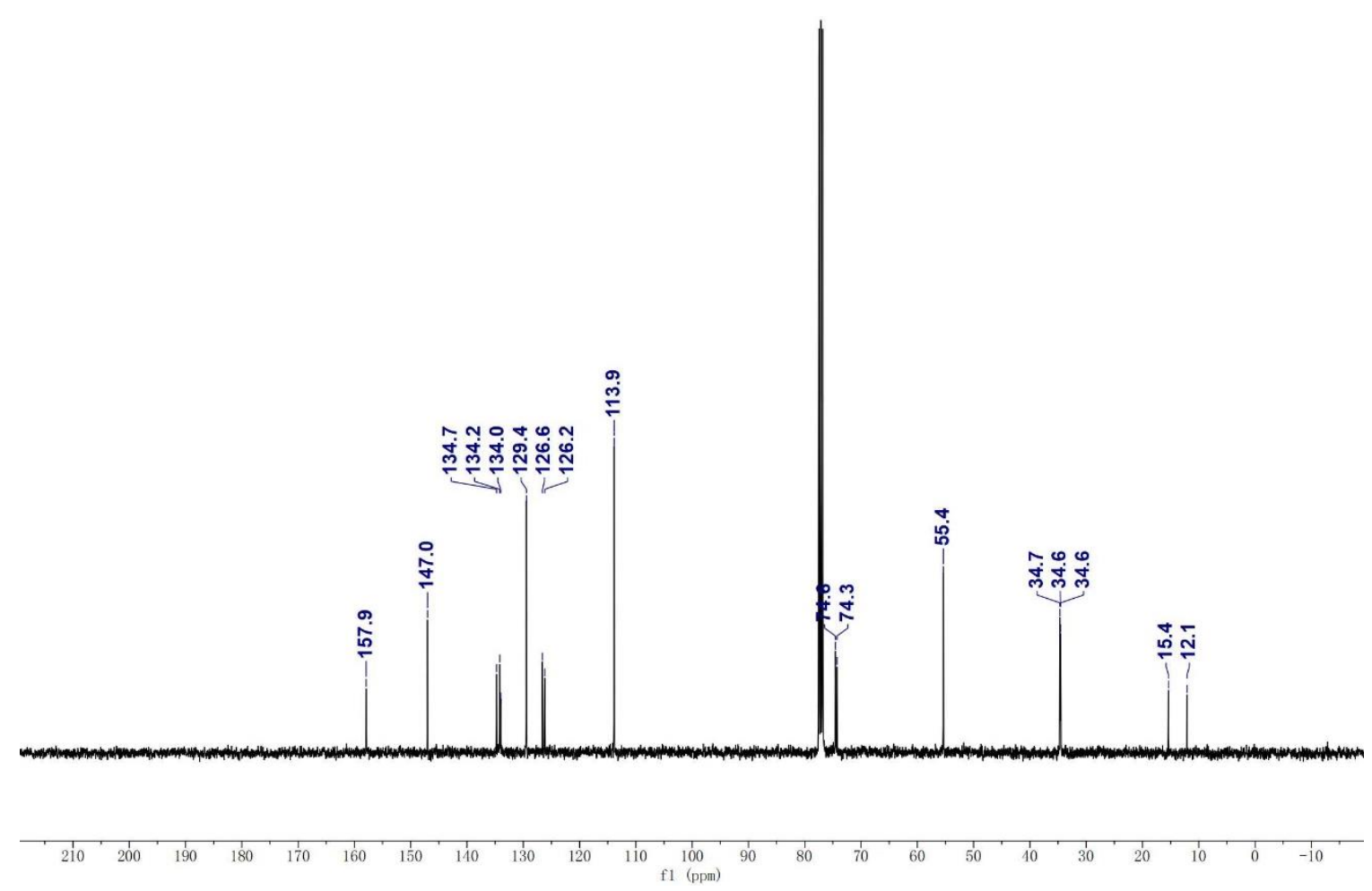

${ }^{13} \mathrm{C}$ NMR spectrum of 11 was measured in $\mathrm{CDCl}_{3}$ at $101 \mathrm{MHz}$ 


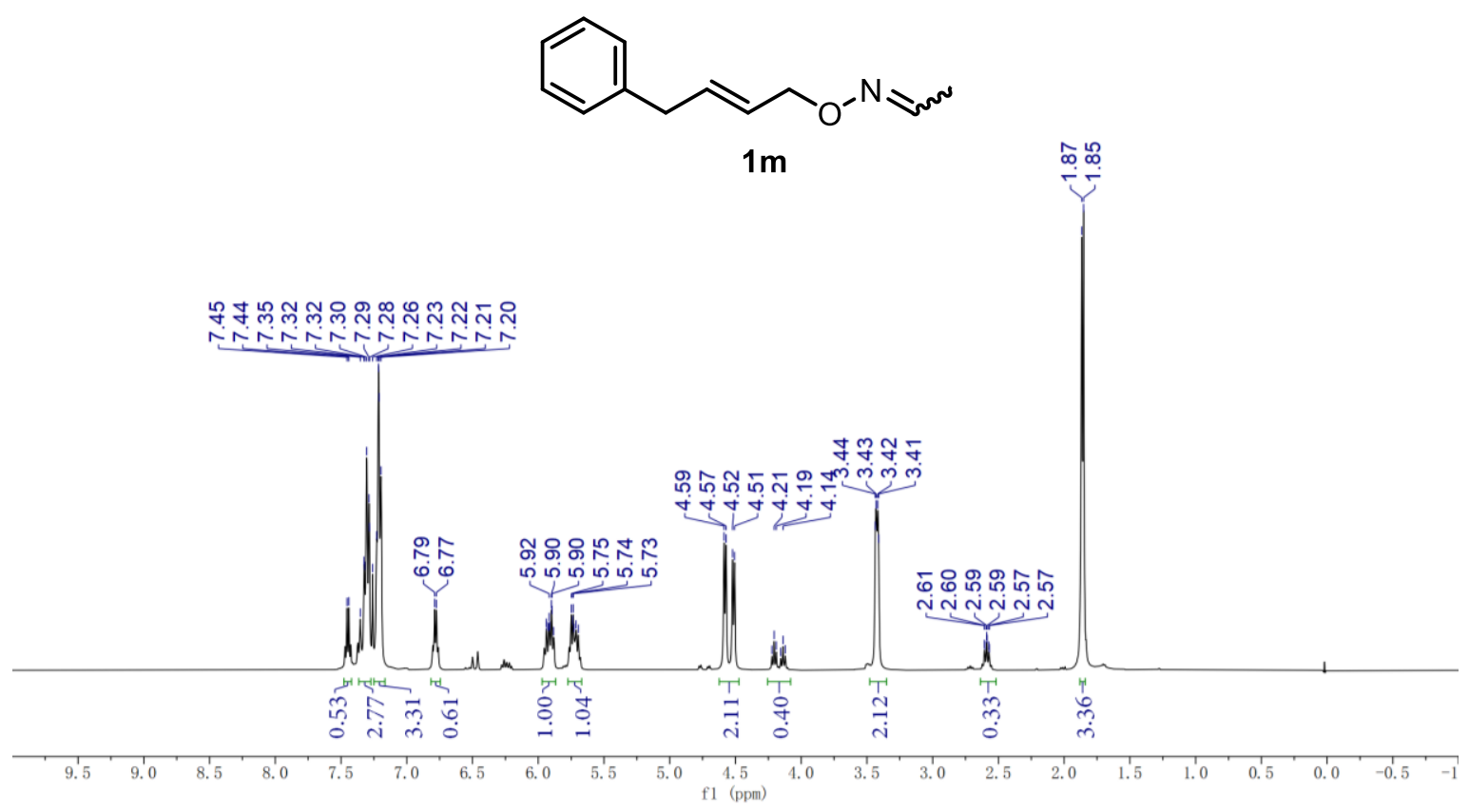

${ }^{1} \mathrm{H}$ NMR spectrum of $\mathbf{1 m}$ was measured in $\mathrm{CDCl}_{3}$ at $400 \mathrm{MH}$
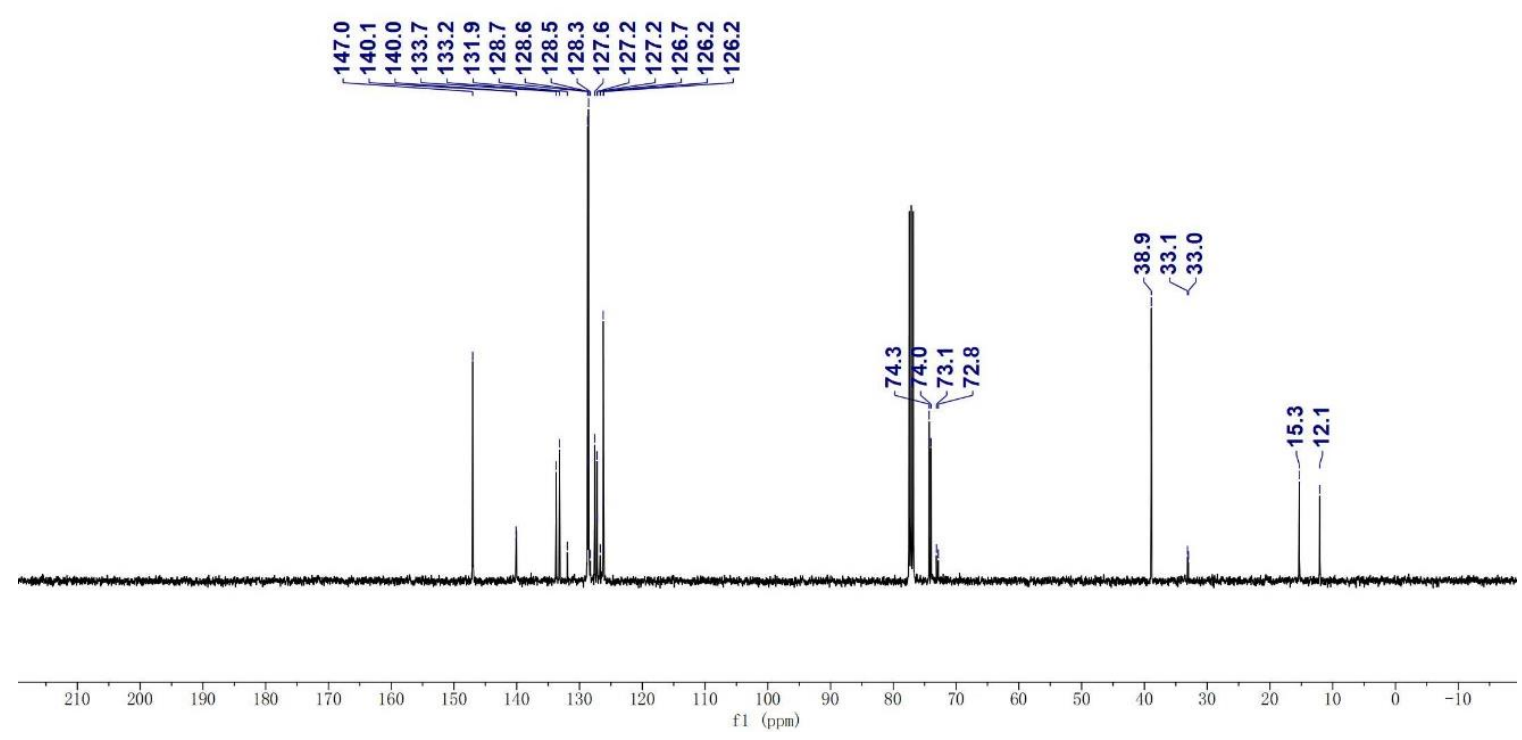

${ }^{13} \mathrm{C}$ NMR spectrum of $\mathbf{1 m}$ was measured in $\mathrm{CDCl}_{3}$ at $101 \mathrm{MHz}$ 


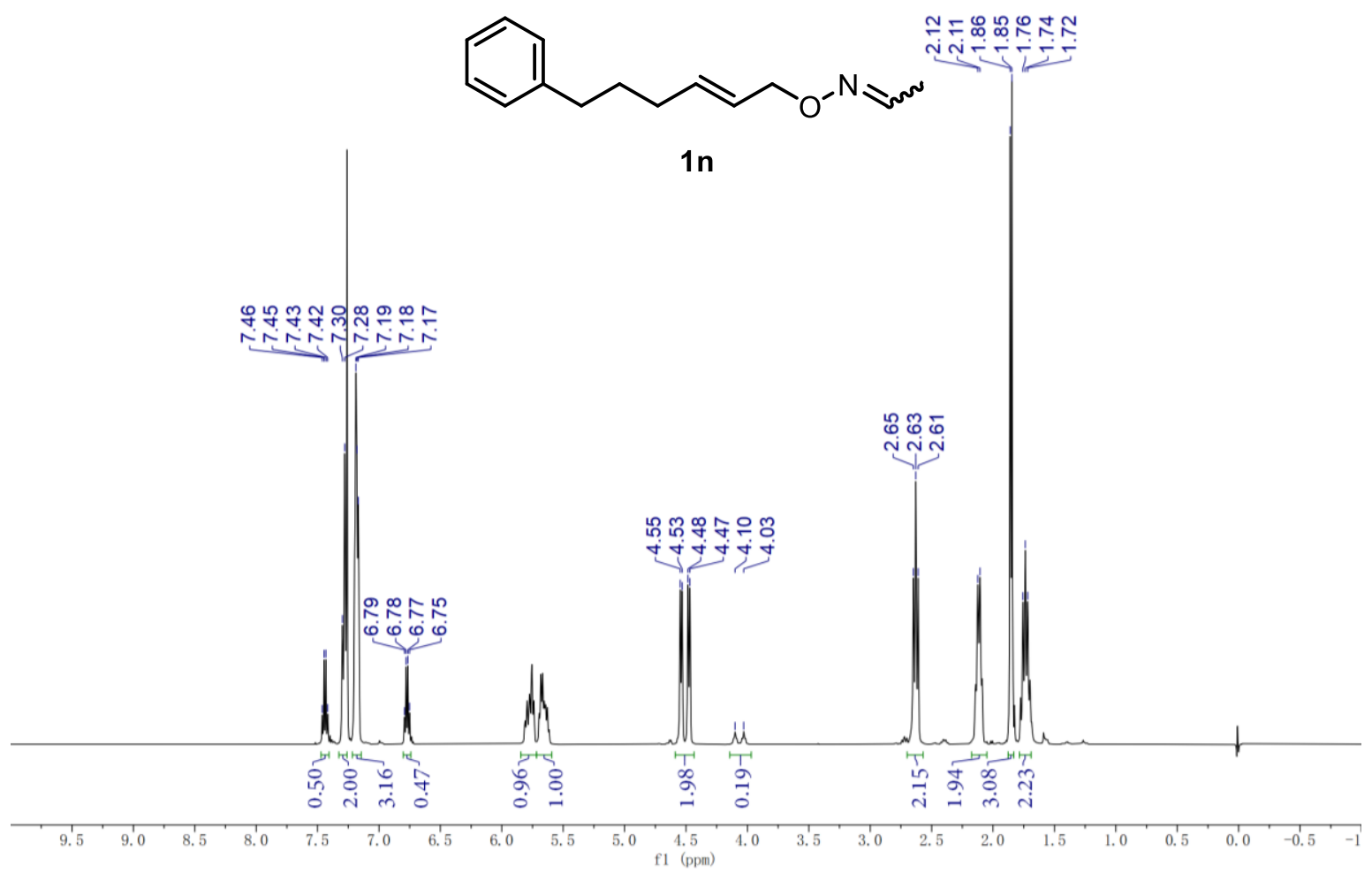

${ }^{1} \mathrm{H}$ NMR spectrum of $\mathbf{1 n}$ was measured in $\mathrm{CDCl}_{3}$ at $400 \mathrm{MH}$
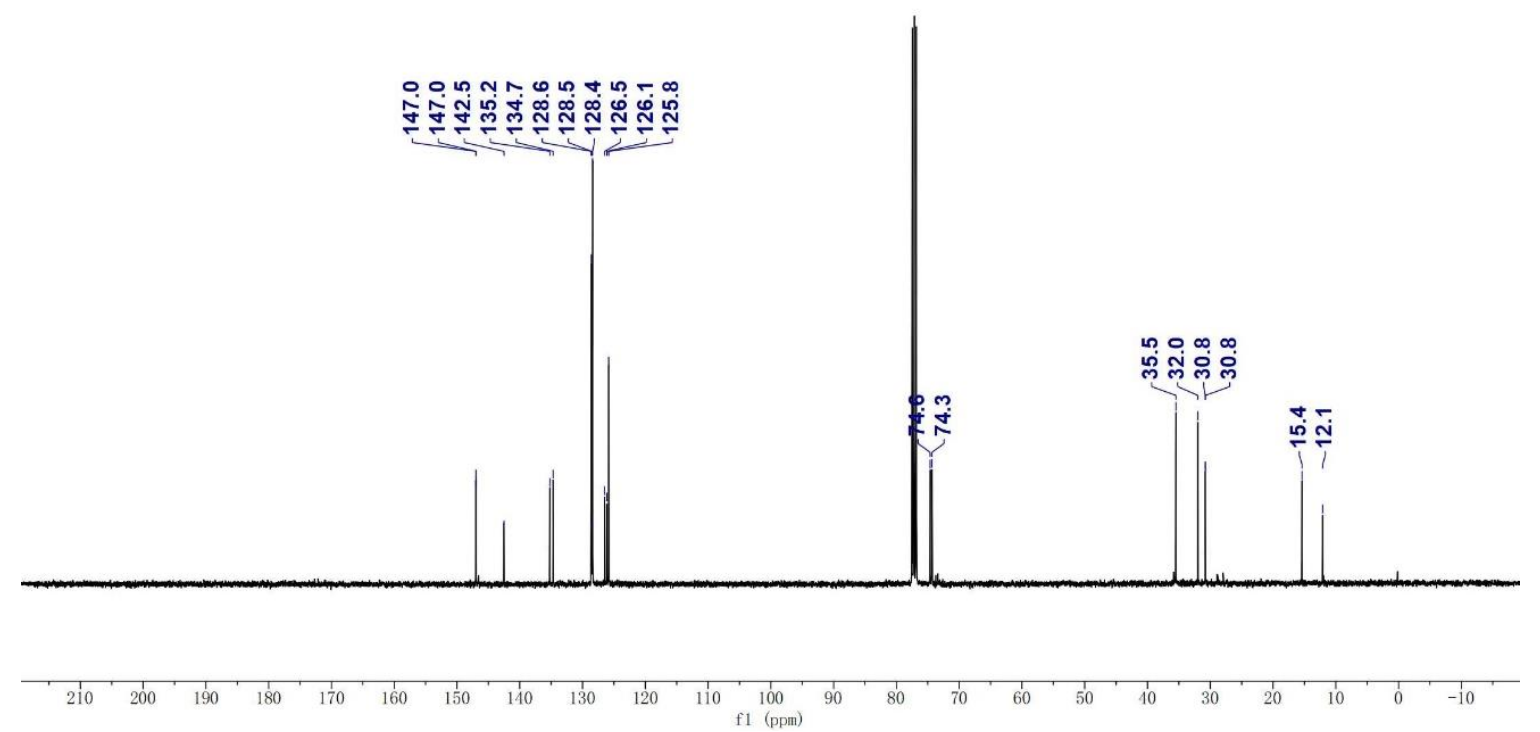

${ }^{13} \mathrm{C}$ NMR spectrum of $\mathbf{1 n}$ was measured in $\mathrm{CDCl}_{3}$ at $101 \mathrm{MHz}$ 


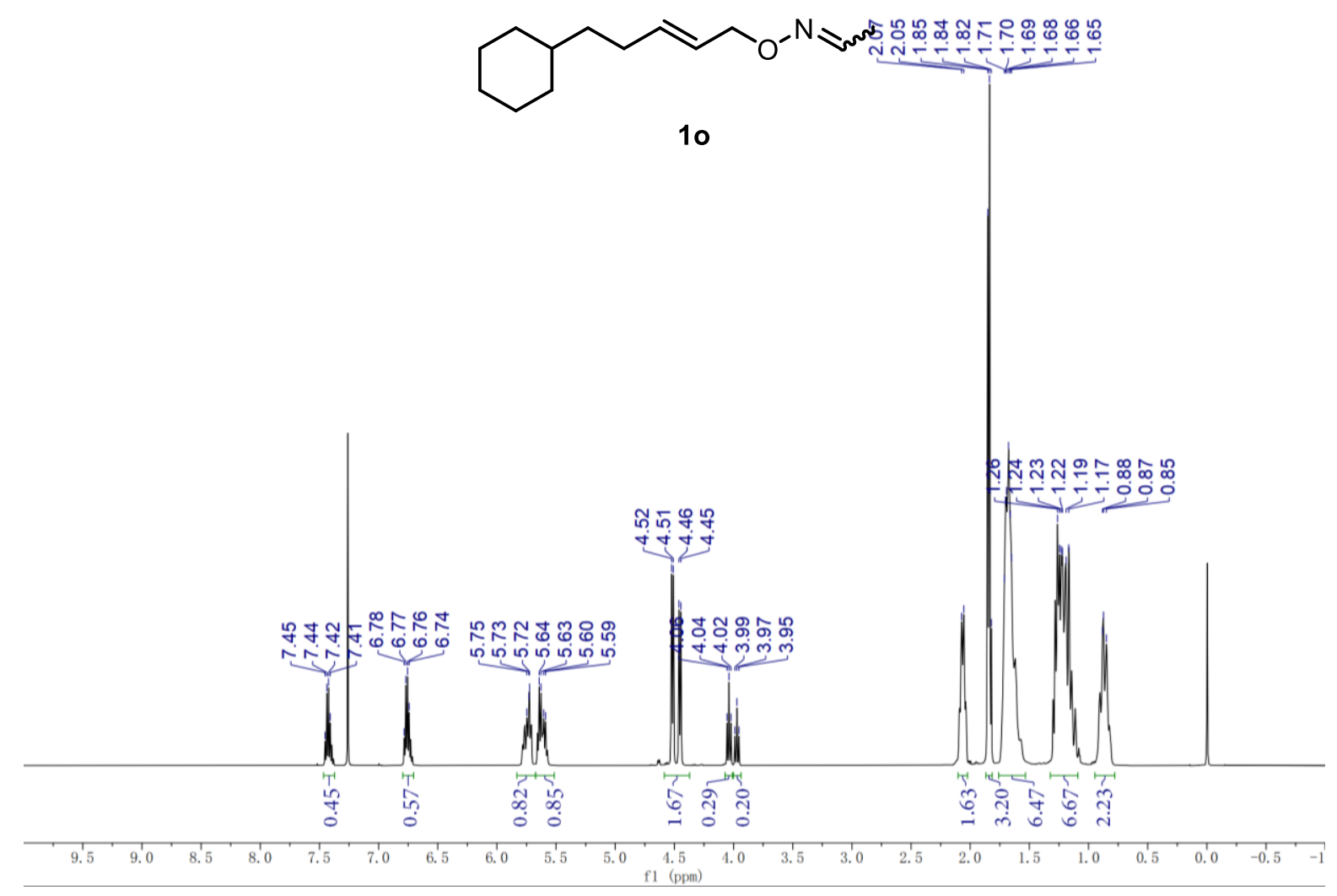

${ }^{1} \mathrm{H}$ NMR spectrum of 10 was measured in $\mathrm{CDCl}_{3}$ at $400 \mathrm{MH}$
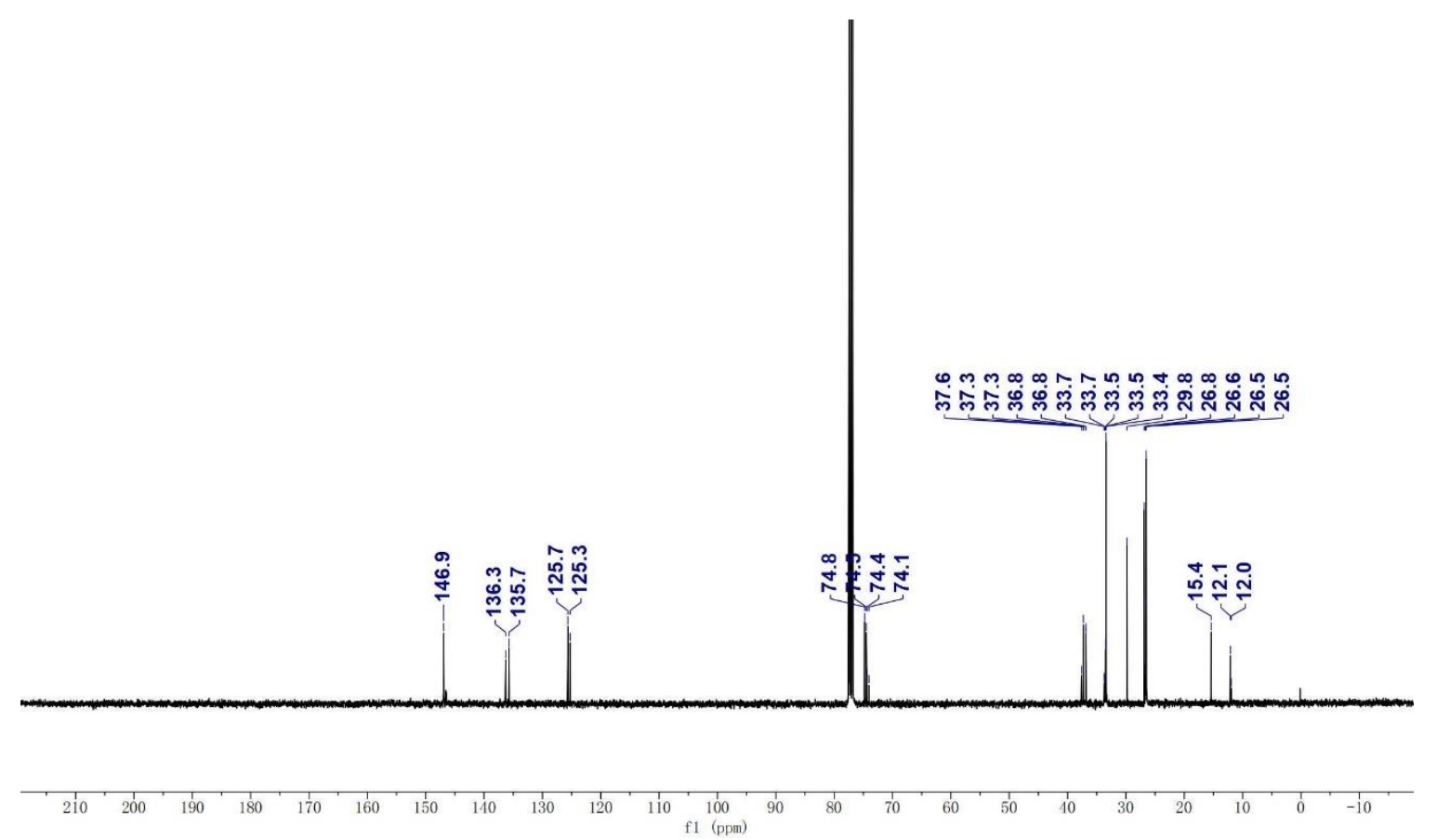

${ }^{13} \mathrm{C}$ NMR spectrum of 10 was measured in $\mathrm{CDCl}_{3}$ at $101 \mathrm{MHz}$ 


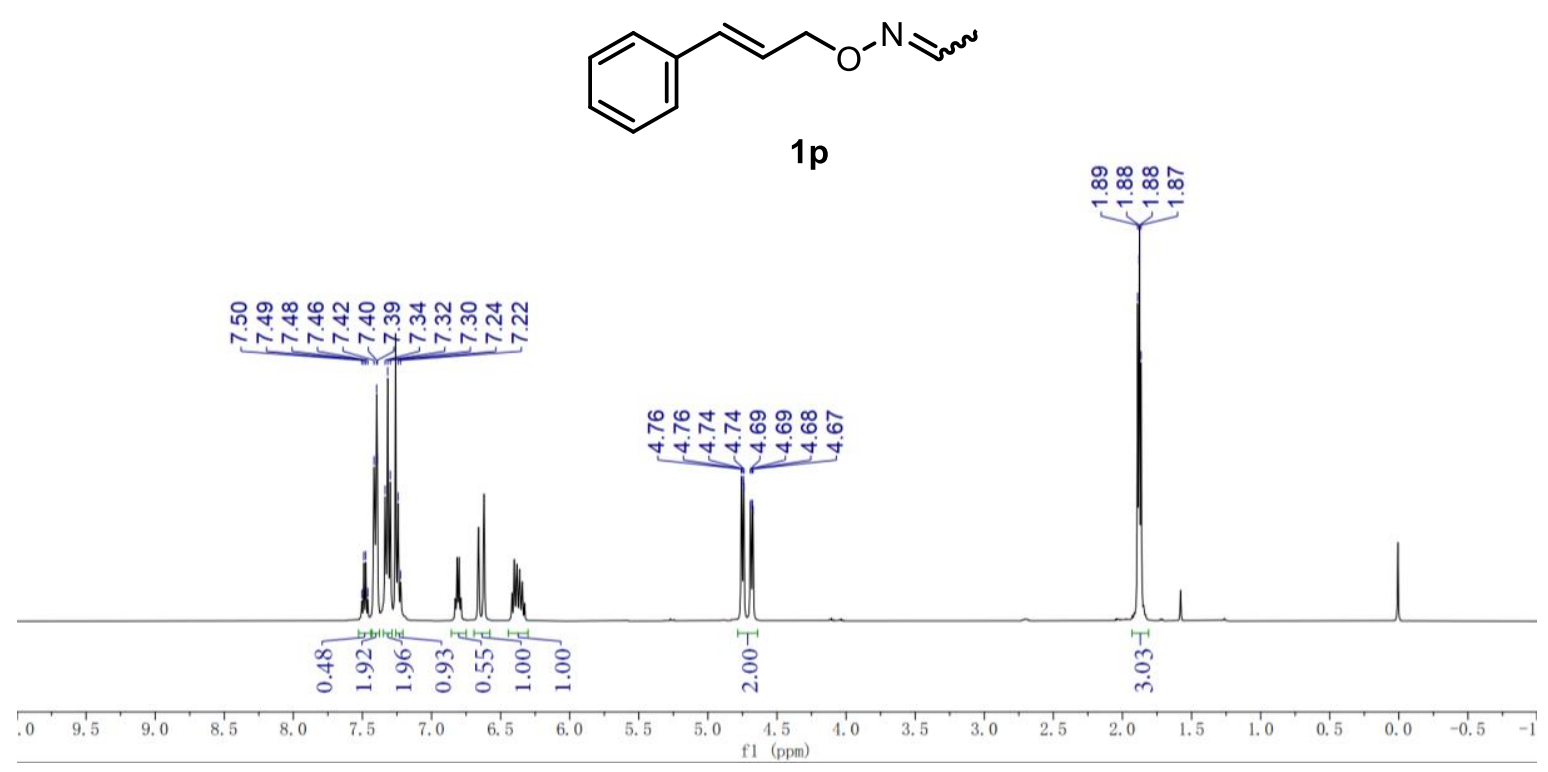

${ }^{1} \mathrm{H}$ NMR spectrum of $1 p$ was measured in $\mathrm{CDCl}_{3}$ at $400 \mathrm{MH}$
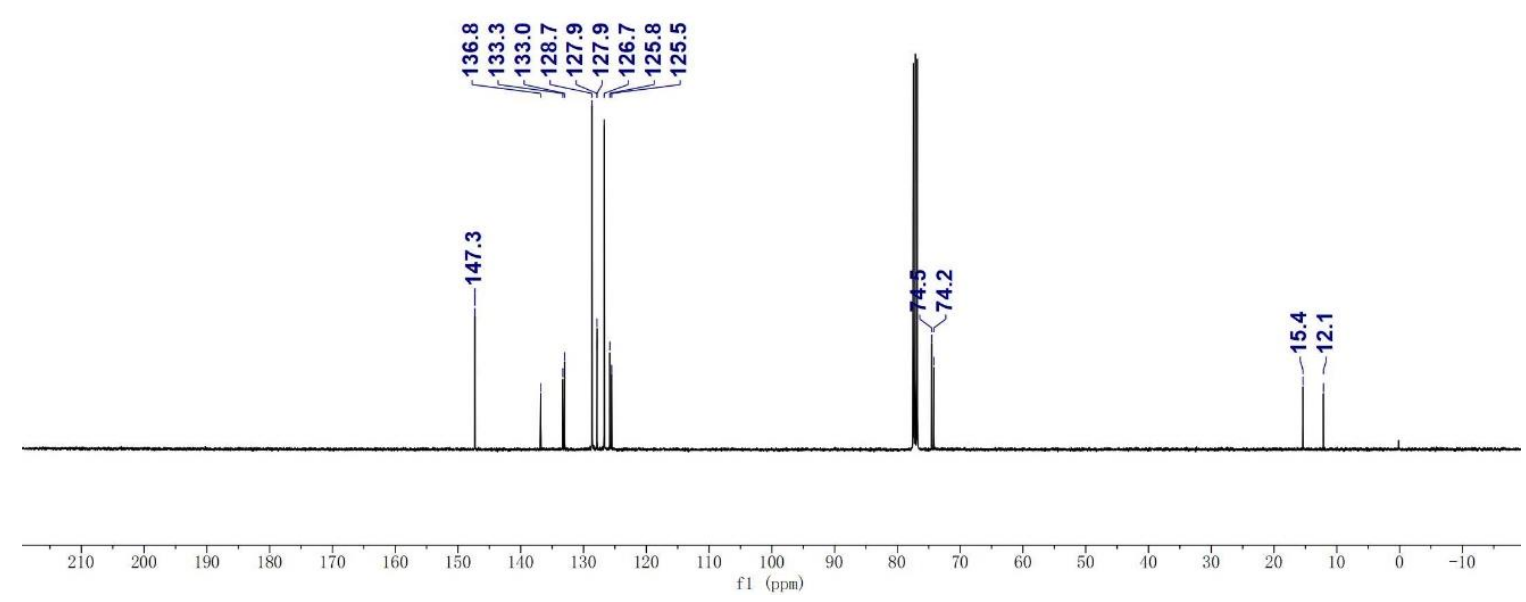

${ }^{13} \mathrm{C}$ NMR spectrum of $\mathbf{1 p}$ was measured in $\mathrm{CDCl}_{3}$ at $101 \mathrm{MHz}$ 


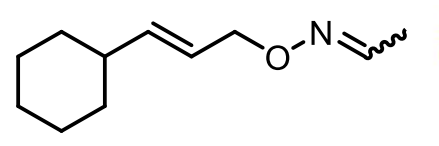

$1 q$

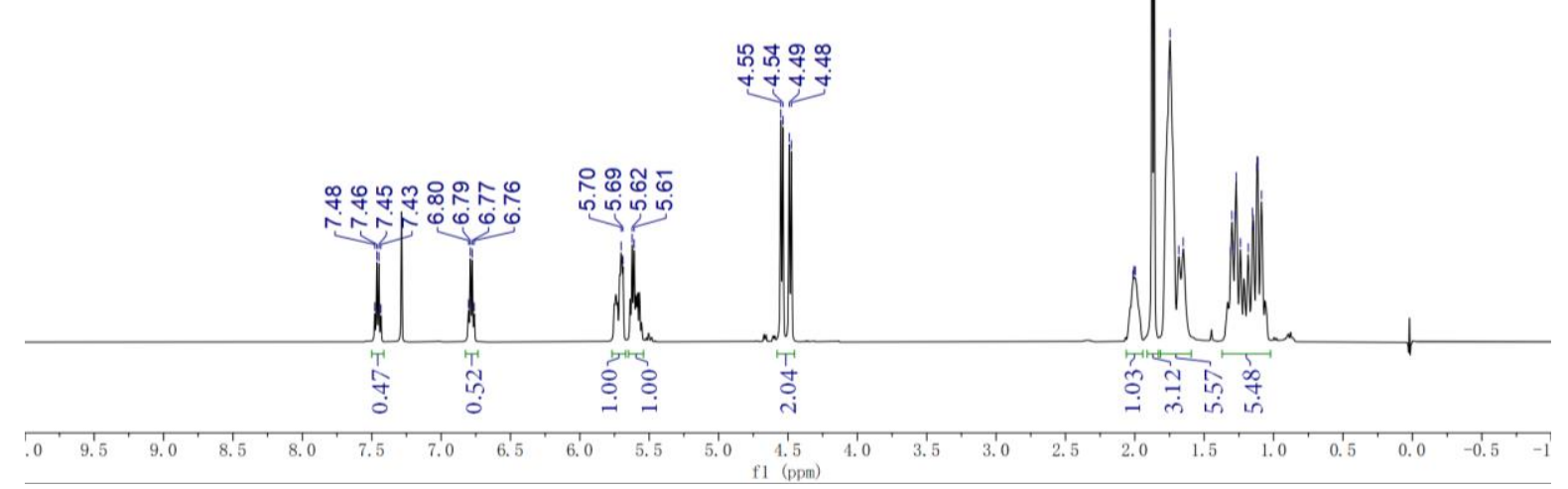

${ }^{1} \mathrm{H}$ NMR spectrum of $\mathbf{1 q}$ was measured in $\mathrm{CDCl}_{3}$ at $400 \mathrm{MH}$
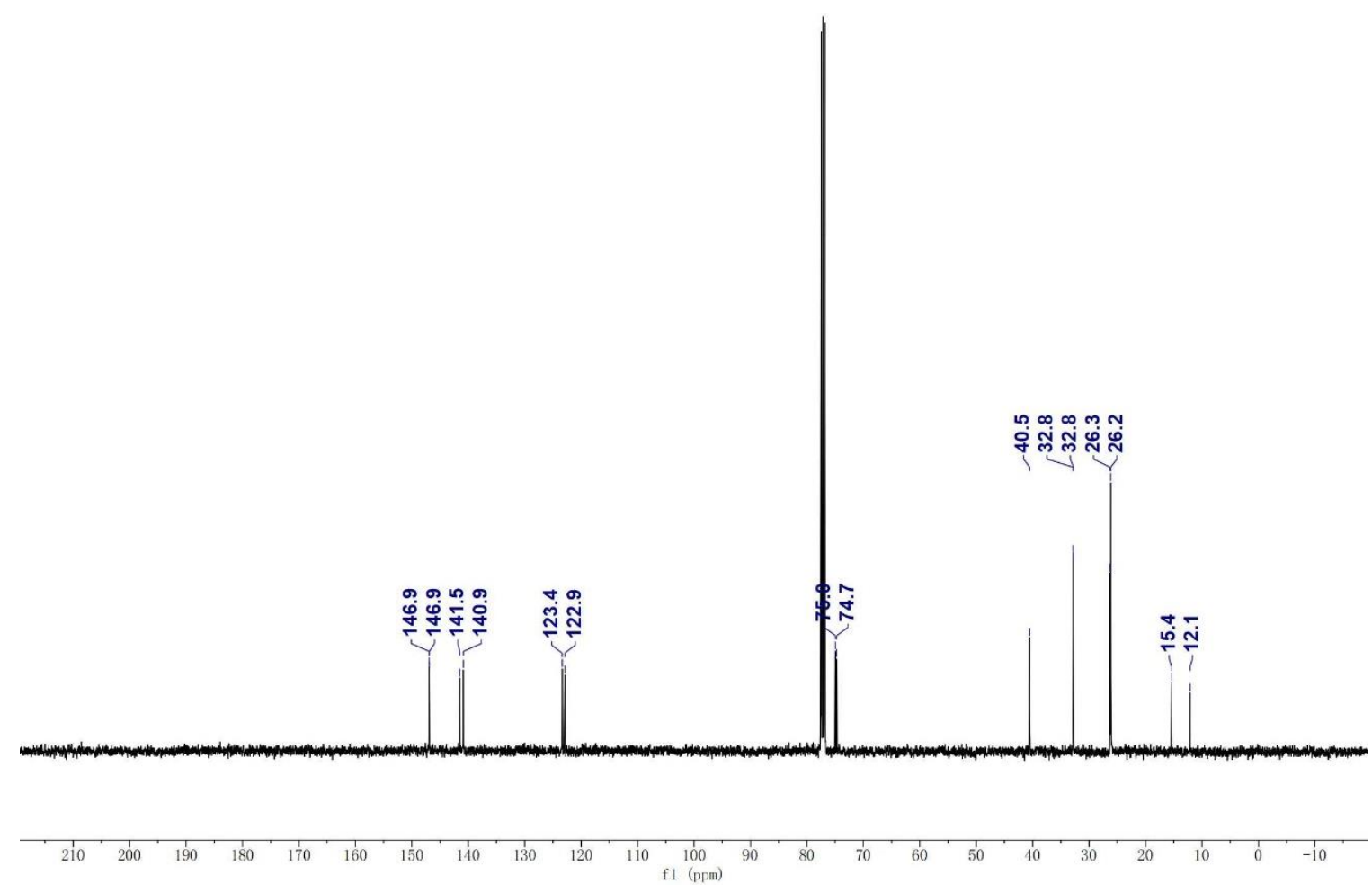

${ }^{13} \mathrm{C}$ NMR spectrum of $\mathbf{1 q}$ was measured in $\mathrm{CDCl}_{3}$ at $101 \mathrm{MHz}$ 


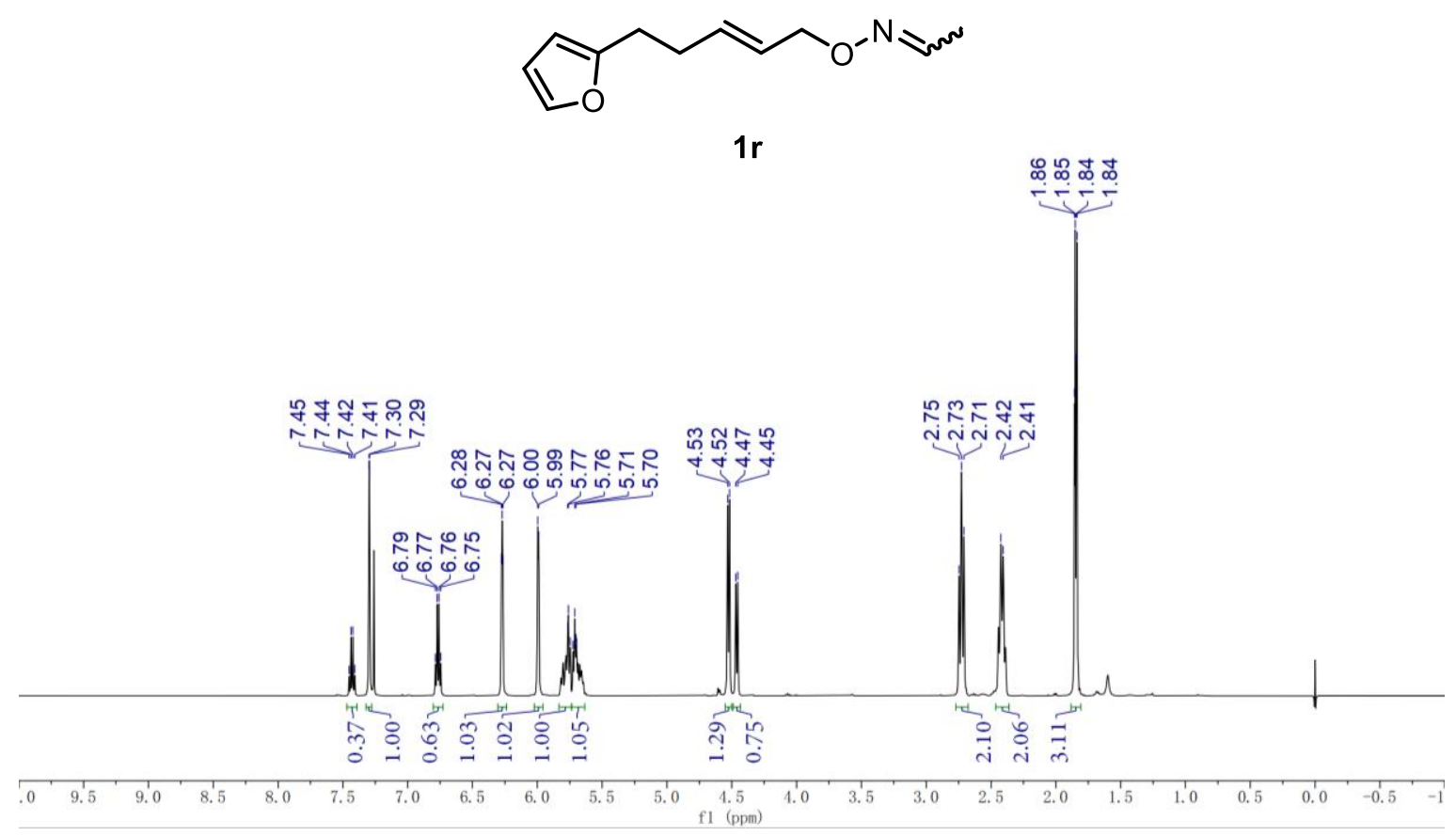

${ }^{1} \mathrm{H}$ NMR spectrum of $1 \mathbf{r}$ was measured in $\mathrm{CDCl}_{3}$ at $400 \mathrm{MH}$

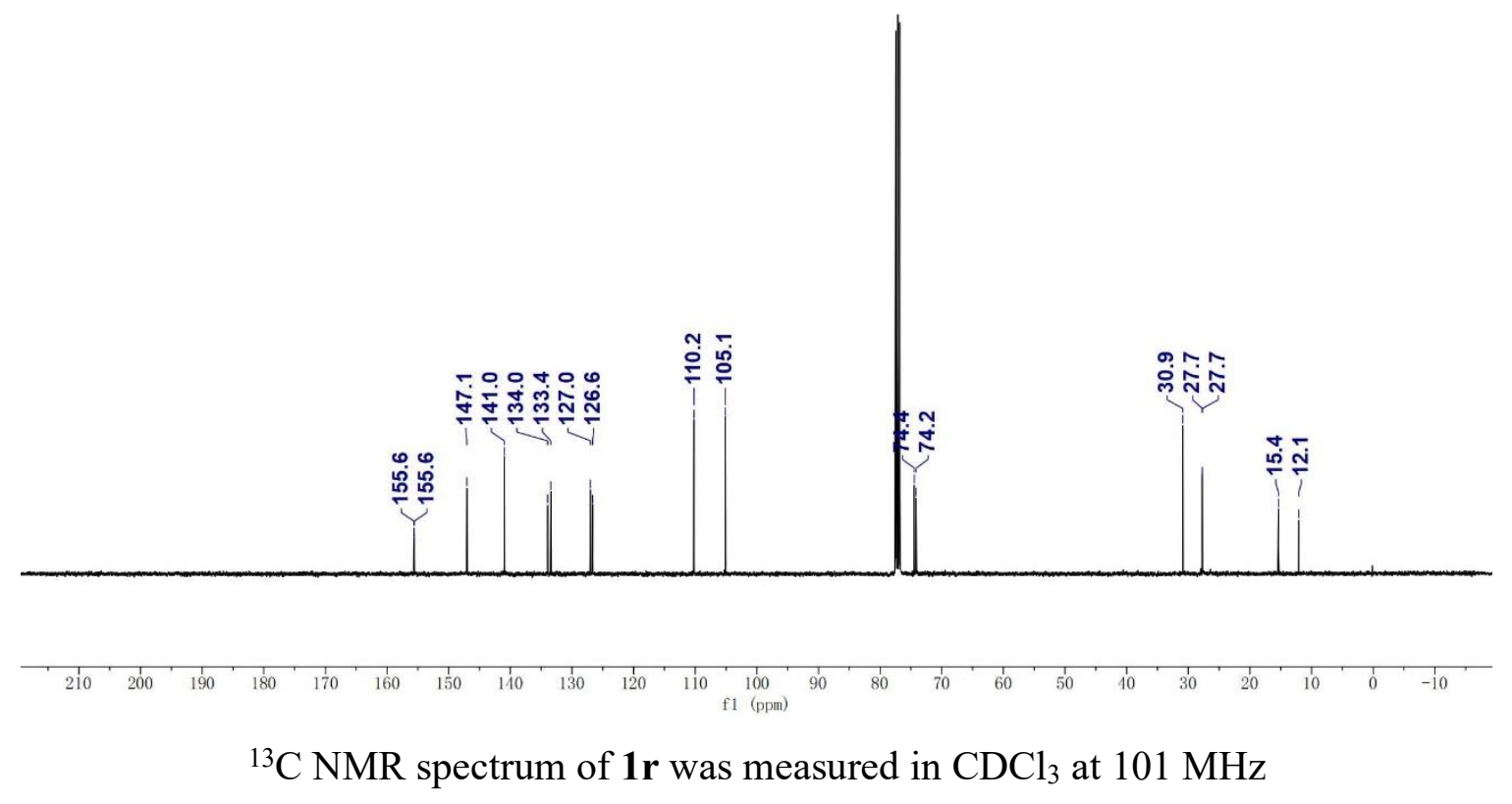




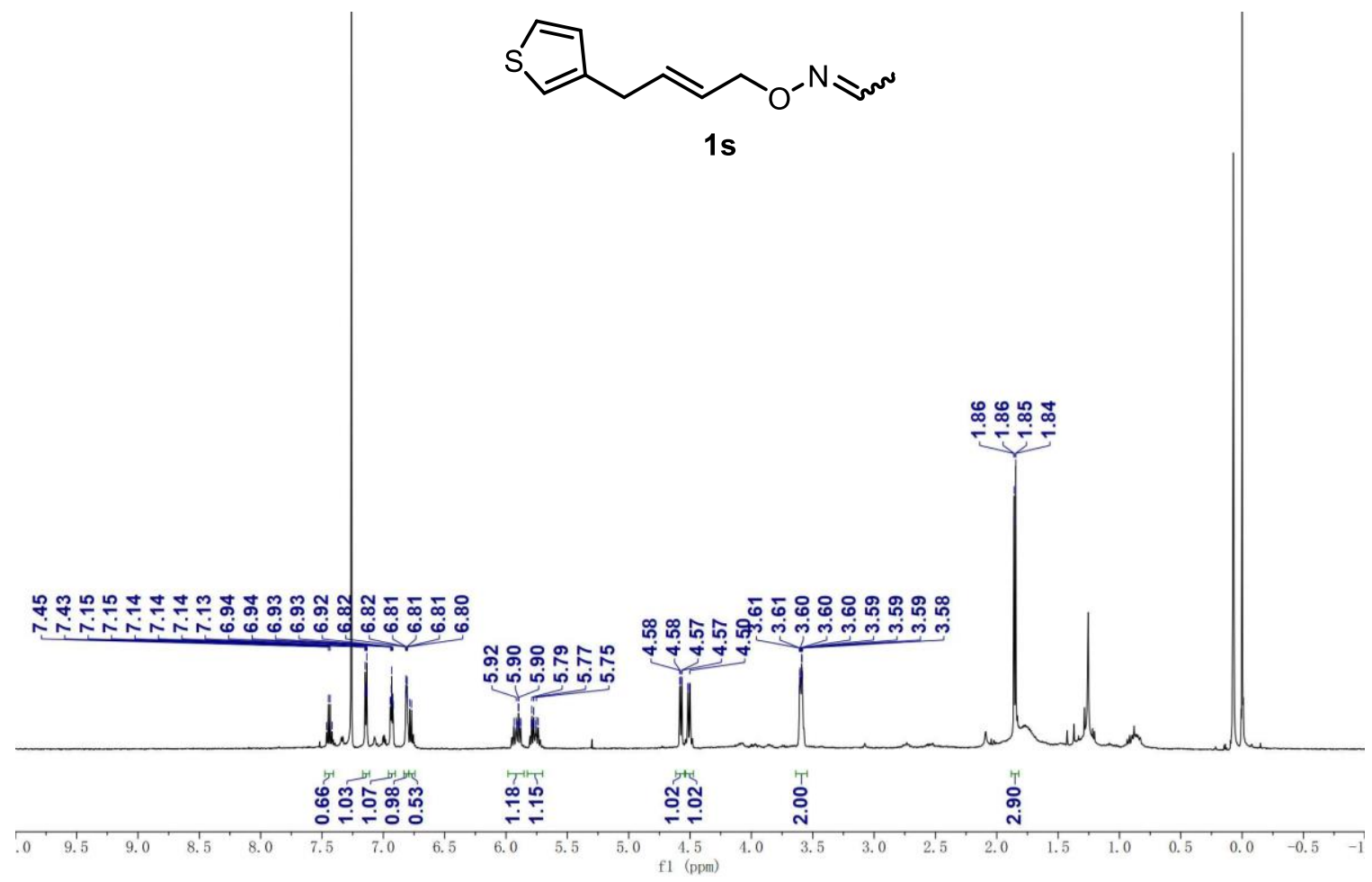

${ }^{1} \mathrm{H}$ NMR spectrum of $1 \mathrm{~s}$ was measured in $\mathrm{CDCl}_{3}$ at $400 \mathrm{MH}$
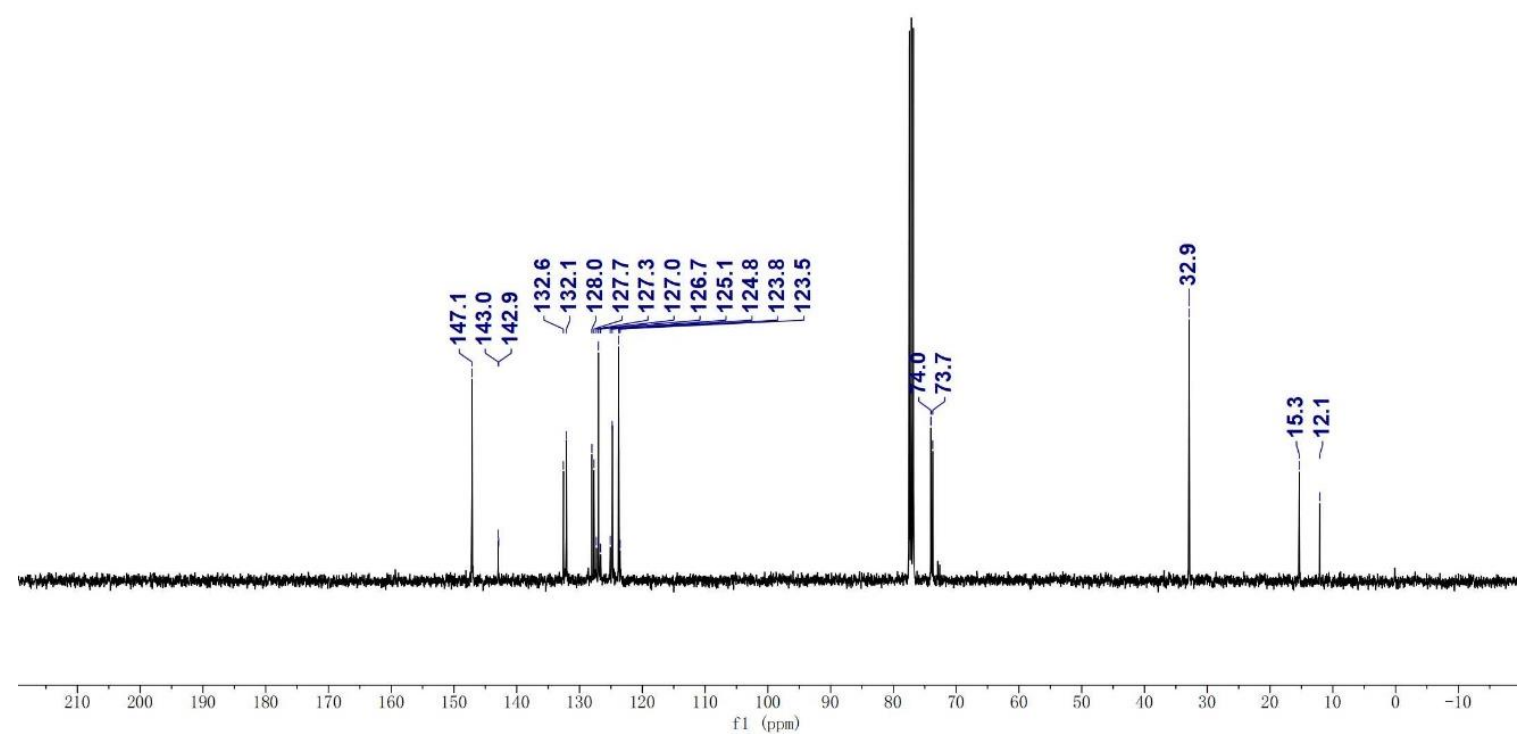

${ }^{13} \mathrm{C}$ NMR spectrum of 1 s was measured in $\mathrm{CDCl}_{3}$ at $101 \mathrm{MHz}$ 


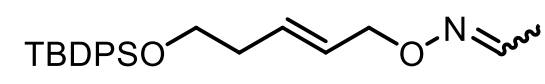

$1 t$

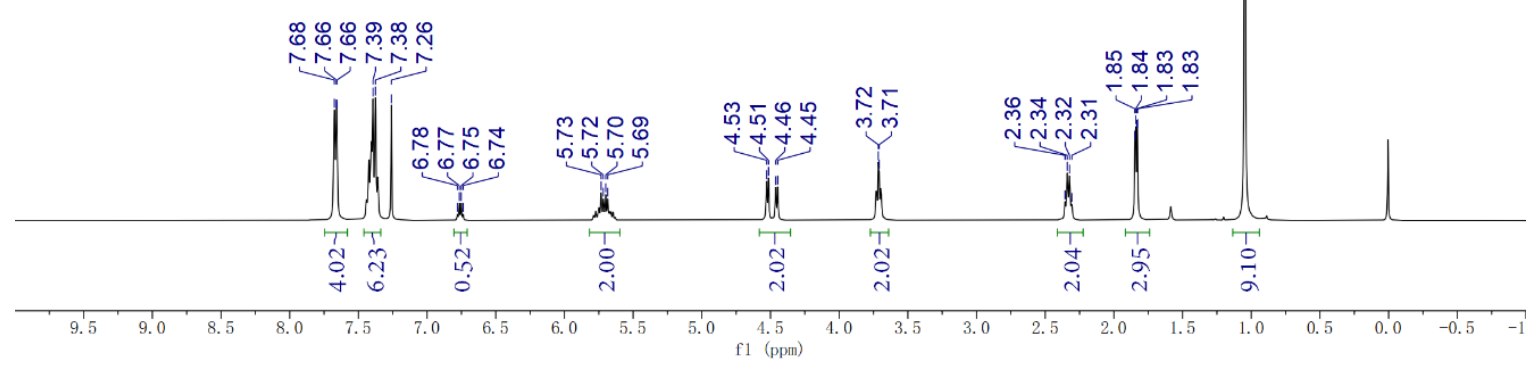

${ }^{1} \mathrm{H}$ NMR spectrum of $\mathbf{1 t}$ was measured in $\mathrm{CDCl}_{3}$ at $400 \mathrm{MH}$
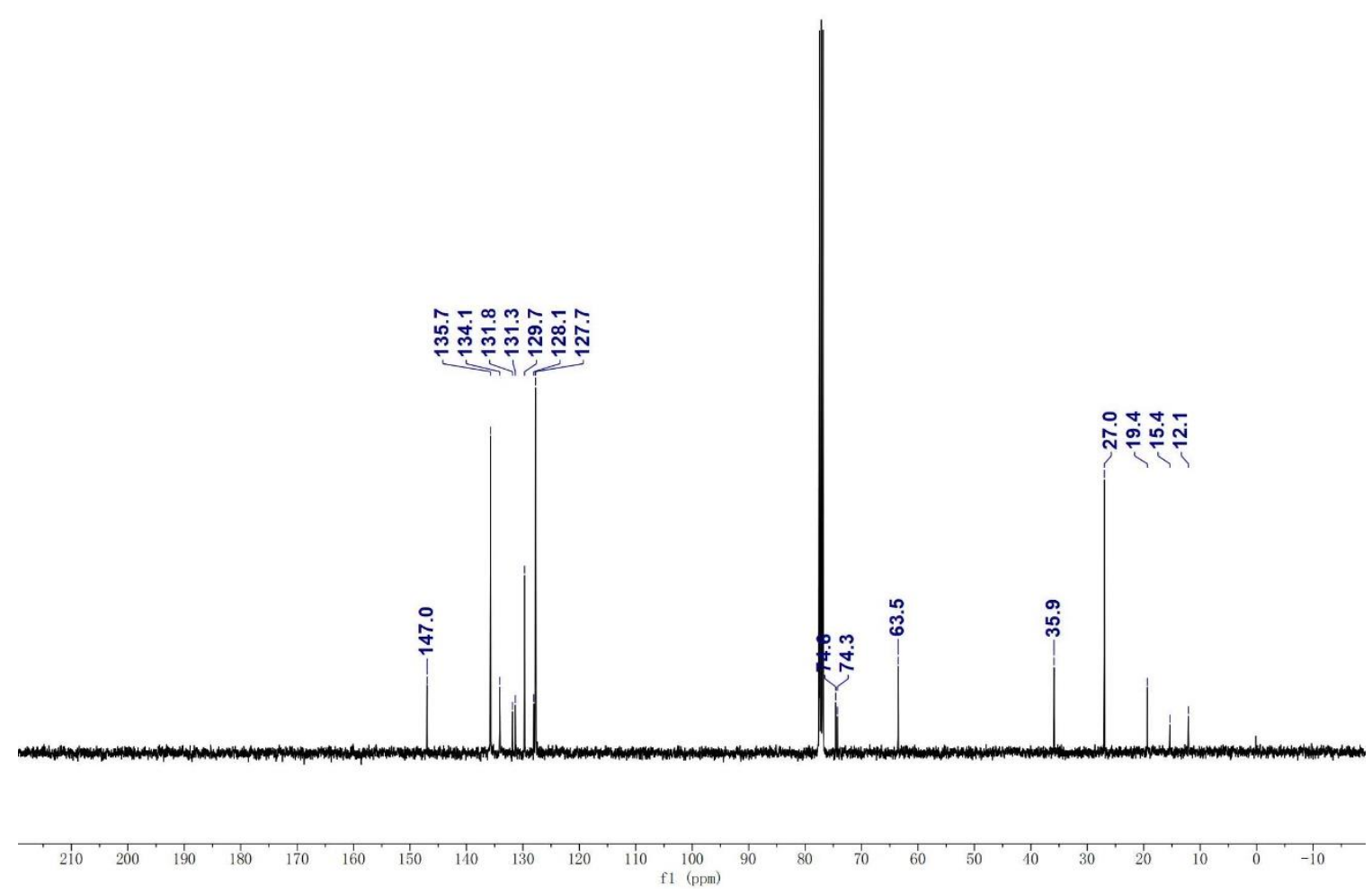

${ }^{13} \mathrm{C}$ NMR spectrum of $1 t$ was measured in $\mathrm{CDCl}_{3}$ at $101 \mathrm{MHz}$ 

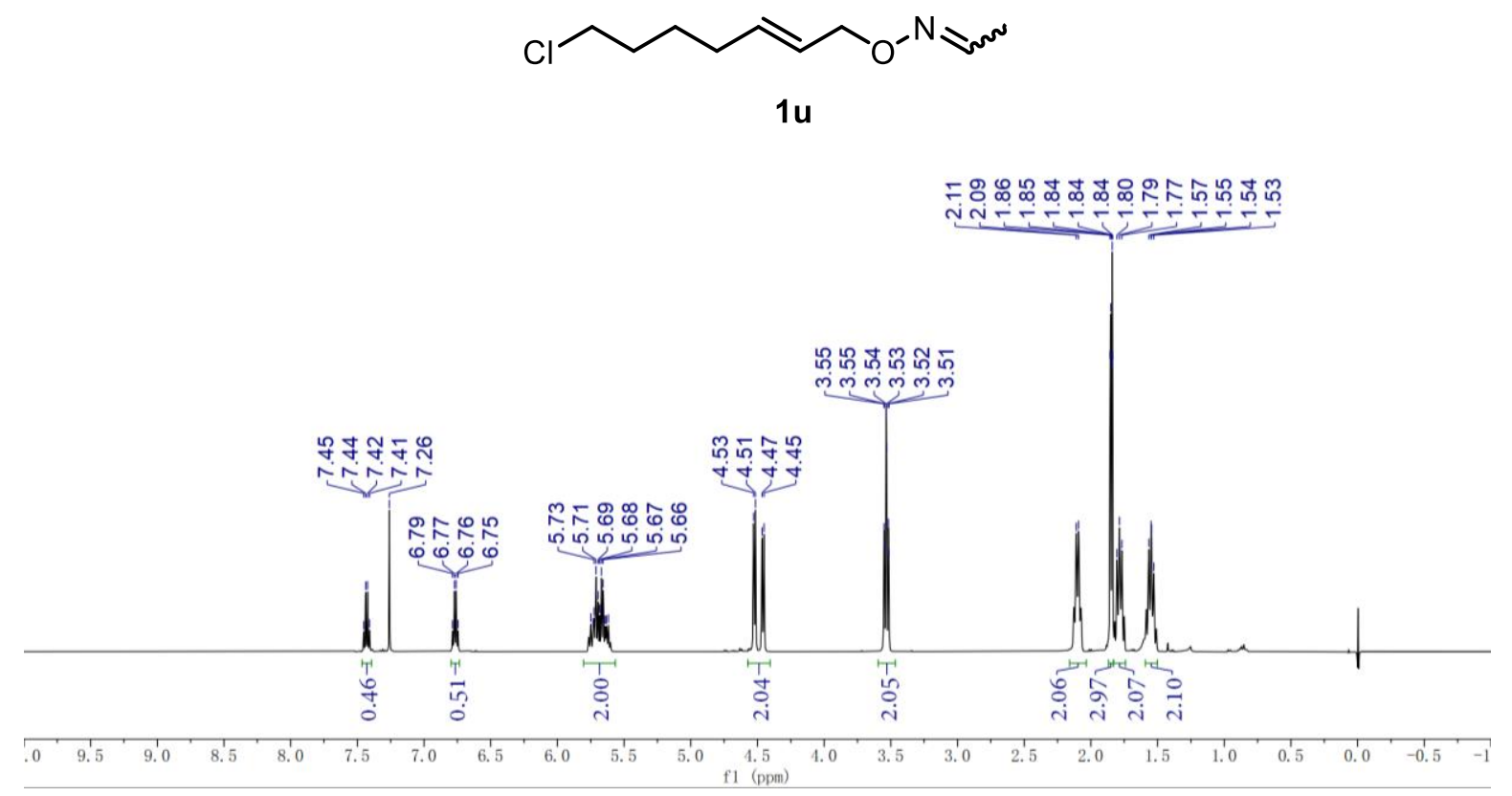

${ }^{1} \mathrm{H}$ NMR spectrum of $\mathbf{1} \mathbf{u}$ was measured in $\mathrm{CDCl}_{3}$ at $400 \mathrm{MH}$
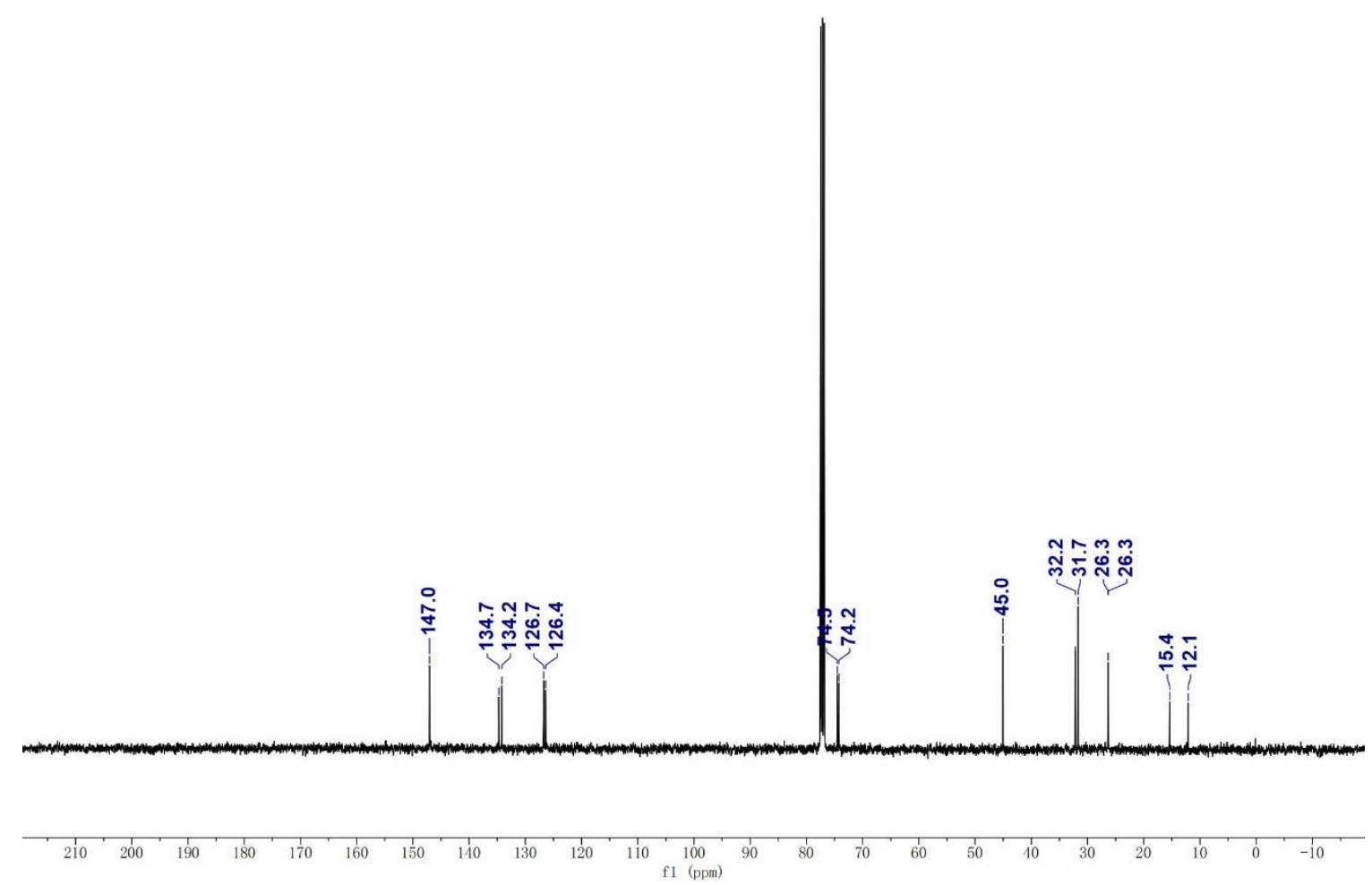

${ }^{13} \mathrm{C}$ NMR spectrum of $1 \mathbf{u}$ was measured in $\mathrm{CDCl}_{3}$ at $101 \mathrm{MHz}$ 

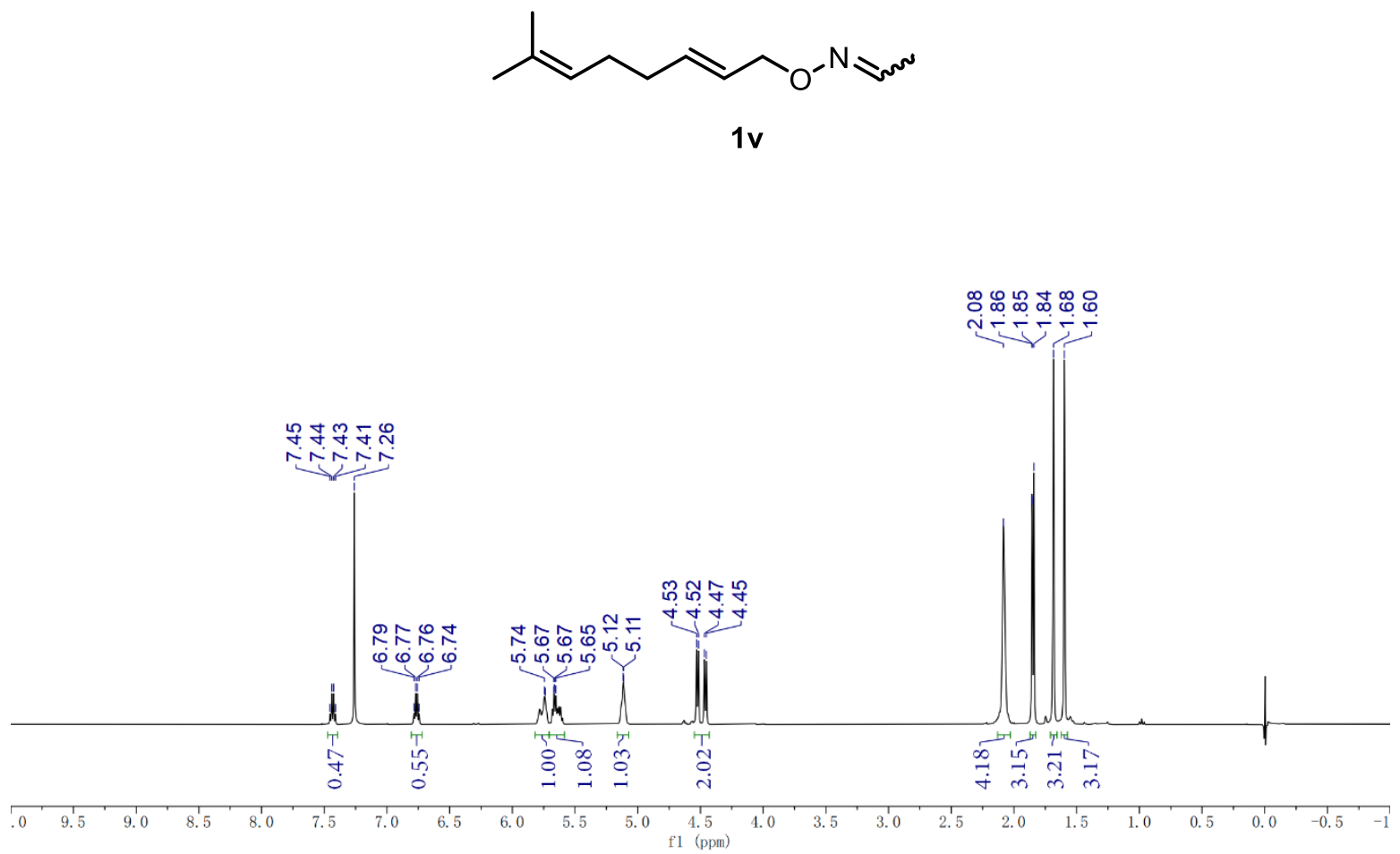

${ }^{1} \mathrm{H}$ NMR spectrum of $\mathbf{1 v}$ was measured in $\mathrm{CDCl}_{3}$ at $400 \mathrm{MH}$
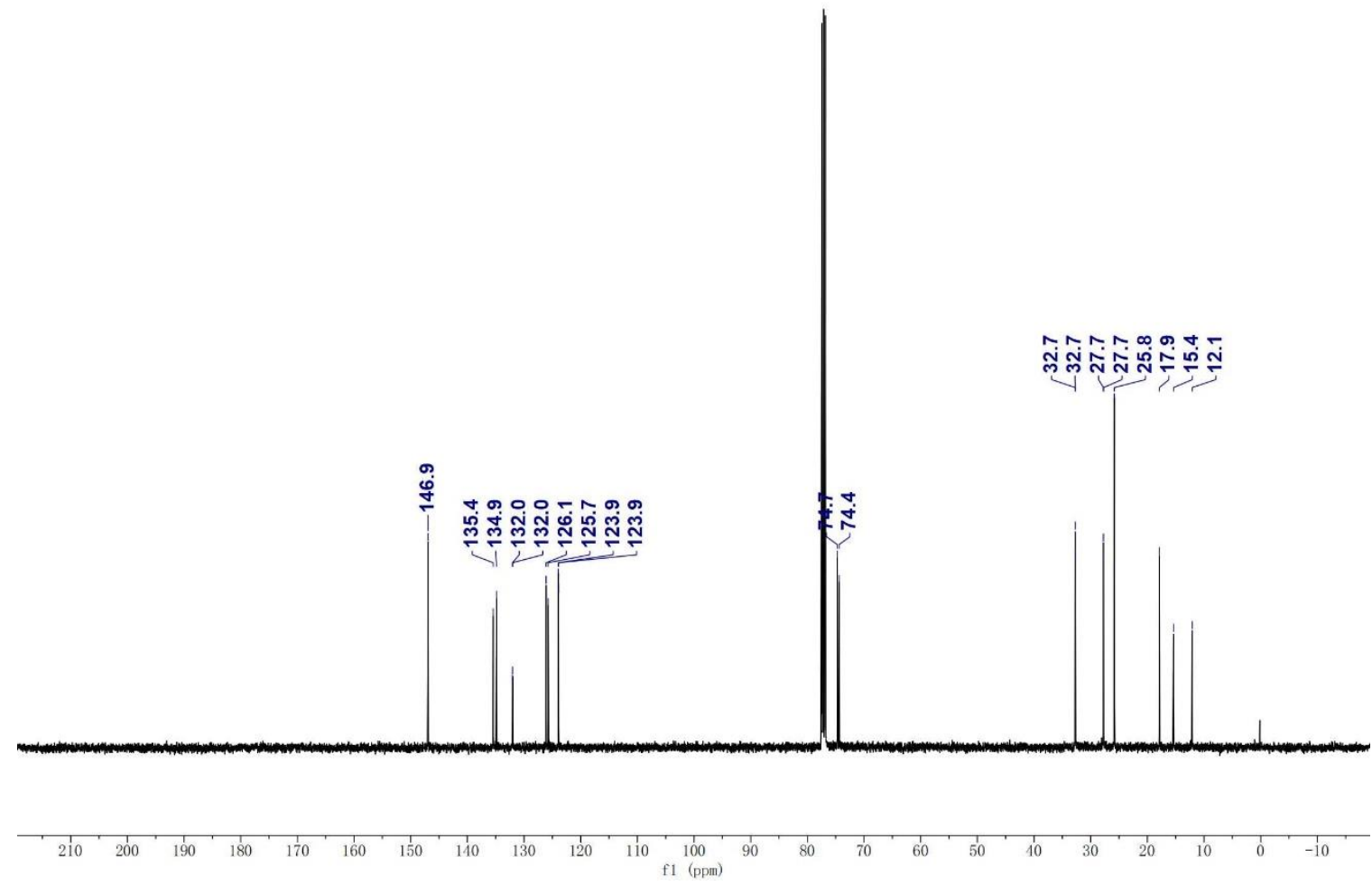

${ }^{13} \mathrm{C}$ NMR spectrum of $\mathbf{1 v}$ was measured in $\mathrm{CDCl}_{3}$ at $101 \mathrm{MHz}$ 


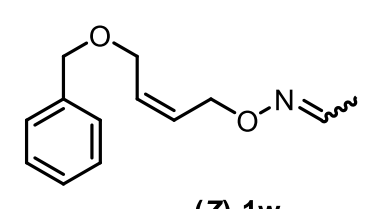

(Z)-1w

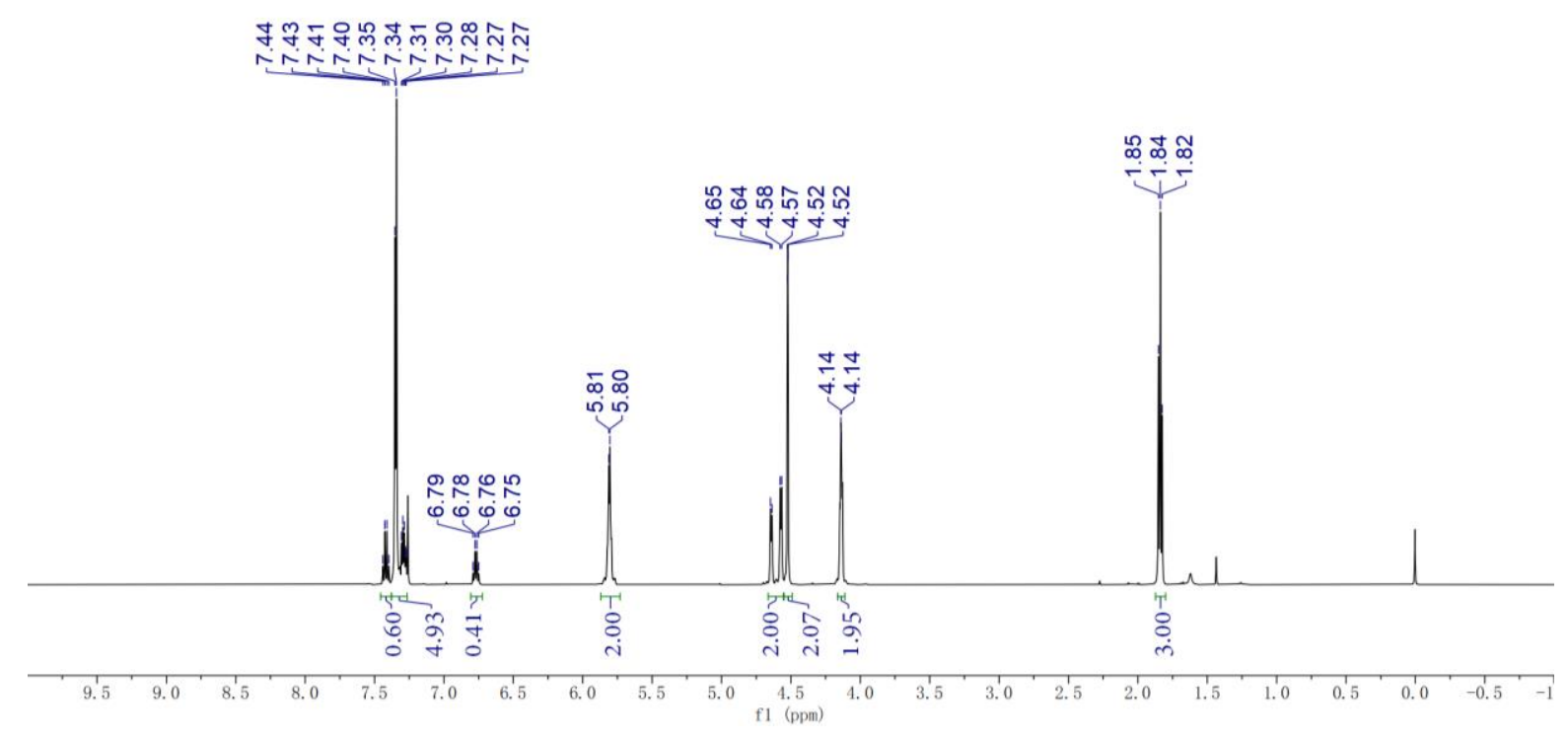

${ }^{1} \mathrm{H}$ NMR spectrum of $(\boldsymbol{Z})-\mathbf{1} \mathbf{w}$ was measured in $\mathrm{CDCl}_{3}$ at $400 \mathrm{MH}$
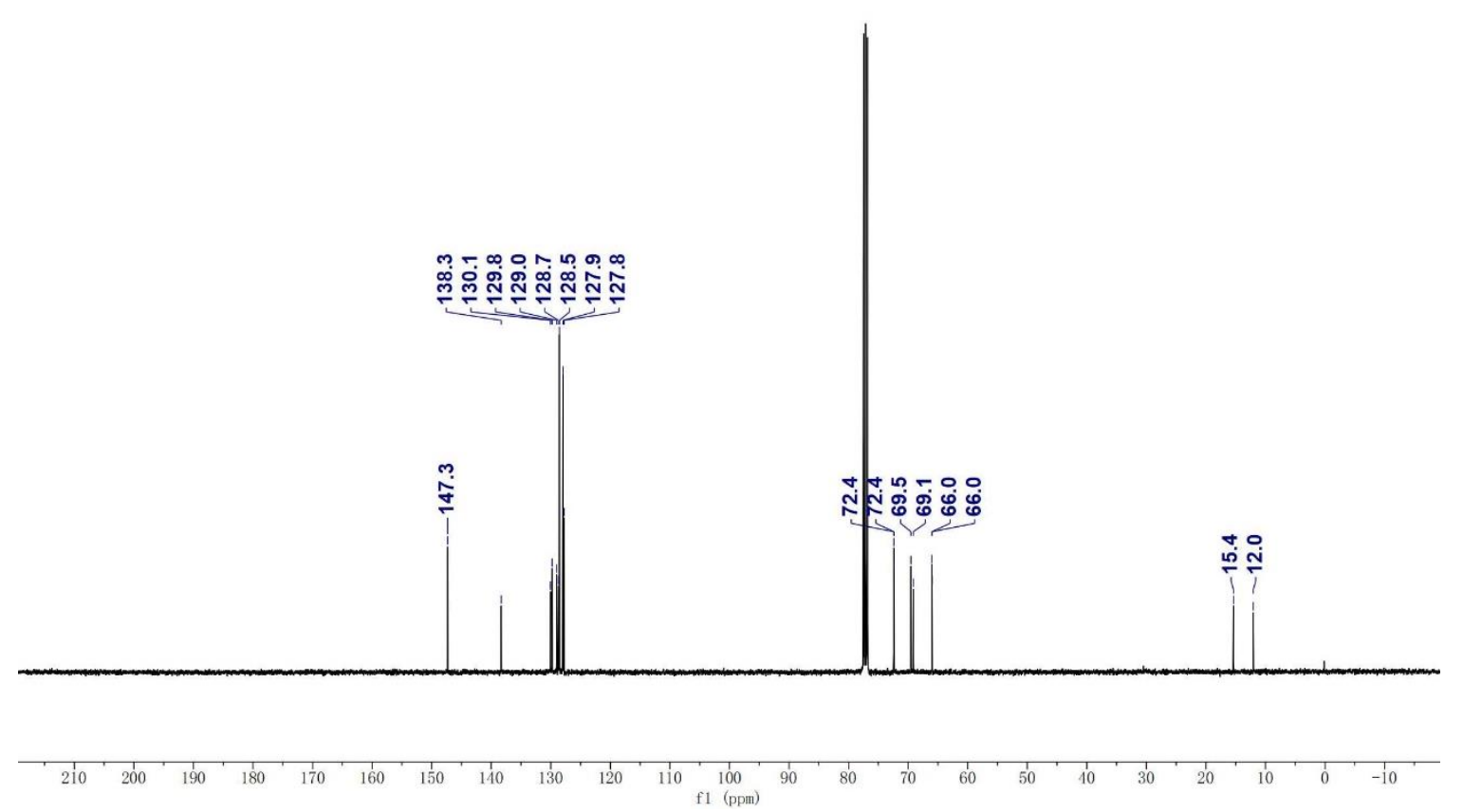

${ }^{13} \mathrm{C}$ NMR spectrum of $(Z)-\mathbf{1} \mathbf{w}$ was measured in $\mathrm{CDCl}_{3}$ at $101 \mathrm{MHz}$ 


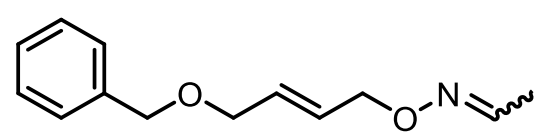

(E)-1W

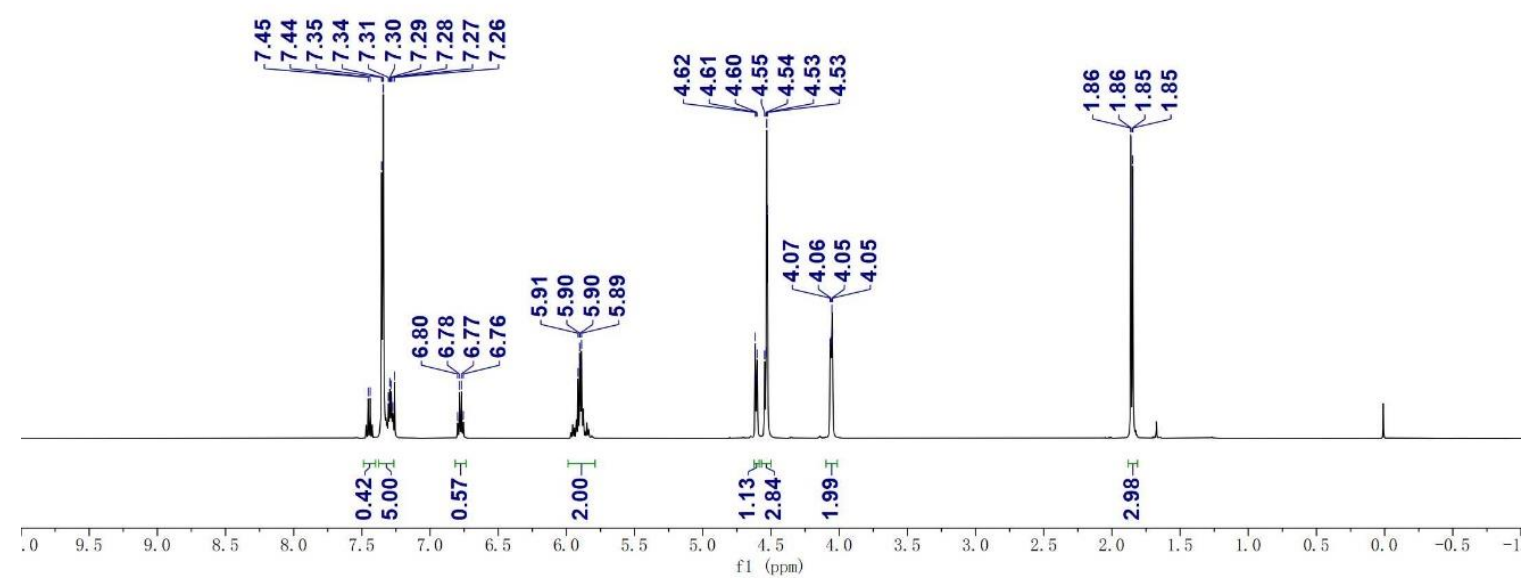

${ }^{1} \mathrm{H}$ NMR spectrum of $(\boldsymbol{E})-\mathbf{1 w}$ was measured in $\mathrm{CDCl}_{3}$ at $400 \mathrm{MH}$
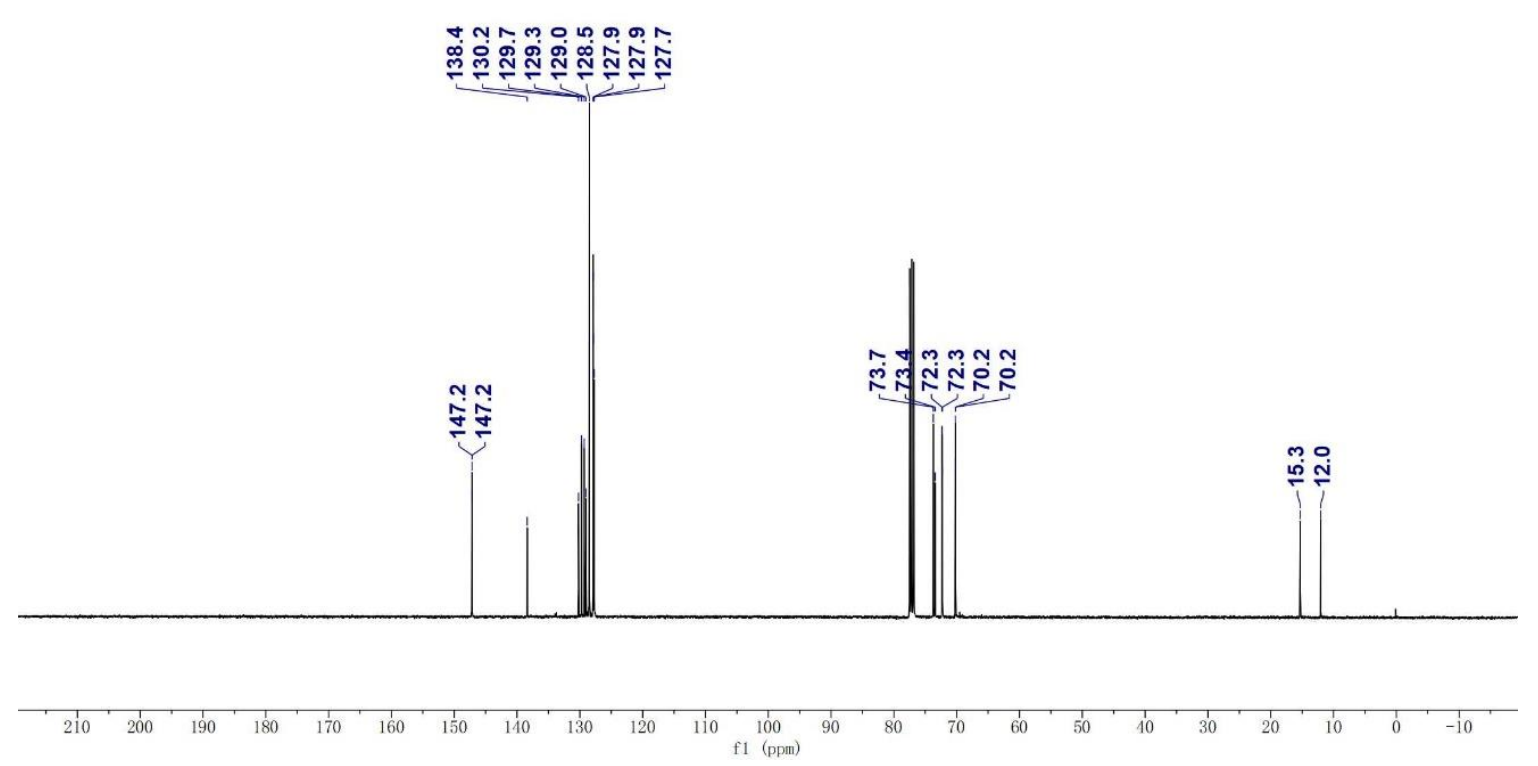

${ }^{13} \mathrm{C}$ NMR spectrum of $(\boldsymbol{E})-\mathbf{1} \mathbf{w}$ was measured in $\mathrm{CDCl}_{3}$ at $101 \mathrm{MHz}$ 


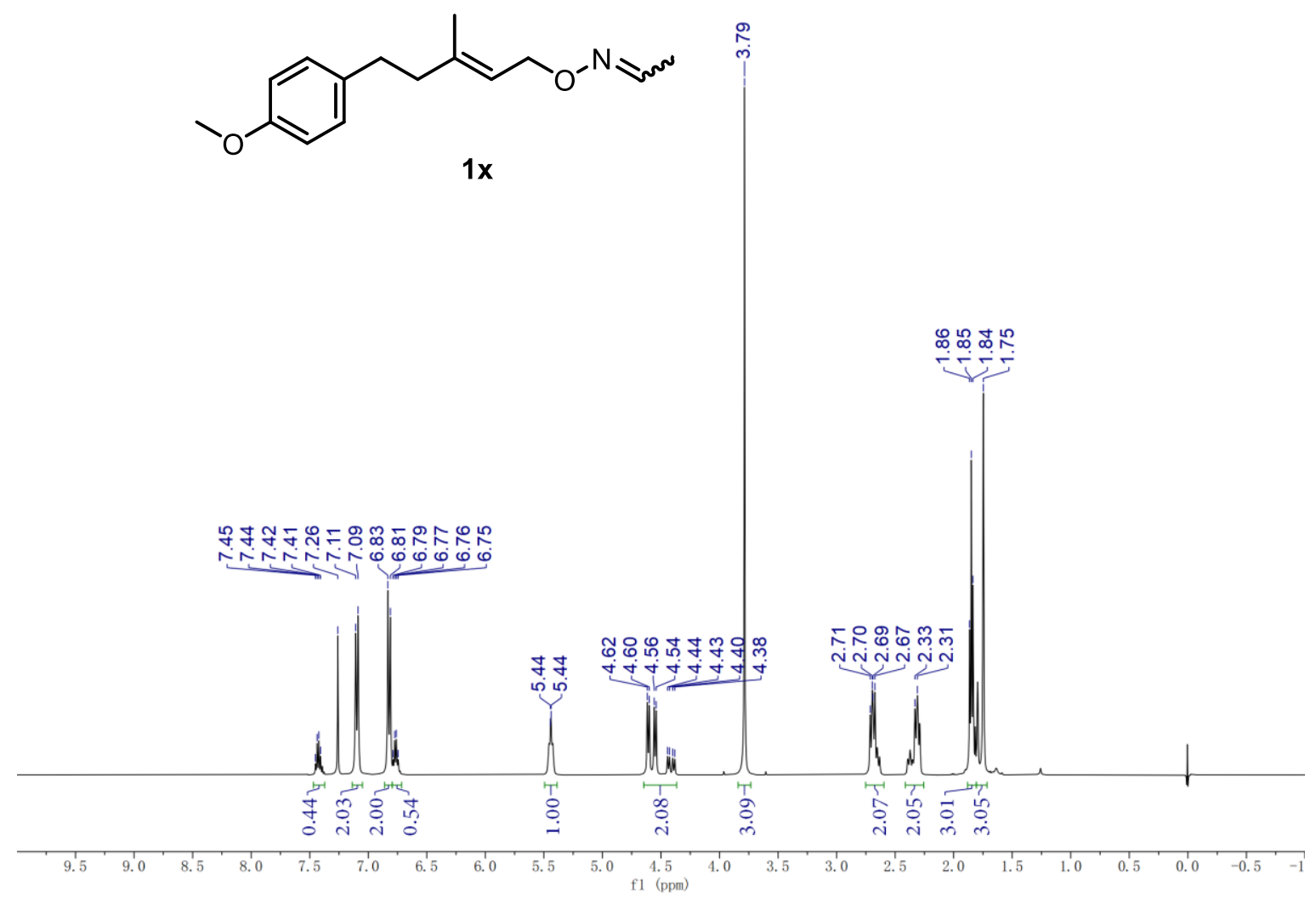

${ }^{1} \mathrm{H}$ NMR spectrum of $\mathbf{1 x}$ was measured in $\mathrm{CDCl}_{3}$ at $400 \mathrm{MH}$
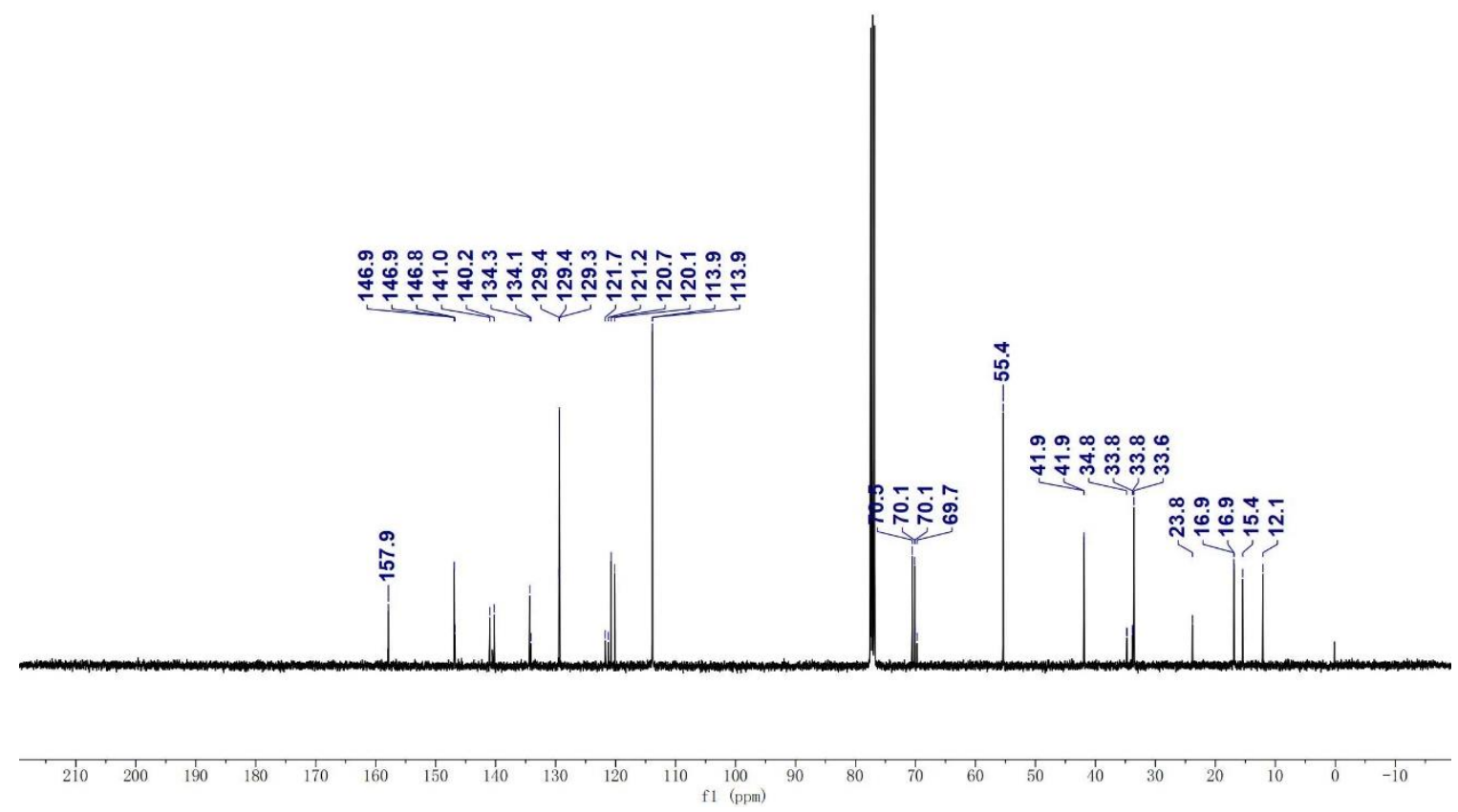

${ }^{13} \mathrm{C}$ NMR spectrum of $\mathbf{1 x}$ was measured in $\mathrm{CDCl}_{3}$ at $101 \mathrm{MHz}$ 


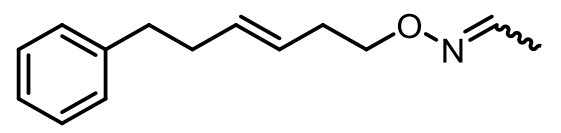

$1 y$

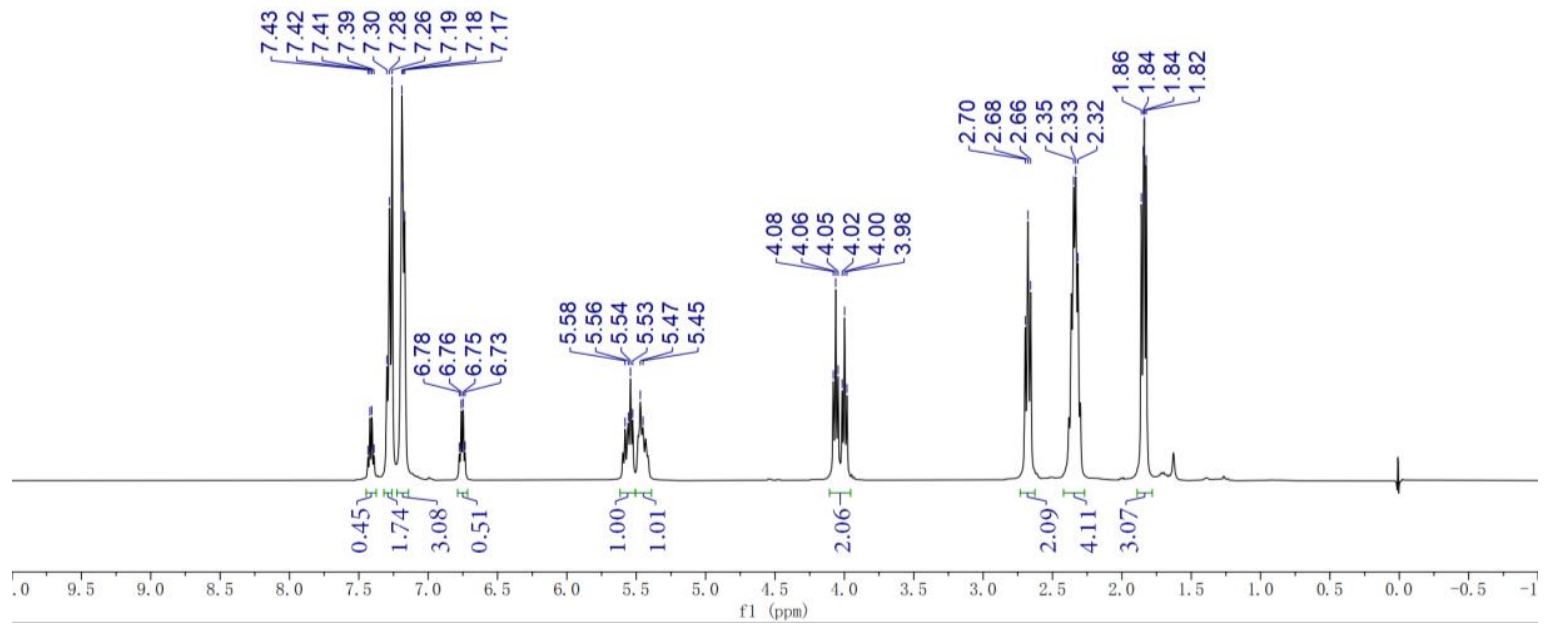

${ }^{1} \mathrm{H}$ NMR spectrum of $\mathbf{1 y}$ was measured in $\mathrm{CDCl}_{3}$ at $400 \mathrm{MH}$
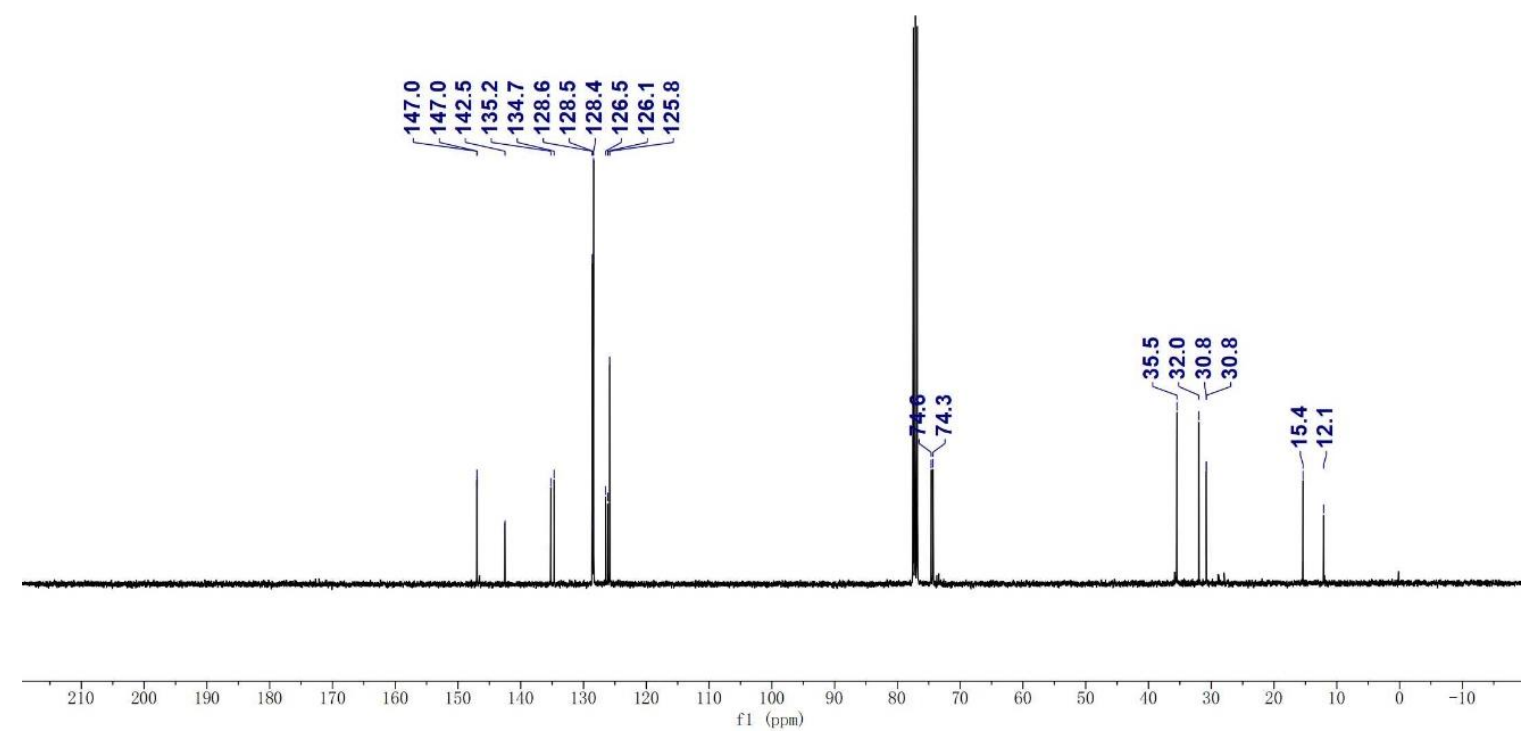

${ }^{13} \mathrm{C}$ NMR spectrum of $\mathbf{1 y}$ was measured in $\mathrm{CDCl}_{3}$ at $101 \mathrm{MHz}$ 

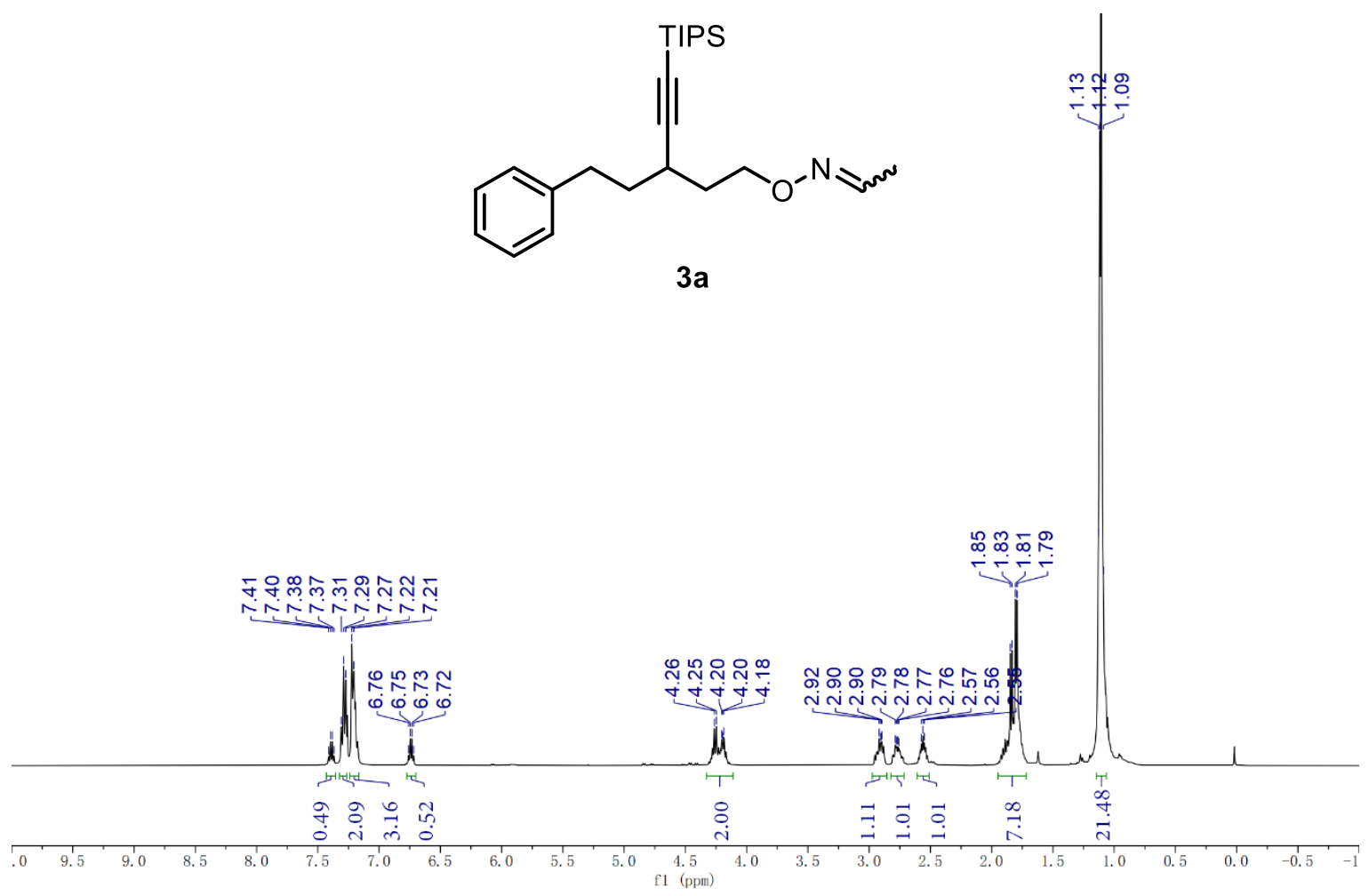

${ }^{1} \mathrm{H}$ NMR spectrum of $\mathbf{3 a}$ was measured in $\mathrm{CDCl}_{3}$ at $400 \mathrm{MH}$
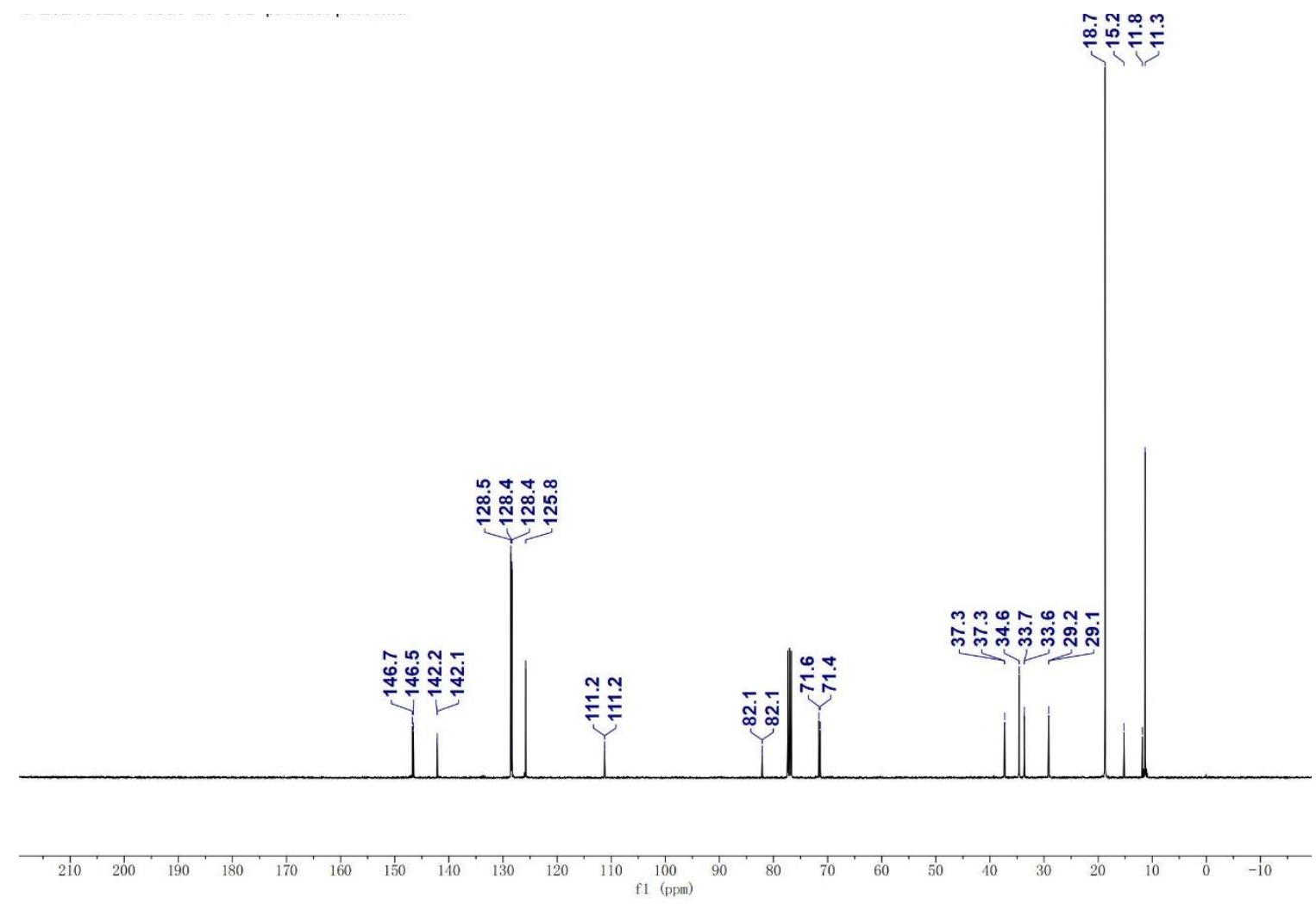

${ }^{13} \mathrm{C}$ NMR spectrum of 3a was measured in $\mathrm{CDCl}_{3}$ at $101 \mathrm{MHz}$ 


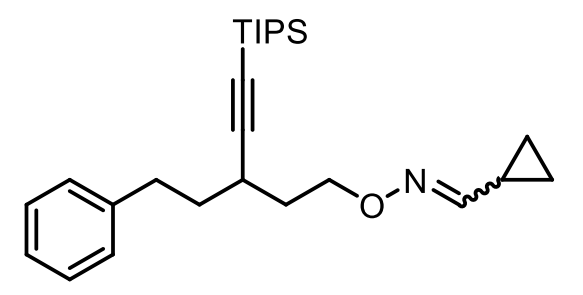

3b

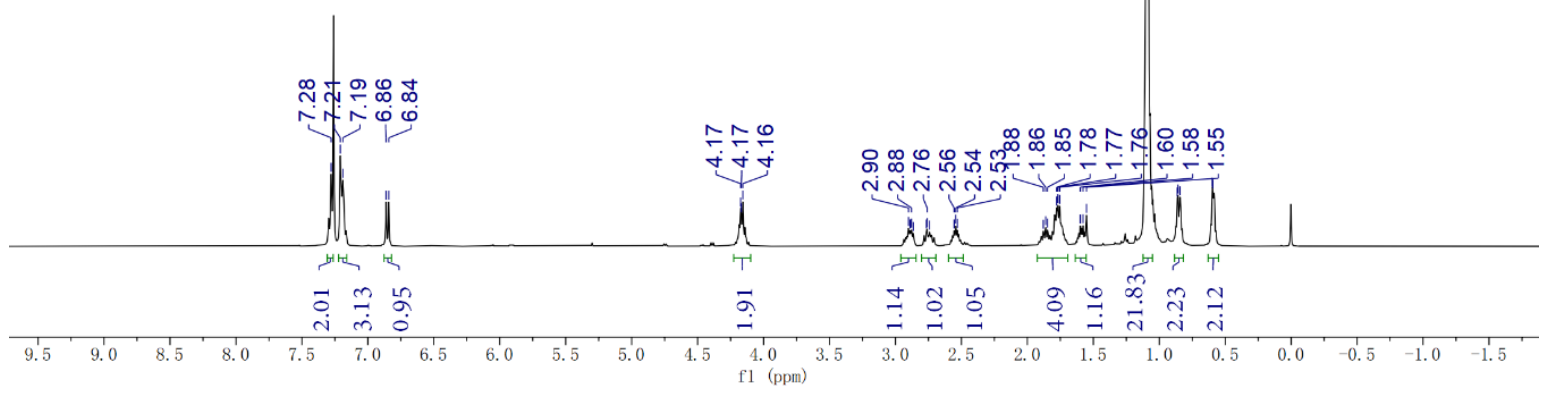

${ }^{1} \mathrm{H}$ NMR spectrum of $\mathbf{3 b}$ was measured in $\mathrm{CDCl}_{3}$ at $400 \mathrm{MH}$
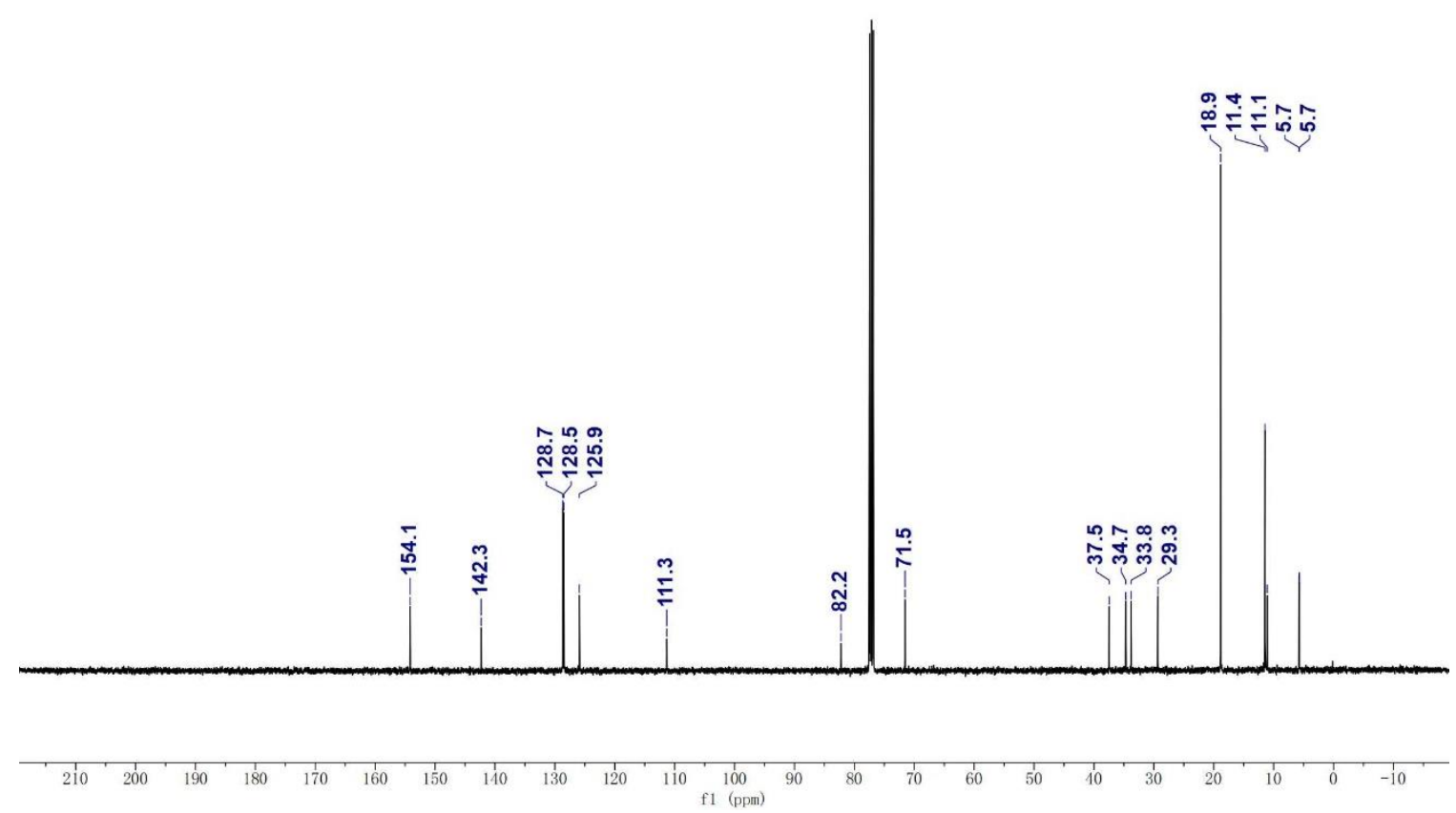

${ }^{13} \mathrm{C}$ NMR spectrum of $\mathbf{3 b}$ was measured in $\mathrm{CDCl}_{3}$ at $101 \mathrm{MHz}$ 

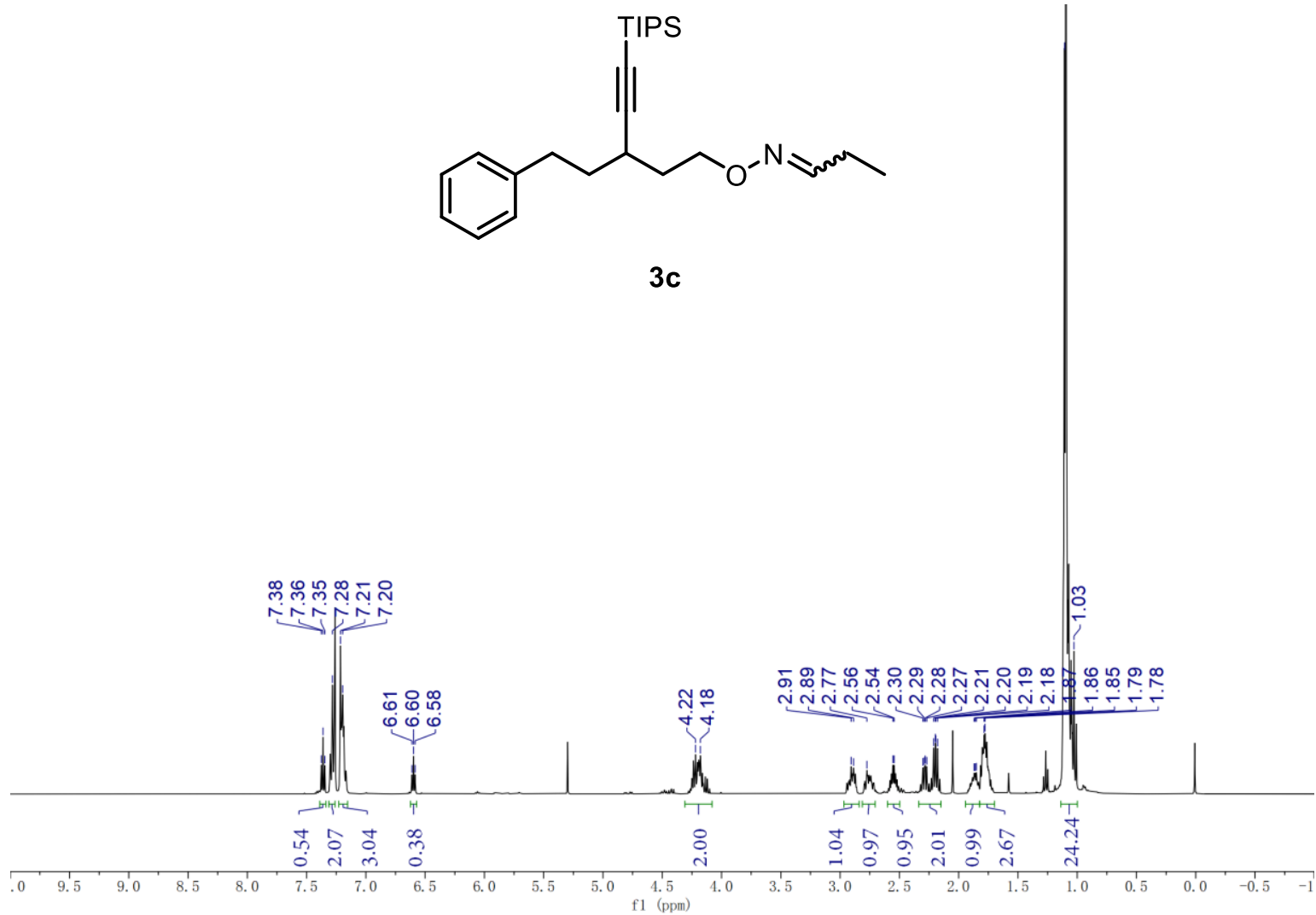

${ }^{1} \mathrm{H}$ NMR spectrum of $3 \mathbf{c}$ was measured in $\mathrm{CDCl}_{3}$ at $400 \mathrm{MH}$
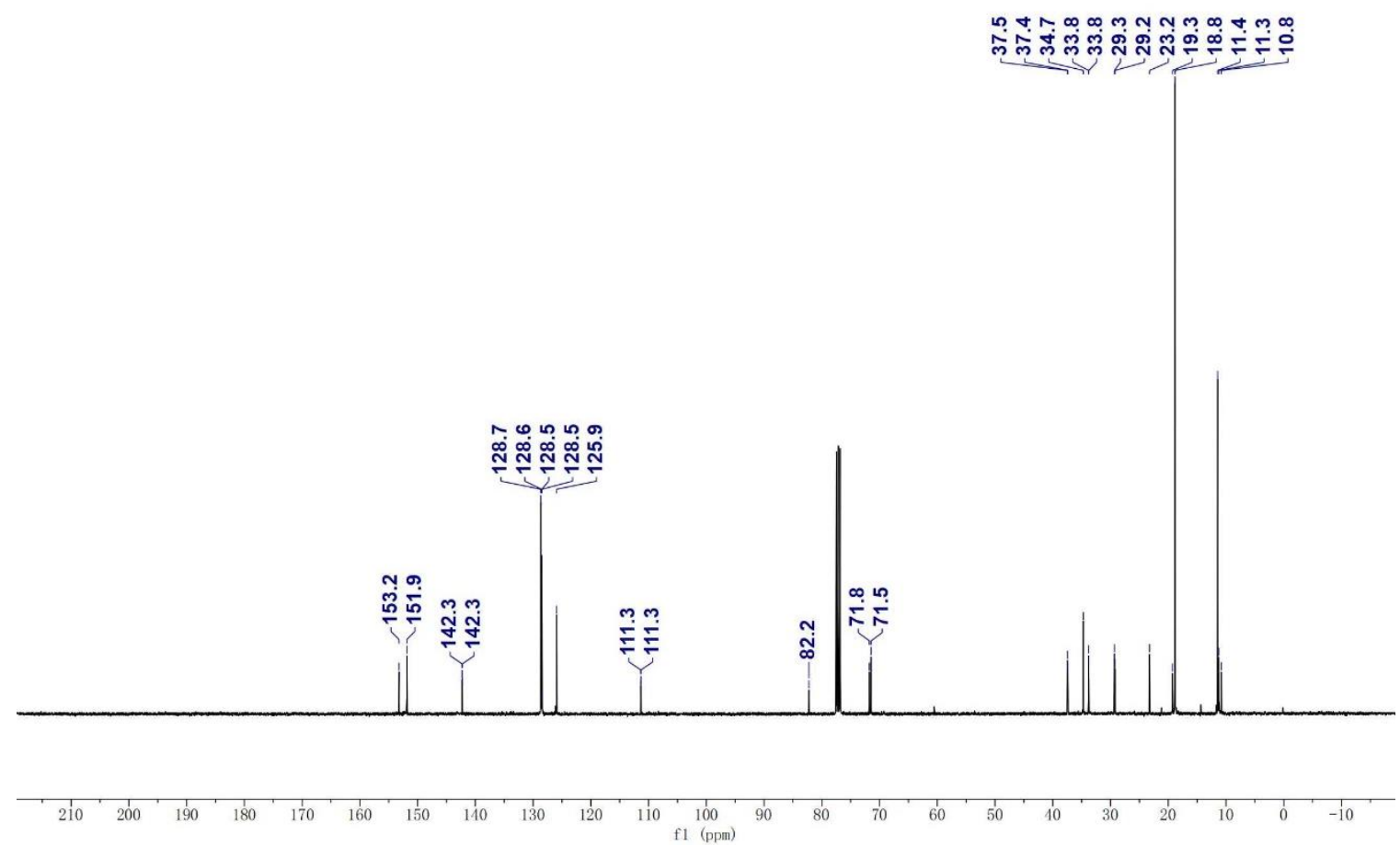

${ }^{13} \mathrm{C}$ NMR spectrum of $3 \mathbf{c}$ was measured in $\mathrm{CDCl}_{3}$ at $101 \mathrm{MHz}$ 

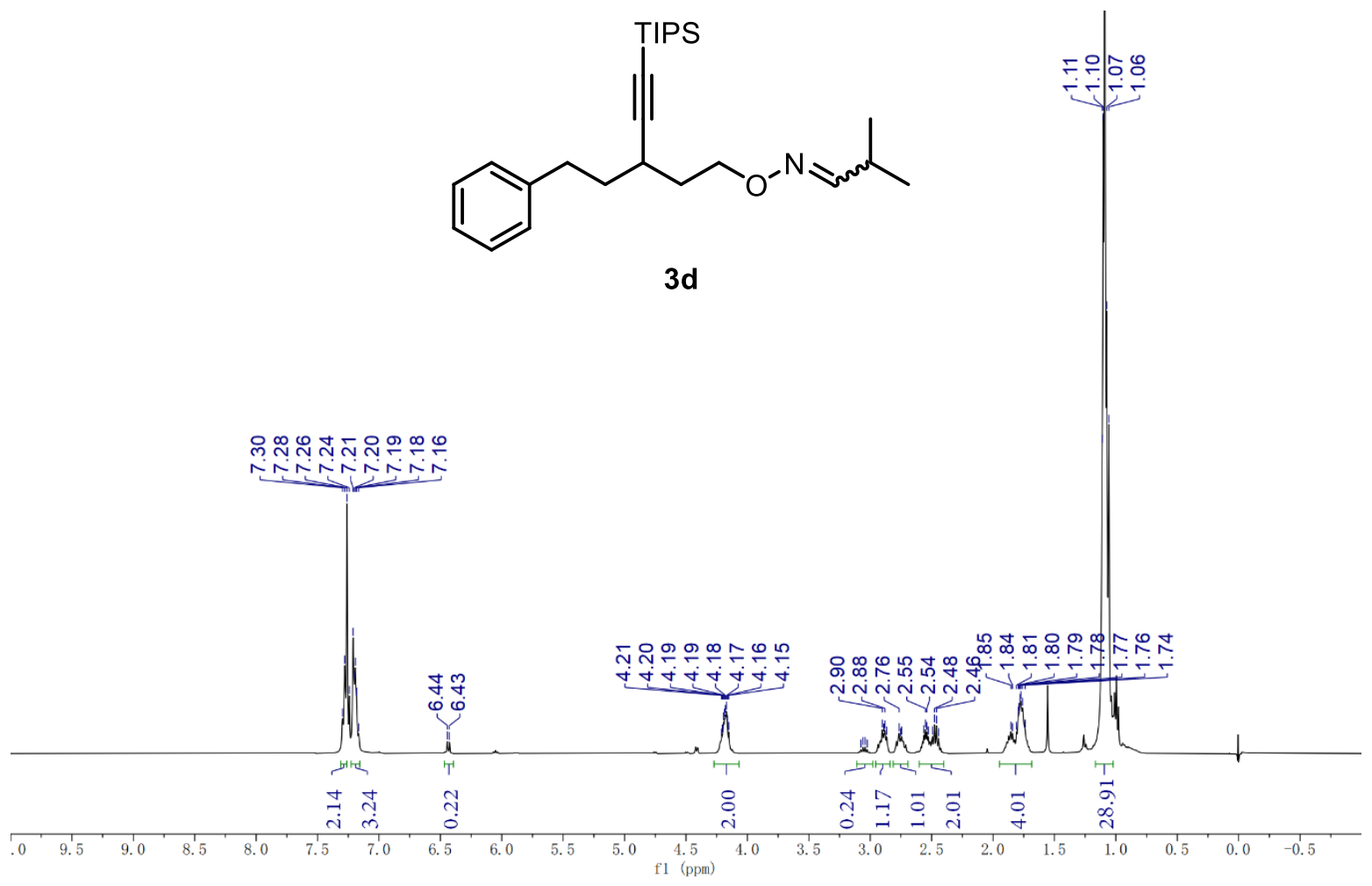

${ }^{1} \mathrm{H}$ NMR spectrum of $\mathbf{3 d}$ was measured in $\mathrm{CDCl}_{3}$ at $400 \mathrm{MH}$
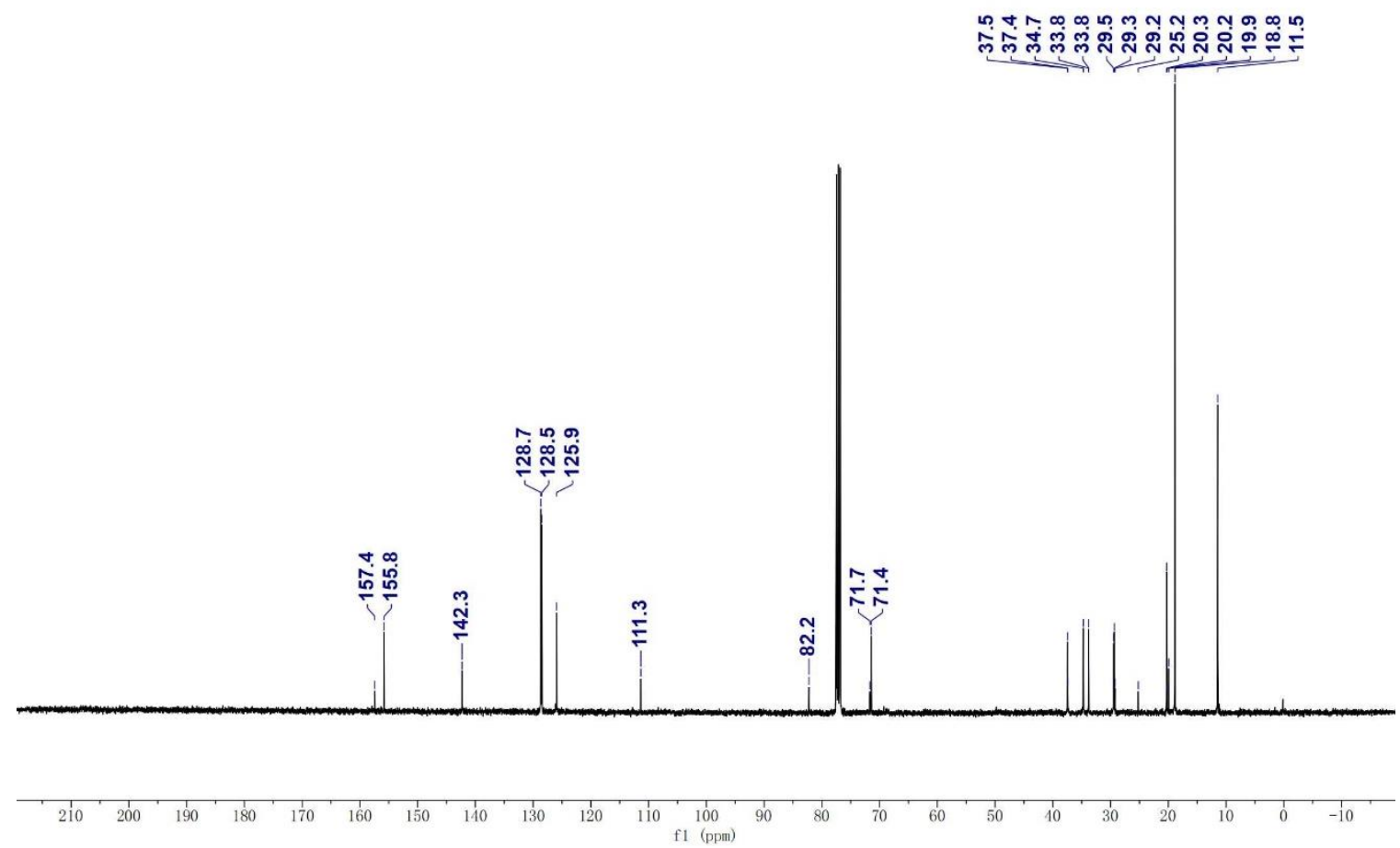

${ }^{13} \mathrm{C}$ NMR spectrum of $\mathbf{3 d}$ was measured in $\mathrm{CDCl}_{3}$ at $101 \mathrm{MHz}$ 

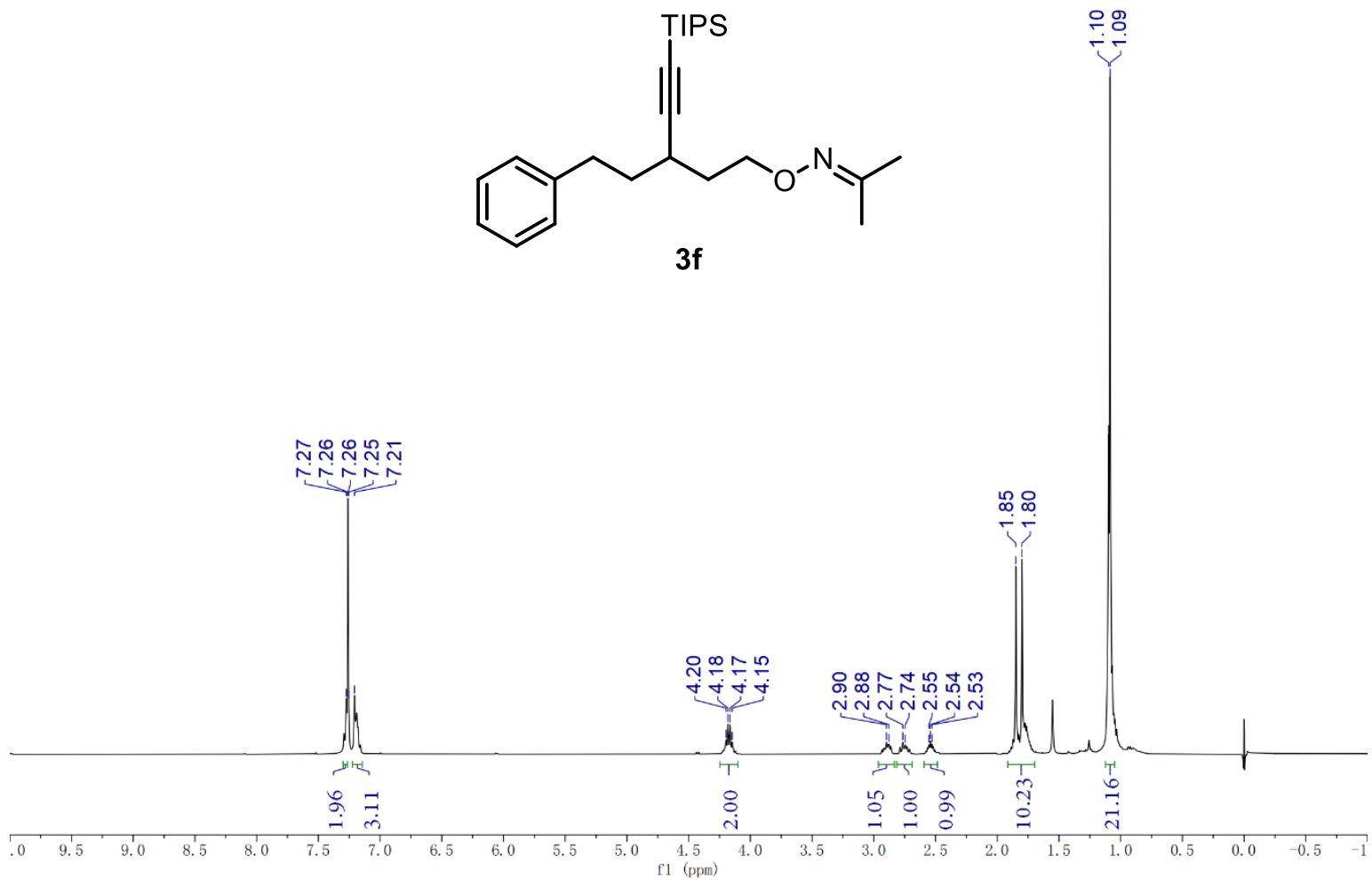

${ }^{1} \mathrm{H}$ NMR spectrum of $\mathbf{3 f}$ was measured in $\mathrm{CDCl}_{3}$ at $400 \mathrm{MH}$
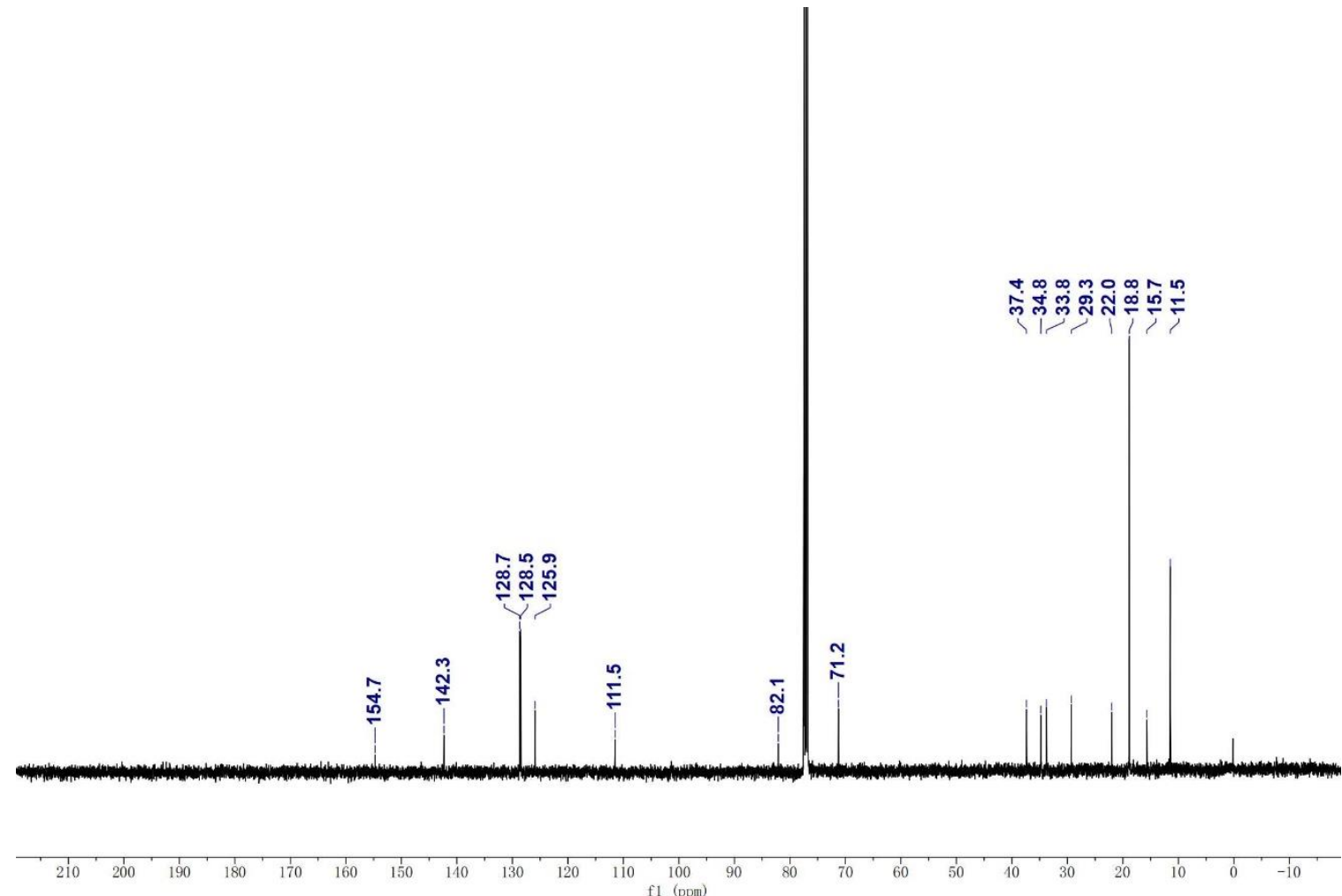

${ }^{13} \mathrm{C}$ NMR spectrum of $\mathbf{3 f}$ was measured in $\mathrm{CDCl}_{3}$ at $101 \mathrm{MHz}$ 
<smiles>COc1ccccc1C=NOCCC(C#CC(F)F)CCc1ccccc1</smiles>

$3 h$

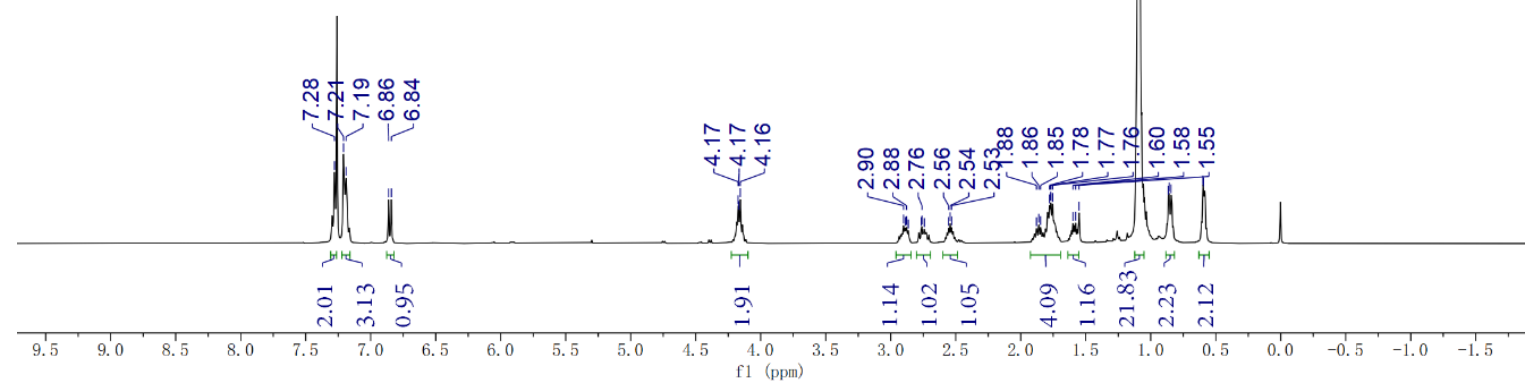

${ }^{1} \mathrm{H}$ NMR spectrum of $\mathbf{3 h}$ was measured in $\mathrm{CDCl}_{3}$ at $400 \mathrm{MH}$

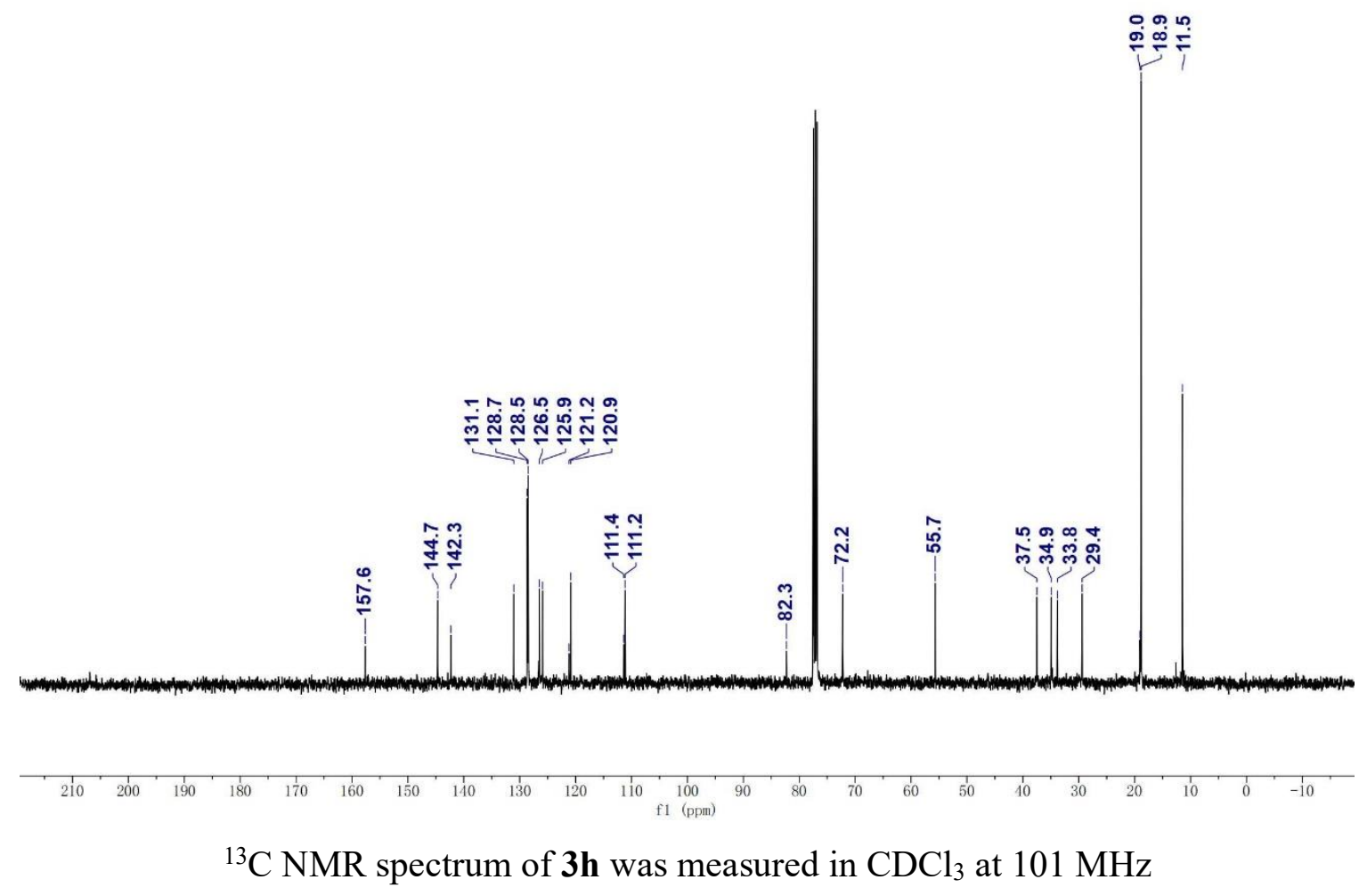




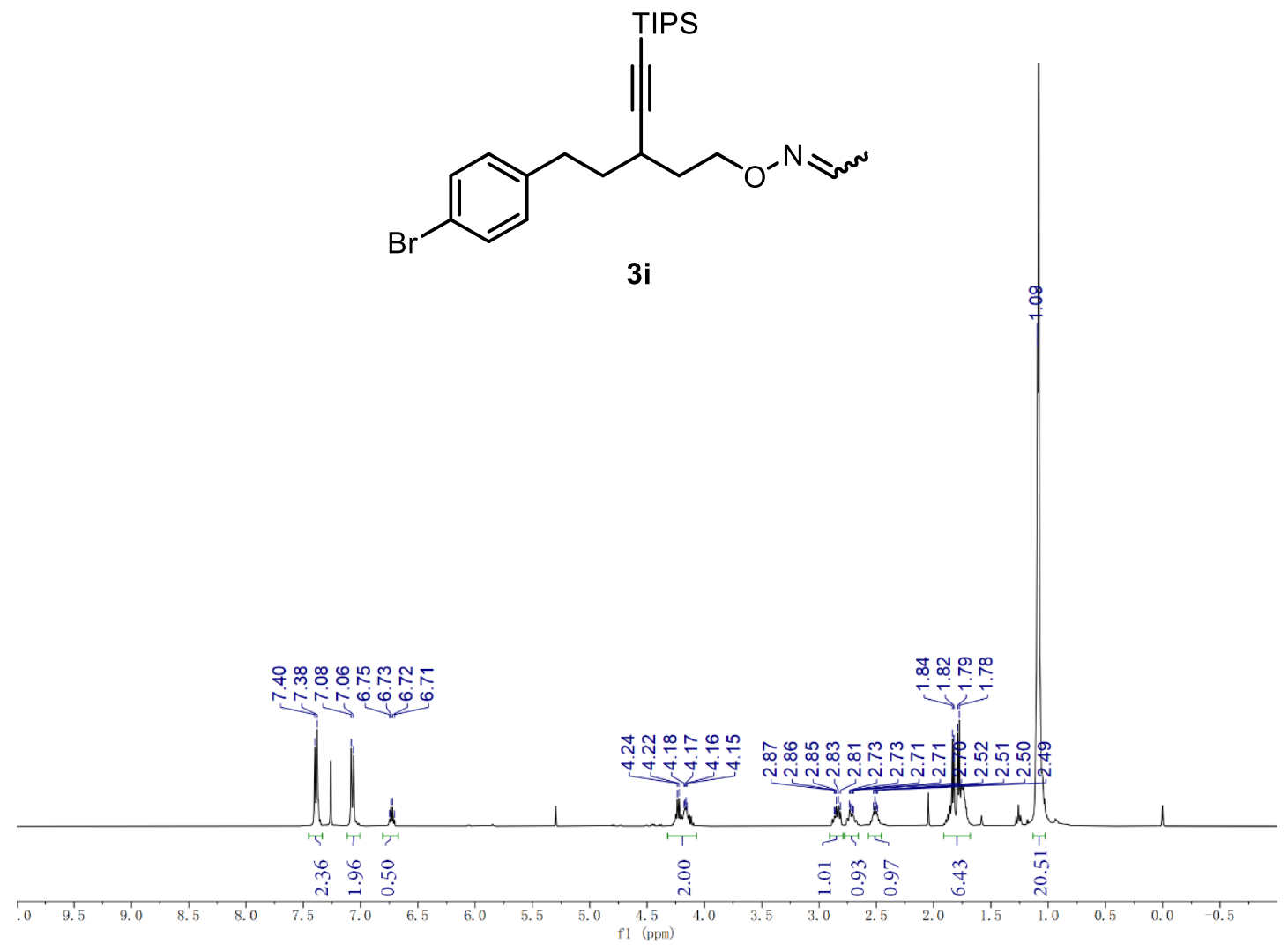

${ }^{1} \mathrm{H}$ NMR spectrum of $\mathbf{3 i}$ was measured in $\mathrm{CDCl}_{3}$ at $400 \mathrm{MH}$
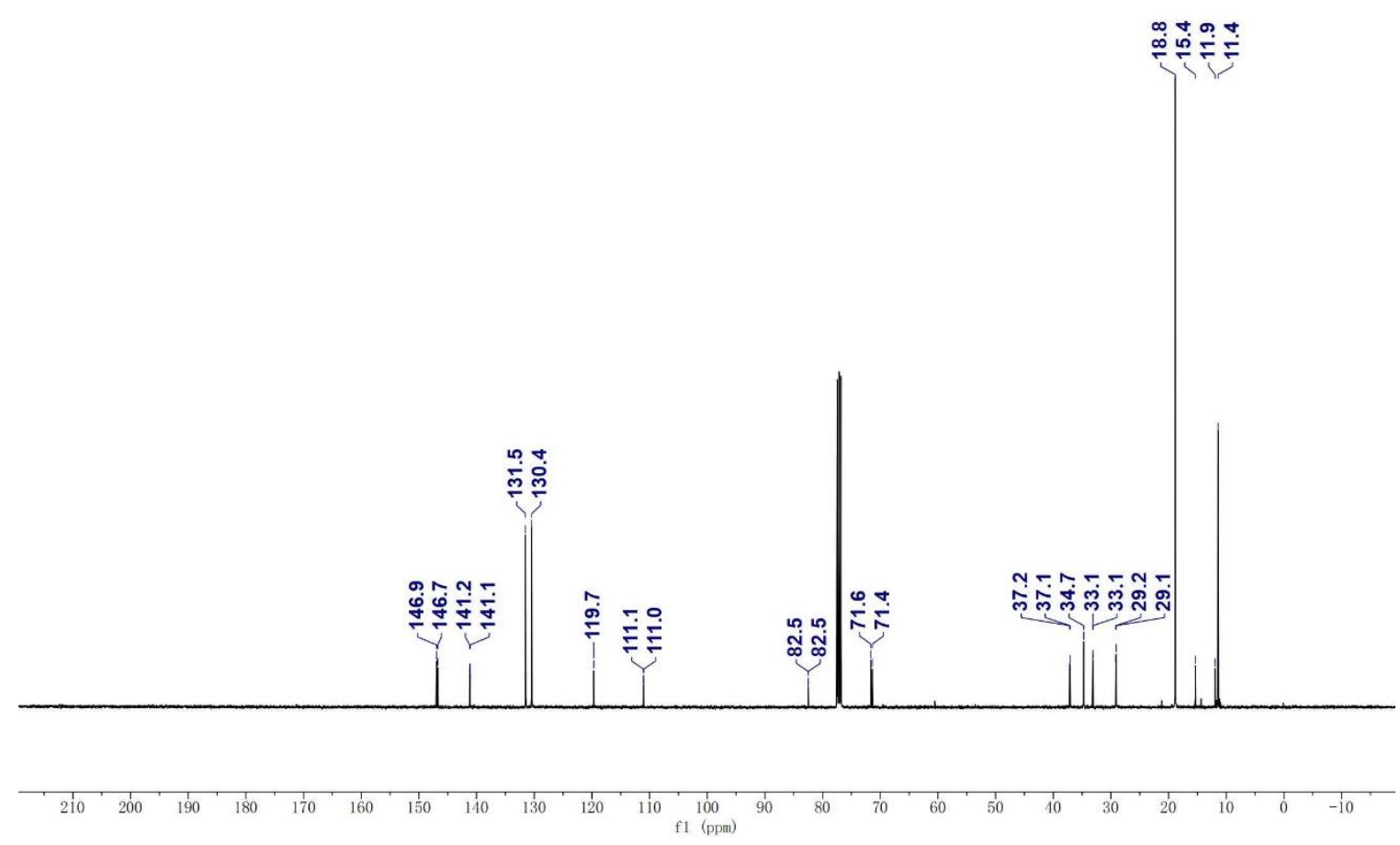

${ }^{13} \mathrm{C}$ NMR spectrum of $\mathbf{3 i}$ was measured in $\mathrm{CDCl}_{3}$ at $101 \mathrm{MHz}$ 


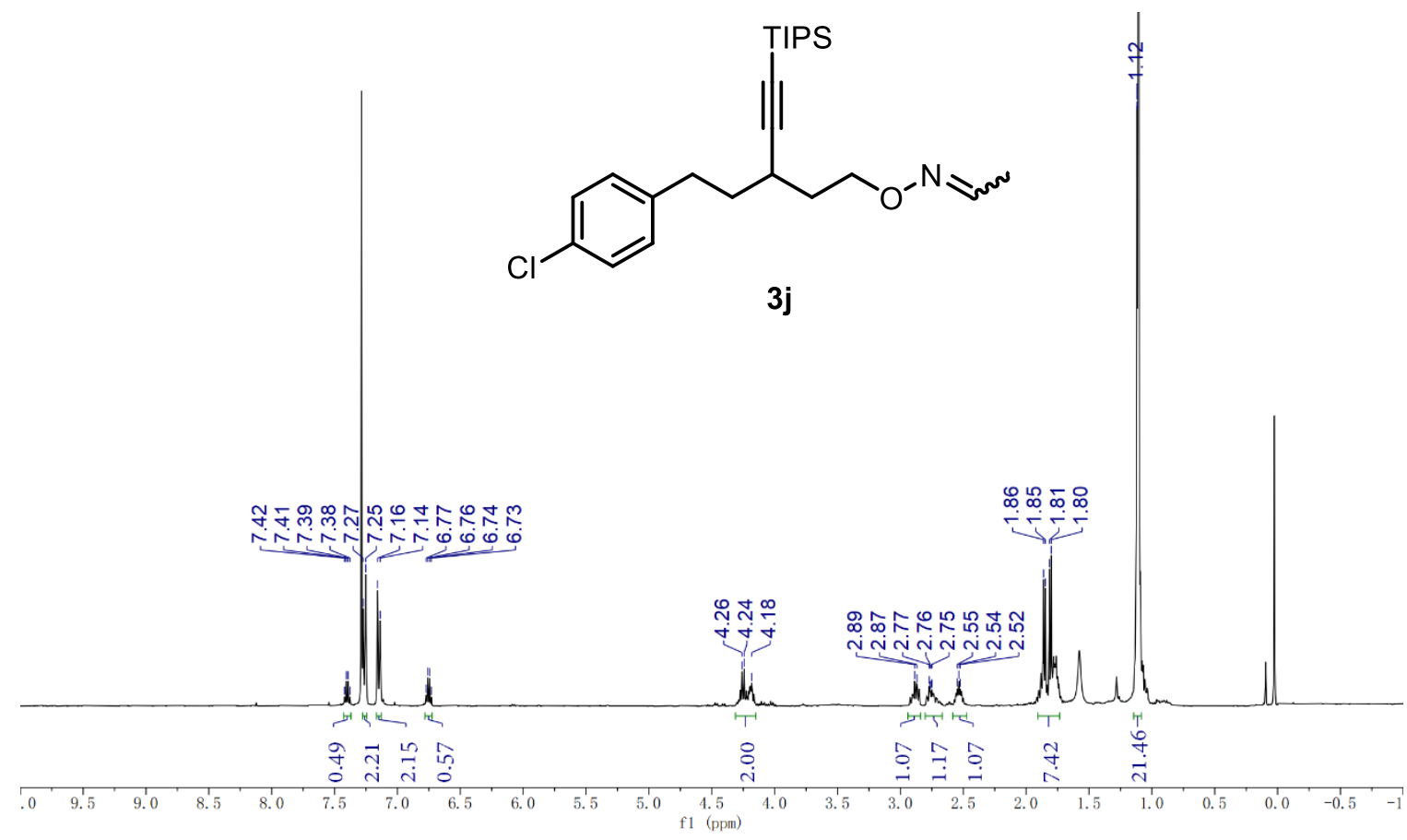

${ }^{1} \mathrm{H} \mathrm{NMR}$ spectrum of $\mathbf{3 j}$ was measured in $\mathrm{CDCl}_{3}$ at $400 \mathrm{MH}$
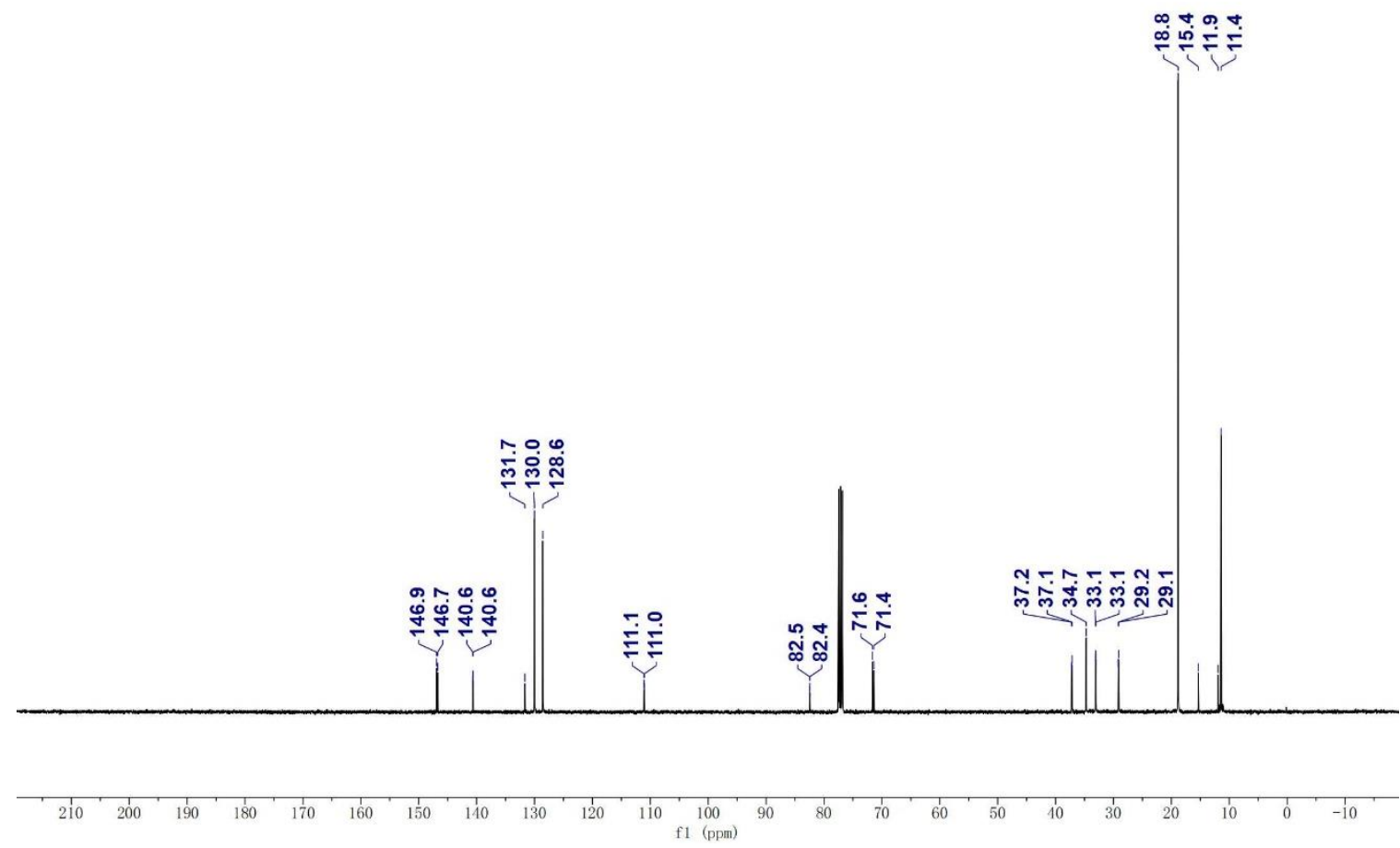

${ }^{13} \mathrm{C} \mathrm{NMR}$ spectrum of $\mathbf{3 j}$ was measured in $\mathrm{CDCl}_{3}$ at $101 \mathrm{MHz}$ 

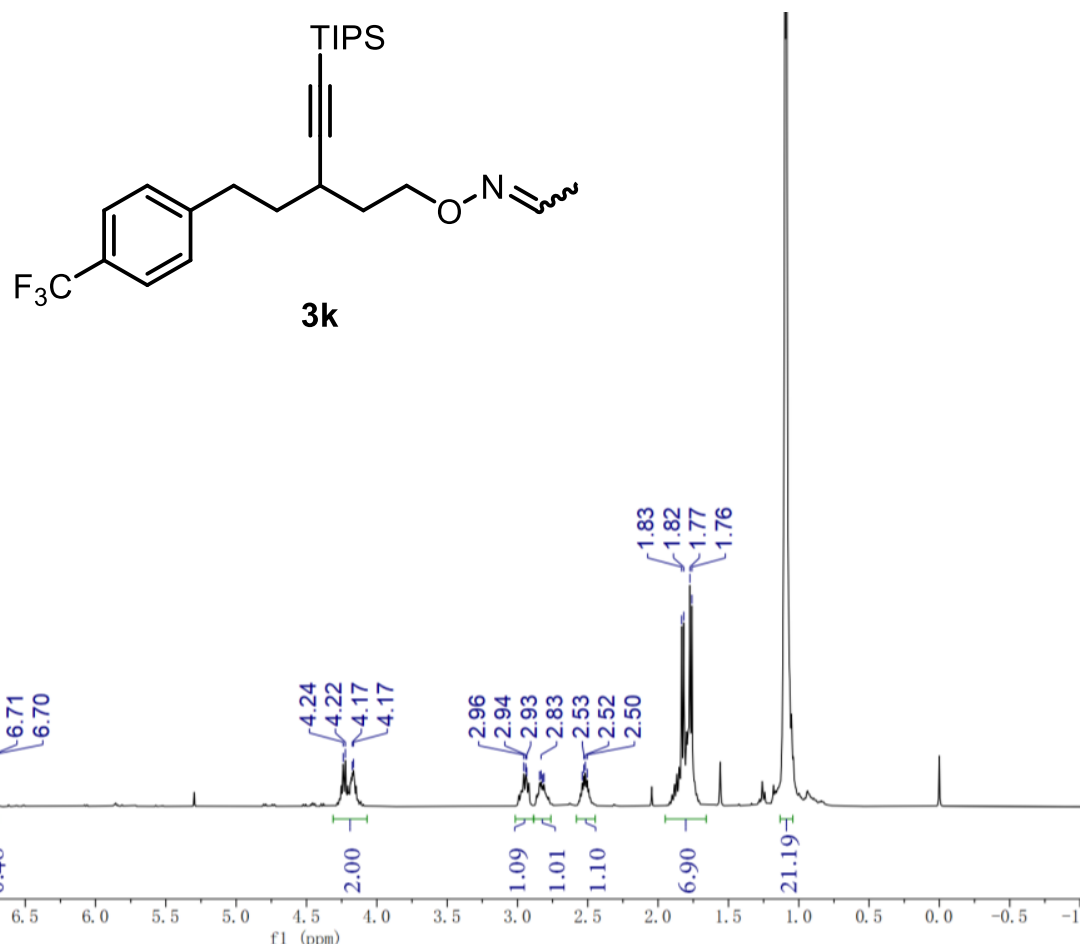

${ }^{1} \mathrm{H}$ NMR spectrum of $\mathbf{3 k}$ was measured in $\mathrm{CDCl}_{3}$ at $400 \mathrm{MH}$

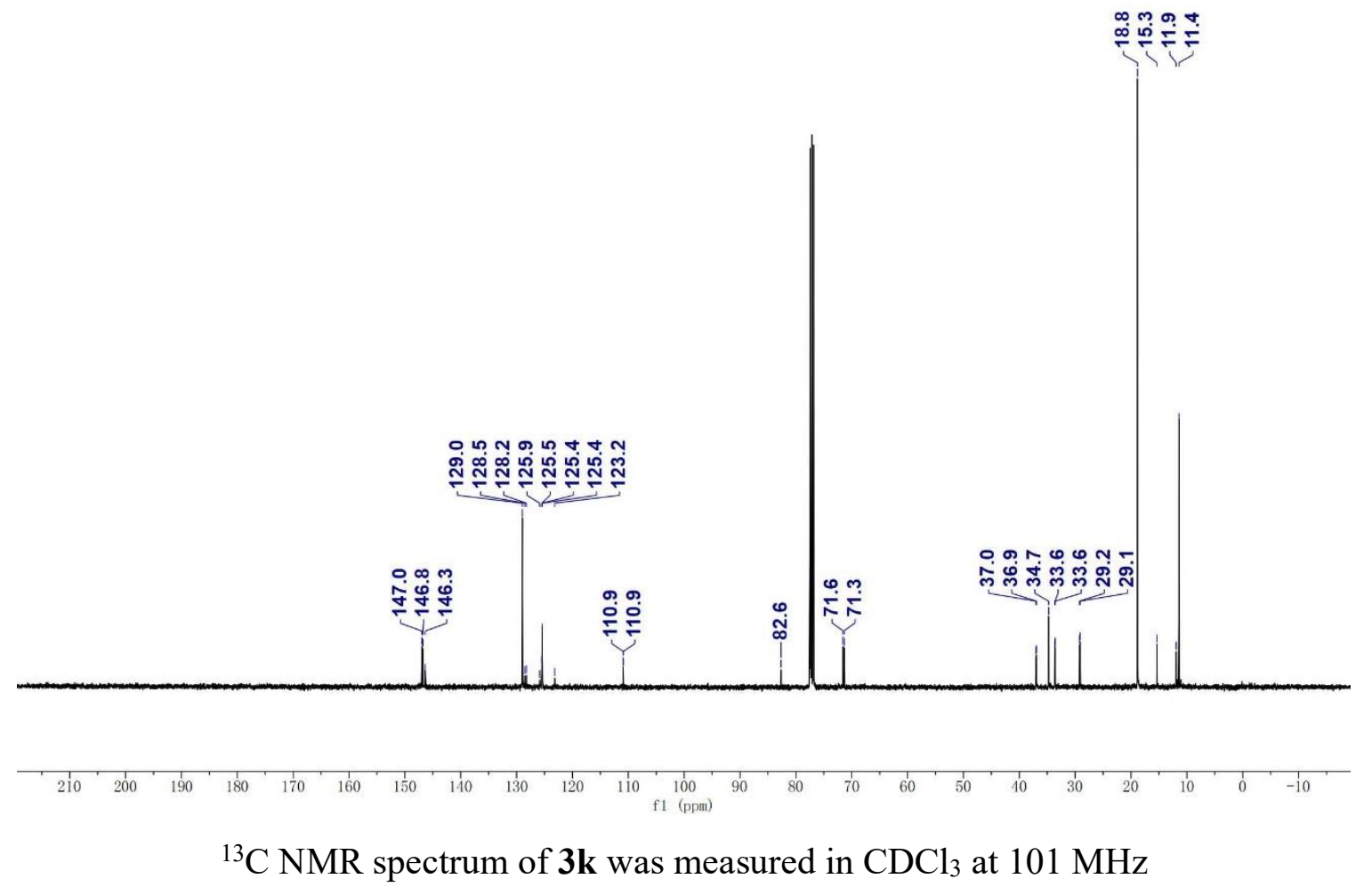




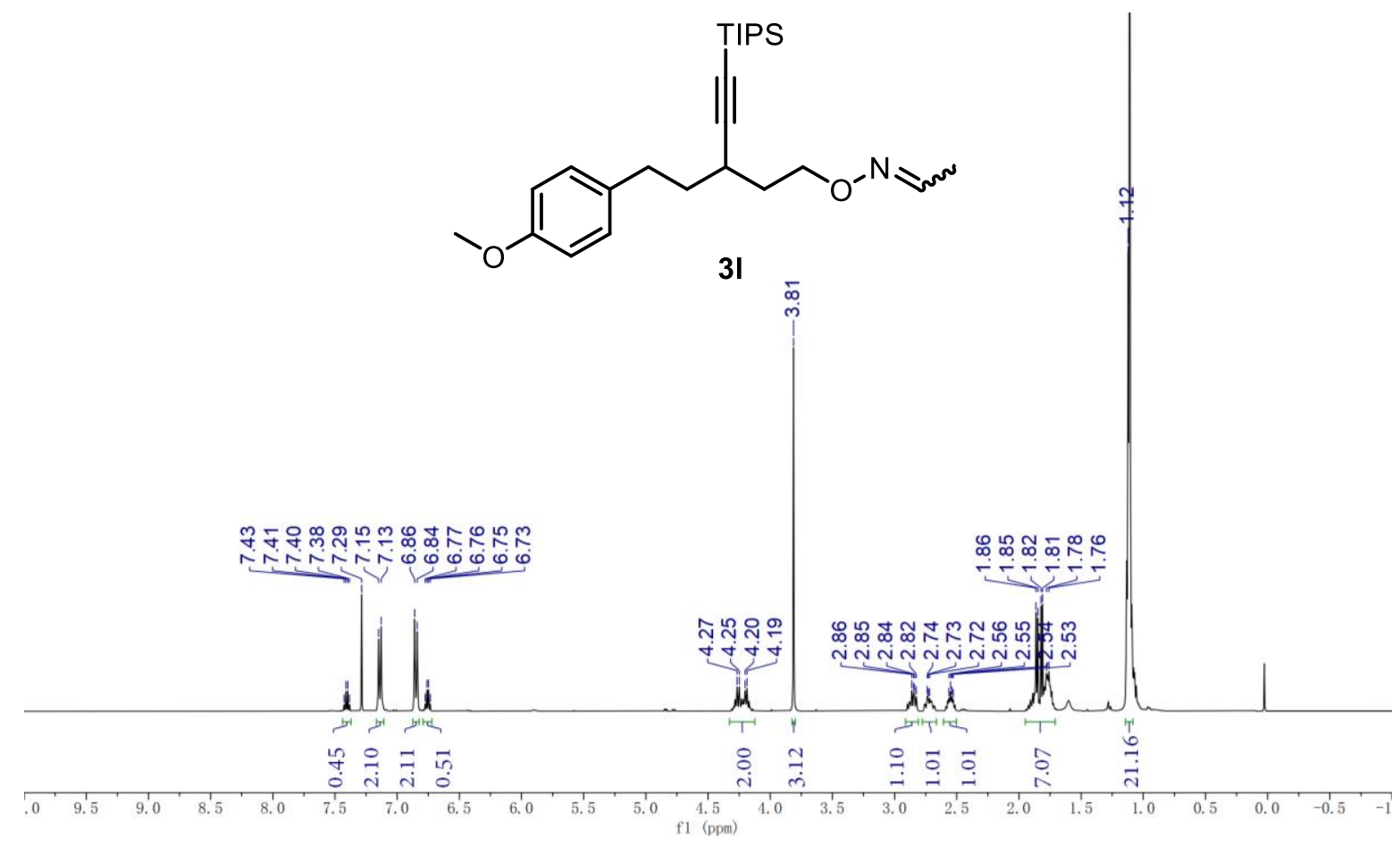

${ }^{1} \mathrm{H}$ NMR spectrum of $\mathbf{3 1}$ was measured in $\mathrm{CDCl}_{3}$ at $400 \mathrm{MH}$
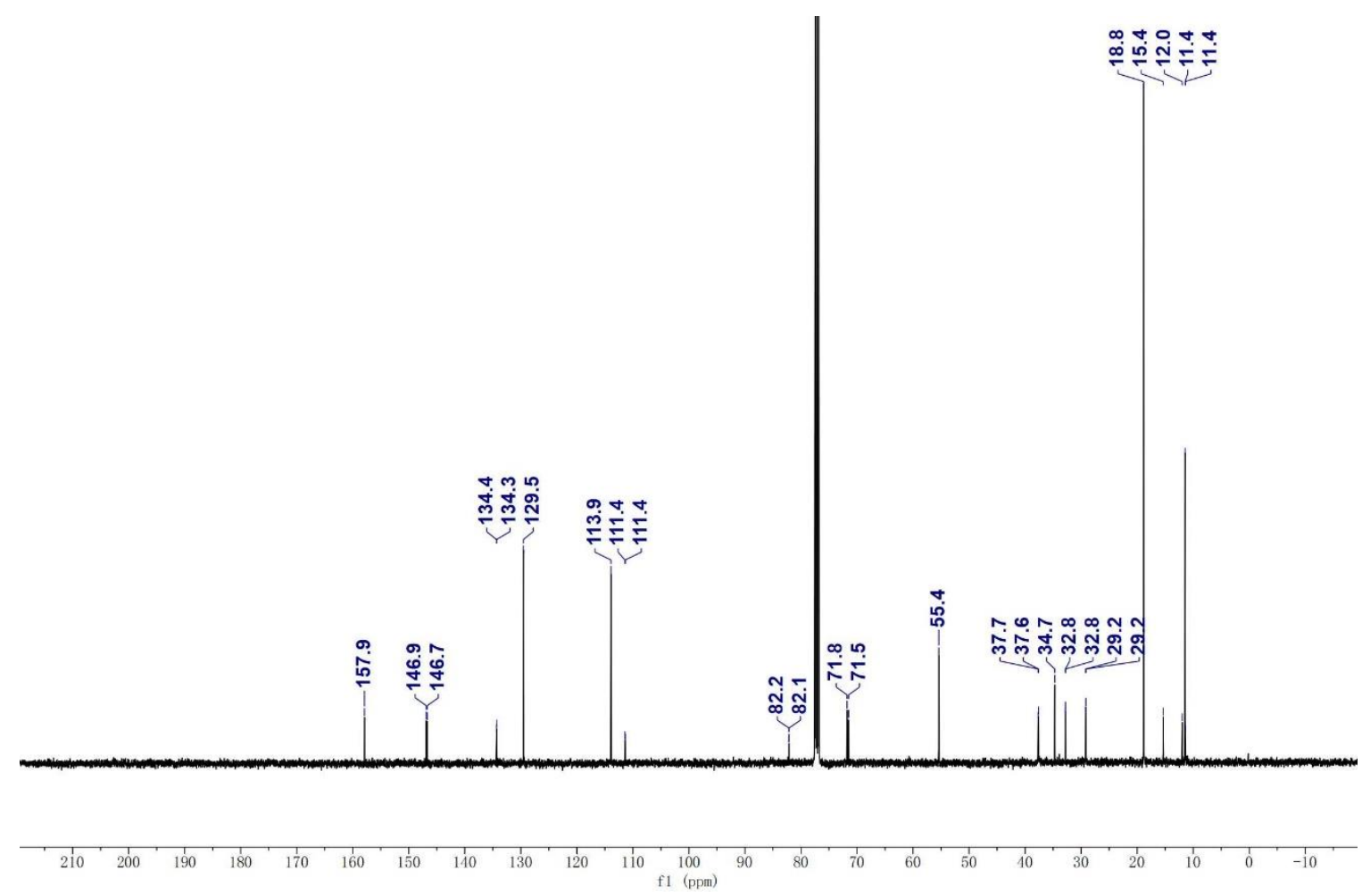

${ }^{13} \mathrm{C}$ NMR spectrum of $\mathbf{3 l}$ was measured in $\mathrm{CDCl}_{3}$ at $101 \mathrm{MHz}$ 

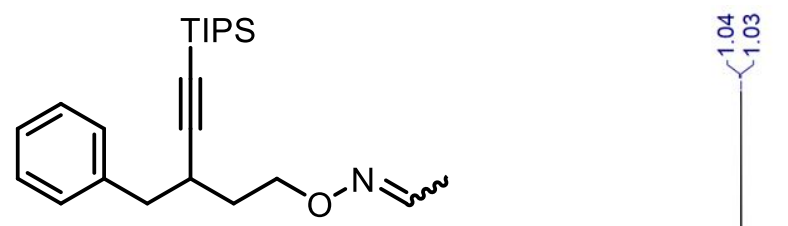

$3 m$

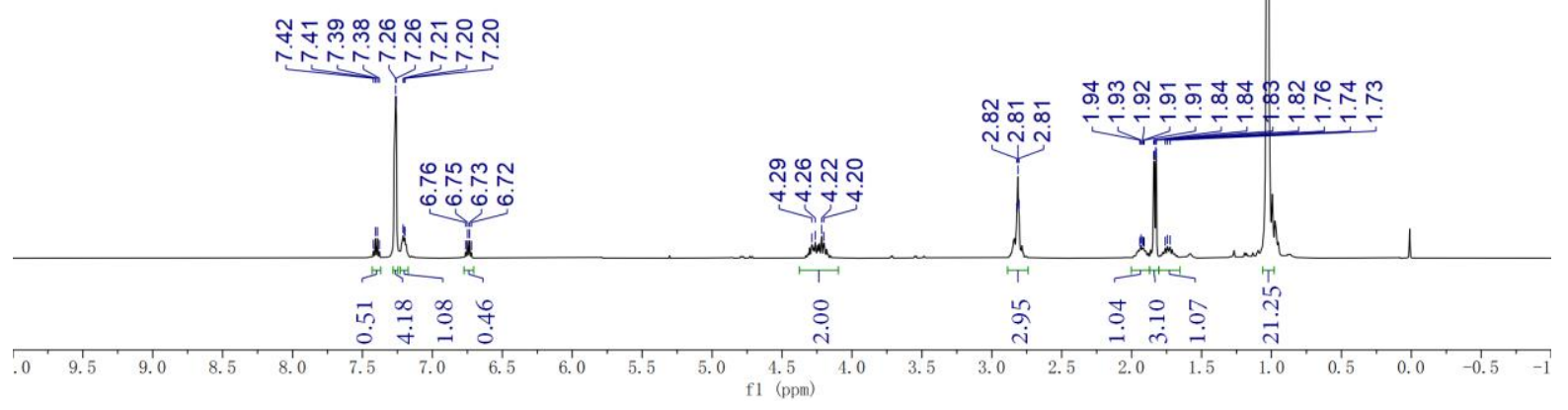

${ }^{1} \mathrm{H}$ NMR spectrum of $\mathbf{3 m}$ was measured in $\mathrm{CDCl}_{3}$ at $400 \mathrm{MH}$
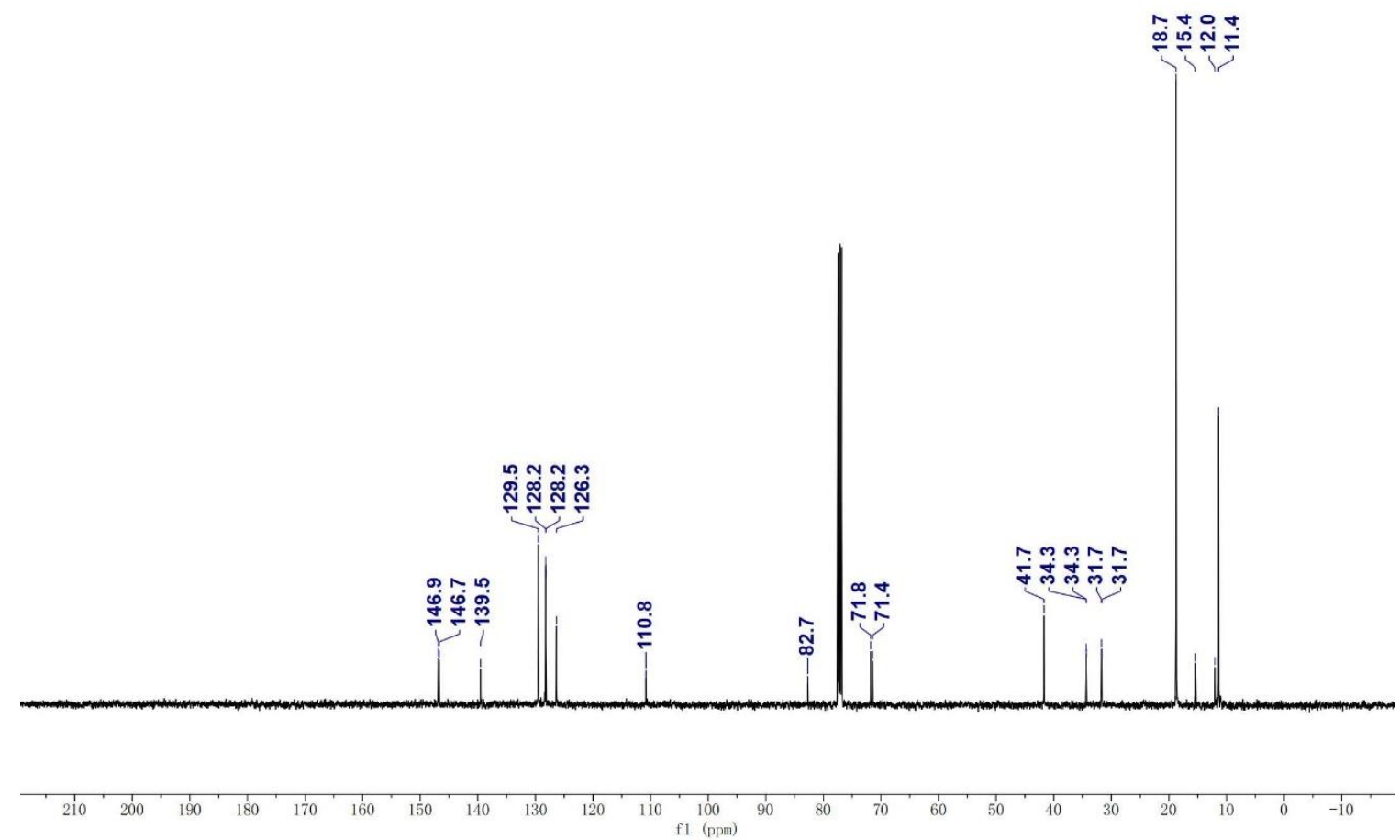

${ }^{13} \mathrm{C}$ NMR spectrum of $\mathbf{3 m}$ was measured in $\mathrm{CDCl}_{3}$ at $101 \mathrm{MHz}$ 


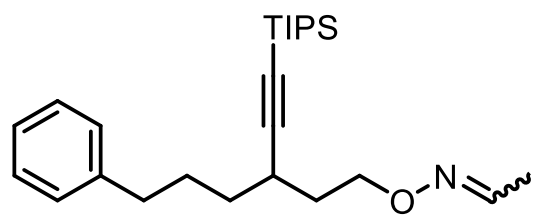

$3 n$

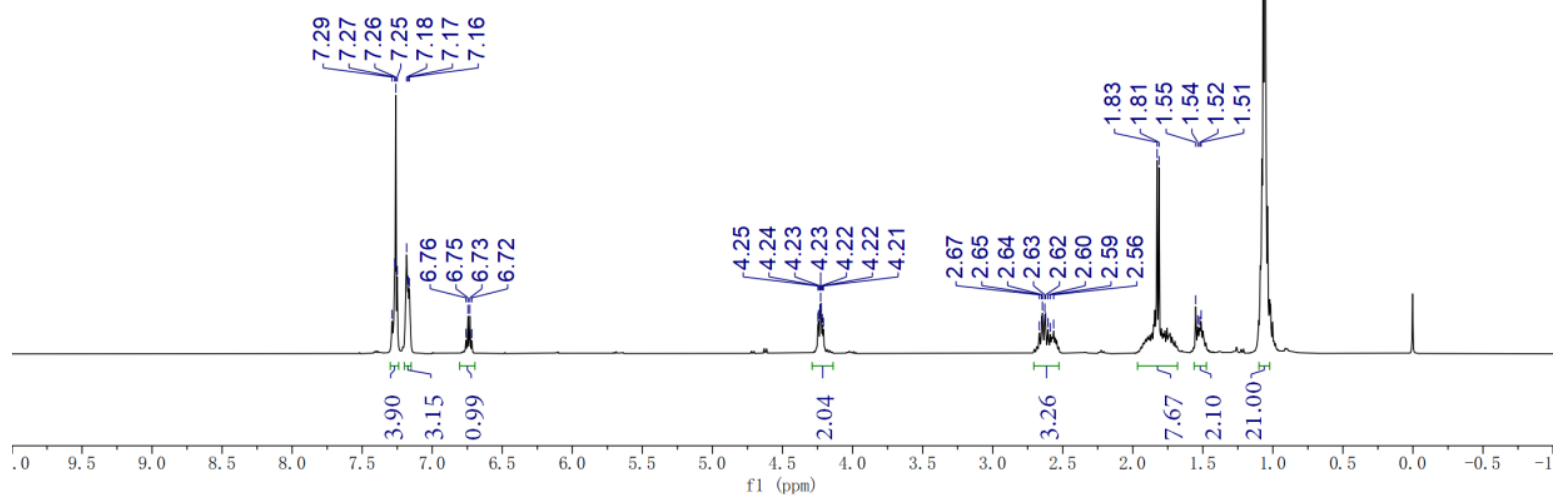

${ }^{1} \mathrm{H}$ NMR spectrum of $\mathbf{3 n}$ was measured in $\mathrm{CDCl}_{3}$ at $400 \mathrm{MH}$
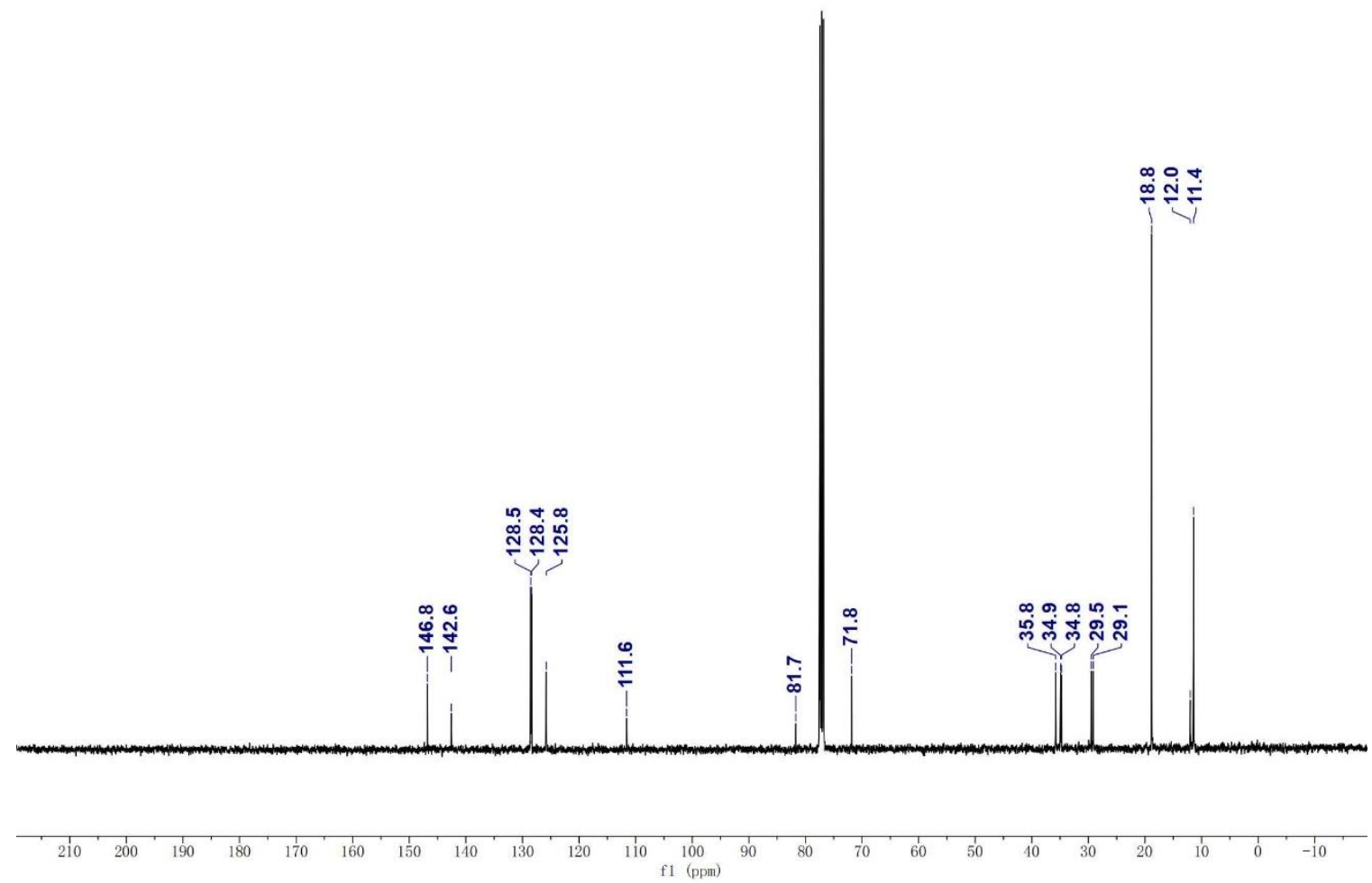

${ }^{13} \mathrm{C}$ NMR spectrum of $\mathbf{3 n}$ was measured in $\mathrm{CDCl}_{3}$ at $101 \mathrm{MHz}$ 


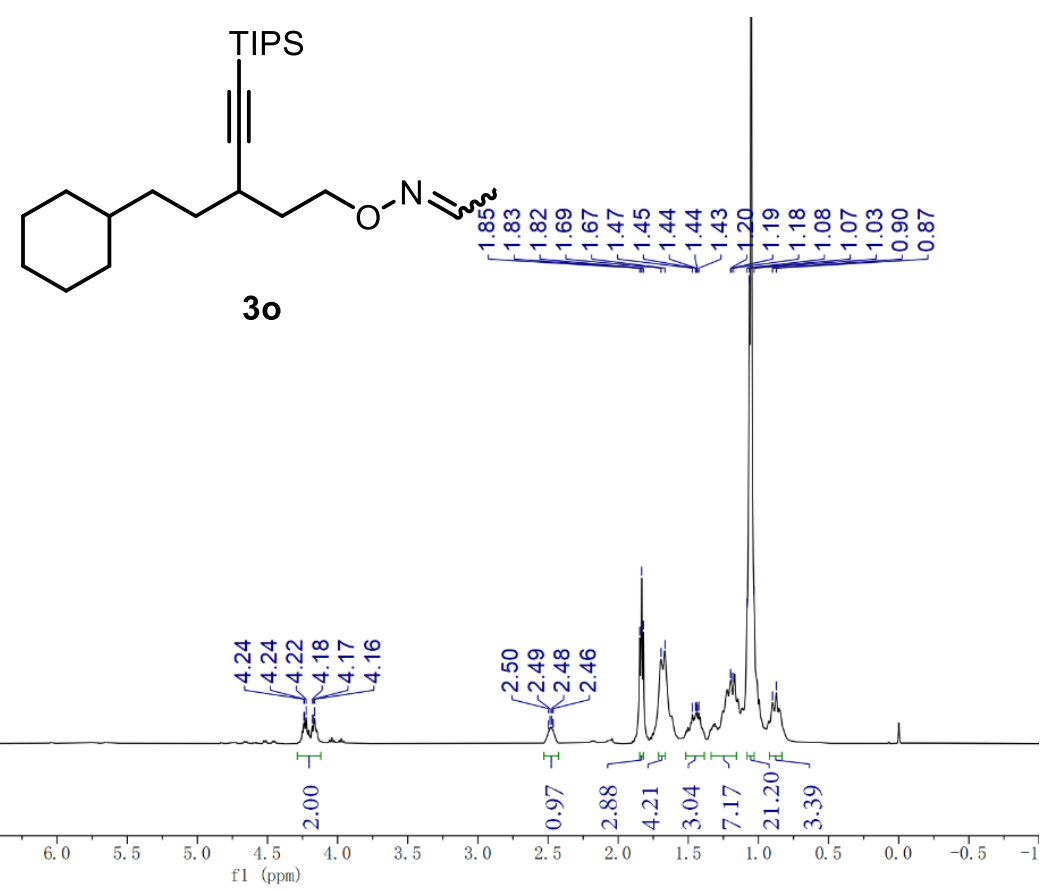

${ }^{1} \mathrm{H}$ NMR spectrum of 30 was measured in $\mathrm{CDCl}_{3}$ at $400 \mathrm{MH}$
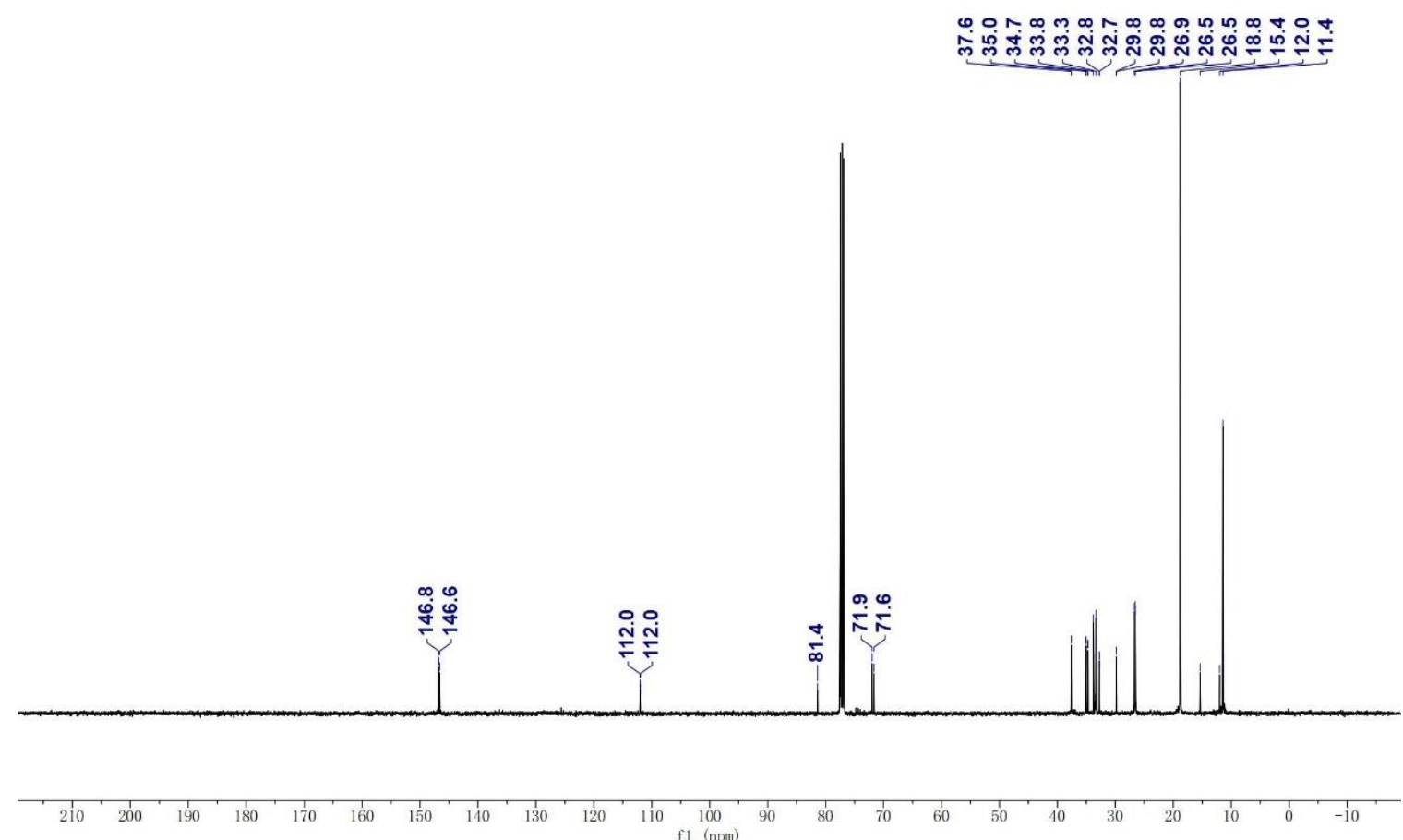

${ }^{13} \mathrm{C}$ NMR spectrum of 30 was measured in $\mathrm{CDCl}_{3}$ at $101 \mathrm{MHz}$ 

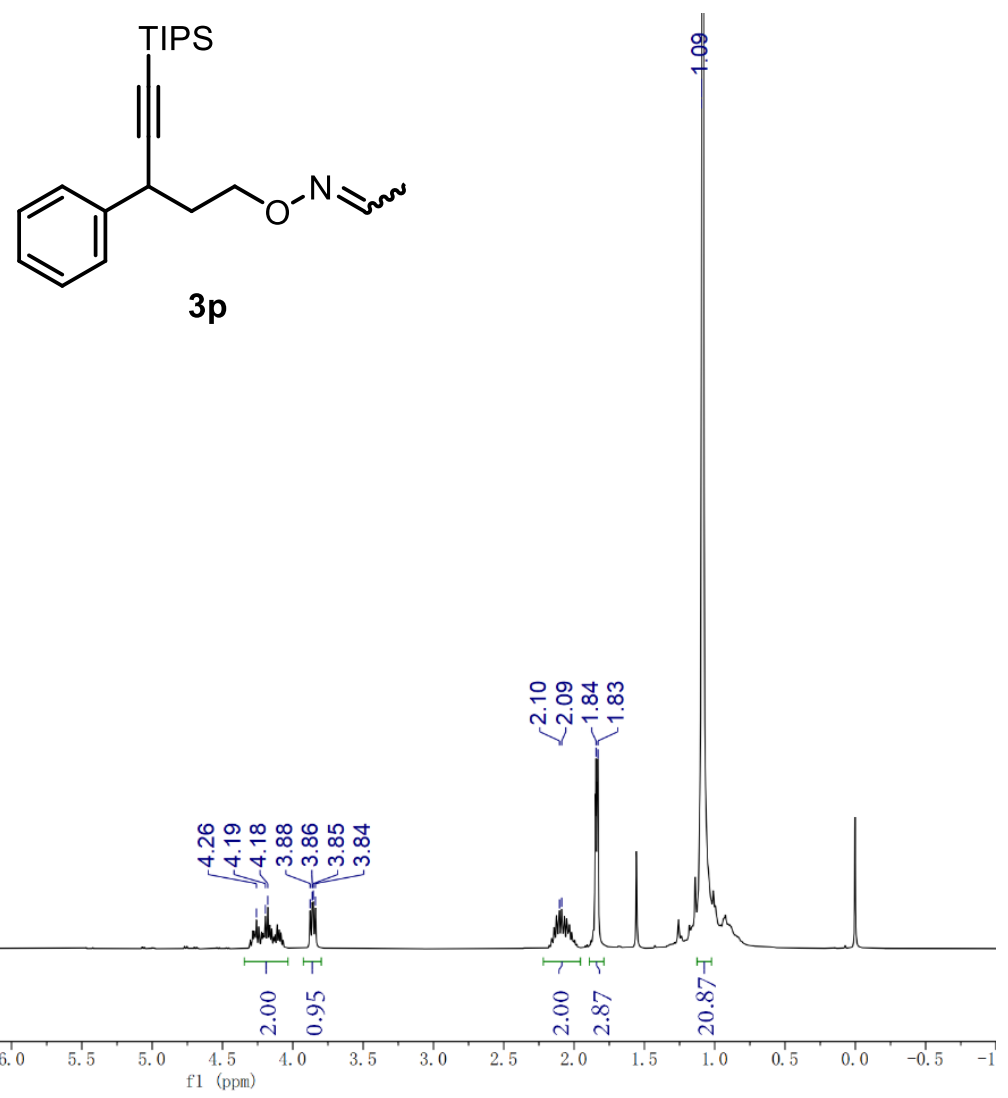

${ }^{1} \mathrm{H}$ NMR spectrum of $\mathbf{3 p}$ was measured in $\mathrm{CDCl}_{3}$ at $400 \mathrm{MH}$
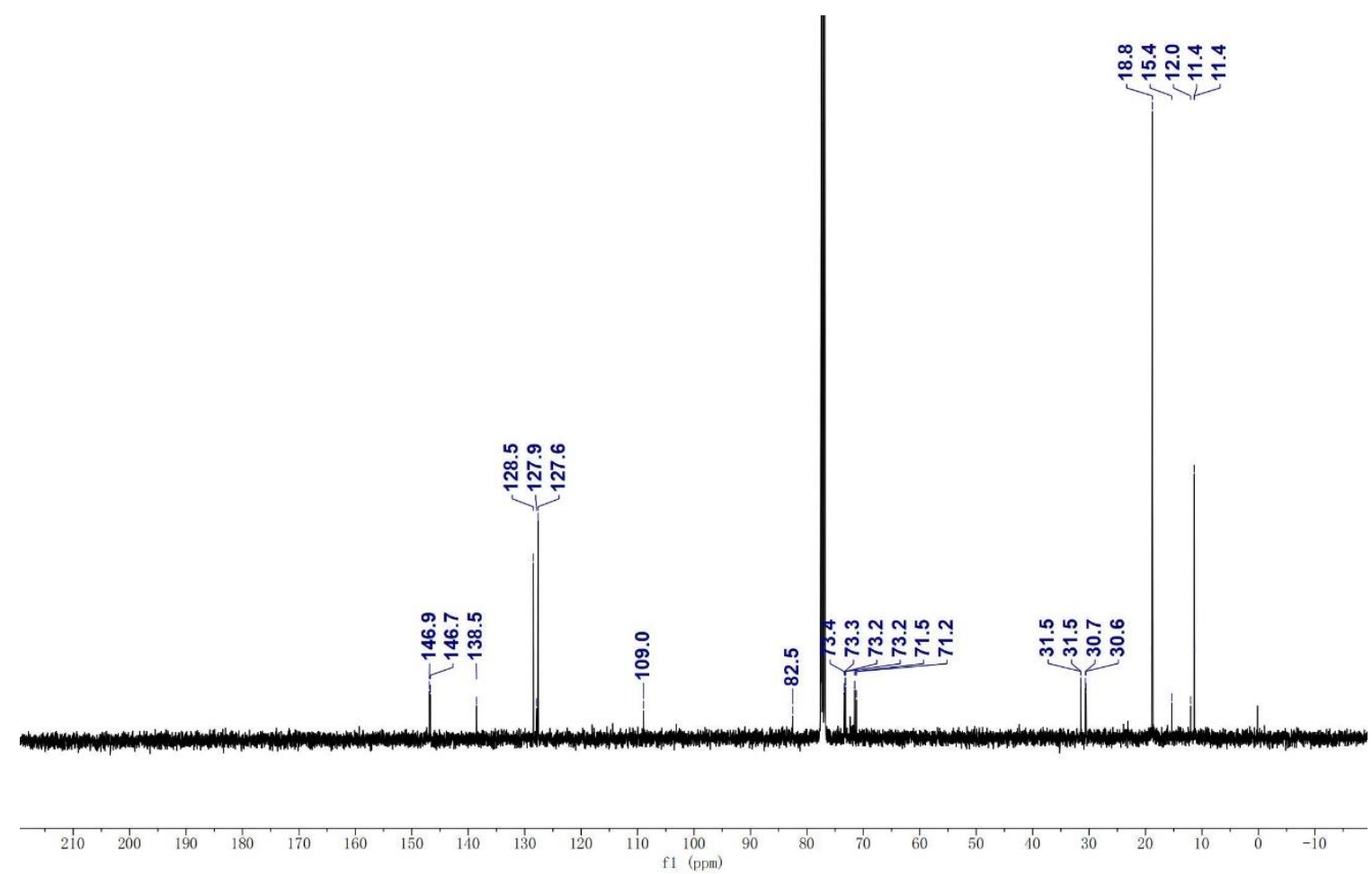

${ }^{13} \mathrm{C}$ NMR spectrum of $\mathbf{3 p}$ was measured in $\mathrm{CDCl}_{3}$ at $101 \mathrm{MHz}$ 
<smiles>CC=NOCCC(C#CC(F)F)C1CCCCC1</smiles>

$3 q$

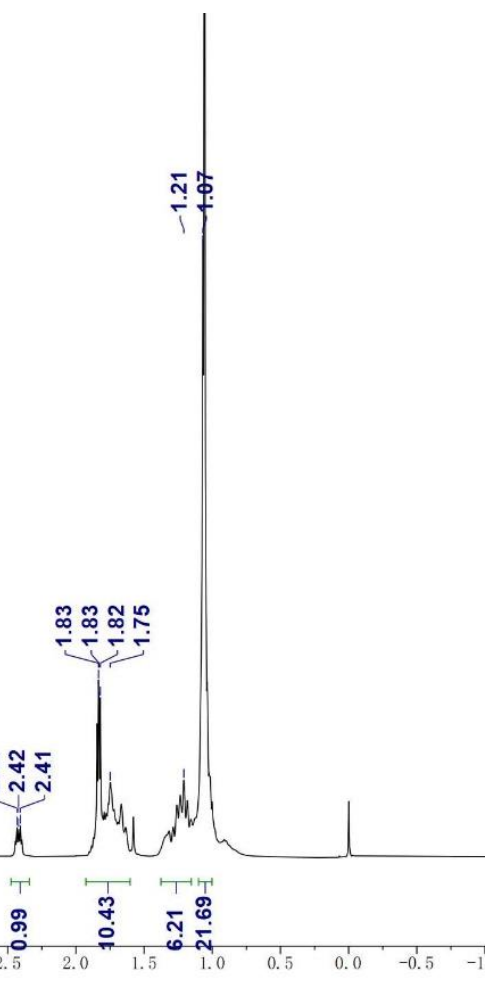

${ }^{1} \mathrm{H}$ NMR spectrum of $\mathbf{3 q}$ was measured in $\mathrm{CDCl}_{3}$ at $400 \mathrm{MH}$
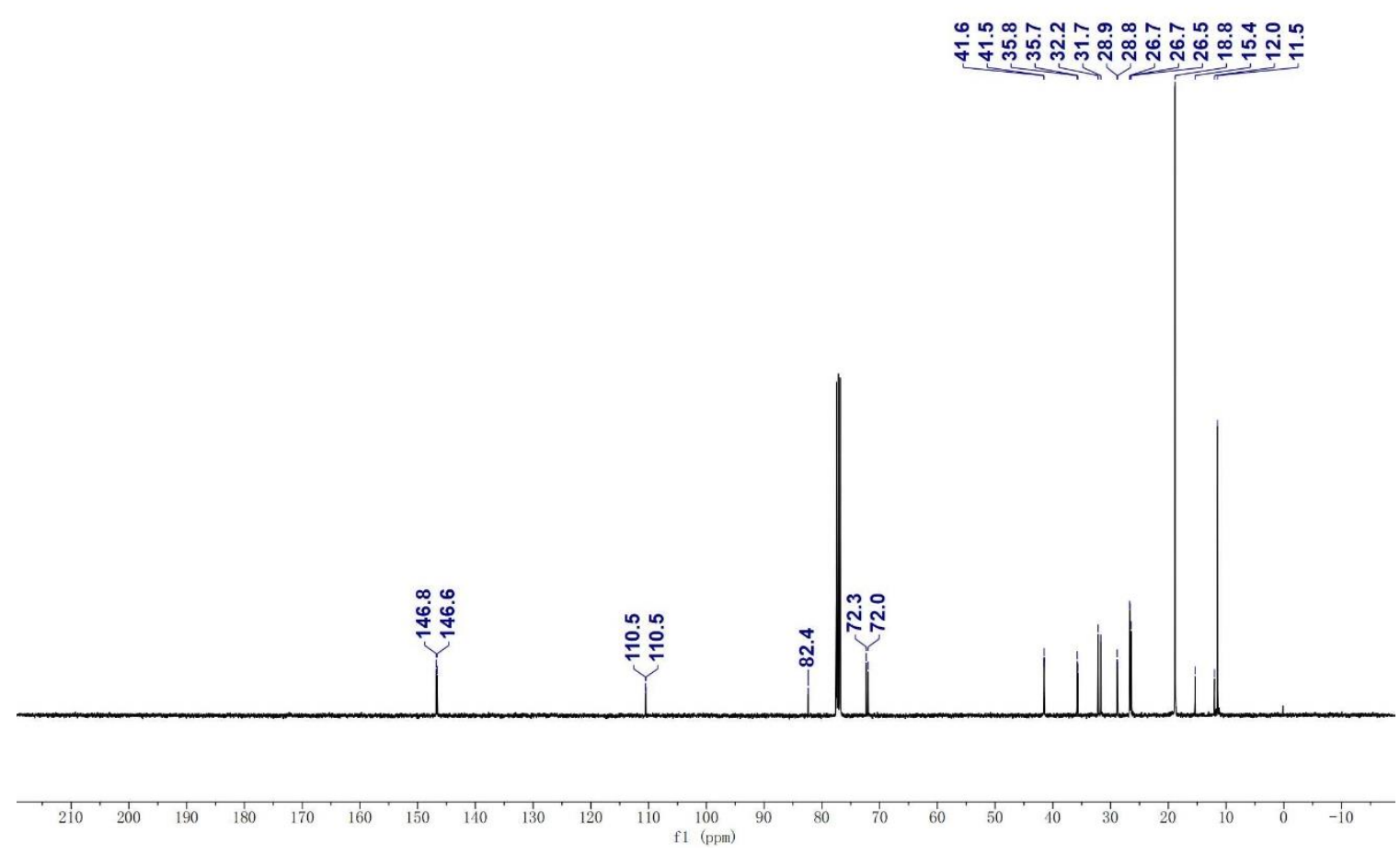

${ }^{13} \mathrm{C}$ NMR spectrum of $\mathbf{3 q}$ was measured in $\mathrm{CDCl}_{3}$ at $101 \mathrm{MHz}$ 

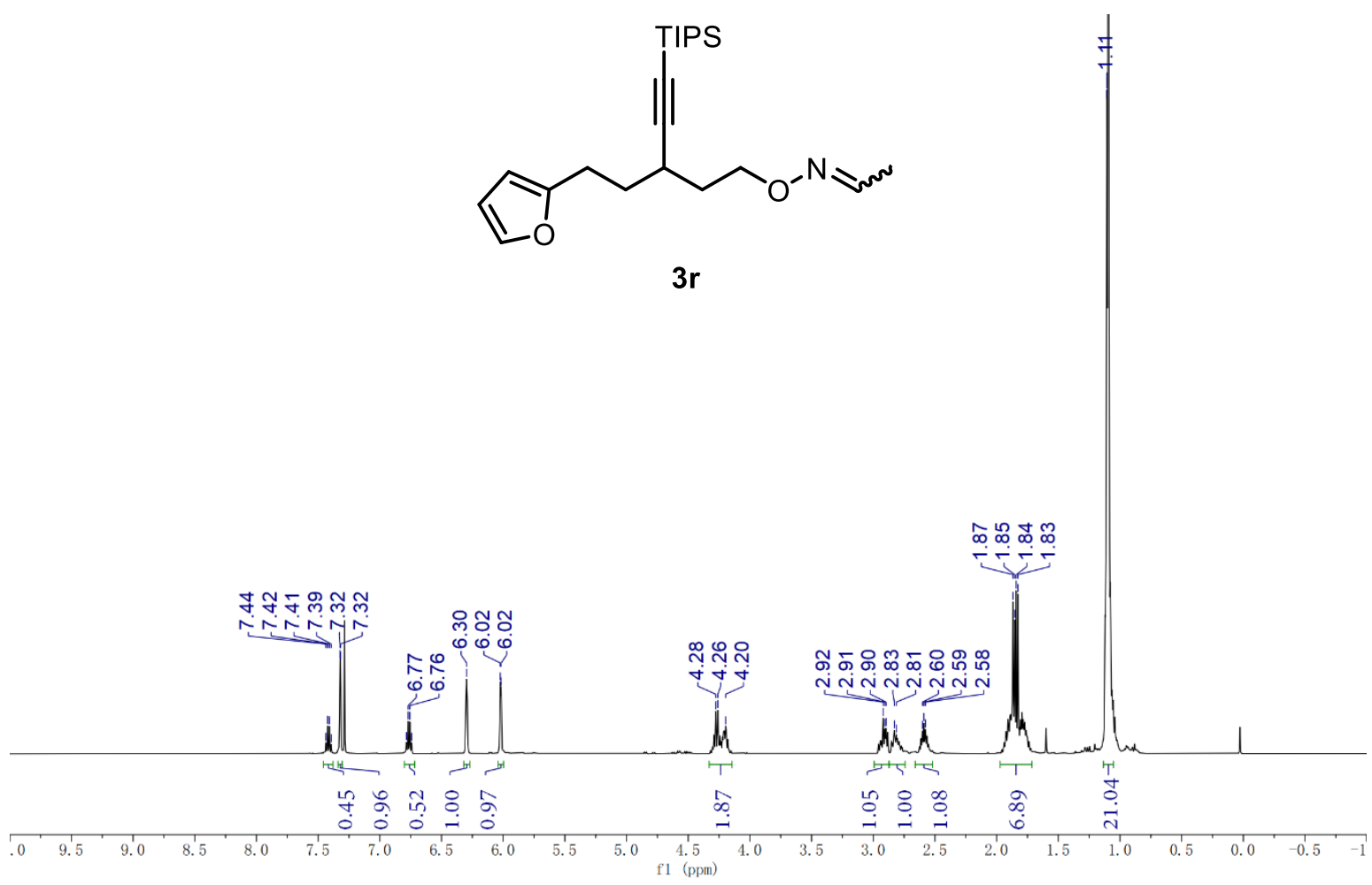

${ }^{1} \mathrm{H}$ NMR spectrum of 3r was measured in $\mathrm{CDCl}_{3}$ at $400 \mathrm{MH}$

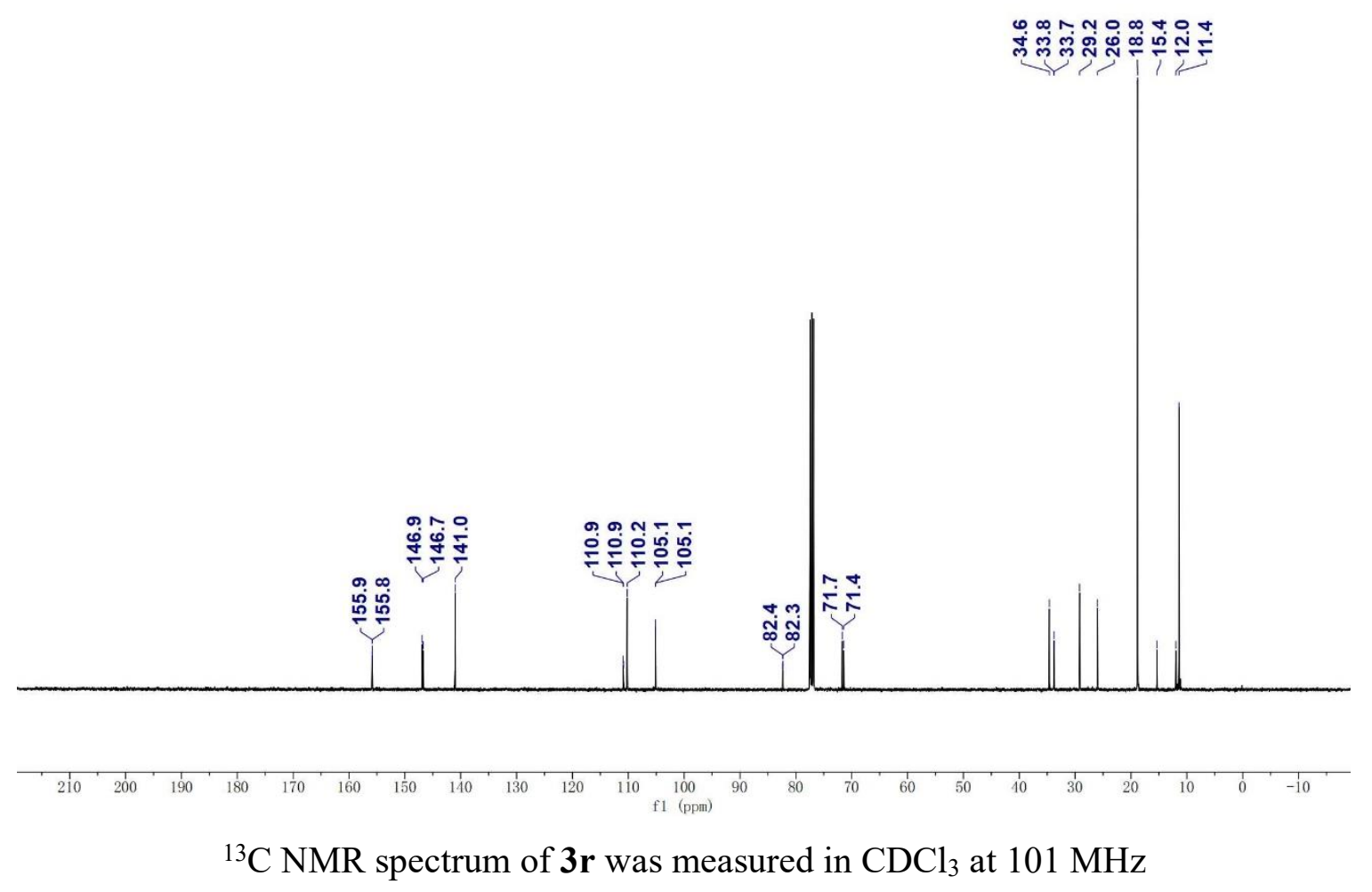




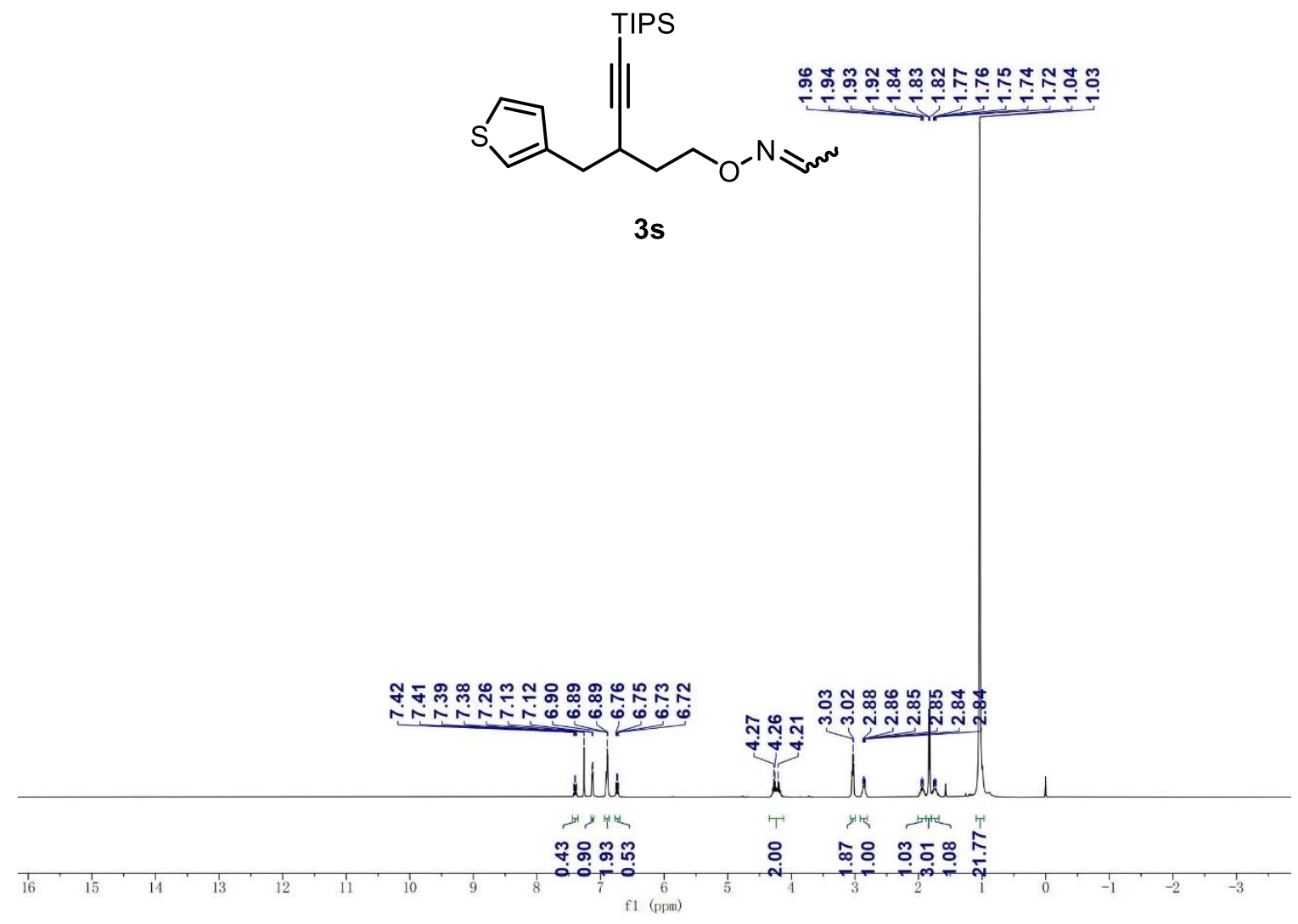

${ }^{1} \mathrm{H}$ NMR spectrum of $3 \mathbf{s}$ was measured in $\mathrm{CDCl}_{3}$ at $400 \mathrm{MH}$
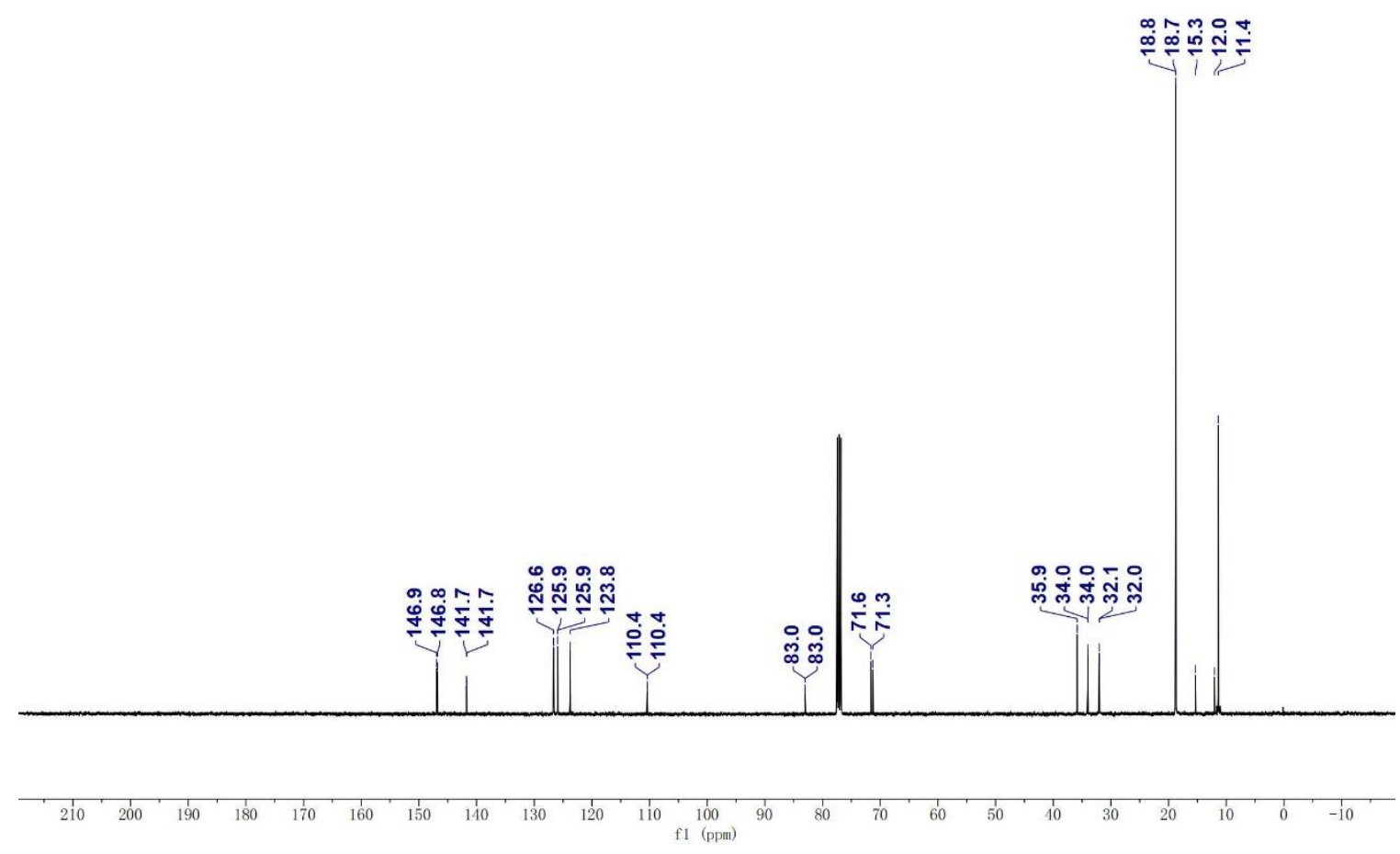

${ }^{13} \mathrm{C}$ NMR spectrum of $3 \mathrm{~s}$ was measured in $\mathrm{CDCl}_{3}$ at $101 \mathrm{MHz}$ 

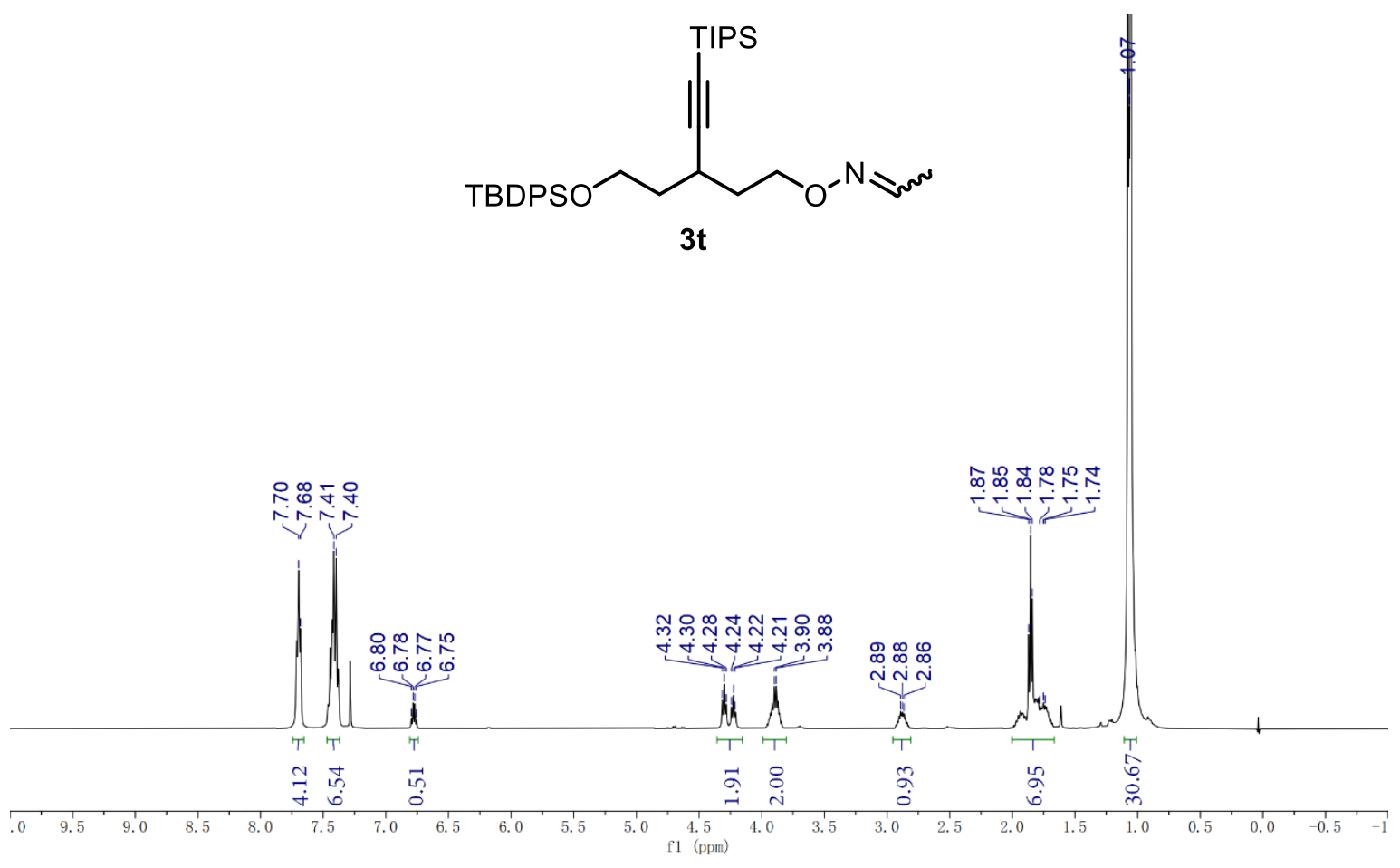

${ }^{1} \mathrm{H}$ NMR spectrum of $\mathbf{3 t}$ was measured in $\mathrm{CDCl}_{3}$ at $400 \mathrm{MH}$
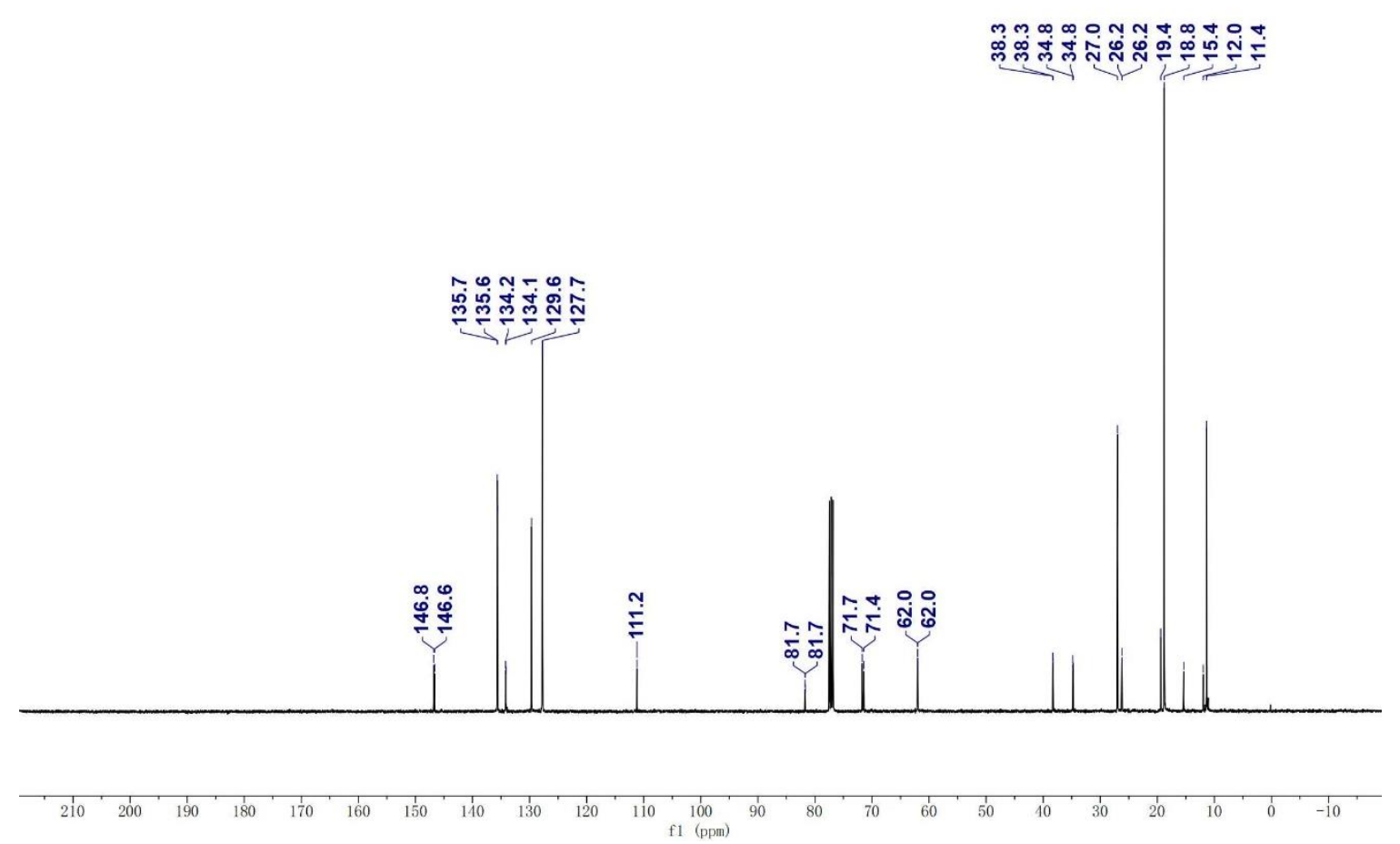

${ }^{13} \mathrm{C}$ NMR spectrum of $3 \mathrm{t}$ was measured in $\mathrm{CDCl}_{3}$ at $101 \mathrm{MHz}$ 

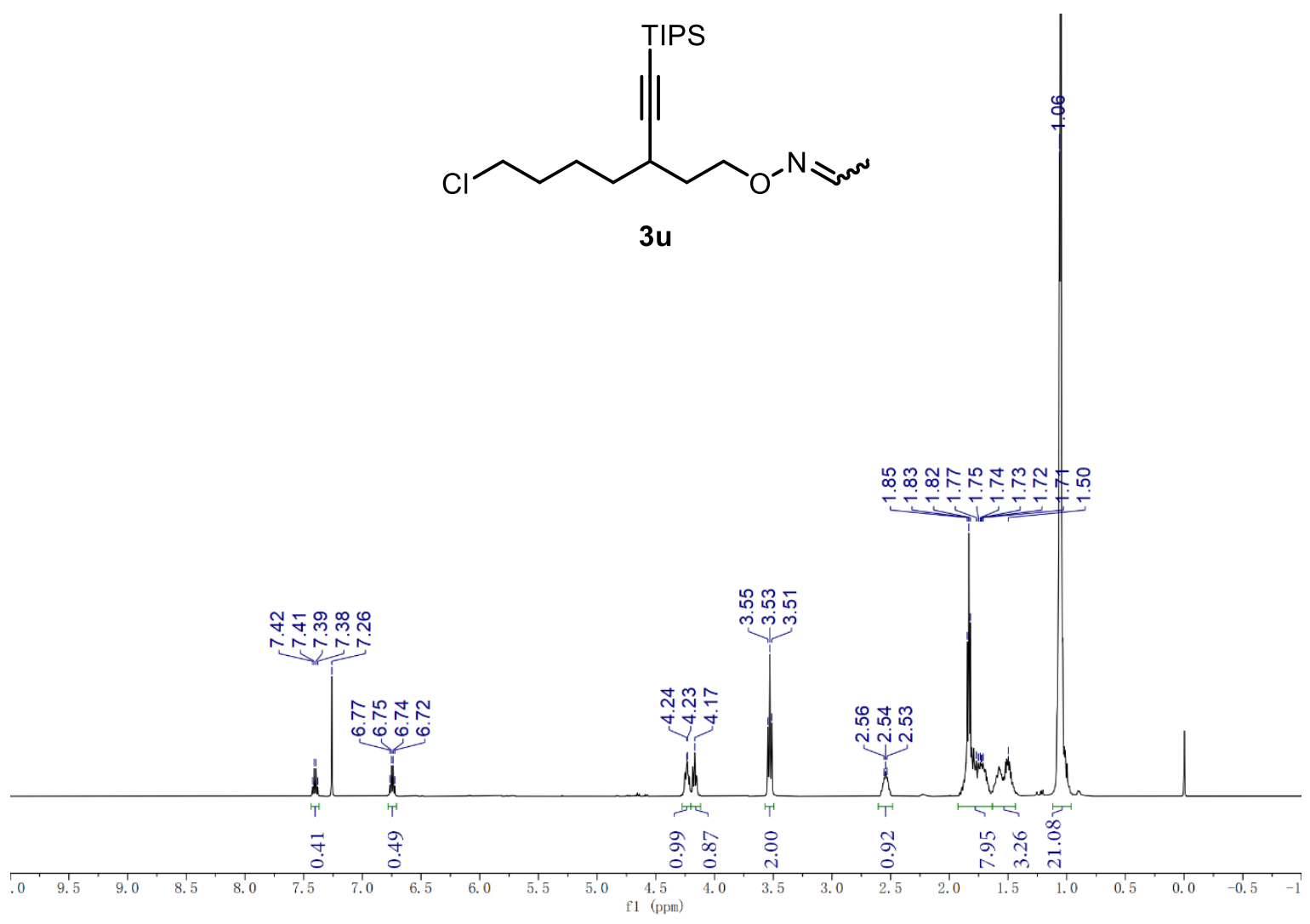

${ }^{1} \mathrm{H}$ NMR spectrum of $3 \mathbf{u}$ was measured in $\mathrm{CDCl}_{3}$ at $400 \mathrm{MH}$
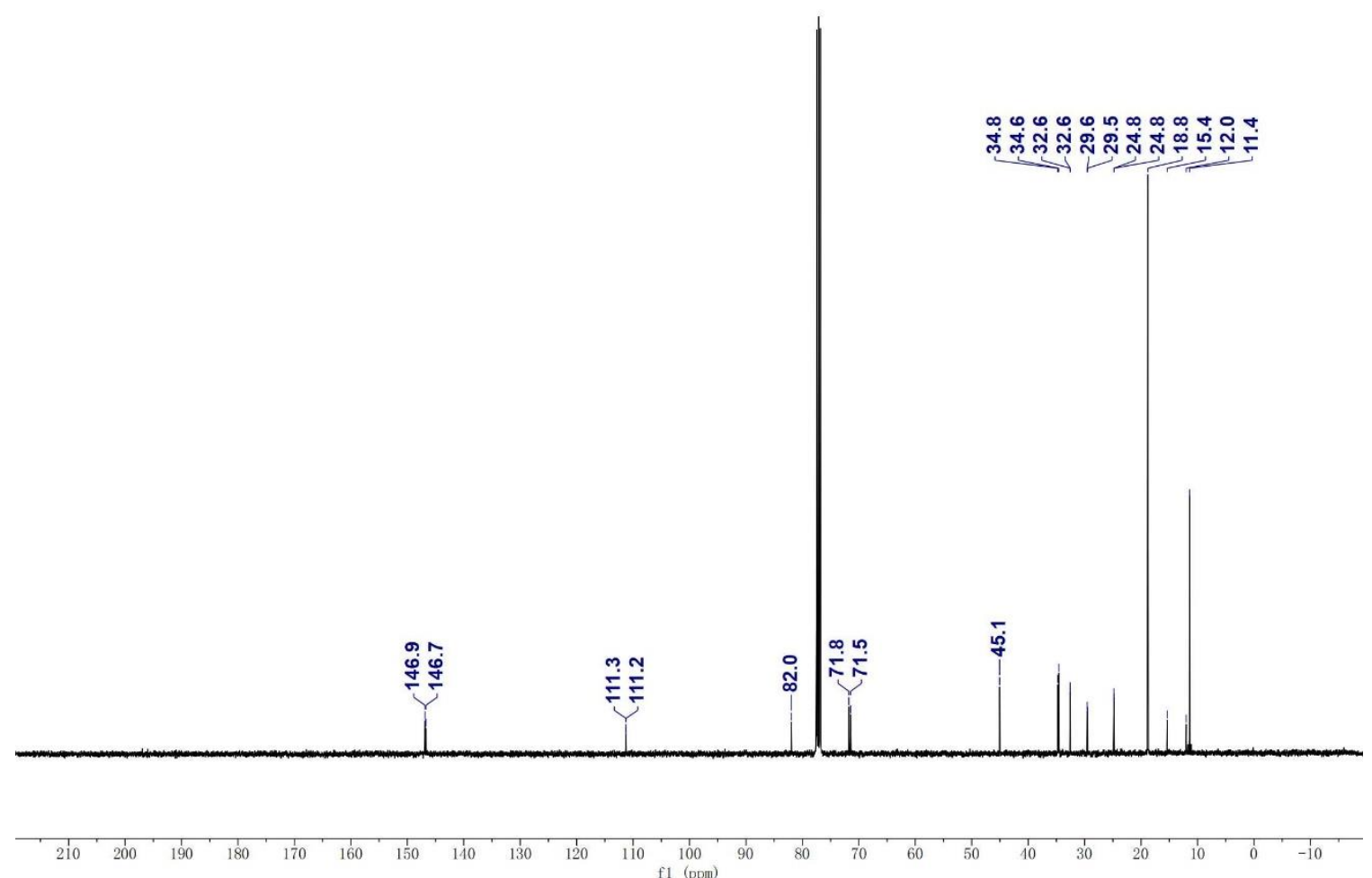

${ }^{13} \mathrm{C}$ NMR spectrum of $\mathbf{3 u}$ was measured in $\mathrm{CDCl}_{3}$ at $101 \mathrm{MHz}$ 

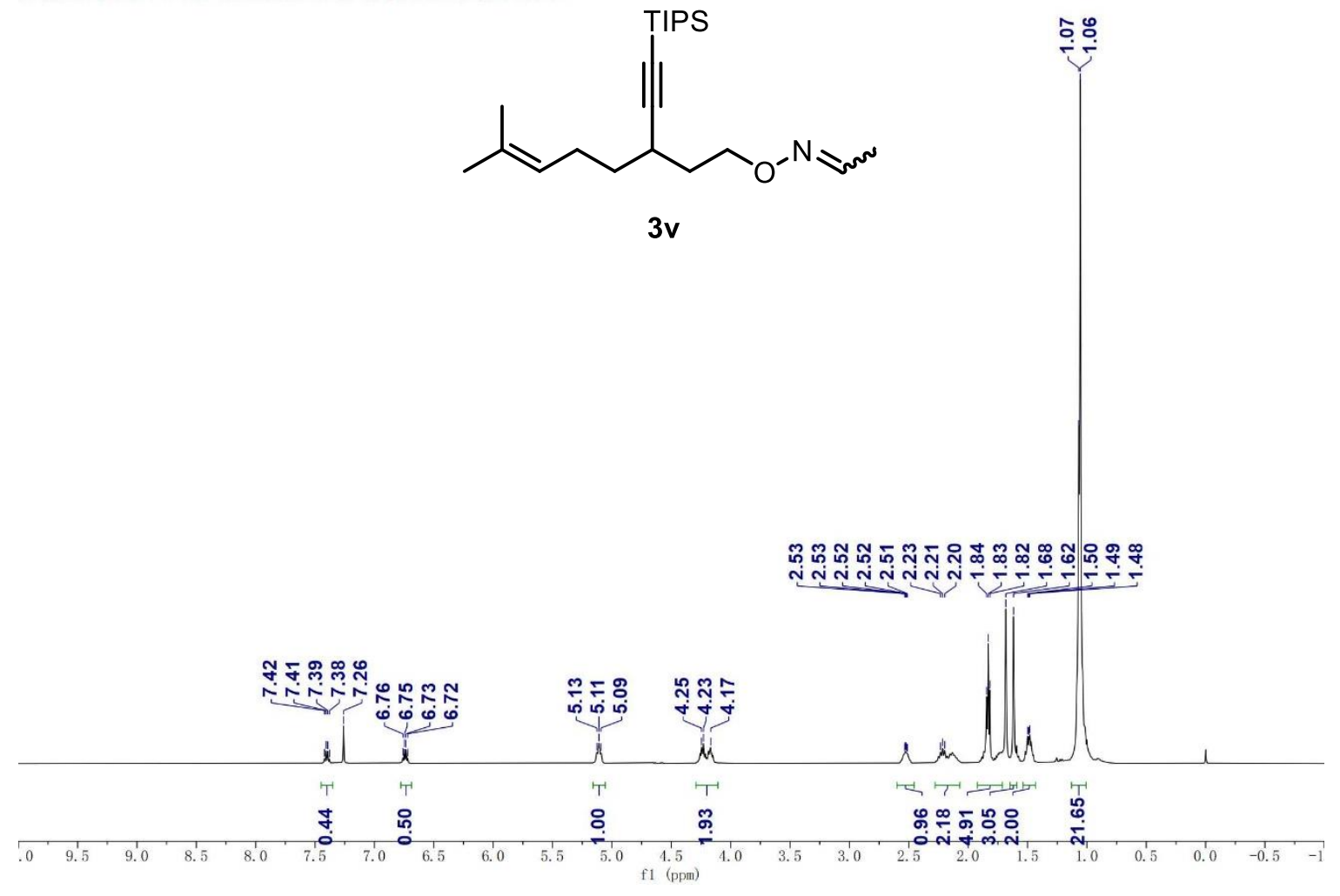

${ }^{1} \mathrm{H}$ NMR spectrum of $\mathbf{3 v}$ was measured in $\mathrm{CDCl}_{3}$ at $400 \mathrm{MH}$
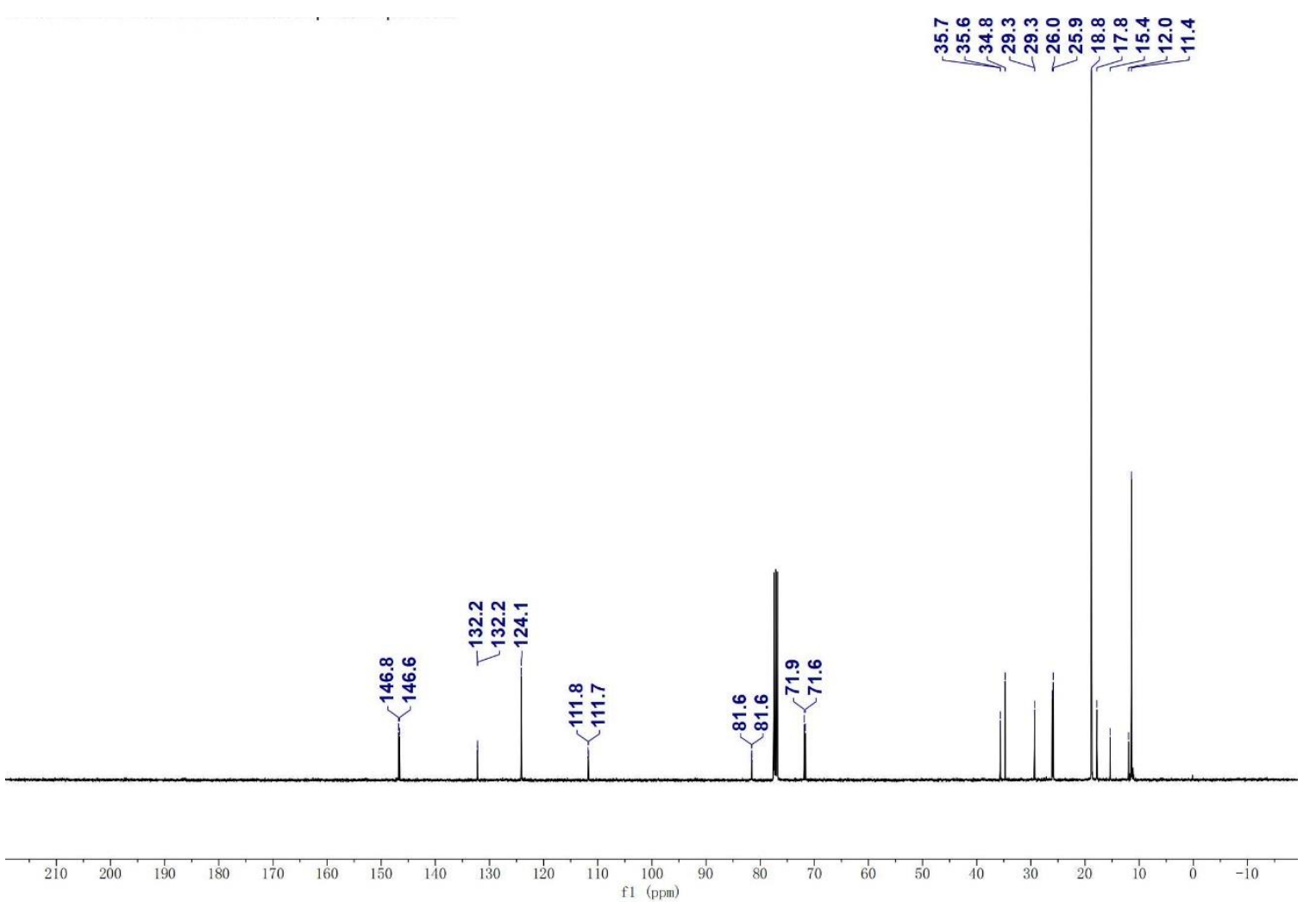

${ }^{13} \mathrm{C}$ NMR spectrum of $\mathbf{3 v}$ was measured in $\mathrm{CDCl}_{3}$ at $101 \mathrm{MHz}$ 
<smiles>CC=NOCCC(C#C[18OH])COCc1ccccc1</smiles>

$3 w$

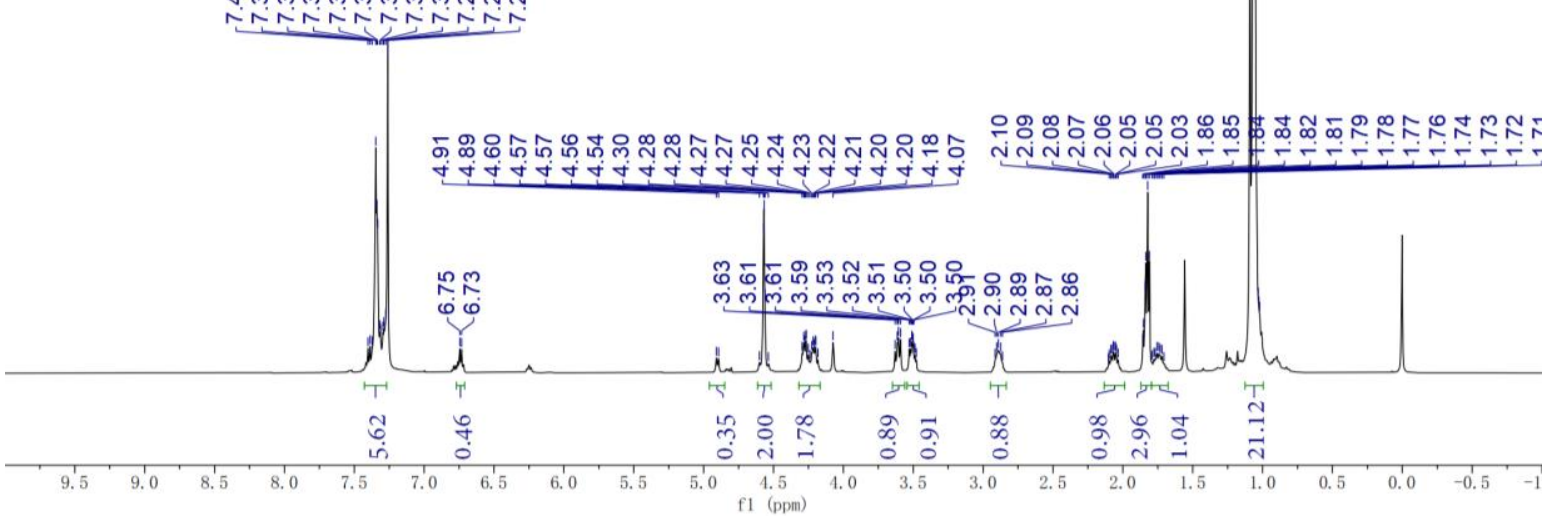

${ }^{1} \mathrm{H}$ NMR spectrum of $\mathbf{3 w}$ was measured in $\mathrm{CDCl}_{3}$ at $400 \mathrm{MH}$
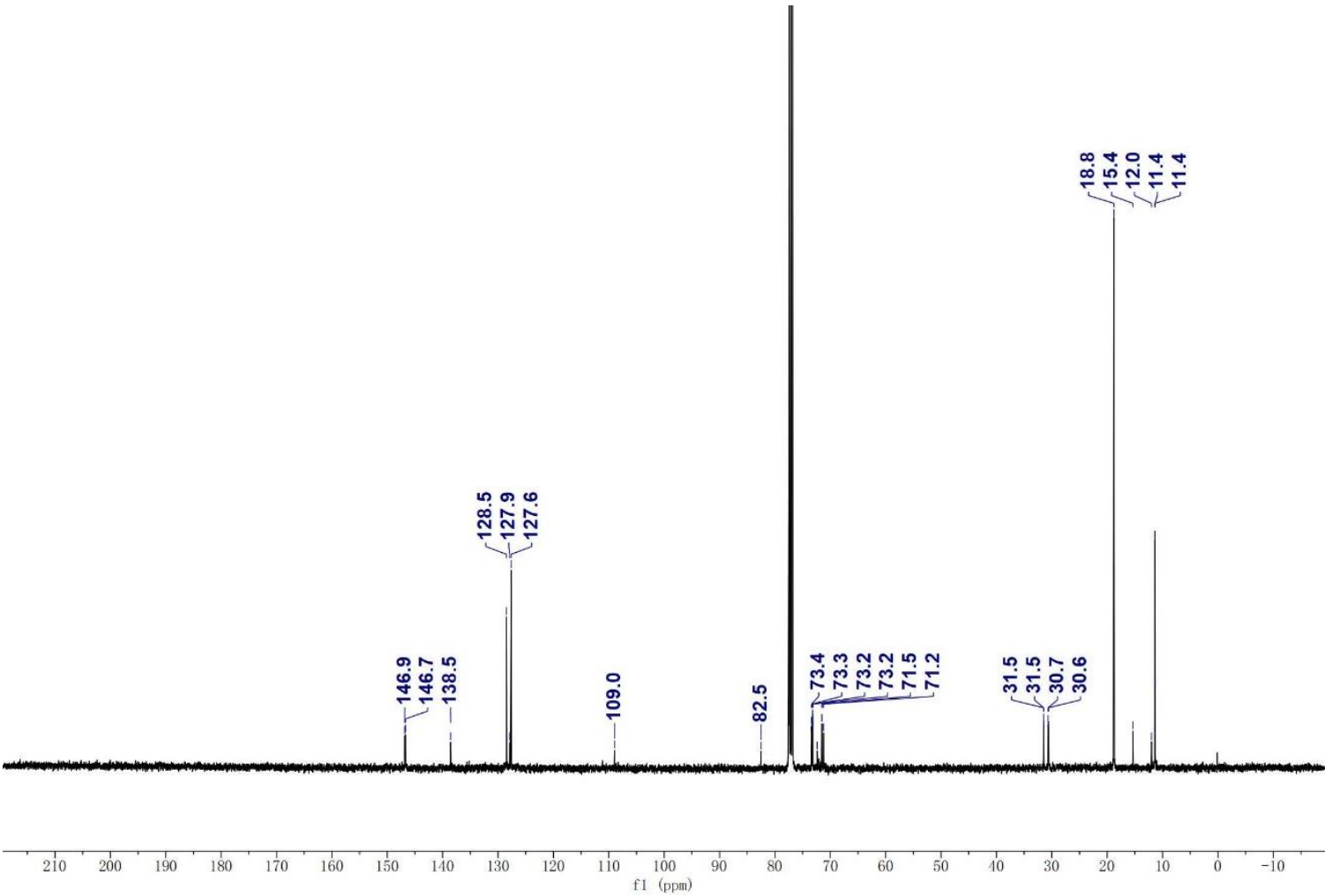

${ }^{13} \mathrm{C}$ NMR spectrum of $\mathbf{3 w}$ was measured in $\mathrm{CDCl}_{3}$ at $101 \mathrm{MHz}$ 


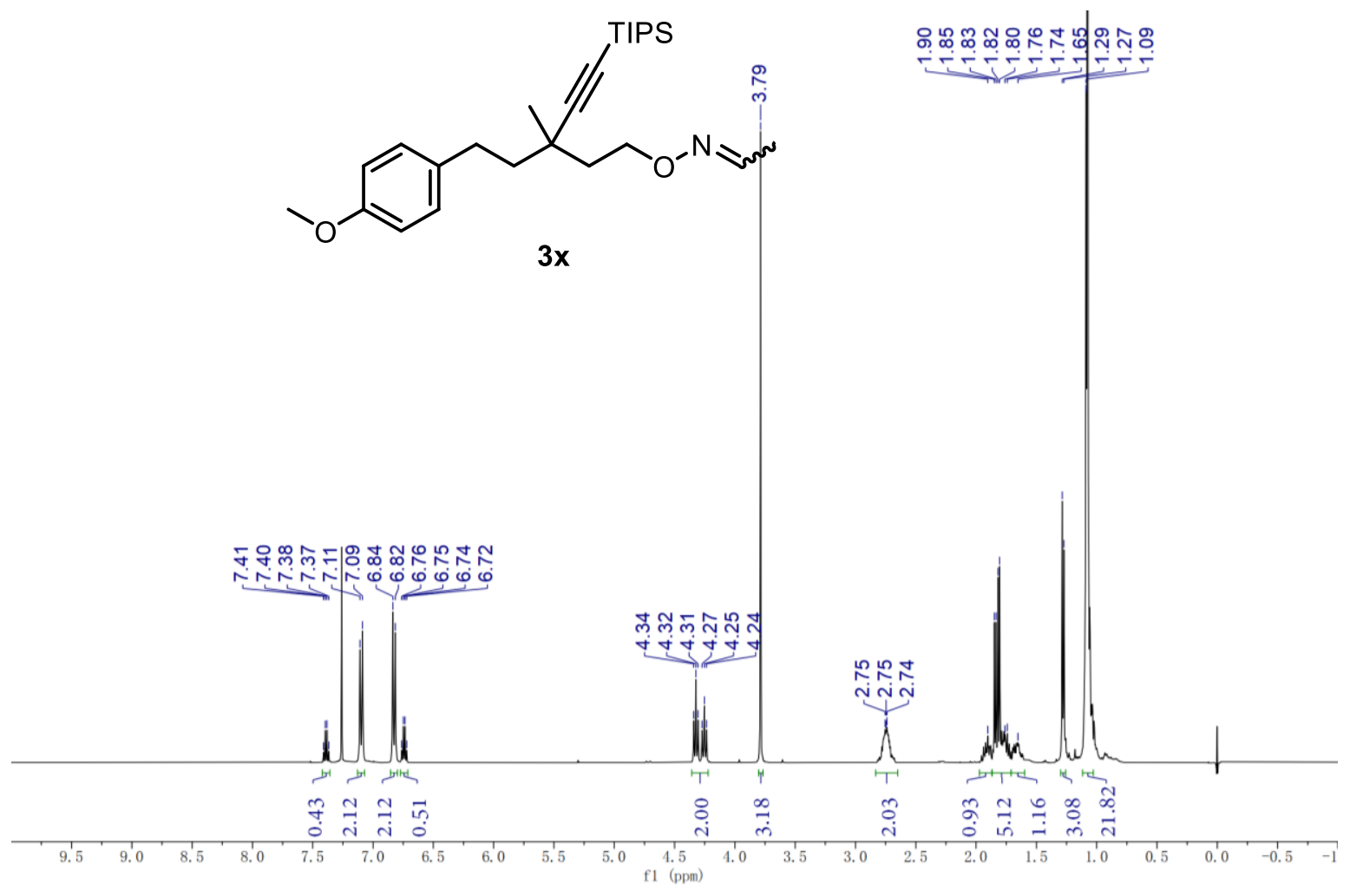

${ }^{1} \mathrm{H}$ NMR spectrum of $\mathbf{3 x}$ was measured in $\mathrm{CDCl}_{3}$ at $400 \mathrm{MH}$
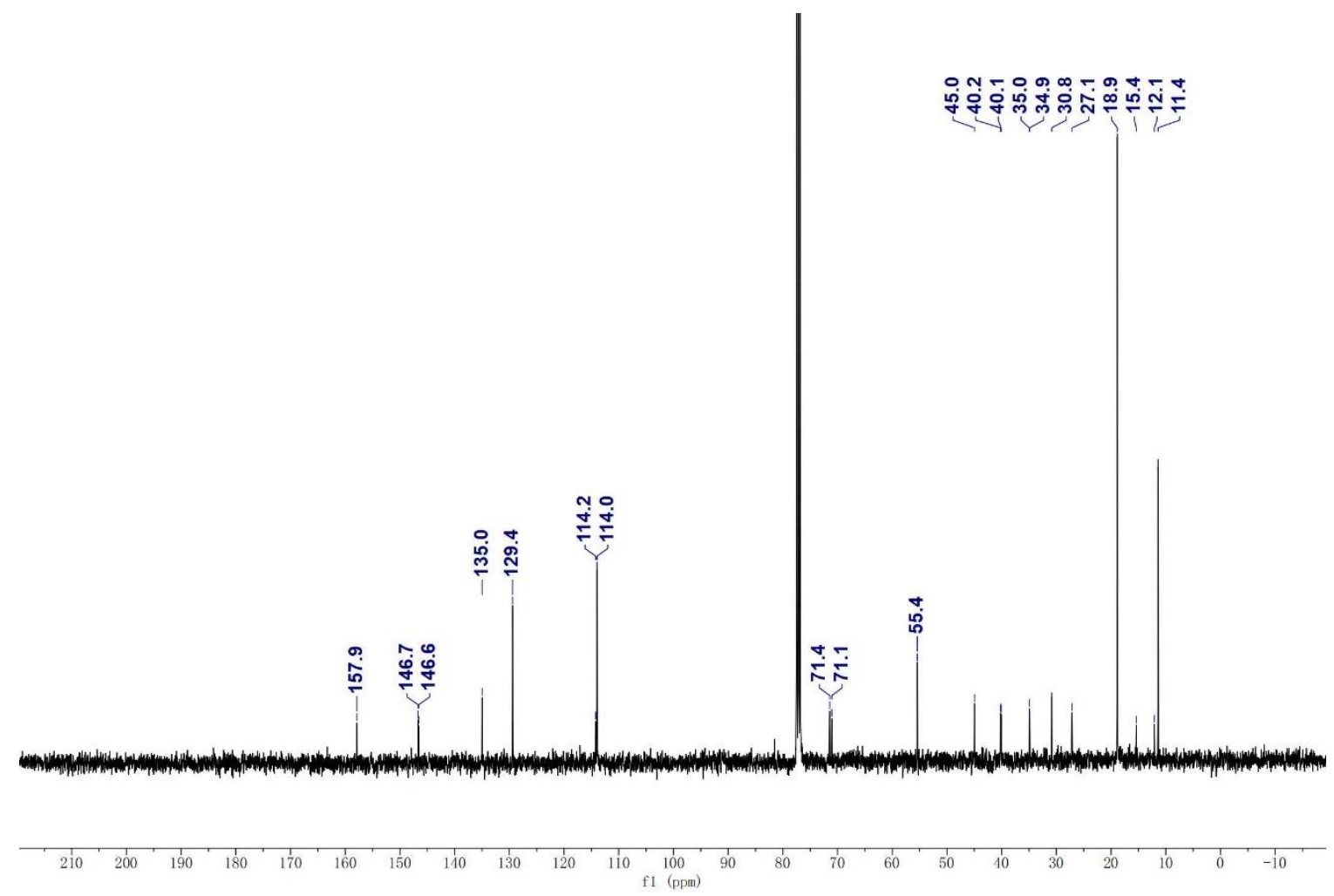

${ }^{13} \mathrm{C}$ NMR spectrum of $\mathbf{3 x}$ was measured in $\mathrm{CDCl}_{3}$ at $101 \mathrm{MHz}$ 


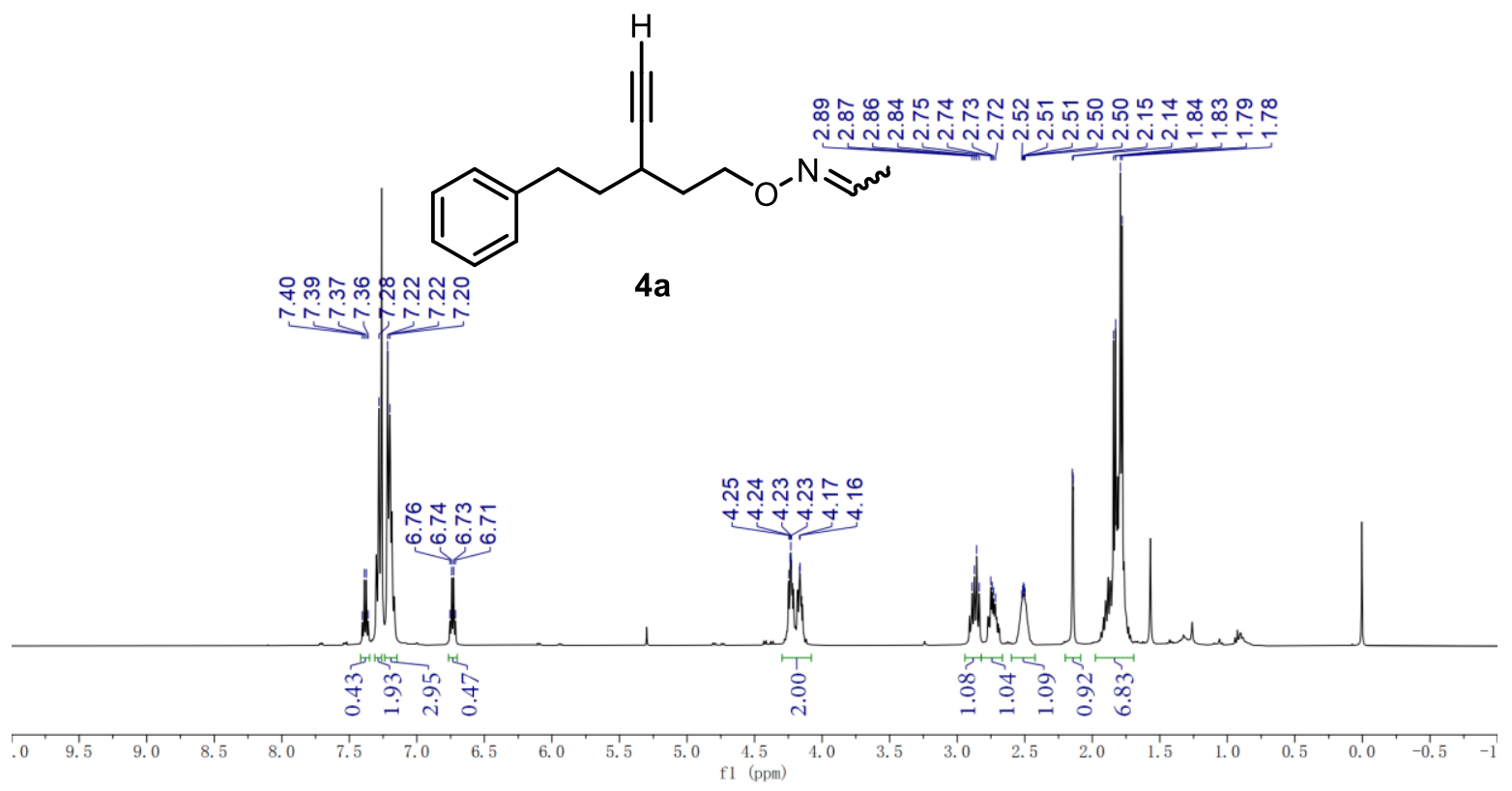

${ }^{1} \mathrm{H}$ NMR spectrum of $\mathbf{4 a}$ was measured in $\mathrm{CDCl}_{3}$ at $400 \mathrm{MH}$
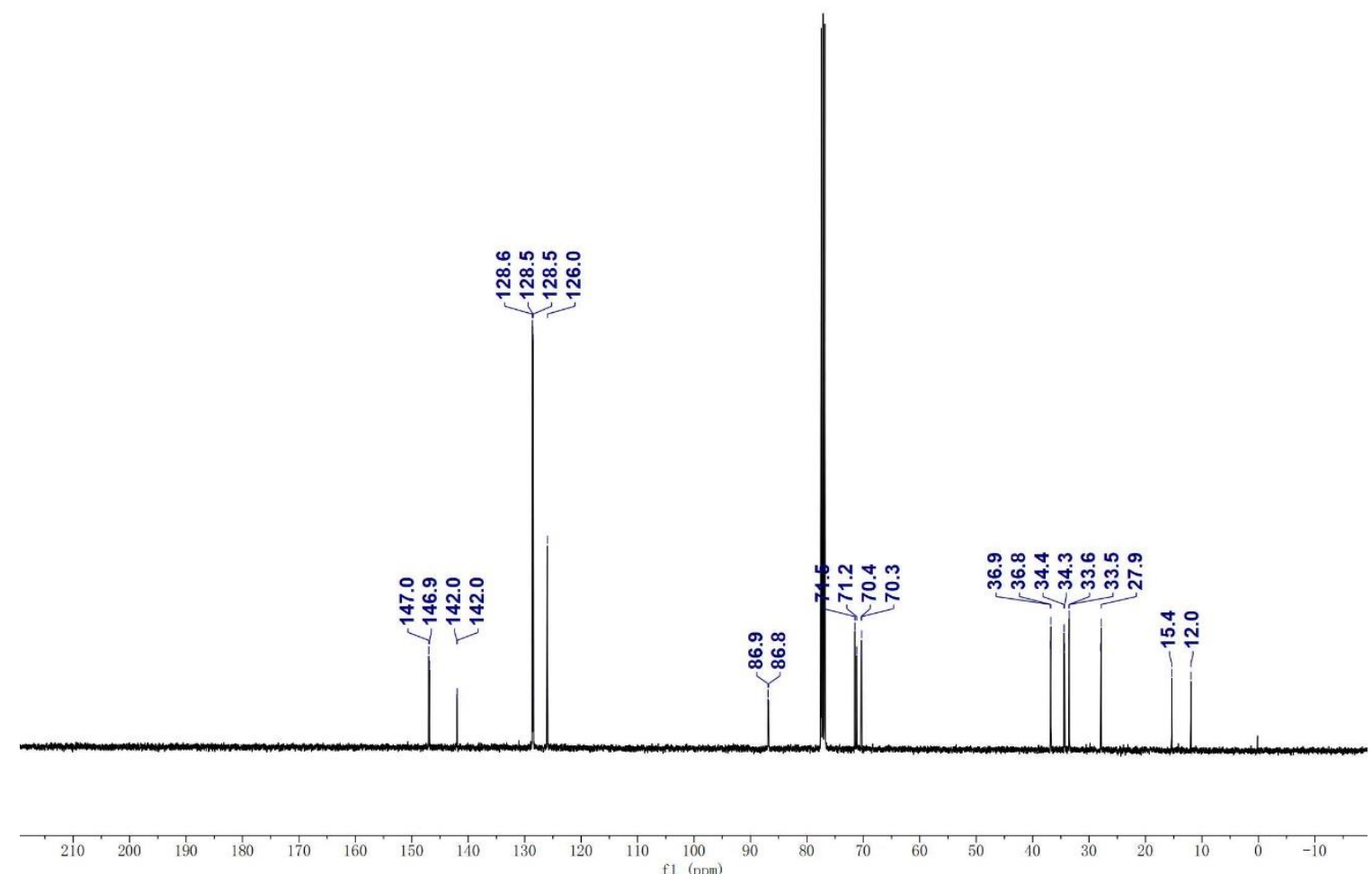

${ }^{13} \mathrm{C}$ NMR spectrum of $\mathbf{4 a}$ was measured in $\mathrm{CDCl}_{3}$ at $101 \mathrm{MHz}$ 


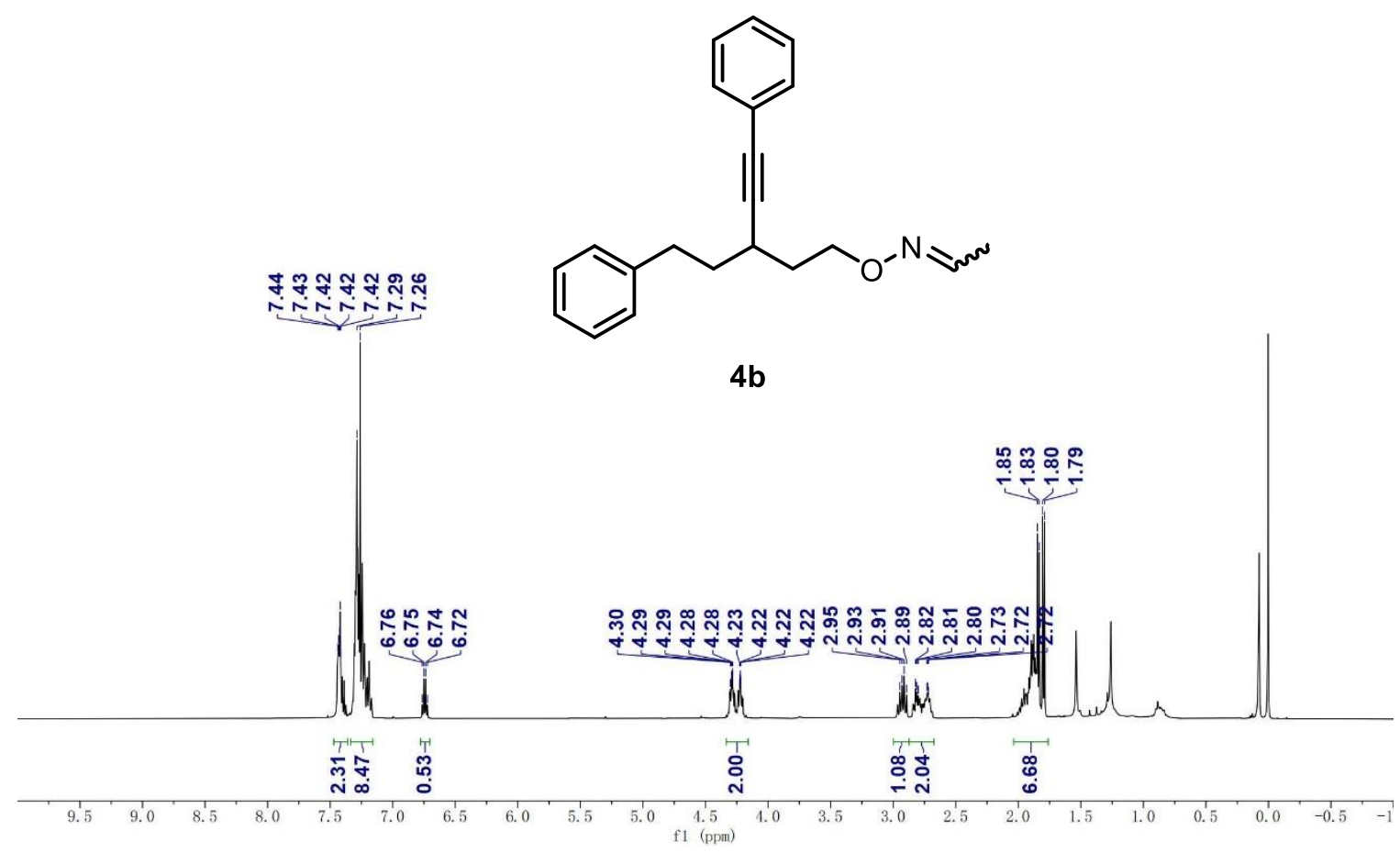

${ }^{1} \mathrm{H}$ NMR spectrum of $\mathbf{4 b}$ was measured in $\mathrm{CDCl}_{3}$ at $400 \mathrm{MH}$
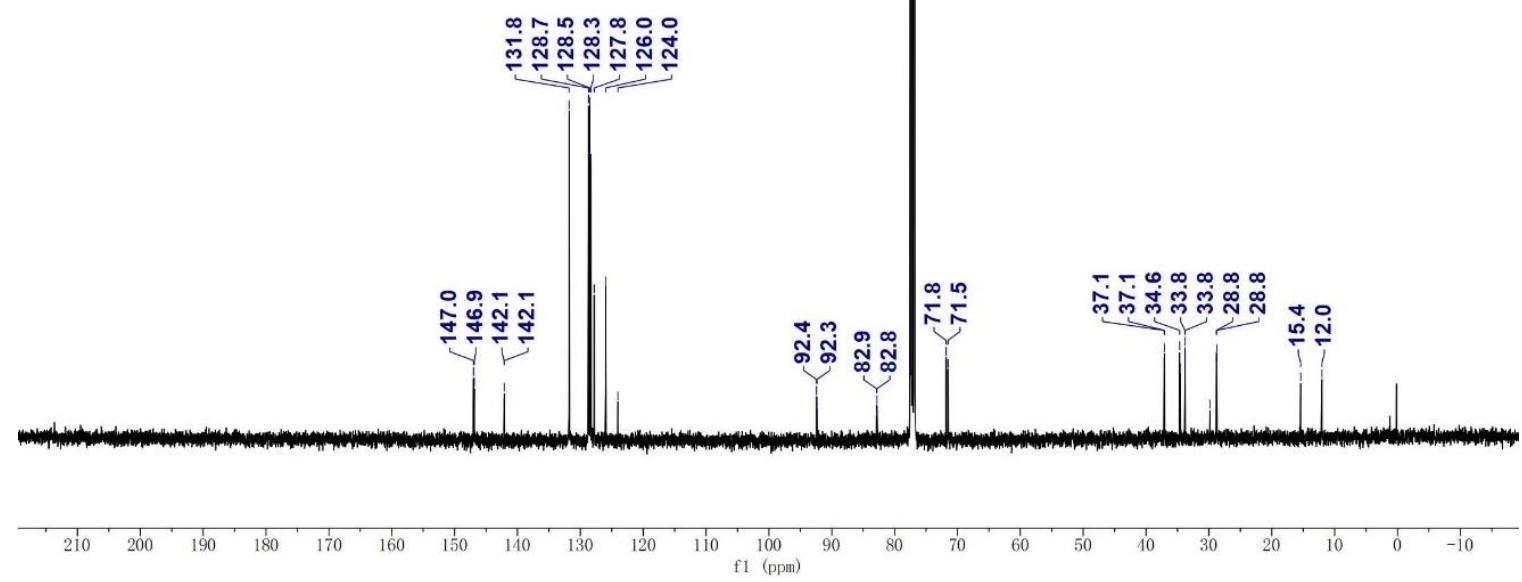

${ }^{13} \mathrm{C}$ NMR spectrum of $\mathbf{4 b}$ was measured in $\mathrm{CDCl}_{3}$ at $101 \mathrm{MHz}$ 

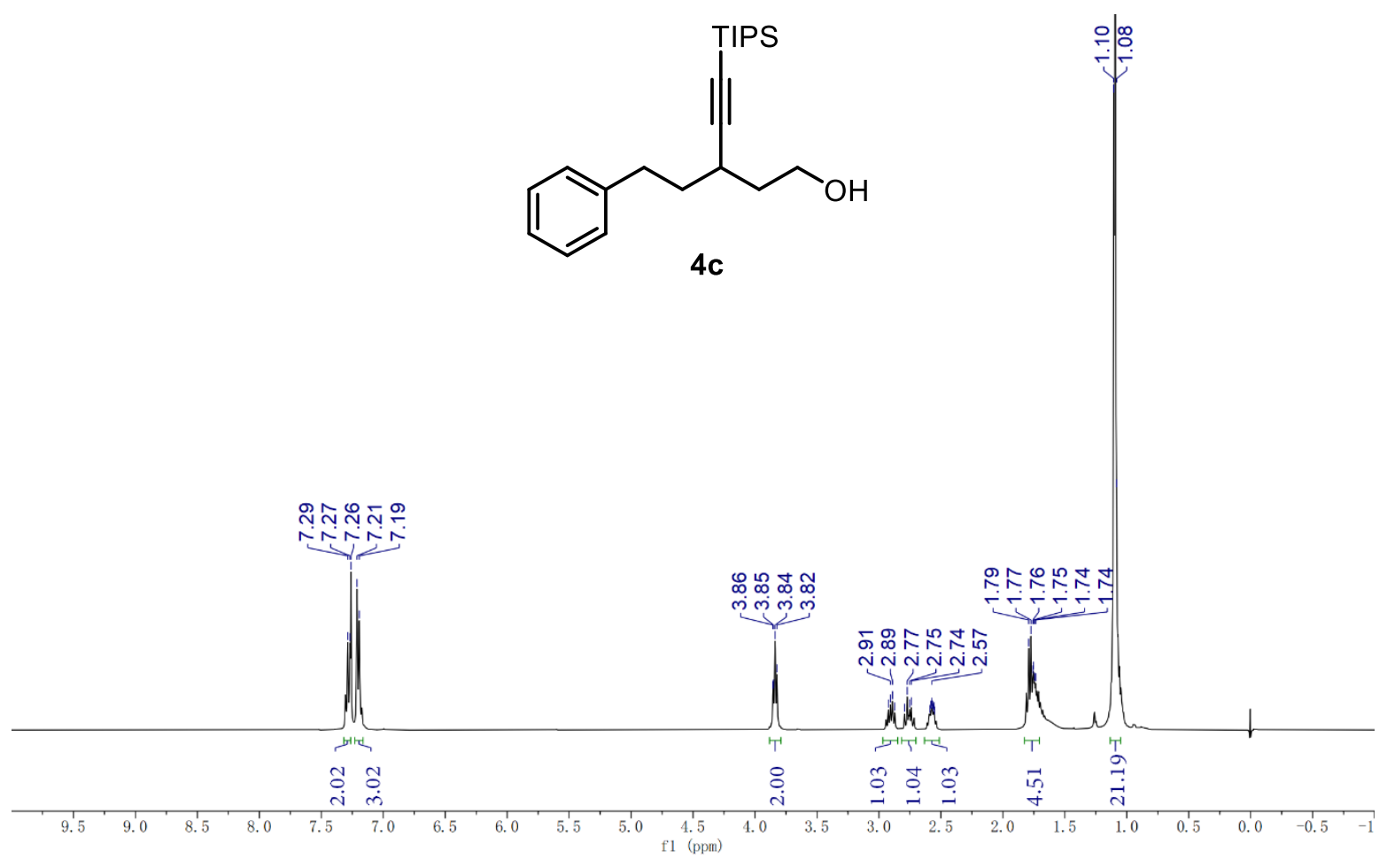

${ }^{1} \mathrm{H}$ NMR spectrum of $4 \mathrm{c}$ was measured in $\mathrm{CDCl}_{3}$ at $400 \mathrm{MH}$
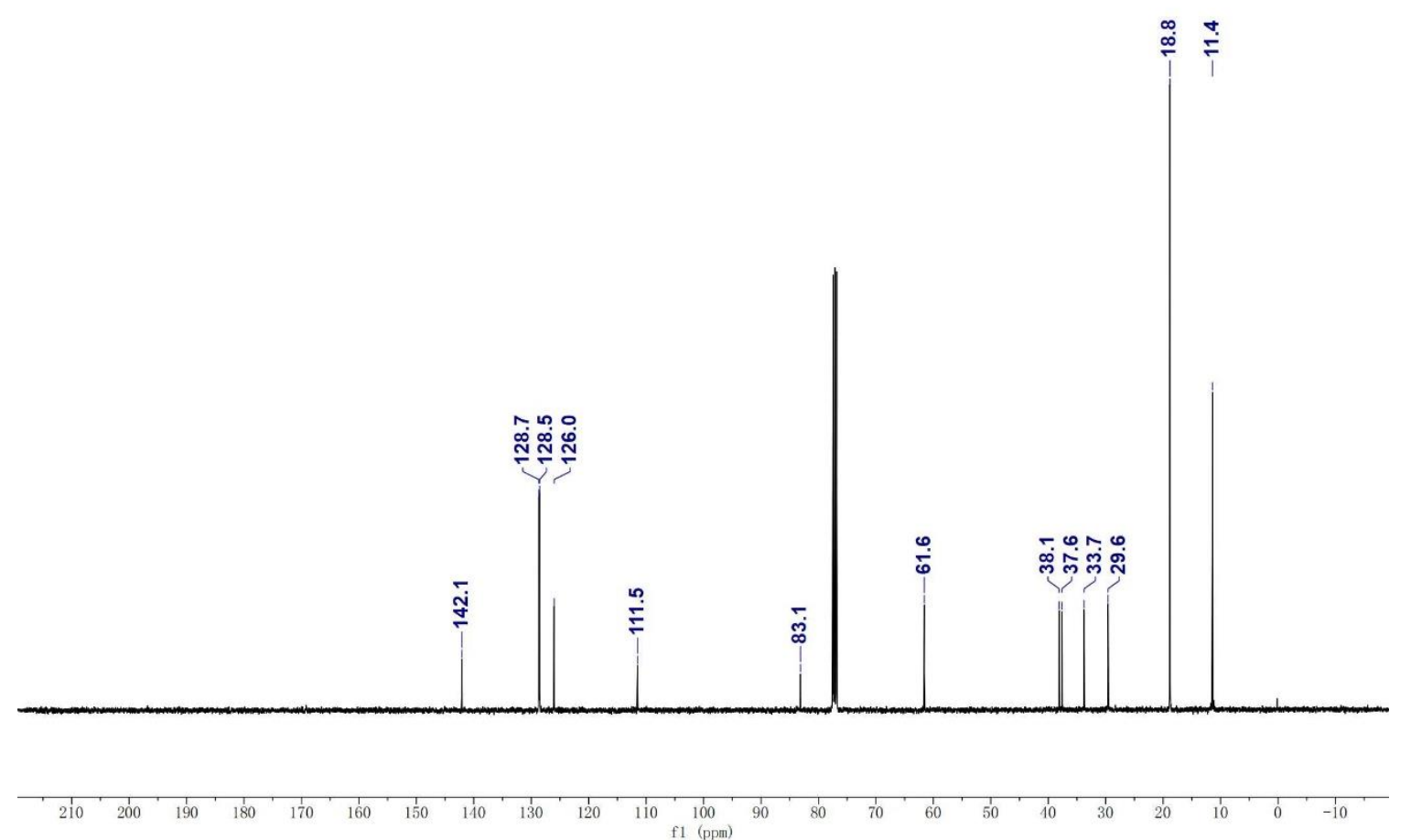

${ }^{13} \mathrm{C}$ NMR spectrum of $\mathbf{4 c}$ was measured in $\mathrm{CDCl}_{3}$ at $101 \mathrm{MHz}$ 